Portland State University

PDXScholar

Fall 12-12-2014

\title{
Strengths in Action: Implementing a Learning Organization Model in a Human Service Setting
}

\author{
Barbara Ann Whitbeck \\ Portland State University
}

Follow this and additional works at: https://pdxscholar.library.pdx.edu/open_access_etds

Part of the Organizational Behavior and Theory Commons, Social Work Commons, and the Sociology Commons

Let us know how access to this document benefits you.

\section{Recommended Citation}

Whitbeck, Barbara Ann, "Strengths in Action: Implementing a Learning Organization Model in a Human Service Setting" (2014). Dissertations and Theses. Paper 2095.

https://doi.org/10.15760/etd.2093

This Dissertation is brought to you for free and open access. It has been accepted for inclusion in Dissertations and Theses by an authorized administrator of PDXScholar. Please contact us if we can make this document more accessible: pdxscholar@pdx.edu. 
Strengths in Action: Implementing a Learning Organization Model

in a Human Service Setting

by

Barbara Ann Whitbeck

A dissertation submitted in partial fulfillment of the requirements for the degree of

Doctor of Philosophy

in

Social Work and Social Research

Dissertation Committee:

Maria Talbott, Chair

Eileen Brennan

Pauline Jivanjee

Karen Seccombe

Portland State University

2014 
(C) 2014 Barbara Ann Whitbeck 


\begin{abstract}
Although learning organization theory evolved in corporate settings, literature suggests that the theory has much to offer human service organizations. This dissertation examines the implementation of a modified learning organization model in three small field offices of a publicly-funded vocational rehabilitation organization in the Pacific Northwest, at a time when the organization was negotiating financial cutbacks and organizational changes. The model - known as Strengths in Action - was based on Senge's five learning organization disciplines, and informed by organizational culture theory. In each participating office, all staff worked together to set a goal, make a plan, and achieve the goal.

This dissertation covers the implementation of the modified learning organization model; the factors that facilitated and impeded the model's implementation; the model's impact on participating offices' climate and culture; and the similarities and differences among participating offices. This primarily qualitative study utilized mixed methods: observations, interviews, and an online survey.

Implementation of the model resulted in individual and team learning, better staff communication, more productive teamwork, stronger staff relationships, stronger office/community partner relationships, and improved office morale. This study shows that such a model can be effective in a human service setting, moving workgroups away from a mode of individual workers reactively handling individual cases, and toward a mode of proactive collective problem-solving. It also provides strong evidence that a learning organization model, implemented during a period of resource retrenchment, can
\end{abstract}


produce substantial benefits for small workgroups within human service organizations, even when the model is not disseminated organization-wide. 


\section{Dedication}

To

My late father, John Nicholas Arends, who gifted me with a love of learning and respect for those who "conclude well."

And to

My husband, Dr. John Kenneth Whitbeck, whose love, support and encouragement made my dissertation journey possible. 


\section{Acknowledgements}

\section{Chair}

Maria Talbott, $\mathrm{PhD}$ - advisor, professor, mentor, and friend - for always being there

\section{Committee Members}

- Eileen Brennan, $\mathrm{PhD}$ - for your consistent support and your thoughtful edits, which increased my knowledge and improved my work

- Pauline Jivanjee, $\mathrm{PhD}$ - for your warmth, your insights, and the opportunities you created for me throughout the $\mathrm{PhD}$ program

- Karen Seccombe, PhD (OGS representative) - for "taking me on" despite a myriad of professional and personal responsibilities, and for your keen and practical advice

\section{DVR Staff}

- DVR's director, management team, and all the Strengths in Action participants whose ideas and energy drove the Strengths in Action projects

\section{$\underline{\text { DSHS Staff }}$}

- Nancy Raiha, PhD, Barbara Felver, MES, MPA, Dario Longhi PhD, Jody BeckerGreen, PhD, and all the others who supported me during the SIA study

\section{Technical and Process Advisors}

- Dennis McBride, PhD, Kevin Ryser, Bob Drake, Pauline Duffy, Roxanne Treece, Thet Mar Win

\section{My Ever-Loyal Family}

- John, Mom, Patti, Tim Jason, Ryan, Meghan, Drew, Caleb, Molly, Lucien, Joel, Evan, Masayo and Ayana

\section{Friends, Colleagues and Mentors}

- Jim Blodgett, Theal Compton-Drake, Sandy Crouch, Sandy Desy, Warren Gohl, Christina Maleney, Cindy Marchand-Cecil, Lori McWilliams, Myrth Ogilvie, Dennis Olson, Mike and Nan Ponder, and George Uhlman. 


\section{Table of Contents}

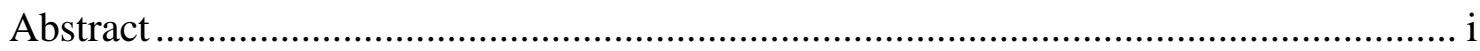

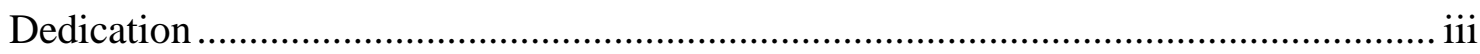

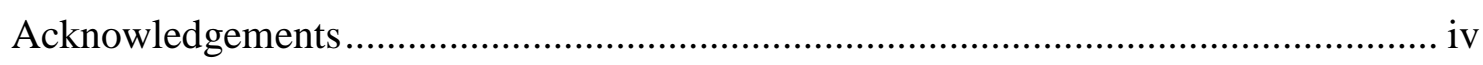

List of Tables ............................................................................................. vii

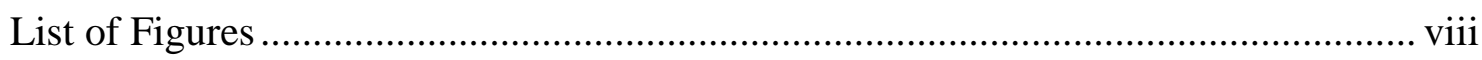

Chapter 1: Introduction and Overview ............................................................

Chapter 2: Literature Review ..........................................................................

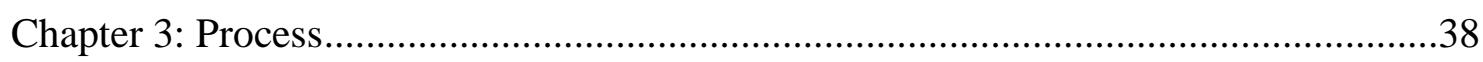

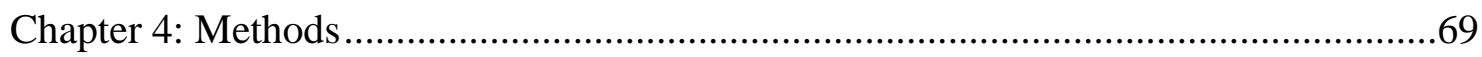

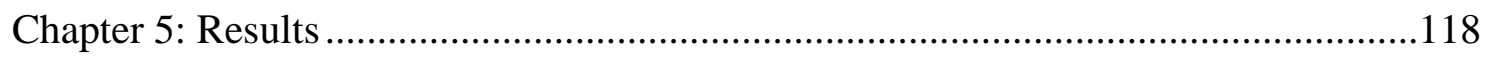

Chapter 6: Discussion and Conclusions...........................................................250

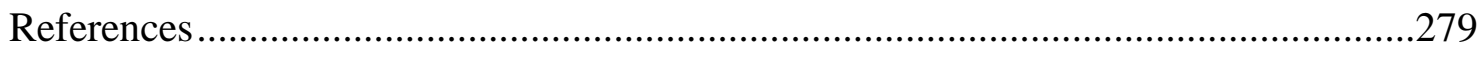

Appendix A — Learning from Offices Study Overview .......................................294

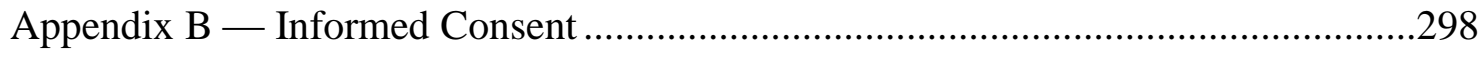

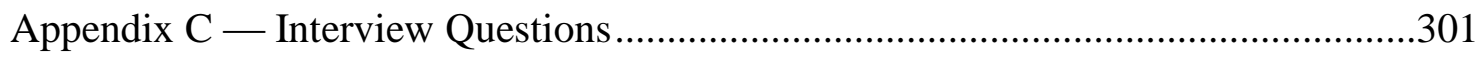

Appendix D — Online Survey ......................................................................

Appendix E — Description of Strengths in Action Study ......................................307

Appendix F — First Strengths in Action Agenda...................................................310 
Appendix G - Response Glossary

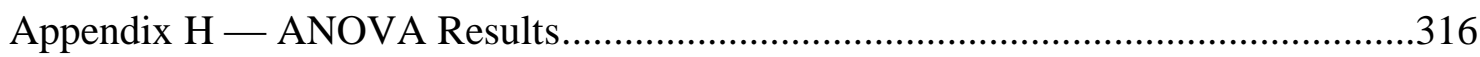

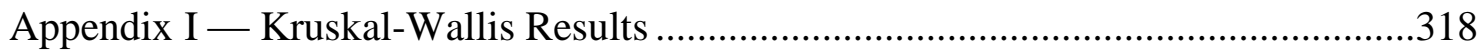

Appendix J — Online Survey Closed-ended Questions: Means, Standard Deviations, and Percentages of Positive Responses.......................................................................320 


\section{List of Tables}

Table 5.1 - Summary of Data Sources - Research Question 1................................... 138

Table 5.2 - Closed-ended survey questions - Personal Mastery ................................. 144

Table 5.3 - Closed-ended survey questions - Mental Models.................................... 149

Table 5.4 - Closed-ended survey questions- Building Shared Visions......................... 153

Table 5.5 - Closed-ended survey questions - Team Learning ................................... 157

Table 5.6 - Closed-ended survey questions - Systems Thinking ............................... 162

Table 5.7 - Summary of data sources - Research Question 2 ................................... 163

Table 5.8 - Closed-ended survey questions - Structure of SIA Project ....................... 172

Table 5.9 - Closed-ended survey questions - Supervisor Support .............................. 175

Table 5.10 - Closed-ended survey questions - Staff-to-Staff Connections.................... 180

Table 5.11 - Closed-ended survey questions - Staff/Community Partner Connections 185

Table 5.12 - Summary of data sources - Research Question 3 ................................. 190

Table 5.13 - Summary of data sources - Research Question 4 ................................. 211

Table 5.14 - Closed-ended survey questions - Supervisors' Approaches ..................... 218

Table 5.15 - Closed-ended survey questions - New Relationships among Staff ...........221

Table 5.16 - Closed-ended survey questions - New Community Partner Connections. 224

Table 5.17 - Summary of data sources - Research Question 5 ................................. 227 


\section{List of Figures}

Figure 4.1 - Diagram of interconnections among study methods ............................... 77

Figure 5.1 - Storyline for Research Question 1 ................................................. 138

Figure 5.2 - Outline of discussion of Research Question 1 Findings .......................... 140

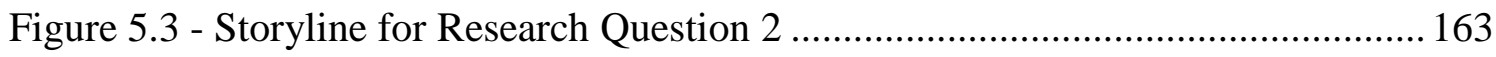

Figure 5.4 - Outline of discussion of Research Question 2 Findings .......................... 165

Figure 5.5 - Storyline for Research Question 3 ................................................... 190

Figure 5.6 - Outline of Discussion of Research Question 3 Findings ......................... 191

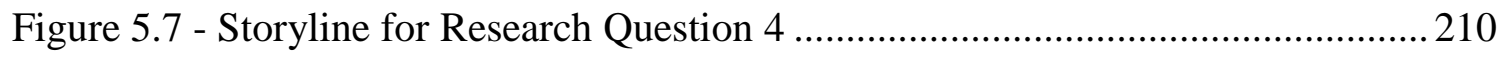

Figure 5.8 - Outline of Discussion of Research Question 4 Findings .........................212

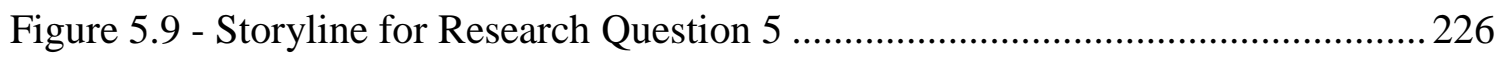

Figure 5.10 - Outline of discussion of Research Question 5 Findings ........................ 227 


\section{Chapter 1: Introduction and Overview}

\section{Research Context}

When the Strengths in Action (SIA) study was being developed in late 2008 and early 2009, the United States was in the midst of an economic crisis of historic proportions. The effects of this crisis were being felt by nearly every individual, organization, and governmental unit in the nation, and in economies throughout the world. The economic downturn - widely known as the Great Recession - officially ended in June 2009, the same month the SIA study began. However, the economic pain caused by the recession continued well beyond that point. More than five years later, many statistics that describe the U.S. economy have not returned to their pre-recession values (Goodman \& Mance, 2011; U.S. Department of Labor, Bureau of Labor Statistics, 2012, 2014a, 2014b).

During the recession and its aftermath, publicly-funded human service organizations, whose mission is to help some of the most vulnerable populations in our society, were faced with enormous challenges. As state revenues from income and sales taxes declined, state budgets for a wide variety of social services decreased. State-funded human service organizations responded by instituting hiring freezes, salary freezes, and cuts in employee benefits, and cancelling or severely restricting employee travel and training. In some states, including the state in which the SIA study was conducted, employees were asked to take furloughs or unpaid days off (Center for State \& Local Government Excellence, 2010, 2014; U.S. Department of Commerce, Economics and Statistics Administration, U.S. Census Bureau, 2014). 
State workers were not the only victims of the recession. Clients of state-funded human service organizations, whose numbers grew during rapidly during the recession, faced severe economic and emotional distress. While client needs escalated, many states made significant cuts in social service programs that provided access to vital services such as food, housing, and medical care (County Welfare Directors Association of California, \& California State Association of Counties, 2009; National Association of Social Workers, 2009; Rothstein \& Valletta, 2014; State of Washington Office of Financial Management, 2014 ; U.S. Government Accountability Office, 2012a, 2012b).

In short, during the SIA study, state revenues were down, the number of state workers was decreasing, and resources for clients of state-funded human service organizations were diminishing. All of this was happening at a time when the number and the needs of clients were increasing.

As of 2014, the nation's private economy is slowly but steadily improving, but similar progress has not been made in the public sector. State and local government employment has never returned to pre-2008 levels, which means that the workloads of many employees in publicly-funded human service organizations remain high (Center for State \& Local Government Excellence, 2014; U.S. Government Accountability Office, 2013). These heavy workloads, coupled with a chronic lack of needed resources, have a negative impact on employee morale and the quality of client service (Arrington, P., 2008; Whitaker, Weismiller, \& Clark, 2006). 


\section{Research Problem}

Particularly in climates fraught with economic constraints and ongoing changes, the humans working in human service organizations are the organizations' most important assets (Austin \& Hopkins, 2004; Lewis, Lewis, Packard, \& Souflee, 2001; Schorr, 1997). The relationships that staff members establish with coworkers, clients, and community partners are vital to organizations' current effectiveness and future growth (Austin \& Hopkins, 2004; Leana \& Rousseau, 2000; Pil \& Leana, 2000; Van Buren \& Leana, 2000). Because of this, managers and supervisors need to pay close attention to the climate and morale in their organization. They need to take actions that make staff feel useful and appreciated, and encourage staff to use their minds and skills to craft creative, and cost-effective, solutions to client problems (Austin, 2008; Beddoe, 2009; Brody, 2005; Glisson, 2000; Lewis et al., 2001; Maden, 2011; Maynard, 2010; Schein, 1992; Senge, 1990; Senge and Kaufer, 2000).

However, there is a problem. Without permission, encouragement, support and resources from an organization's leaders, supervisors and managers in human service organizations have little incentive to take the time or develop the skills necessary to empower staff. Their incentive is further reduced when they are struggling to deal with economic constraints, workload pressures, and organizational changes on a daily basis. How can this problem be addressed?

Literature suggests that learning organization theory has much to offer human service organizations negotiating financial difficulties and organizational changes, and that leaders of such organizations would be wise to promote learning organization efforts. 
In a functioning learning organization, regardless of current circumstances, supervisors and managers model positive and proactive behaviors for their staff; teams work together to generate creative ideas, and act upon them; and there is an organization-wide focus on and commitment to staff empowerment, motivation, and morale. In turn, staff model the support and encouragement they have received, and share the skills and knowledge they have developed, in their interactions with clients and community partners, resulting in more effective services for clients (Austin, 2008; Bandura, 1997; Bargal, 2000; Beddoe, 2009; Brody, 2005; Gustavsson, 2009; Iglehart, 2000; Leana \& Rousseau, 2000; Lewis et al., 2001; Lindberg \& Meredith, 2012; Maden, 2011; Maynard, 2010; Pil and Leana, 2000; Senge 2006; Senge et. al, 1999; Schein, 1992; Schorr, 1997; Van Buren \& Leana, 2000).

\section{Research Purpose and Dissertation Description}

The purpose of the SIA study was to examine the implementation of a learning organization model in a human service setting. This dissertation documents the development and implementation of SIA, a modified learning organization model. The development of the SIA model began in the fall of 2008. The implementation of the SIA model took place in three field offices in a state vocational rehabilitation (VR) organization in the Pacific Northwest between June 2009 and May 2010.

The SIA study data were drawn from researcher observations, participant interviews, and an online survey. The analyses of qualitative and quantitative data collected focused on specific factors that facilitated or impeded the model's implementation, the effect the implementation of the model had on the participating 
offices' climate and culture, and whether the experiences and outcomes in the three offices differed. More broadly, the analyses examined the extent to which the implementation of the model energized and empowered staff, and encouraged them to work collaboratively to provide the best possible service to clients in a period of economic difficulties and organizational change.

\section{Research Questions}

The SIA study's research questions were:

1. To what extent was the modified learning organization model implemented?

2. What factors facilitated the adoption of the modified learning organization model?

3. What factors impeded the adoption of the modified learning organization model?

4. What impact did the adoption (or partial adoption) of a modified learning organization model have on office climate and culture?

5. In regard to the previous questions, what differences, if any, were found among the three VR offices participating in the study?

\section{Roadmap for Readers}

This chapter, Introduction and Overview, is the first of the dissertation's six chapters. Chapter 2, Literature Review, provides an overview of learning organization theory, the relationship of organizational culture theory to learning organization theory, and the implementation of learning organization models in human service settings. Chapter 3, Process, describes how the SIA study evolved, including circumstances and events that predated and informed the study, and the approach taken to making decisions and accomplishing tasks throughout the study. Chapter 4, Methods, outlines the methods 
used in the study, including the study's design, the research questions, and the qualitative and quantitative methods of data collection and analysis that were employed. Chapter 5, Results, which begins with a description of the three offices participating in the study, contains the results of data analysis for each of the five research questions. Chapter 6 , Discussion and Conclusions, outlines the key findings, examines the practice and policy implications of these findings for human service organizations, addresses my role as researcher, discusses research limitations and directions for future research, and summarizes the research conclusions. Following Chapter 6, references and appendices referred to in the body of the dissertation are provided. 


\section{Chapter 2: Literature Review}

Learning organization theory guided and framed the development, implementation and analysis of the SIA study. Organizational culture theory also informed SIA from its genesis to its completion.

The majority of this chapter reviews literature pertaining to learning organization theory and its application in human service arenas. The chapter begins with a brief overview of organizational open systems theories, the larger group of theories to which learning organization belongs. It then provides a synopsis of literature on learning organization theory - its evolution, its five disciplines, its strengths, and its limitations.

This chapter also addresses organizational culture theory, and its relationship to learning organization theory; learning organization theory's roots in the business world; and the implementation of learning organization models in human service settings.

\section{Organizational Open Systems Theories}

Prior to the 1970 s, organizational theorists tended to view each organization as a world unto itself. They recognized each organization as a system, acknowledging that a change in any element within that system caused changes in other elements within that system, but paid little attention to the effect of external factors on the organization, or the organization's effect on its external environment (Lawrence \& Lorsch, 2001; Lewis, et al., 2001; Thompson, 1992).

In contrast, the open systems theories that emerged in the 1970s (which evolved from general systems theory, proposed by biologist Ludwig von Bertalanffy in 1928), recognize that an organization's possibilities are determined both by internal factors such 
as management style, employees' characteristics, or production processes, and by external factors, such as the local economy, or working relationships within the community. They see that different units within an organization are affected in various and significant ways by their interactions with the environment outside the organization. They also recognize that the choices made inside an organization affect the environment (e.g., clients, other organizations, community) around it (Katz \& Kahn, 1992; Lewis, et al., 2001; Walonick, 1993).

In social sciences, an open system is a process that allows a system to exchange material, energy, people, capital, and information with its environment. In Alfred Kuhn's (1974) social systems model, communication (the exchange of information) and transaction (the exchanges of matter-energy) are the methods by which a system achieves equilibrium; all organizational and social interactions involve one or both of these methods.

Over the last four decades, a wide variety of practice models incorporating open systems concepts have developed. Some limit their focus to a particular type of organization, such as professional or governmental bureaucracies. Others promote a particular style of management, such as Japanese management (flexible job descriptions, and collective decision-making and responsibility) or Quality of Work Life (line worker involvement in organization decisions, and respect, social support, and challenging work for all). All value inter-agency teamwork and non-authoritarian management styles. (Lewis, et al., 2001; McKenna, 1994). 
Open systems theories advocate that managers and their employees see their organization as an ongoing process rather than as a static structure, and constantly assess current circumstances and needed changes. In addition, they must understand that the goals and activities they choose as their organizations' focus will inevitably be influenced by environmental factors beyond their control (Katz \& Kahn, 1992). This perspective fits well with human service organizations, in which environmental effects on individual clients and on the program as a whole must be constantly taken into consideration. It encourages human service professionals to see themselves as part of a larger network that, as a totality, can serve the individual client in a coordinated way (Austin \& Hopkins, 2004; Brody, 2005).

Of all the open systems theories, learning organization theory, with its emphasis on the development of both individual and shared visions, personal and team learning, and continuous review of and reflection upon organizational processes and goals, appears to have the most power to help publicly-funded human service organizations adapt to the continuous and complex changes they must negotiate.

\section{Learning Organization Theory}

The seeds of learning organization theory were planted in a wide variety of academic and organizational environments over the course of several decades. One of the first and most influential theorists in the arena of learning systems and reflective practice within organizations was Donald Schon. In Beyond the Stable State, Schon (1973) posited that there can no longer be an expectation that institutions will retain any 
particular form for any length of time, so it is critical that we "learn to understand, guide, influence, and manage these transformations" (p. 28).

Learning organization theory first gained major recognition in 1990 with the publication of Peter Senge's The Fifth Discipline: The Art and Practice of the Learning Organization (Chawla \& Renesch, 1995). According to Senge (1990):

Learning organizations are organizations where people continually expand their capacity to create the results they truly desire, where new and expansive patterns of thinking are nurtured, where collective aspiration is set free, and where people are continually learning how to learn together...The organizations that will truly excel in the future will be organizations that discover how to tap people's commitment and capacity to learn at all levels in an organization (pp. 3-4).

Senge $(1990,2001)$ outlined five disciplines - personal mastery, mental models, building shared visions, team learning, and systems thinking - that, together, form the basis of learning organization theory. He described a discipline as a body of theory and technique that must be studied and mastered to be put into practice, and as an ongoing, even lifelong, activity.

Senge underscored that practicing disciplines is different than emulating models. In his view, simply following best practices can do more harm than good, often leading to incomplete implementation and disappointing outcomes. In his words, "I do not believe great organizations have ever been built by trying to emulate another, any more than individual greatness is achieved by trying to copy another 'great person"' (Senge, 2001, p. 471). Here, he is not denying the value of learning from other organizations, or from 
well-conducted research. Rather, he is suggesting the importance of understanding and accommodating the nuances of each organization, and recognizing and responding to inevitable changes in organizations' environment as they occur.

\section{The five disciplines.}

\section{Personal mastery.}

Personal mastery is taking the time, and intellectual and emotional effort, to clarify, and then deepen, one's personal vision. In essence, it is an individual figuring out what he or she considers truly important (Senge, 1990; Senge, Kleiner, Roberts, Ross, Roth, \& Smith, 1999).

The central principle of personal mastery is creative tension. This involves using the gap between one's personal vision and one's current reality to generate energy for change. In order to do this effectively, individuals must have a clear view of both their vision and their reality (Senge, 1990, 2001).

Individuals striving for personal mastery are committed to life-long learning, and ever-increasing proficiency in their work, which they approach almost as a work of art. They are particularly interested in understanding how their actions affect others around them. Their goal is to attain expertise which can be shared with others, not to dominate others. They focus their energies on desired results; they are much less concerned with the particular means or processes used to attain those results, which leaves their mind open to innovate and create solutions to problems (Senge, 1990, 2001). 
Personal learning and organizational learning are reciprocal, and much good can come from the connections between the two. Individuals can gain much, including insights, new skills, and new behaviors, from participating in an organizational learning process. At the same time, an organization's commitment to and capacity for learning can be no greater than that of its members (Senge, 2001; Senge, Roberts, Ross, Smith \& Kleiner, 1994).

\section{Mental models.}

Mental models are deeply ingrained assumptions/generalizations that influence how one understands the world, behaves in the world, and believes the world works. They are the lenses through which individuals view their world (Senge, 1990).

In order to examine mental models, one must first "turn the mirror inward" (Senge, 2001, p. 470) to unearth one's internal pictures of the world, and then bring those pictures to the surface and scrutinize them. Once an individual has accomplished this process, he or she must share his or her own thinking with others and expose it to their influence, so limitations in his or her worldview can be recognized (Senge, 2001).

\section{Building shared visions.}

Building shared visions is the process of translating individual visions into a shared picture of the future that an organization, or a workgroup within an organization, wants to create. A shared vision is much more than the vision statements that have been so popular in U. S. organizations over the past few decades, which are often the vision of a single person or small group imposed on the rest of an organization. A true shared vision is a common caring about a goal shared by all members of a working group, and 
provides the focus and energy for subsequent team learning (Senge, 1990; Senge, et al., 1999).

There is a basic and important distinction between positive and negative visions. Asking the question, "What do we want?" (i.e., aspiring to something) is totally different than asking the question, "What do we want to avoid?" (i.e., being afraid of something). While fear can produce notable results in the short term, aspiration endures as a source of learning and growth (Marquardt, 2002; Senge, 1990).

The only way to arrive at a genuinely shared vision is to give each person with a stake in the organization's (or workgroup's) future a legitimate voice in the process. One cannot dictate a vision; it can only emerge if all parties have bought in to the process and have a long-term commitment to it (Senge, 1990).

Team learning.

Team learning is a process in which coworkers in an organization "think together" until there is consensus about how to reach a particular goal. Team learning is vital because teams, not individuals, are the fundamental learning unit in modern organizations. Unless teams in an organization can learn, the organization cannot learn (Reason \& Bradbury, 2001; Senge, 1990).

Team learning starts with dialogue. Three basic conditions must be present for dialogue to occur. First, team members must suspend their assumptions. This means they must be cognizant of their assumptions, and willing to hold them for personal and group examination. Second, they must regard one another as colleagues, and give up any privileges of rank they normally are granted. Third, they must be guided, at least in the 
early stages of the process, by a facilitator. The facilitator's responsibilities include helping team members maintain ownership of the process and outcomes, keeping the dialogue moving, and reminding others of the conditions of dialogue (Senge, 1990).

Effective team learning balances dialogue and discussion. In dialogue, complex issues are explored, and no agreement is sought. In discussion, different views are presented and defended, and decisions are made. Both dialogue and discussion can lead to new behaviors and actions. However, these behaviors and actions are simply a byproduct of dialogue, while they are often the focus of discussion (Senge, 1990; Senge, et al., 1999).

Team members who enter into dialogue regularly tend to develop a deep trust, and an understanding and respect for one another's perspectives, that carry over into discussions. Good teams often are in conflict; the free flow of conflicting ideas is essential for creative thinking. Negative patterns of interaction, such as defensiveness, can undermine dialogue, but if such patterns are recognized and addressed, they can actually accelerate learning (Senge, 1990; Bennett \& Brown, 1995).

When teams are truly learning, they can produce extraordinary results. Groups can see the larger picture and arrive at insights that could not be attained individually; individual team members can grow more rapidly than they could on their own (Senge, 1990; Senge, et al., 1994; Senge, et. al, 1999).

\section{Systems thinking.}

Systems thinking is looking at the interrelated parts of a process, and recognizing and examining patterns and possibilities. In the context of learning organization theory, 
Senge (1990) refers to systems thinking as the fifth discipline. It is the discipline that integrates the other four disciplines - personal mastery, mental models, building shared visions, and team learning - into a coherent body of theory and practice.

Organizations are systems, comprised of interrelated parts and actions which often take years to fully play out their effects on each other. If you are inside an organizational system (e.g., an employee) it is hard to see or intuit full patterns of interactions and change; you can only see snapshots. When you make decisions based on such limited information, it is hard to make good choices that solve underlying problems (Senge, 1990; Kofman \& Senge, 1995).

In Senge's (1990) view, if all five disciplines develop independently, much of the potential value of each discipline will be lost. If, on the other hand, all five disciplines develop as an ensemble, and if an organization's employees consistently look at how each of the disciplines is connected to the others, the whole (the outcomes) can far exceed the sum of its parts (the inputs).

\section{Strengths of learning organization theory.}

In this section of the report, six strengths of learning organization theory are examined, with special emphasis on their value in human service settings.

\section{Guidelines for supervisors and managers.}

One strength of learning organization theory is that it establishes clear guidelines for supervisors and managers operating within learning organizations. Three key functions outlined for both supervisors and managers: researcher, designer and protector of learning processes, and role model (Senge, 1990; Senge \& Kaufer, 2000). 
Being a researcher means supervisors and managers must understand both the internal and external trends and forces that are driving change in their organization, notice changes in the environment, and figure out what changes the organization needs to make to remain adaptive (Senge, 1990; Schein, 1992). For human service organizations to function well, a keen awareness of developments outside the organization is particularly important. As Farmbry (2004) points out:

Communities in which human service agencies work are changing on a rapid and regular basis... with the growth in populations of color, new immigrant groups, the elderly, and groups with disabilities, the dimensions of community complexity grow... organizations need to be able to comprehend such complexities. Supervisors have a role in ensuring that such understanding occurs within an organization (p. 254).

Being a designer and protector of learning processes means supervisors and managers must introduce, promote, and protect processes their staff can use to deal productively with the critical issues they face (Senge, 1990; Senge et al., 1999). This is vital in human service organizations because any process that involves staff, "will be more likely to result in a design that will respond effectively to client needs and provide a high quality of working life for staff" (Lewis, et al., 2001).

Last but not least, supervisors and managers in learning organizations need to be role models for their staff. According to Senge (1990), they should lead with actions rather than words, and should refrain from pushing their ideas onto others, because 
"people learn what they need to learn, not what someone else thinks they need to learn" (p. 345).

\section{Individual learning and growth.}

A second strength of learning organization theory is that it provides opportunities for individuals to learn and grow. Organizations committed to a learning organization model actively encourage their staff to take time to define and explore their values, to clarify for themselves what they have to contribute to the organization, and to expand their individual learning and capabilities in the context of group learning (Senge, 1990; Senge et al., 1999).

Employees typically seek more than purely economic rewards from their employment. Particularly in human service settings, work is an important source of employees' selfimage and self-worth, and a means of self-actualization (Brody, 2005; Edwards \& Wajcman, 2005; Lewis et al., 2001). Studies have shown that people want the opportunity to do meaningful work that has a clear purpose; accomplishes something they believe contributes to achieving an important goal; and permits them to realize their full potential (Ashmos \& Duchon, 2000; Mitroff \& Denton, 1999). Organizations have discovered that giving individuals learning opportunities that lead to expanded work options improves both employee satisfaction and employee retention (Austin \& Hopkins, 2004; Buckingham \& Coffman, 1999; Kaye \& Jordan-Evans, 2005).

Bandura's (1997) research on self-efficacy underscores the importance of providing employees with opportunities to advance their knowledge and skills. He found 
that people who come to believe they are capable of organizing and executing the steps necessary to attain a goal (at work, and in other settings) are much more likely to pursue that goal and, conversely, "If people believe they have no power to produce results, they will not attempt to make things happen" (p. 3).

\section{Organizational learning and growth.}

A third strength of learning organization theory is that it creates opportunities for organizations as a whole to learn and grow. It does this by encouraging employees to share their visions for the organization, learn together, and think systematically (Senge, 1990; Senge et al., 1994). Organizations that promote communication and collaboration among employees at all levels, and encourage employees to respond to problems and changes by asking one another questions such as "What can we learn from this?" and "What can we do better?," increase their potential to "learn their way out of their problems" (Austin \& Hopkins, 2004, p. 4).

When organizations actively encourage employees' input and collaboration, it increases organizational social capital, which is defined as the nature of social relations within an organization, and how effectively the employees of an organization work as a team (Pil \& Leana, 2000; Van Buren \& Leana, 2000). Social capital, sometimes called relational wealth, is one of the strongest assets of any organization (Leana \& Rousseau, 2000). It is particularly vital in human service settings, where fixed assets such as capital, buildings and equipment are often in short supply (Austin \& Hopkins, 2004; Brody, 2005). 
A work environment where staff have strong relationships and work together well helps individual employees justify their commitment to the collective good; provides a base from which work can be flexibly organized; reduces the need for formal contracts, incentives, and monitoring mechanisms; and, as employees are well aware of "who knows what" within the organization, assures that individual expertise is used to advantage (Leana \& Rousseau, 2000; Van Buren \& Leana, 2000). An organizational culture where open and honest communication is the norm, staff come to know one another's strengths and interests, and staff work together in pursuit of common goals, has the twin advantages of empowering staff (Iglehart, 2000; Lewis, et al., 2001; McKenna, 1994) and building cultural competence (Fong \& Furuto, 2001; Schneider \& Smith, 2004; Solomon, 2001).

\section{Learning and growth in workgroups.}

A fourth strength of learning organization theory is that it can benefit small workgroups within an organization. Particularly in large human service organizations, learning and growth do not always occur evenly or consistently throughout the organization (Senge \& Kaufer, 2000). Frequently, new ideas and innovative activities first occur within pockets of an organization. According to Austin and Hopkins (2004):

When employees start sharing information, ideas, and knowledge gained, they create a learning culture through the sharing, even if the organization has not yet developed a fully collaborative culture... Research shows that is it more common to see learning and subsequent improvement or change taking place in individuals 
or small groups or teams than as a coordinated effort across an organization (pp. 4-5).

Organizational learning, then, is usually not a single process performed by the entire organization in a uniform fashion, but rather various organizational units engaging in different forms of learning at different levels of intensity (Lipshitz \& Popper, 2000). Ideally, innovations that are born in sub-units of an organization are eventually disseminated throughout the organization. However, even if that fails to occur, individual workgroups can reap real and long-lasting benefits from the changes they create (Baldwin, 2008, Chowdhury, 2000; Schein, 1992, Senge \& Kaufer, 2000).

Team projects increase employee satisfaction and employee commitment, both because they foster a sense of connection between coworkers (Brody, 2005; Pfeffer, 2006; Schneider \& Smith, 2004) and because people want to be part of something larger than themselves, to help create something important (Senge, 1990). Speaking specifically of human service organizations, Brody (2005) offers:

Because those who are actively involved in decision making are most likely to have the enthusiasm for implementing those decisions, agency managers should seek meaningful ways to foster staff participation. Forming staff into teams is one of the most effective methods of developing staff commitment (p. 384).

\section{Connections with clients and community partners.}

A fifth strength of learning organization theory is its potential to promote strong connections between organizations and their clients and community partners. This is 
critical for human service organizations, whose mission is to serve their clients, and who cannot do their best work without the support of the larger community.

Learning organization theorists have been criticized for focusing almost exclusively on relationships and activities within organizations, and failing to address organizations' external relationships (Poell, 2005). However, there is growing evidence that if a human service organization - or sub-units within such an organization - embraces a learning organization model, it is very likely to have a positive impact on that organization's relationships with individuals and groups in the community (Austin \& Hopkins, 2004; Lewis et al., 2001).

In human service organizations, the methods used by supervisors and managers to address the concerns of workers are similar to those used by workers to address client problems and needs; the two are, in essence, a parallel process (Ganzer \& Ornstein, 1999; Guitierrez, GlenMaye, \& Delois, 1995; Ladany, Constantine, Mileer, Erickson, \& MuseBurke, 2000; Shulman, 1993). If employees feel supported within a learning culture, they tend to relate to clients in an open and supportive manner, are better able to help clients develop solutions to their own problems, and thereby empower clients to set and attain personal goals (Austin \& Hopkins, 2004; Bargal, 2000). Conversely, if employees experience their work environment as non-supportive, impersonal, and stressful, their interactions with clients will reflect the negativity of the environment (Schneider, White, \& Paul, 1998; Glisson, 2000).

Effective supervisors and managers within learning organizations are skilled at building, and supporting, teams that learn and work well together. These leaders can, and 
often do, use their team-building skills to forge productive relationships with key individuals and agencies in their organization's community; the best among them encourage their staff to do the same (Austin \& Hopkins, 2004; Brody, 2005, Schorr, 1997).

The concept of organizational social capital, discussed earlier in regard to relationships within organizations, also encompasses the ability of an organization to work effectively with clients, other organizations, and the community as a whole. Employees of human services organizations who build strong working relationships with clients and community partners are creating a genuine form of wealth for their organization and those it serves (Leana \& Rousseau, 2000).

\section{Ability to adapt to change.}

A sixth strength of learning organization theory, which draws on each of the five strengths outlined above, is its ability to help organizations adapt to inevitable internal and external changes. This is important because, as Schein (1992) states so succinctly, "We basically don't know what the world of tomorrow will really be like, except that it will be different. That means that organizations and their leaders will have to become perpetual learners" (p. 361). However, to emphasize how important it is for organizations to be able to adapt to changes in circumstance is not to discount the importance of organizational stability. "Learning systems need to maintain their identity, and...the self-identity of those who belong to them, but they must at the same time be capable of transforming themselves" (Schon, 1973, p. 57). 
Learning organizations can strike a balance between stability and flexibility by keeping their focus on clearly defined outcomes, and allowing a good deal of flexibility in the processes that lead to those desired outcomes (Senge, 1990, 2001). According to Schorr (1997):

Successful programs have a clear mission, and continue to evolve over time. They create an organizational culture that is outcome oriented rather than rulebound. They combine a highly flexible mode of operation with a clear sense of mission, which everyone associated with the organization can articulate in simple terms. They are "tight" about their mission and simultaneously "loose" about how it is carried out. (p. 8).

One distinguishing feature of learning organizations is the level of trust they grant their employees. This is reflected in the amount of information management provides line staff regarding changes the organization is facing, and the encouragement staff is given to generate and act upon innovative responses to those changes. Using the collective wisdom of all staff spawns, grows, and sustains creative processes (Senge, 1990; Senge et al., 1994). In human service organizations this means, "front-line staff are able to exercise enough discretion so they can help people get what they need when they need it...not everything to everybody...but more than a single strand of support" (Schorr, 1997, p. 5).

In sum, in order to adapt effectively to significant and ongoing changes in clients' needs, human service organizations will need to encourage and make good use of the commitment and creativity of their all their staff. As Schein (1992) observes: 
Learning and change cannot be imposed on people. Their involvement and participation are needed diagnosing what is going on, figuring out what to do, and actually doing it. The more turbulent, ambiguous, and out of control the world becomes, the more the learning process will have to be shared by all the members of the social unit doing the learning. If the leaders of today want organizational cultures that will themselves be more amenable to learning they will have to set the example by becoming learners themselves and involving others in the learning process (p. 392).

\section{Limitations of learning organization theory.}

Learning organization theory is not without its critics, and not without issues related to its implementation in organizational settings. In this section of the report, the major criticism of learning organization theory is presented. In addition, six potential issues related to implementing the theory are outlined, with particular attention paid to how they may play out in human service settings.

\section{Learning organization theory ignores power differentials.}

The primary criticism of learning organization theory is that it fails to take into account the issues of power, domination, and compliance that are a fact of organizational life. Critics point out that granting power to workers in specific situations in no way equalizes power, as it fails to factor self-imposed controls, peer controls within selfmanaged teams, and organization-wide indoctrination and culture (Beddoe, 2009; Caldwell, 2011). Fenwick (1998) contends the learning organization discourse, "presents itself as a romantic ideal encouraging workers' personal growth and imaginative 
engagement - yet this discourse continues the workplace tradition of dictating which kind of growth counts most" (p. 152).

\section{Implementation can be complicated and time-consuming.}

One issue, particularly in large organizations, is that implementation of learning organization models can be difficult and time-consuming. In the words of Lewis, et al. (2001), it is "very complicated stuff" (p. 319). In human service organizations, managers are often loathe to burden staff already overwhelmed by tasks and deadlines with yet another responsibility. Also, innovations that evolve and work well in pockets of an organization are not always quickly or easily diffused to the larger organization (Davidson \& McMahon, 1999; Baldwin, 2008). However, once a learning culture is established in a human service organization, it can pay back an organization in a myriad of ways over an extended period of time (Austin \& Hopkins, 2004; Herman, 2007; Menefee, 2004).

\section{Innovations may break organizational rules.}

A second issue is that innovative ideas generated in team learning processes can run counter to existing organizational rules and regulations (Davidson \& McMahon, 1999). This is pertinent to human service organizations, many of whom operate under stringent state and federal statutes and guidelines. However, organizations committed to the implementation of a learning organization model can encourage employees to craft processes that will work within existing constraints, and managers can choose to petition legislatures and other authorizing entities for clearly needed changes to existing statutes or policies. 


\section{Supervisors and managers may fear giving staff voice.}

A third issue that arises in the implementation of learning organization models is that managers and supervisors tend to be nervous and threatened about inviting employee participation in organizational decision-making, fearing they will be unable to direct or control the energy that is released (Senge, 1990). In regard to human service managers, Austin and Hopkins (2004) offer, "There seems to be a fear that involving employees means losing authority. Supervisors and administrators need to remember that staff have a voice, not a veto" (p. 309). A number of studies have shown that staff involvement in decision-making did not undermine the authority of organizational leaders, and clearly helped clients, staff, and the organization as a whole (Annie E. Casey Foundation Casey Family Services, n.d.; Giesecke \& McNeil, 2004; Gustavsson, 2009; Ruffolo, Kuhn, et al., 2006).

\section{Staff may choose not to participate in decision-making processes.}

The failure of some staff to participate, or participate fully, in decision-making processes is a fourth issue when implementing learning organization models. Just as managers and supervisors may be unenthusiastic about involving employees in organizational decision-making, employees who are distrustful, disengaged, or overextended may be actively or passively resistant to taking part in decision-making processes (Lindberg \& Meredith, 2012; Ruffolo, Kuhn, \& Evans, 2006). There is evidence that workers' participation levels can be altered over time, but only if supervisors and managers send a clear message that their input is genuinely valued, and 
actively encourage their involvement on an ongoing basis (Annie E. Casey Foundation Casey Family Services, n.d.; Giesecke \& McNeil, 2004; Herman, 2007; Maden, 2011).

\section{Communications in group settings may be less than straightforward.}

A fifth issue is difficulty facilitating open and honest communication in learning organization processes. Human service organizations value relationships and diversity; the tone is one of accommodation. A strong learning culture requires exchanging and debating ideas within the context of team learning; the tone can at times be one of challenge and dissent. Unless managers and supervisors explicitly encourage and support employees in taking the risk to challenge the organization's status quo, employees will not feel safe in doing so, and the benefits of a learning culture will not be realized (Austin \& Hopkins, 2004; Senge, 1990).

\section{Implementation may be "shut down" when it is needed most.}

A sixth issue is that organizations tend to revert to a more hierarchical structure in times of crisis. Faced with uncertainty, their first reaction is most often to impose control from the top down. This frequently involves dispensing with innovative ground-level programs and services just at the time they are most needed, and sacrificing lower-level decision-making, which is a critical adaptive tool when change is occurring rapidly. It is therefore not surprising that workgroup-level learning organization efforts, which are innovative and promote lower-level decision-making, are often abandoned by organizations going through financial difficulties or significant internal changes (Baldwin, 2008; Caldwell, 2011).

\section{Organizational Culture Theory}


Organizational culture theorists believe that individual organizations, like larger societies, have a distinct culture, comprised of the organization's values, beliefs, assumptions, perceptions, behavioral norms, artifacts, and patterns of behavior (Shafritz \& Ott, 1992). Organizational culture can be strong or weak, and have positive or negative effects (or both) on staff and the workplace. In many organizations, there are a variety of subcultures operating, each with different values and beliefs; it is very common to have a management culture and a staff culture. When subcultures clash, it can negatively affect an organization's performance (Schein, 1992; Schrater, 2008).

Organizational culture theory promotes the idea that a strong and positive organizational culture can foster stability and control within an organization; create consensus among an organizations' employees; promote creativity and innovation that give rise to effective strategies, and their implementation; and lead to strong and steady commitment from employees (McKenna, 1994; Schein, 1992; Shafritz \& Ott, 1992). Attention to organizational culture is particularly important in human service organizations. Employees operating within a positive organizational culture are more likely, and more able, to respond to changes in client needs with innovative suggestions and sustained efforts to implement new ideas that lead to improved client outcomes (Austin \& Hopkins, 2004). If an organization fails to empower employees to use their creativity to assist clients, or fails to give employees the authority needed to provide responses or results for clients, both client service and employee morale will suffer (Schrater, 2008).

According to Lewis, et al. (2001), 
The study of organizations from the standpoint of culture and climate represents a powerful "school of thought"...Because of its potential for the development of valuable insight into the behavior of organizations, especially as that behavior relates to organizational effectiveness, this approach deserves serious study by the student... and by the manager of the human service organization (p. 273).

\section{Learning Organization and Organizational Culture Theories: The Interconnection}

Edgar Schein (1992) defines organizational culture as, "a pattern of basic assumptions - invented, discovered, or developed by a given group as it learns to cope with its problems of external adaptation and internal integration - that has worked well enough to be considered valid and, therefore, to be taught to new members as the correct way to perceive, think, and feel in relation to those problems" (p. 494).

Within an organization, many current behaviors and decisions are predetermined by basic assumptions held by employees. These assumptions are often derived from ideas or activities that worked in the past, and have come to be seen as "the way we do things here." Deeply rooted, but outmoded, beliefs and behaviors can stand in the way of organizational adaptability and innovation. For this reason, it is important for an organization and its staff to carefully and objectively examine basic assumptions on a regular basis (Schein, 1992; Senge, 2006).

Learning organization theory provides both a process and a rationale for consistently examining basic assumptions held by both individual employees in an organization and the organization as a whole (Senge, 1990, 2001). However, if an organization does not have a positive culture that actively supports the implementation of 
the five learning organization theory disciplines, then it is highly unlikely to make any real progress toward a learning culture, and the positive changes such a culture can generate (Lewis, et al., 2001; McKenna, 1994; Schneider \& Smith, 2004; Schrater, 2008; Shafritz \& Ott, 1992).

Managers and supervisors are key to promoting a learning culture. They can model learning behaviors, encourage and reward employees' participation in learning activities, and empower staff to make decisions in their areas of expertise (Austin \& Hopkins, 2004; Senge \& Kaufer, 2000).

Unfortunately, managers often focus on transforming organizational practices, and neglect to transform the organization's culture. For example, a manager may announce that staff will henceforth complete a more comprehensive needs assessment for each client at time of intake (a new practice). However, if the manager fails to provide staff an explanation for the change, additional time to complete the new assessments, adequate training in using the new tool, and a voice in how to implement the change (all of which aid in creating a new culture) staff are unlikely to adapt well to the new practice. As Burud and Tumolo (2004) point out, "The transformation of practices alone... is insufficient and ultimately ineffective if not applied in the context of an adaptive culture" (p. 121).

\section{Learning Organization Theory's Roots in the Business World}

In the first edition of The Fifth Discipline and in two related books, Senge and his colleagues (Senge, 1990; Senge et al., 1994; Senge et al., 1999) primarily focused on the value of utilizing the five learning organization disciplines in private business settings, 
although the potential benefit for hospitals, schools, and governmental agencies was noted. In the revised edition of The Fifth Discipline, Senge (2006) spoke of his expanding awareness of many creative ways practitioners around the world are using learning organization tools and principles in educational settings, governmental and nongovernmental agencies, and community organizations to help workers more effectively address a variety of social problems.

After a 2009 presentation at the Leadership Speaker Series sponsored by a state Department of Personnel in the Pacific Northwest, Senge verified that he had not personally been involved with the implementation of learning organization models in government or human service settings. He said he was aware such efforts have been made in both government and non-profit agencies; he did not believe those efforts have been researched and reported in scholarly journals; and he saw value in doing so (P. M. Senge, personal communication, January 6, 2009).

\section{Learning Organization Models in Human Service Organizations}

In his comments above, Senge implied research on the implementation of learning organization models in human service settings had not been extensive. He would likely concur with Lewis, et al.'s (2001) statement that “reports of empirical studies [regarding the use of learning organization theory] in human services have been rare" (p. 94). A literature search conducted for the purposes of this dissertation supports their views. It revealed that literature relating closely - or even tangentially - to learning organization models in human service organizations is limited and disjointed. The publications that addressed the topic fell loosely into two categories: conceptual models for the 
development of learning organizations in human service settings, and reports on efforts to implement learning organization models in publicly-funded or privately-funded human service practice settings. In some instances, articles incorporated both categories.

Conceptual learning organization models offer a variety of principles and implementation strategies for transforming human service organizations - variously categorized as human service organizations, social service organizations, social work organizations, and public sector organizations - into learning organizations (Austin, 2008; Beddoe, 2009; Gustavsson, 2009; Maden, 2011; Maynard, 2010; Nutting, Crabtree, \& Mcdaniel, 2012; Sherman, et al., 2014). Studies of learning organization model implementation have been conducted within such disparate settings as a child and family service organization, a health department, a health center, a municipality, a library system, several schools or school systems, and a law enforcement agency (Annie E. Casey Foundation Casey Family Services, n.d.; Baldwin, 2008; Beddoe, 2009; Davidson \& McMahon, 2008; Finnigan \& Daly, 2012; Giesecke \& McNeil, 2004; Gustavsson, 2009; Herman, 2007; Hunter-Johnson \& Closson, 2012; Khasawneh, 2011; Lindberg \& Meredith, 2012; Ruffolo, Kuhn, \& Evans, 2006; Somunoglu, Erdem, \& Erdem, 2012) . An overview of the strengths and limitations of learning organization models implemented in human service settings, as described in the literature, is provided below. In addition, authors' suggestions regarding future implementations of learning organization models in such settings are outlined.

Strengths of learning organization models in human service settings. 
The literature suggests that learning organization models have decided benefits for human service organizations. The benefits for individuals and small working groups are that staff are giving a strong voice in a team-based work process; the iterative process found in learning organization models encourages complex communications among team members; staff develop stronger working relationships with coworkers and community partners; and staff figure out more effective ways to do their work in collaboration with coworkers and community partners. All of this fosters both individual and team learning, which adds to the team's knowledge base and helps the team adapt to current and future changes in the workplace. It also fosters individual pride in accomplishment, and increased staff morale (Annie E. Casey Foundation Casey Family Services, n.d.; Beddoe, 2009; Giesecke \& McNeil, 2004; Gustavsson, 2009).

Supervisors benefit from learning organization models in several ways. They learn how to facilitate learning organization processes from outsiders involved in the implementation process; they gain insight into their staff's strengths and interests; and, as their staff gain voice and confidence, they have the latitude to shift their role in the workgroup's project from facilitator to participant. (Gustavsson, 2009; Herman, 2007; Senge, 2001).

Even when learning organization models are implemented at a workgroup level, rather than an organization-wide level, the larger organization stands to gain in several ways. If staff feel more ownership of their work processes, there will be less staff turnover and the organization will benefit from greater staff expertise. In addition, on-site learning experiences are both more effective and less expensive than formal, top-down 
training, so staff will be better-trained and the organization will have lower training costs. Finally, an organization's knowledge base grows when the specific workgroups share what they have learned with the larger organization, making the organization more flexible in negotiating changes. (Annie E. Casey Foundation Casey Family Services, n.d.; Beddoe, 2009; Giesecke \& McNiel, 2004).

\section{Limitations of learning organization models in human service settings.}

While recognizing benefits to implementing learning organization models in human service settings, the literature also delineates factors that can impede such efforts. These include individuals who have difficulty giving up their "expert" or "leader" role, as well as individuals who defer to those they perceive as superior throughout the implementation process. Lack of trust between workgroup members can also be an issue; trust can take months time to develop, and process-oriented activities are negatively impacted until trust exists. (Baldwin, 2008; Lindberg \& Meredith 2012; Ruffolo, Kuhn, \& Evans, 2006).

Some participants in learning organization efforts are not fully engaged because they have plans to move to other employment, or are approaching retirement, and will not be around to see the benefits of the process. Other participants are cynical, and view steps taken to become a learning organization cynically as being "just another fad." Still others, hard-driving or very task-oriented, find themselves frustrated by the pace of a learning organization process (Lindberg \& Meredith, 2012).

For organizations, implementing learning organization models can present a variety of issues. Learning organizations can take years to fully develop, and require a 
great deal of time and energy from key managers and staff in the organization. The training staff need in order to work and think differently can be costly, and new ways of doing business that evolve out of learning organization processes many require changes in personnel policies and job descriptions. Also, cutting-edge ideas generated during learning organization processes may not be well-received by managers in a risk-averse organization (Davidson \& McMahon, 1999).

Sustaining gains made in learning organization processes can also be a problem, particularly if the entire group was not part of the initial implementation, or management does not provide strong and consistent facilitation and support. As previously discussed, even organizations supportive of learning organization models tend to revert to top-down management styles in periods of organizational change or crisis (Baldwin, 2008;

Caldwell, 2011).

\section{Wisdom for future learning organization model implementation.}

The literature argues that human services workers need workplace training based on a learning organization model rather than top-down training, for a variety of reasons. These reasons include: individuals who work daily in uncertain environments need professional development that teaches them critical reasoning; learning and work should not be separate, as more is learned from mistakes than successes; consensus decisionmaking empowers individuals and increases their commitment to the organization, in contrast to imposed learning, which can create resistance; learning together in a work setting allows both individual and team learning to be recognized and rewarded in a timely and genuine way (Beddoe, 2009; Davidson \& McMahon, 1999; Hun ter-Johnson 
\& Closson, 2012). Also, as Lindberg and Meredith (2012) suggest, team projects in the workplace can be an effective way to "harness" the power that lies in the shared compassion that draws workers to public service.

A strong theme in the literature is the importance of supervisor and manager support for learning organization efforts in human service settings. This includes administration's authorization of, and ongoing support for, learning organization efforts. It also includes middle managers' and direct practice supervisors' commitment to and enthusiasm for the effective use of learning organization models; their modeling of learning organization principles and practices; and their consistent mentoring of staff. As giving staff voice is a basic premise in learning organizations, supervisors and managers must be able and willing to invite staff input, and to ask questions inviting multiple points of view. They must then take the time and resources necessary to resolve problems that staff identify. (Annie E. Casey Foundation Casey Family Services, n.d.; Giesecke \& McNeil, 2004; Gustavsson, 2009; Herman, 2007; Hunter-Johnson \& Closson, 2012; Lindberg \& Meredith, 2012; Maden, 2011).

The literature advocates for having clearly-defined and well-managed learning organization processes, during which a specific course is set and then revised as needed. It recommends using pilot programs to prepare for organizational changes, and involving internal and external partners who will be impacted by those changes. It cautions that learning organization processes take time, and that time needs to be intentionally built into employees' workloads. It also warns that such processes require long-term vision, trust among process participants, open and active communication, and a tolerance for 
ambiguity and risk-taking (Annie E. Casey Foundation Casey Family Services, n.d.;

Baldwin, 2008; Bell, 2013; Maden, 2011). 


\section{Chapter 3: Process}

This chapter describes the evolution of the SIA study. The first two sections Background and Preparing for the SIA Study - describe circumstances that existed and events that occurred prior to SIA. The remainder of the chapter discusses the approach I took to various tasks throughout the study, from my initial proposal of the study to VR management to data collection at the completion of each office's SIA project.

\section{Background}

Few social research projects spring suddenly to life, fully formed. The evolution of most studies involves years of effort, examination, and reflection on the part of one or more individuals, institutions or organizations, and finally the serendipitous or intentional "coming together" of parties who share the desire to solve a problem, answer a question, or expand a body of knowledge. In the following pages, I briefly describe the journey that led to the Strengths in Action (SIA) study.

\section{VR's story.}

SIA was conducted in three field offices in a state vocational rehabilitation organization in the Pacific Northwest, hereafter referred to as VR. VR serves people with disabilities who want to work, but face one or more substantial barriers to finding or keeping a job. It provides individualized employment services and counseling to people with disabilities, and technical assistance and training to employers about the employment of people with disabilities. VR is an agency that functions under the auspices of an "umbrella" organization that oversees the majority of social and health services provided by the state (Division of Vocational Rehabilitation, 2010). At the time 
of the study, VR had approximately 300 employees; nearly 250 of those employees were working in 23 field offices throughout the state.

In terms of work processes, VR has a great deal in common with sister agencies in other states. This is because, although agencies such as VR are operated by each state, approximately $80 \%$ of vocational rehabilitation funding is federal, and all state vocational rehabilitation agencies are subject to the same highly specific federal rules and regulations.

\section{Research division studies.}

In the spring of 2007, researchers from the state's research division published two reports examining the decline in VR's rehabilitation rates. They concluded the decline was primarily related to: (a) a struggling economy; (b) a growing proportion of clients with more severe disabilities (served by mandate of the federal Order of Selection, which began for VR in 2000); and (c) a growing proportion of clients on disability-related economic assistance: Social Security's Supplemental Security Income (SSI) or Supplemental Security Disability Income (SSDI). The receipt of disability-related economic assistance was an impeding factor because some clients stood to lose their assistance, including their medical assistance, if they obtained work (Longhi, Wang, Felver, \& Adams, 2007; Longhi, Wang, \& Felver, 2007).

The researchers also noted that certain VR offices had better-than-expected rehabilitation rates (after accounting for differences in local labor markets, disability type, disability grants, and services provided), and concluded this might indicate that key office practices contributed to these better rates. 


\section{The "Learning from Offices" study.}

In the fall of 2007, a small qualitative study, titled "Learning from Offices," was conducted). Four evaluators (two from VR's state headquarters, and two from the research division) visited five high-performing VR field offices. In each office, the supervisor and all staff members were interviewed, and a focus group was conducted, to learn what influenced clients' success at that site.

The evaluators concluded that three areas of "vital behaviors" for client success were: (a) communication (free flow of information; solicitation of input; clearly outlined expectations); (b) commitment to learning and growth (staff encouraged to hone skills and take initiative; goals established by consensus; flexible approach to work); and (c) collaboration/teamwork (staff treated one another with respect; shared expertise; "pitched in" when necessary). This information was shared with staff in participating offices during follow-up visits, and with supervisors and area administrators statewide (Appendix A).

\section{VR's strengths training.}

At the same time the "Learning from Offices" study was taking place, staff at VR's state headquarters were finalizing plans to provide a strengths training course to staff in every VR field office and all specialized units at VR headquarters. This course, based on the Gallup Organization's StrengthsFinder assessment (Rath, 2007) and facilitated by a team of consultants based in the Pacific Northwest, is designed to assist individuals in recognizing their natural talents and their worldview, and in reflecting on 
how to best use their talents and personal perspectives in the workplace and in their personal life.

Before the strengths training began, each VR staff member was provided a copy of StrengthsFinder 2.0 (Rath, 2007); instructed to use a code in the back of the book to access and complete a StrengthsFinder assessment online; and asked to provide the trainers with the results of that assessment (a list of the person's top five strengths) prior to the training.

The training of all VR staff took place during the spring and summer of 2008. Early in each training session, the facilitators provided individual staff members with their StrengthsFinder assessment results: a list of their top five strengths, and a description of each strength. They then engaged the groups they were training in discussions about their personal strengths; how they already used those strengths in their daily lives; and how they might use those strengths to better advantage in their workplace, and in their personal lives, in the future.

During the training, the facilitators repeatedly stressed that individuals can revitalize their work experience if they recognize their strengths and use those strengths to engage in a positive way in their work environment. They also pointed out that organizations are more likely to attain their goals if they help their employees to learn and grow.

At the conclusion of each training session, the facilitators encouraged staff to keep their key strengths clearly in mind, and take them into consideration when making decisions in their workplace. They provided VR's director and selected headquarters staff 
with office-level and individual-level StrengthsFinder assessment results for VR offices statewide. They also provided each VR supervisor with his or her office results and the individual results for each office staff member. As noted above, all trainees received their personal results during the training session (S. Adams and K. Franklin, personal communication, April 21, 2008).

\section{Organizational changes (2008-2009).}

In the year before the SIA study began, two changes occurred within VR's organizational world. The first change, seen throughout the agency as a positive development, was VR's removal from a federal Order of Selection in February 2008 (L. Ruttledge, personal communication, February 8, 2008).

State vocational rehabilitation programs rely on a combination of federal and state funding to operate. When a state program lacks the resources to serve all eligible individuals with disabilities who have applied for services, federal law and regulations stipulate that the program identify in order, by category, which individuals will be served if resources are not available to serve all eligible individuals. The program then moves to what is known as an Order of Selection, a federally-sanctioned waiting list designed to ensure that individuals with the most severe disabilities are served first (Hager, 2004).

An Order of Selection was implemented at VR in 2000. When the order was rescinded in early 2008, after VR substantially reduced its waiting list for services, it meant that VR was no longer mandated to serve the most disabled first, and was therefore free to serve some higher functioning individuals who were potentially easier to place in 
employment settings (S. Adams and K. Franklin, personal communication, March 12, 2008).

The second change VR faced was less welcome, and more difficult to define and manage. Beginning in 2008, a worldwide financial crisis led to serious budgetary issues for many governments, including many states. Like the vast majority of publicly-funded human service agencies in this country, VR found itself contending with serious economic constraints, including a hiring freeze and significant restrictions on staff training and resources. Thus, as the SIA study was being developed, VR faced both an opportunity and a crisis (S. Adams and L. Ruttledge, personal communication,February $8,2008)$.

\section{My role in VR's process.}

I was employed by a state research division in the Pacific Northwest between February 2006 and June 2014. The division operates under the auspices of the organization that oversees the majority of human service agencies in the state, including VR. My work was primarily focused on organization-wide and agency-specific surveys of employees, clients, and providers. In this role, I met and worked with VR's director and a number of program managers at VR's state headquarters.

I was one of the four researchers involved in VR's qualitative "Learning from Offices" study, mentioned above. When analyzing the data from that study, which pointed to the importance of staff communication, learning and growth, and teamwork (Appendix A), I was struck by the parallels between the study results and the principles espoused in learning organization theory. I also recognized that the strengths training 
course VR planned to offer to all VR staff in the spring and summer of 2008 related closely to two of learning organization theory's five disciplines - personal mastery and mental models (Senge, 1990).

When I shared these observations with several of VR's program managers in early 2008 they concurred, and they expressed enthusiasm about the possibility of "piloting" a learning organization model at selected VR field offices subsequent to the completion of their staff's strengths training. They agreed with my assessment that the study I was contemplating would serve as both a natural extension of the strengths training they were offering their staff statewide, and an excellent opportunity to explore the value of implementing a learning organization model in a human service setting.

I was invited to participate in one of VR's upcoming strengths training sessions, and I accepted the offer. In July 2008, I elected to attend a training session put on for VR's Business Services unit, rather than one put on for staff from a VR field office, as I did not yet know which field offices would participate in the proposed study.

Prior to the fall of 2008, my interaction with VR staff was limited to meetings with VR's director and other VR state headquarters employees, with the supervisors and staff at the five field offices involved in the "Learning from Offices" study; and with the staff participating in the strengths training session I attended in July 2008. My subsequent interactions with VR staff, in preparation for the SIA Action study, are outlined in the section below.

\section{Preparing for the SIA Study}


As is the case with most researchers, my preparation for the SIA study was actually the culmination of many years of experience, study, and thought. This section of the report provides a brief overview of the influences that have shaped the worldview I brought to my research. It also includes an outline of the intellectual and practical tasks I undertook, and the decisions I made, in preparation for this study.

\section{My perspective.}

I graduated from the University of Washington with a B.A. in Sociology in the early 1970s. Over the next 30 years, I did casework and supervision in a wide variety of settings, including probation and parole offices, adult and juvenile courts, foster homes, and group homes for individuals with developmental disabilities. I attended numerous conferences and trainings that addressed employee engagement, satisfaction, and performance. I also created and facilitated new employee trainings that focused on these same topics.

As the years went by, I became increasingly aware of a disturbing pattern in the experience of both my coworkers and my colleagues in other publicly-funded social service agencies. Well-meaning individuals would hire into particular organizations because they had the education, experience, and energy to make a positive difference for the population the organization served. These qualities would be noted and applauded at the time of their hiring.

Unfortunately, once on the job, these individuals would quickly discover that administrators in the organization were far more interested in meeting deadlines and production quotas (number of clients seen, number of reports written, etc.) than in the 
quality of their work with clients or their innovative ideas for improving services. Too often, they had no voice in decisions impacting their work; the suggestions they made were ignored or dismissed; or their input was solicited at the office level, but never moved "up the ladder" so meaningful action could be taken.

Tired of being disrespected and disempowered, competent employees often moved on to other jobs. Others remained, defeated by the climate in their workplace, taking little satisfaction in their work, and offering little support to their clients. In many cases, both their health and their spirit suffered. It always seemed to me that, in most instances, small amounts of genuine attention, appreciation, and encouragement could have improved matters greatly for individual staff members and, by extension, their clients.

At the age of 40, having been involved in other work for many years, I returned to Washington State's Department of Corrections (DOC), which had been one of my first employers after graduating from college. I enjoyed my work with adults in that system. I managed to avoid the malaise that affected many of my coworkers for a good number of years, but finally had to admit that my frustration with management was increasing, my energy was waning, and my ability to assist my clients had plateaued. This was one of my primary motivations for pursuing a Master of Social Work (MSW) degree at the University of Washington in Tacoma. Surely, I thought, there must be a more life-giving way for those in the field of human services to approach their work, and a more effective way for supervisors and administrators to support individuals doing that work. 
The MSW program opened many doors for me. Almost immediately, it led to opportunities for me function as DOC's liaison in a variety of community-based programs designed to support DOC clients. Within the MSW program, beyond coursework and internships, I had the opportunity to assist with research and participate in conference presentations of that research. This ultimately led to my interest, and enrollment, in the Ph.D. program at Portland State University's School of Social Work. In the course of my doctoral studies, I developed an interest in learning organization theory, which addresses some of the organizational issues I have been aware of, and concerned about, for years. It speaks directly to the importance of giving staff voice, a chance to learn, and opportunities to do meaningful work with others, with which I wholeheartedly concur. This theory was the focus of my specialization paper (a requirement for my doctoral degree), and is the primary theoretical foundation of my dissertation study.

Entering the SIA study, and throughout the study, I was keenly aware of my personal biases. I believe that most publicly-funded human service organizations make poor use of their employees' energy, creativity, talents, and interests; I want to be part of an organizational process that impacts this situation in a positive way; and I believe a learning organization model that is crafted to reflect, and respect, the complexities and realities of human service settings could go a long way toward helping organizations make the best use of their staff.

My long-held belief (and my personal experience) in empowering staff in human service organizations, coupled with my belief that implementation of a learning 
organization model could generate positive outcomes at VR and beyond, assured that I would bring a good knowledge base, a strong interest, and ample energy to the SIA study. I approached this study knowing I would have to maintain a constant awareness of my biases in order to fairly interpret all I observed and learned. I understood that a variety of factors would impact both my process and my analysis: my education, training, and experience; my connections with the staff and the agency involved in the study; and the very fact the study is designed to explore the potential value of learning organization theory in public sector human service agencies. This study could not be, and should not be, value-free. However, I have done my best to acknowledge, record and report my personal impact on this research, and to assess the evidence accordingly.

\section{Academic endeavors.}

In the decade leading up to the SIA study, I obtained a Master's in Social Work (MSW) from the University of Washington in Tacoma and completed the prerequisites for a Social Work and Social Research doctoral candidacy at Portland State University.

My MSW studies included courses in social work theory and skills, which examined values and practices that serve both clients and workers well; social work supervision, which outlined supervision styles and work environments conducive to engaged and productive workers; social work research, which introduced methods common to studies in the field; and two social work practica. My Ph.D. studies included 
courses in the assessment of, intervention in, and evaluation of social problems; qualitative research methods; and qualitative research methods. In addition, I sought out courses that examined the contemporary U.S. workplace, organizational values and ethics, and survey research, and developed and completed a research practicum that involved interaction with multiple social service agencies.

In the year prior to beginning work on this study, I took on independent studies of learning organization theory and organizational culture theory; examined a variety of potential study methods, ultimately focusing my attention on case studies; read extensively about the advantages and disadvantages of mixed method research; and educated myself about the types of, and benefits of, triangulation. In January 2009, I completed a specialization paper (a requirement of my doctoral program) that examined learning organization theory and its potential value in publicly-funded human service organizations.

\section{Other training grounds.}

While my academic pursuits have been of great personal value, my work experiences have also provided me with information and insights that ultimately led to my interest in learning organization theory and my involvement in the SIA study. While working in the field of corrections, I attended numerous trainings on supervision techniques, staff empowerment and employee engagement, and was struck by how rarely the concepts presented in training were actually put into practice. I developed and 
facilitated various trainings for both coworkers and clients, and could not help but note the energy created when legitimate "voice" was given to either group.

My work with the research division has afforded me a broad and interesting overview of the experience of human service workers. I have been involved in analyzing comments and authoring chapters for four organization-wide employee surveys; more than 10,000 employees, working for a variety of state-funded social service agencies, completed each of those surveys. In addition, I have been involved in developing and reviewing the results of numerous employee surveys for individual state-funded human service agencies. The consistency of the themes that emerge from these surveys is remarkable. Again and again, employees report that they value supportive supervisors, camaraderie with their coworkers, having a strong "voice" in their workplace, and having the flexibility and resources necessary to truly help the clients they wish to serve.

As noted earlier, I participated in one of VR's strengths training sessions in July 2008. This afforded me yet another vantage point on employees' experience in their workplace. Aside from myself, all the staff present at the session worked together in a single VR unit. The tone of the session was relaxed and friendly, and the facilitators of the training were clearly skilled at encouraging even the most reticent participants to talk about their personal strengths.

What I recall most vividly from the strengths training session is this: Despite having worked together for months, or even years, many of the participants shared information about skills and interests their coworkers knew nothing about, and indicated they had not had the opportunity to make use of those skills or interests in their 
workplace. It seemed to me then, and seems to me now, an unfortunate waste of energy and talent.

\section{Pre-study consultations.}

As previously noted, in the spring of 2008, I received encouragement from several VR program managers to focus my dissertation on the piloting of a learning organization model in selected VR offices. Throughout the remainder of 2008, I consulted with mentors and colleagues from several different communities about the value and wisdom of doing such a study, and about practical matters I would need to take into consideration if I moved forward. My advisors included VR staff from field offices and state headquarters; researchers and program managers employed in a variety of capacities at the research division and in other state-funded human service agencies; and mentors and colleagues at Portland State University and the University of Washington.

In January 2009, I attended a presentation by Peter Senge at the Leadership Speaker Series sponsored by the state Department of Personnel. After the presentation, I approached Senge and asked about his experience with the implementation of learning organization models in public- or private-sector human service settings. His response outlined in Chapter 2, Literature Review - influenced my decision to move forward with SIA (Peter Senge, personal communication, January 6, 2009).

\section{Proceeding with caution.}

I was pleased and encouraged by the consensus of those I consulted: the study I was proposing could be of real value to VR and other human service agencies. However, some of the most experienced researchers and organizational leaders I spoke with 
cautioned that research in an organizational setting is fraught with a host of possible pitfalls, including difficulties created by individuals or small groups participating in the study, issues inherent in the organization's existing culture, and problems generated by forces outside the agency being studied. While their cautions did not dissuade me from moving forward with the study, I kept their comments and their advice about how to circumvent potential roadblocks firmly in mind as I moved through the SIA study.

\section{Preliminary Decisions}

While I entered the first stages of the SIA study with numerous unanswered questions, the readings and consultations I had done led me to several preliminary decisions about certain aspects of the study. The most important of these are outlined below, and discussed in further detail in Chapter 4 (Methods).

First and foremost, I concluded that the study I was proposing - an exploration of the implementation of a learning organization model in selected VR offices - would be best accomplished by doing multiple case studies. Second, I determined that utilizing both qualitative and quantitative measures to collect and analyze the data would add to the validity of and confidence in the findings of the study, and that the use of mixed methods would be in keeping with the multiple case studies I proposed.

Third, I recognized that, because some work related to two of Senge's learning organization disciplines - personal mastery and mental models - had already been done by VR staff in a strengths training course, and work related to another of Senge's learning organization disciplines - systems thinking - could not be fully accomplished in the study's relatively short time frame, the study would in fact be an exploration of the 
implementation of a modified learning organization model. Finally, in regard to research questions, I established that I wanted to know if the modified learning organization model had in fact been implemented, which factors had facilitated or impeded the implementation process, what role the participating offices' climate and culture played in the process, and whether there were any notable differences among the offices participating in the study.

\section{Proposing the Study to VR Management}

In October 2008, I met with VR's director and four VR management team members to provide an overview of learning organization theory, discuss how that theory related to current VR circumstances and activities, and propose the implementation of a modified learning organization model in a small number of VR field offices. I explained that learning organization theory can assist organizations in adapting to change, utilizing the resources at their disposal. I suggested that the theory seemed a good fit for VR, which was then, and is now, dealing with both significant organizational change and limited resources. I pointed out that the theory focuses on the use of teams, the use of dialogue, and collaborative learning, all things that VR staff have long been encouraged to incorporate in their work with one another, with their clients, and with community partners.

I provided meeting participants with a handout outlining the specific strengths a study based on learning organization theory would have for VR. Strengths noted included the clear connection between learning organization theory and the findings from VR's "Learning from Offices" study; the consistency between learning organization theory's 
personal mastery and mental models disciplines and VR's recently completed strengths training; and learning organization theory's fit with current literature about effective supervision, and about best practices in vocational rehabilitation settings. Other strengths noted were the opportunity for VR to learn and grow as an organization; the chance for participating staff to learn and grow as individuals; the potential for improved communication among VR staff, and between VR and community partners; the opportunity to collect and use meaningful feedback; and the likelihood that VR clients would ultimately benefit from all of these developments.

In addition, I provided the meeting participants handouts defining the five learning organization disciplines, and the connection each of the five disciplines had to organizational efforts VR had already made, or was currently considering. I then briefly outlined the type of study I was proposing, the preliminary decisions I had made concerning research questions and study design, and the decisions that would have to be made by VR management before such a study could take place in the field.

After listening to my comments and reviewing the materials provided, the management team members shared their opinions of and ideas about my study proposal. In the end, they unanimously agreed that the study had potential value for VR staff and clients, and should move forward.

At this point, the VR director made the following decisions: (a) the four management team members attending the meeting, and a fifth management team member not present at the meeting, would work with me to make the decisions and the arrangements necessary to launch and sustain the study, and (b) one of the four 
management team members attending the meeting would function as my guide throughout the study, making initial contacts with field offices, attending study-related meetings in the participating field offices, acquiring needed resources from agency headquarters, supplying me with information and support, and offering the supervisors of offices participating in the study whatever support they needed. Although neither the group of five individuals assigned to support me nor the individual appointed as my guide were given a formal title, the group of five will hereafter be referred to as the "Planning Committee," and my guide will be referred to as the "Program Manager."

\section{Financing the Study}

I received no compensation for the time I contributed to this study. The hours I spent on study-related activities were separate from the hours I worked at the research division.

The support this study received from VR was the work time taken by the five VR management team members to assist me in developing the study; the noteworthy expenditure of work hours, thought, and energy on the part of the Program Manager who accompanied me and offered me "management's perspective" throughout the process; and the use of a state car so the Program Manager and I could travel together to field office meetings.

The research division supported this study by providing the assistance of a division employee to upload the online survey I developed into SurveyMonkey, a survey software tool that creates and publishes online survey. This was deemed an appropriate 
use of staff time, as VR is one of the state agencies that the research division serves, and the results of the survey would be shared with and stand to benefit VR.

\section{Working with the Planning Committee}

Between November 2008 and May 2009, I met on three occasions with all five Planning Committee members, and frequently connected with one or more of the committee members in person, by telephone, or by e-mail outside of formal meetings. During this time period, a variety of decisions were made about the evolving study. These included defining the roles of those participating in the study, selecting offices to participate in the study, considering the use of incentives, naming the study, and reviewing a variety of study-related materials. Each of the decisions outlined below was ultimately approved by me and all five of the committee members.

\section{Defining the roles.}

When I first met with the Planning Committee members, several of them assumed that I would facilitate the learning organization process in the proposed study. This led to an extended discussion about the importance of such a process "growing" from staff interactions in a natural way, so that the process can be sustained and utilized repeatedly as new issues arise in the office. It was decided that the supervisor in each selected field office would be the natural facilitator of the process. However, there was some concern that supervisors, already overburdened with other tasks and unfamiliar with learning organization theory, might be uncomfortable taking on a new process.

In the end, it was determined that the supervisor in each participating office would be the facilitator of the process. The Program Manager would assume a supporting role, 
assisting in organizing study-related staff meetings, attending all such meetings, and helping in whatever way the supervisor requested. My role would be as an observer of, rather than a participant in, group meetings. However, recognizing the importance of defining my role to all study participants, it was decided that I would assist in introducing the study's purpose and process to the supervisor and staff in selected offices; clarify my role as the evaluator of the process to all concerned; be present at all study-related meetings and selected study-related activities; and prepare an agenda for distribution at the beginning of each meeting (based on the group's progress during the prior meeting). It was also established I would interview each supervisor and selected staff in each participating office, and send an online survey to all staff in each office, at the end of each office's chosen project. (For detailed information on the interviews and the online survey, see Chapter 4, Methods.)

\section{Selecting the offices.}

The most complex and time-consuming decision the Planning Committee was involved in was the selection of the VR field offices to participate in the study. Over the course of three meetings, a variety of office-specific materials were reviewed, opinions were shared, and discussions ensued. Out of this process, three field office "finalists" were selected to be contacted and invited to participate in the study; all three offices accepted the invitation. (For additional information on sampling, see Chapter 4, Methods.)

\section{Considering incentives.}


The use of monetary incentives or other "rewards" for the VR field offices or individual staff members participating in the study were discussed by the committee, but never seriously considered. The primary reason was that no budget existed for incentives. In addition, some committee members expressed concern that offices not selected for the study would already feel participating offices were somehow being "favored," and incentives would exacerbate this issue; other committee members suggested that offering incentives to participate would suggest that offices or staff members were being "bribed" to participate in something burdensome.

I did ask permission to provide refreshments at the first working meeting in each of the participating offices. This met with the approval of the committee members (and later met with many "Thank yous" from supervisors and staff).

\section{Naming the study.}

During the third Planning Committee meeting, it became clear that what we had to that point been calling "the study" needed to have a more formal title before it was introduced to selected VR field offices. Some names were suggested in the meeting, others were e-mailed to me over the next two weeks, and several e-mail votes were taken. By early March, a "winner" emerged from fifteen possibilities: Strengths in Action. It was favored because of its active voice, its brevity, and its reference to the VR staff's recently completed strengths training.

\section{Review of study materials.}


As I developed materials related to the study, I submitted them to the Planning Committee for review. This included the Informed Consent (Appendix B) that would be signed by all study participants; the semi-structured interview questions (Appendix C) I would use in post-intervention interviews; and the online survey (Appendix D) that would be e-mailed to all the study participants at the conclusion of their office's chosen project.

In the cases of the Informed Consent and the interview questions, my intent was primarily to inform committee members of the procedures I would be following. In contrast, committee members were actively involved in the creation of the online survey. (See Chapter 4, Methods, for a more in-depth discussion of the online survey development.)

\section{Ongoing involvement of Planning Committee.}

Once the study commenced, the Program Manager took primary responsibility for keeping the VR Director and the other Planning Committee members updated about the study's progress. This was a very practical decision, as the Program Manager was located in the same office as the director and the other committee members.

One of the Planning Committee members, who was responsible for all VR field operations, had regular contact with the supervisors of the three offices involved in the study, and occasionally visited the participating offices during the study. This individual never failed to inquire about the progress of the study, but did not engage with the study in any more interactive way. As each of the three participating offices completed their study-related project, this same individual sent an e-mail to all staff in the office, 
congratulating them on their accomplishments and encouraging them to take the online survey connected with the study.

\section{Obtaining Authorizations}

In February 2009, VR management provided me with free access to all information strengths training consultants had provided VR subsequent to the 2008 VR strength training sessions. They also granted me permission to access and analyze VR's results from the 2007 Employee Survey in which their staff had participated, recognizing this would assist the Planning Committee in selecting the offices to participate in the study.

On June 4, 2009, I signed a Memorandum of Understanding with VR regarding data access and data sharing. This document specified methods of data access, confidentiality safeguards, ownership of materials, and disposition of data. On June 26, 2009, the Portland State University Human Subjects Research Review Committee approved my application for the SIA study. Shortly thereafter, the Institutional Review Board operating in VR's home state waived review of the study based on Portland State's approval of my application.

\section{Staff Configuration in VR Offices}

In each VR field office, there are three job classifications: Vocational Rehabilitation Supervisor (VRS), Vocational Rehabilitation Counselor (VRC), and Vocational Rehabilitation Technician (VRT). The VRS supervises all staff assigned to

the office; the VRCs provide direct services to VR clients; and the VRTs perform a variety of different tasks to support the VRCs in providing services to clients. 
In a number of field offices throughout the state, one VRS has responsibility for supervising staff assigned to two or more different sites. These staffing groups are technically known as "VR units" rather than "VR offices." However, for the purpose of simplicity and clarity, each VR staffing group supervised by one individual will hereafter be referred to as a VR office.

\section{Approaching the Field Offices}

As noted above, I selected three VR field offices as potential participants in the SIA study, with the assistance of the Planning Committee. (See Chapter 4, Methods, for more in-depth information on office selection.) The remainder of this chapter describes the interactions the Program Manager and I had with the supervisors and staff in the participating field offices over the course of the study.

\section{Contacting the supervisors.}

As soon as it was determined which VR field offices would be invited to participate in the SIA study, the Program Manager placed telephone calls to the supervisors of the selected offices, asking if they would be open to a conversation about the evolving study and their office's potential participation in the study. Each of the three supervisors agreed to an initial meeting.

In late spring 2009, the Program Manager and I had our first meetings with the supervisor in each of the three selected offices. At each meeting, we informed each supervisor of the background to the study, the purpose of the study, their potential role in the study, and their staff's potential role in the study. We explained that the VR Program Manager would be available to support the supervisor during the study, and that I would 
be an overt observer during the study. We also made it clear that all participating supervisors and staff would be provided, and asked to sign, an Informed Consent at the outset of the study; that an anonymous online survey to would be e-mailed to the supervisor and all staff in each participating office when the office completed their chosen study project; that I would interview the supervisor and two or three other randomly selected staff from each office (who consented to be interviewed) when the office completed their chosen study project; and that the study's anticipated time frame was four to six months.

At the end of the meeting, after an active discussion about the proposed study, each of the three supervisors contacted expressed interest in participating in the study. However, each said they wanted to consult with their staff to determine whether they had an interest in participating before scheduling further study-related meetings. The Program Manager and I encouraged them to do so. The first supervisor we contacted asked if I could produce a one-page description of the study to guide discussion about the study with staff. I did so (Appendix F), and the same information was subsequently provided to the other two supervisors.

\section{First meetings with staff.}

Within a month of our initial meetings with the three supervisors, the Program Manager and I were notified by each supervisor that their staff was open to hearing more about the study. We arranged to meet with the staff in each office during one of their regular staff meetings. 
The first meeting with staff followed the same pattern in each of the three offices. At the beginning of each meeting, the Program Manager and I took time to introduce ourselves. The Program Manager, being well known to many of the staff and having helped facilitate previous group processes throughout the agency, needed relatively little introduction or role clarification. Being much less familiar to staff, I took care to explain that I was a student at Portland State University's School of Social Work, and the study was my dissertation project; that I was also an employee at the research division that serves VR and other human service organizations, but that work was independent of my involvement in the study; and that I would be a regular observer of their process, not an active participant in their process, if they chose to be part of the study.

After introductions, the Program Manager and I provided each staff essentially the same information we had previously shared with their supervisor, and answered their questions about the study. We also provided each staff member with a Strengths Profile of their office, and a summary of their individual strengths. (This information came from the strengths training course provided to all VR staff in 2008; the individual strengths information had first been distributed during the training.) We then asked them to take some time prior to the start of the study to reflect on their personal strengths and how they might use those strengths in their workplace, and to reflect on their basic assumptions concerning their chosen work and their role in it.

At the end of the meetings, we asked the staff to consider the information we had shared about the study, to decide together whether their office wished to participate in the study, and to contact us when they had made their decision. One office took less than 24 
hours to respond; another office checked back within one week; and the final office took nearly four months to make their decision, due to events unrelated to the study. In the end, all three offices chose to participate in the SIA study.

\section{Working with the Field Offices}

Throughout the study, every effort was made to follow the same process in each of the three participating offices. This was done so that each office would be operating within the same general framework, even though staff in each office would select and implement their own project and thereby create their own SIA experience. The common steps taken in each office are outlined below.

\section{Role of researcher and Program Manager.}

As previously noted, the Program Manager's role was to attend all SIA meetings in each of the three offices, and to provide the supervisor whatever assistance was wanted or needed to facilitate the project. This role was designed to be flexible, and the degree to which the Program Manager stepped in to facilitate or assist in facilitation of the group meetings did vary somewhat from office to office.

My role was to attend all SIA meetings as an observer of, rather than a participant in, group meetings, and to prepare a written agenda for each group meeting. For the most part, I stayed within my defined role; occasional brief departures from that role are referenced throughout this report. The first agenda I provided to each office was the same for each of the three offices (Appendix G). Thereafter, I created individualized agendas for each meeting based on the progress and the decisions staff had made during their previous meeting. 


\section{Informed consents.}

As the SIA study began in each of the three offices, the supervisor and all staff reviewed and signed an Informed Consent form (Appendix B). The form stated that I would be taking notes on staff discussions, decisions, and interactions during scheduled SIA meetings. It also specified that I would not record statements made by a staff member outside of project meetings (i.e., in an individual's office, or by a water cooler) unless I requested, and received, permission to do so. In addition, it made clear than participants were free to drop out of the study at any time. (See the "Ethical Considerations" section in Chapter 4 for additional information regarding the Informed Consent process.)

\section{Choosing a goal.}

In each of the three offices, the first "working" SIA meeting was spent listing possible goals the office could take on as their SIA project, discussing the merits of each goal put forward, and then selecting a single goal. Staff were asked to choose a goal that would benefit VR staff, benefit VR clients, include some role for all staff in the office, take approximately four months to accomplish, and be measurable (staff would have ways to determine if the goal was met).

In each office, all staff members attended this first meeting, participated to a greater or lesser degree in the discussion about different goals, and stated that they agreed with the goal finally selected. The agenda I provided at the beginning of the meeting 
contained a list of possible goals for VR field offices, but beyond that input neither the Program Manager nor I offered any suggestions or opinions about the goals discussed or selected.

In two of the three offices, the Program Manager did help to facilitate the goal selection conversation, at the supervisor's request. In one office, the supervisor facilitated the entire goal selection conversation; the Program Manager played a secondary role, occasionally offering specific information or answering direct questions posed by the supervisor or staff.

\section{Defining staff roles.}

After choosing the office goal (which was henceforth referred to as the office's "Strengths in Action" or "SIA" project), staff in each office turned their attention to defining the roles each individual would play during the project. One office began this task during their first working SIA meeting, and completed it during their second meeting; the other two offices completed the entire task during their second meeting.

In each office, supervisor and staff first outlined the steps that needed to be taken to reach their established goal. Then decisions were made about which staff member or members would be responsible for completing each step, making certain that each staff member selected a part to play in reaching the goal, and recognizing that staff roles might shift during the project. In addition, staff set a tentative timeline for the office's project, decided how they would communicate with each other about their progress during the project, and talked about how they would know when their goal was met and their project was completed. 


\section{Moving through the projects.}

As the three offices involved in the SIA study moved forward with their chosen project, they continued to adhere to certain common processes. Each office's staff met as a group at least once each month to review project-related activities. During these group meetings, individuals or small groups reported on tasks they had completed; described difficulties they had encountered; shared things they had learned; and put forward new ideas they had for the project.

Staff also used these meetings to discuss and make decisions about whether any adjustments needed to be made to the office's original plan for the project. Whenever a decision was being made about "next steps," the Program Manager (and, in some instances, the supervisor) encouraged staff to take their individual strengths, their shared goal, and the information and insights they had gained in the process to date into account.

Between all-staff meetings, staff worked on a variety of project-related tasks on their own, or in small groups. Many of these tasks involved personal, telephone, or e-mail contact with individuals and organizations outside VR. Throughout the project, staff shared project-related information and ideas with one another in day-to-day conversations, by e-mail, or in scheduled or unscheduled meetings with subgroups working on the same project-related tasks.

\section{Ending the projects.}

When their SIA project was completed, each of the three offices had what can best be described as an "exit meeting." At this meeting, staff informally reviewed what 
had gone well during their project; what roadblocks had been encountered during the project; and whether the project they had undertaken could, or should, be sustained.

Some staff members used the exit meeting as an opportunity to reflect on the value the SIA project had for them, for the office as a whole, or for the office's clients. Others addressed whether or not the process they had used to work through their project could be effectively used when their staff took on other projects, or had to work through specific issues, in the future.

Recognizing that not all staff would feel comfortable sharing their SIA experience in an open meeting, I sent an anonymous online survey to all staff in each of the participating offices as soon as the office's project was completed. All but one staff member completed this survey. In addition, I conducted individual interviews with the supervisor and two randomly selected staff members in each office shortly after the office's project ended; all randomly selected staff members agreed to be interviewed. Detailed descriptions of the methods used in collecting and analyzing observation, online survey, and interview data - including the interviewee selection process - are provided in Chapter 4 (Methods) that follows. 


\section{Chapter 4: Methods}

This chapter describes the methods used in the SIA study. The first section outlines the study's design, including the study's research questions. The second section addresses sampling. These two sections are followed by detailed descriptions of both the methods of data collection and the methods of data analysis employed in the study. The chapter concludes with discussions about developing and maintaining rapport with VR management and study participants, and ensuring rigor and trustworthiness throughout the study.

\section{Study Design}

As discussed in Chapter 2 (Literature Review), there is a notable lack of research and documentation available regarding efforts to implement learning organization models in publicly-funded human service settings. The SIA study explored the process of implementing a modified learning organization model in three VR field offices. The study's goals were to assist in bridging the existing gap in research about the implementation of learning organization models in human service settings; to provide the participating offices with a practical and effective process; and to provide VR policymakers with information that would have utility for the entire organization.

\section{Research questions.}

As outlined in Chapter 1 (Introduction and Overview), the SIA study was designed to answer the following research questions:

1. To what extent was the modified learning organization model implemented?

2. What factors facilitated the adoption of the modified learning organization model? 
3. What factors impeded the adoption of the modified learning organization model?

4. What impact did the adoption (or partial adoption) of a modified learning organization model have on office climate and culture?

5. In regard to the previous questions, what differences, if any, were found among the three VR offices participating in the study?

\section{Modified learning organization model.}

A learning organization model incorporates and attends to the disciplines of personal mastery, mental models, shared visions, team learning, and systems thinking. As noted in Chapter 2 (Literature Review), the implementation of a learning organization model throughout an organization is a complex and layered process that can take years to accomplish.

It is clear, then, that the SIA study was designed to explore efforts to implement a modified version of a learning organization model. The specific modifications were: (a) the study was not agency-wide (only three VR field offices, with 10 to 14 staff each, were included in the study); (b) the study was not long-term (staff in each participating office were asked to select, for the purposes of the study, only one short-term goal that could reasonably be accomplished in four to six months); and (c) strengths training accomplished by staff participating in the study before the study began, and ongoing reflection on that prior training, was allowed to serve as part of the personal mastery and mental models components in this study.

The study design purposely limited the number of offices participating, the study's time frame, the scope of study-related activities, and the study's geographical 
footprint, for three reasons. First, VR was in the midst of a hiring freeze, which burdened existing staff with heavy caseloads and extra responsibilities. Second, due to a poor economy, VR had restrictions on resources available, and involving fewer offices in the study limited the resources required. Third, the Program Manager supporting supervisors during this process faced travel restrictions, so could only participate if the study was neither lengthy nor geographically far-reaching.

\section{Multiple case studies.}

After examining a variety of potential study methods, I elected to utilize a multiple case studies approach for the SIA study. In each of the three participating offices, a case study was conducted that focused on the office's experience in implementing a modified learning organization model over the course of four to eight months.

A case study is a detailed and in-depth description and explanation of the different components of a given social situation. The researcher utilizes as many different data sources as possible relating to the situation being studied. Data gathered is normally largely qualitative, but it may also be quantitative. Tools commonly used to collect data include observations, interviews, surveys, and document review. In the process of analyzing data, the researcher attempts to determine the interrelationships between the various components of the social situation. The goal of a case study is to seek insights, rather than to generalize understanding; these insights may be pursued in subsequent studies. Organizational case studies frequently focus on the introduction of a new practice in the workplace (Babbie, 1990; Berg, 2007, Padgett, 2008; Patton, 2002). 
In the SIA study, the social situation examined was the implementation of a modified learning organization model. The data gathered - primarily, but not exclusively, qualitative - included observations, interviews, and an online survey. In analyzing the data, I looked for interrelationships between different elements of the implementation process in each office. I also looked for similarities and differences among the implementation processes in the three participating VR offices. While the insights that grew out of the study cannot be generalized, they can inform future studies.

Multiple case studies (also referred to as collective case studies, cross-case studies, comparative case studies, and contrasting case studies) make a comprehensive study of several cases. They are considered more robust that single case studies, but still generally use a relatively small sample. The researcher may select cases that are considered "typical," or may choose those that are unique in some way; may select cases that represent a variety of geographical regions, sizes, or other parameters; and may focus on the similarities, or the differences, between cases. Although not required, having a theoretical base can assist in selecting the cases to be studied and - in exploratory studies - specifying what is to be explored (Berg, 2007; Merriam, 2001; Stake, 2000; Yin, 2003).

Three VR offices participated in the SIA study; each was considered a separate "case" in a multiple case study. Offices with specific differences were intentionally selected, so I could examine the similarities and differences in implementing the modified learning organization model in a variety of settings.

Overall, SIA and the multiple case study methodology seemed well-suited to one another. Organizational case studies often focus on new organizational practices, and the 
modified learning organization process was new to VR. Multiple case studies use relatively small samples, and the SIA study was limited to the examination of the implementation experience in three offices. Multiple case studies allow and benefit from the use of multiple forms of data collection and data analysis, and often use both qualitative and quantitative measures, and the SIA study met these criteria. Finally, multiple case studies gather rich and detailed information which can be used by the individuals involved in the project to make meaning of their experience; this was the hope for SIA participants and, by extension, all of VR (Babbie, 1990; Berg, 2007; Padgett, 2008; Patton, 2002).

\section{Sampling}

\section{Type of sampling.}

This study utilized a small purposive sample of three VR field offices. Purposive sampling is a deliberate process of selecting persons, places, or events as research subjects based on their ability to provide needed information (Padgett, 2008). The specific type of purposive sampling used for this study was maximum variation sampling, an approach in which cases that are as different as possible from one another are intentionally selected as study subjects (Patton, 2002).

\section{Selection criteria.}

After careful review of multiple factors, the Planning Committee and I selected three VR field offices for participation in the study. The number of offices in the study was limited to three for reasons outlined in "Modified Learning Organization Model," 
above. None of the three offices selected, or any of their staff, were involved in the 2007 Learning from Offices study in which I participated.

One criterion reviewed during the decision-making process was office location. The Planning Committee and I intentionally selected one office in a large urban area, one office in a smaller urban area, and one office in a rural area. A second criterion reviewed was office configuration. In most VR offices, a supervisor and his or her staff all work in one location; in a small number of VR offices, a supervisor has responsibility for staff at several sites. The Planning Committee and I purposely selected two offices in which the supervisor and staff all work in one location, and one office in which the supervisor oversees staff located at three different sites.

Supervisor strengths (as indicated by the strengths training course results) was a third criterion considered. For each supervisor, the committee members and I looked at whether he or she had any of eleven key strengths (strengths we determined to be particularly valuable for a facilitator of the proposed SIA project) listed among their top five strengths. The eleven strengths were communication (can create clear "pictures" for others, and inspire others to act); empathy (is aware of the emotional tone of a room, and can impact that tone); developer (sees the potential in others, and helps them grow); includer (wants to include others, and makes them welcome); individualization (intrigued by the unique qualities of each person); achiever (sets the pace, and defines the level of production for a group); adaptability (welcomes change, and works well with it); arranger (is a "conductor;" likes complicated situations with lots of variables); connectedness (believes all are connected; helps others see purpose in everyday events); 
futuristic (visionary); and strategic (sorts through clutter, and finds the best route).

Committee members also shared their own perceptions of the particular strengths of each supervisor. We selected three supervisors with different combinations and amounts of these strengths; no two supervisors had more than one of the key strengths in common.

Other criteria taken into account were office climate and culture (as indicated by each office's responses to pertinent questions in the 2007 DSHS Employee Survey); office performance data (as indicated by rehabilitation rates, number of plans written, and other key performance items); staff strengths (office-wide, as indicated by the strengths training course results); office's use of management-sponsored training opportunities (as indicated by training records, and the assessment of VR's Training Coordinator); number of office staff; and each office's proximity to VR headquarters. (The latter factor was a highly practical consideration. Given the economic turndown, VR had travel constraints. Selecting offices too far from headquarters would have meant the VR Program Manager who was designated to support field office supervisors throughout SIA would have been unable to do so.)

An effort was made to select three offices that represented as much heterogeneity as possible. It was reasoned that, in this way, the study's results could point to factors that promoted, or impeded, the implementation of the modified learning organization model across three quite different offices (thus guiding the development of a model that could be tested in additional field offices). At the same time, the study's results could point to similarities or differences in experience among the three offices. 
In sum, the three offices that were chosen to participate in the study have these distinctions: (a) One office is in a large urban area, one is in a smaller urban area, and one is in a rural area; (b) in two of the offices, the supervisor and all staff work in the same location, while in the third office, one supervisor oversees staff in three different locations; (c) for each supervisor chosen, three of their top five strengths were among the eleven key strengths thought to be particularly valuable for an SIA facilitator; no two supervisors had more than one of the eleven key strengths in common; (d) 2007 Employee Survey responses to questions relating to office climate and culture were quite different among the three offices; one office's responses were quite positive overall, one fell into mid-range, and one office's responses were quite negative overall; and (e) performance indicators were also different among the three offices, with one office's results being notably better than the other two offices. (Notably, the office with the most positive responses to survey questions about climate and culture was not the office with the strongest performance indicators.)

After review, the differences in staff strengths, office use of VR training opportunities, and number of staff in each office were not considered in the choice of offices to participate in the study. This was either because there was little apparent difference between the offices, or it was unclear how to compare the information across offices.

\section{Methods Overview}

The SIA study utilized mixed methods; it was a primarily qualitative study that made concurrent use of some quantitative methods of data collection and analysis. 
Altogether, the study utilized three different methods of data collection - two qualitative, and one with both qualitative and quantitative components. It incorporated four methods of data analysis - three qualitative, and one quantitative. The diagram below provides a visual representation of the interconnections among the various methods.

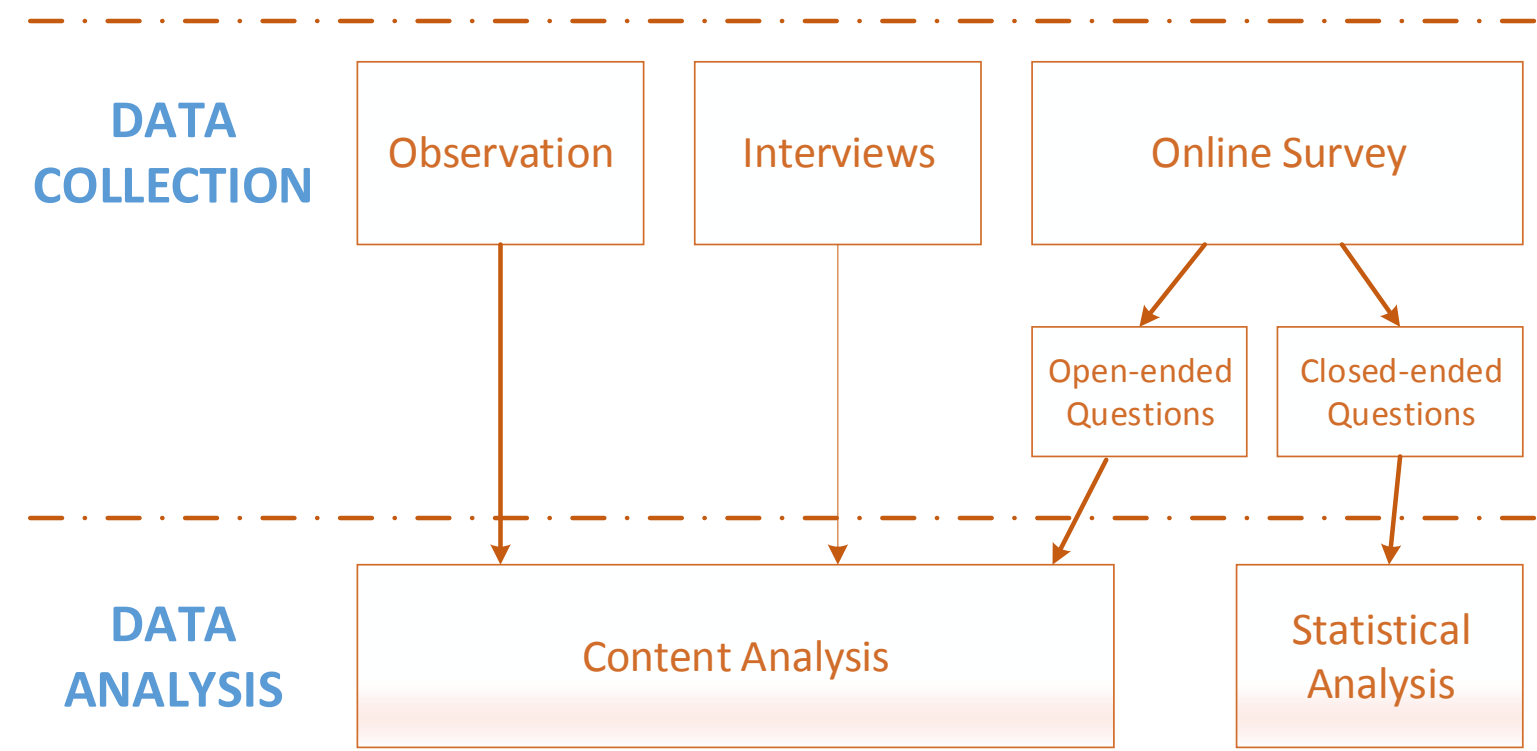

Figure 4.1 - Diagram of interconnections among study methods

Detailed descriptions of each the study's data collection and data analysis methods can be found in the Data Collection and Data Analysis sections that follow.

\section{Data Collection}

Four types of data were collected: observations; interviews; online survey responses to open-ended questions; and online survey responses to closed-ended questions. The observations were done in each of the three participating VR offices throughout the SIA project in that office. The online survey was sent to staff in each 
participating office as soon as the project in that office was completed, and the interviews were conducted in each office shortly after the online survey was completed.

No demographic data were collected during the SIA study. This was a conscious choice on my part, and on the part of the Planning Committee. Given the small number of study participants, analysis of such data could not have been completed and reported in this dissertation without jeopardizing the confidentiality that was promised participants in the Informed Consent (Appendix B) that they signed at the beginning of the study.

\section{Observations.}

\section{Type of observation.}

At the beginning of any field study, a decision needs to be made about whether to enter the field as an overt (announced) researcher or a covert (secret) researcher (Berg, 2007). In the case of the SIA study, covert observation was neither a consideration nor an option. I was only able to gain access to VR field offices by being transparent about my intentions with management, supervisors, and staff.

Fortunately, overt observation has aspects that worked to my advantage

throughout the study. Early on, I was able to locate guides and informants within VR, and they provided me valuable perspectives on what I was observing in each office as the study progressed. Throughout the study, I was able to freely question supervisors and staff in the participating offices if I needed clarification regarding any part of their process. This greatly assisted in collecting critical information, and also worked to increase the level of familiarity and trust between me and the study participants.

\section{Framework for observations.}


As I embarked upon the collection of observations in each of the three participating offices, I fully intended to be as open as possible to whatever I could learn. However, in an effort to focus my thoughts and bring some modicum of structure to what was sure to be a complex reality, I kept the study's research questions, and a number of sensitizing concepts that gave rise to those questions, in mind. These concepts, previously discussed in Chapter 2 (Literature Review), included the five disciplines of learning organization theory (personal mastery, mental models, shared vision, team learning, and systems thinking), organizational climate, organizational culture, and social capital.

\section{Level of participation.}

Observation and participant observation are not discrete processes; they exist on a continuum. Any level of participation in a research process creates the potential for reactivity, which is change due to a researcher's presence. At the same time, too much avoidance of interaction with study participants can lead to the loss of valuable information (Padgett, 2007; Patton, 1990).

While observing the SIA study, I adopted what can best be described as a midlevel of participation. I chose this approach because it would allow me the flexibility necessary in this exploratory study. I also believed it would be the most effective way to get meaningful data about the experience of study participants.

In each of the participating offices, I made certain the staff knew who I was, and why I was present at study-related meetings and activities; I provided staff specific information about the SIA process at the outset of the study; I created written agendas for meetings during the study; and I periodically assisted meeting facilitators in unobtrusive 
ways. However, I did not facilitate the SIA meetings; I did not actively participate in staff discussions and decisions about their office project during those meetings; and I did not offer staff my ideas or opinions about their office project outside those meetings.

During the course of the SIA project, in each of the three participating VR offices, I observed the portions of the office's monthly or semi-monthly staff meetings that addressed the project. (In some instances, the entire staff meeting was set aside to work on the project.) Staff meetings, and impromptu post-meeting conversations with staff, typically lasted one to two hours.

I usually traveled from VR's state headquarters to the staff meeting in one of the three participating offices with the Program Manager. We would typically arrive about fifteen minutes before the meeting began, and spend that time engaged in small talk with the supervisor and staff.

When the meeting began, I would situate myself in a chair in the back of the room, slightly off to one side of the meeting table, so that I had a clear view of the proceedings and all staff involved in those proceedings, but was able to jot notes unobtrusively. Near the end of the meeting, when staff were discussing "next steps," the supervisor or Program Manager would typically ask me if I had anything to add. This was my opportunity to put forward any questions I had about what I had heard during the meeting. I often asked for the meaning of an unfamiliar term, or an explanation of VR's connection to another organization that had come up in conversation. Sometimes I read back small portions of notes I had taken, to make certain I had recorded things correctly. 
I occasionally was asked to, or offered to, step briefly out of my passive observer role. This happened most often during those few project-related meetings the Program Manager was unable to attend. I would assist the supervisor facilitating the meeting by doing such things as writing ideas on a whiteboard during a brainstorming session, handing out printed materials, or agreeing to take down specific information during the meeting and then e-mail it back to all staff.

I often spoke with individual staff members after staff meetings. This was sometimes at my initiative, if I needed to ask follow-up questions, request information, or arrange a meeting. At other times, it was at staff's initiative, if they had information or a question for me. Often the conversations were casual in nature, and related to staff's experiences with the project, the day-to-day activities in their office, or matters totally unrelated to the workplace.

The Program Manager and I used the drive back to state headquarters as an opportunity to compare our impressions of each meeting. I was also able to pose questions raised by the meeting we had just attended that were better answered by someone who had management's perspective.

On several occasions, on a non-staff meeting day, I drove to one of the participating offices to meet with an individual or a small group of staff for projectrelated purposes, or to deliver SIA materials for review. During these visits, I engaged in unplanned conversations with a number of staff members about the progress they were making on their project-related tasks. 
As SIA progressed, and I became more familiar with the supervisor and staff in the three participating offices, e-mails became a common and efficient way to send questions and updates back and forth between meetings. Although useful for all three offices, e-mails were a particularly practical way to keep regular contact with the office located furthest from the community where I lived and worked.

As the SIA project evolved in each office, certain staff members approached me with opinions, suggestions, or concerns about project-related matters that they had not chosen to share with others participating in the project. I consistently encouraged these individuals to take what they shared with me back to the whole group, to the subgroup they were working with, or to their supervisor. In some cases my advice was taken; in some cases it was not. Whatever happened, I never passed on any statement made by a staff member to a supervisor or another staff member, or recorded a statement made without a staff member's express permission

\section{Duration and frequency of observations.}

As the study began, it was anticipated that it would take each of the three participating offices between four and six months to complete their chosen projects. In fact, one office completed its project in approximately four months, another office completed its project in approximately six months, and the last office to complete its project finished in exactly eight months.

The nature of the project chosen by each office had much to do with how long the project ran. The first office to embark on an SIA project chose to create a specific event to which community partners were invited. The date of this event (about four months 
after their project's start date) was set at the same time their project's goal was chosen, and they moved through their process in a timely fashion. The second office to begin a project chose to do outreach to other social service agencies in the community. They initially thought the tasks they set for themselves could be accomplished in about four months. However, they found it took longer to make connections with certain agencies than first anticipated, and their project ultimately took about six months to complete.

The last office to start the SIA project chose to build, or rebuild, very specific connections with another social service agency. Their task was complicated by the fact that their VR office had staff in three different locations; their plan involved all staff in each of their three locations contacting and establishing meetings with all staff in one or more branches of the other social service agency; and the other agency was going through a major reorganization process. Not surprisingly, their project took a full eight months to complete.

Although the offices completed their projects in different periods of time, the hours I spent observing meetings and activities in each office - between 30 and 40 hours - were close to the same. In the office that completed the project in about four months, I spent the most time observing regularly scheduled staff meetings, and some time observing project subcommittee activities. I also attended their project's culminating event, and a project "exit" meeting shortly after the event. In the office that completed the project in about six months, I attended all of the staff meetings, and arranged to return to the office on a number of other occasions to observe or collect information about projectrelated activities that involved only a portion of the staff. In the office that completed the 
project in eight months, I spent almost all my observation time in staff meetings. Because this office was at some distance from my home and work, contacts between meetings were often accomplished by e-mail.

\section{Field notes.}

In the Informed Consent prepared for each participating staff member to sign (Appendix B ), I stated that I would be taking notes on staff discussions, decisions, and interactions during SIA meetings. I also specified that I would not write down any statement made by a staff member outside of such meetings unless I had first requested, and been granted, permission to do so. I adhered to these parameters throughout SIA.

Approaching the study, my intention was to take field notes during SIA meetings or while observing SIA-related activities, and to review and expand upon those notes as soon as possible after periods of observation. Once the study began, I discovered it was much easier to take extensive notes during all-staff meetings, when the participants were actively engaged in conversation with one another and I had no active role in the process. I found it more difficult to tend to note-taking when I was conversing with a single participant, involved in a casual conversation with a small group of participants, or was invited to join a small group engaged in a particular activity. Often, in these instances, the notes that were taken on site were best described as jottings, and were reviewed and expanded after leaving the site.

"Field notes" turned out to be a category that contained an extremely wide variety of information. I always recorded the date, time, and place the notes were taken, and who was present during the meeting, conversation or activity. Beyond that, the information 
collected at any given time included a combination of the following: specific facts, such as the titles of subcommittees participants created, or the names of community partners they intended to contact; descriptions of specific participants, including their appearance, their affect, and their relationships with others; descriptions of social behaviors and interactions that occurred; direct quotes from participants; summaries of conversations which occurred during large meetings or in a more casual context; definitions of terms that were new to me; questions that came to my mind about people, behaviors, processes or policies, that needed clarification in the future; descriptions of and reflections on the “debriefing” sessions I shared with the Program Manager after SIA all-staff meetings; my own feelings and reactions to SIA-related experiences; and insights, interpretations, and ideas that came to me during SIA.

Whatever the extent of my note-taking during a visit to one of the participating offices, I did my best to revisit, reflect upon, and revise the notes I had taken within 24 hours. I assigned each of the study participants a pseudonym. In the beginning, while still familiarizing myself with names and faces, the original notes might contain a person's real initials or even a description such as "short woman, red scarf, spiky blond hair," but the pseudonym was always used in the revised notes. As soon as the real names and associated pseudonyms registered in my mind, I used the pseudonyms exclusively. In revising my notes, I attempted to create concrete and detailed descriptions of the processes and events that were occurring in the participating offices. Although I had originally intended to keep a separate journal of my personal thoughts, feelings and impressions, in the end it seemed to be clearer and more efficient to consolidate the SIA 
experiences and my reactions to them. I handled this by marking my comments and questions about occurrences during the study, or possibilities for the future, as O. C. (for Observer Comments); I marked notes containing my thoughts and feelings as S. R. (for Subjective Reflections). Hardcopies of both the original and revised notes were filed in chronological order in three three-ring binders, one for each participating office. The revised notes were also retained in computer files created for each office. The binders are kept in a locked cabinet in my home office, and the computerized notes are kept in password-protected files on my personal computer.

\section{Interviews.}

In each of the three participating offices, I conducted audio-taped interviews with the supervisor and two staff members shortly after the office's SIA project was completed, and after the study participants had taken the online survey. The interview process, from choosing the type of interview to the transcription of the completed interviews, is described below.

\section{Type of interview.}

I was originally undecided about whether to do individual or group interviews for selected staff members in the participating offices. I ultimately decided to do individual interviews, for several reasons. First, I believed it was more likely I would get honest and thoughtful responses in one-on-one interviews. My extensive experience interviewing staff in human service settings has taught me that, even in small groups, there is always the danger that an individual's voice can be diminished, or drowned, when in the company of others who behave more assertively or are perceived as being more powerful. 
Second, I expected that one-on-one interviews in quiet settings would allow me to better attend to interviewees' comments and body language. Third, I anticipated that extended private conversations with individual SIA participants would provide me the best opportunity to formulate and pose pertinent follow-up questions.

I also had to determine the degree to which the interviews would be structured. I settled on what is described in the literature as a semi-standardized interview format with semi-structured questions. In this approach, there is a pre-determined list of open-ended questions, and all interviewees are asked the same questions in the same sequence. The interviewer is permitted - and expected - to probe during interviews (Berg, 2007; Padgett, 2008; Patton, 1990). In addition to the open-ended questions, I planned to include a small number of questions about the interviewees' employment history at VR, to be asked of each interviewee at the beginning of the interview. The employment history questions would serve two purposes: generating comfortable small talk at the beginning of the interview, and creating some context for observations the interviewee would make in the course of the interview.

I anticipated making liberal use of probes during the interviews, particularly as an adjunct to the open-ended questions. I also intended to allow myself some flexibility regarding the exact wording and sequencing of the open-ended questions, if this assisted the "flow" of an interview. However, I intended to ensure that all interviewees were asked each of the pre-determined questions, to assist in comparison of responses when analyzing interview transcripts.

\section{Developing interview questions.}


I prepared a list of open-ended questions prior to the interviews. I included queries about the positive and negative aspects of SIA, changes that occurred in offices during the SIA projects, what staff and offices had learned from the experience, and SIA's impact on working relationships among staff and between staff and community partners (Appendix C). In doing so, I gave thought to - and noted - the types of probes that might naturally follow each question. I also prepared a short list of employment history questions, which I intended to ask at the beginning of each interview (Appendix C). In formulating the questions, I was careful to avoid double-barreled questions (questions that require a response to more than one issue), complex questions, and questions that would tend to elicit emotional responses from interviewees.

Once the lists of open-ended and employment history questions were completed, I took several steps to preview and pre-test the questions. First, I had three coworkers with years of experience in survey research review and critique the interview questions I had developed, with an eye to both the clarity and the order of the questions. After making several minor adjustments based on their input, I took the revised questions to a meeting of the Planning Committee, who reviewed and approved the questions I had developed. Finally, I conducted mock interviews with two VR employees who did not work in the offices selected to participate in the study, but did have some knowledge of the SIA study that was being planned. These interviews convinced me that the employment history questions were an important and natural lead-in to the more thought-provoking openended questions, and helped me verify my assumption that the interviews would likely take between 30 and 60 minutes to complete. 


\section{Selecting interview subjects.}

I knew from the outset of the study that I wanted to interview the supervisor and two to three staff members in each participating office when the study was complete. I specified in the Informed Consent signed by SIA participants that, in addition to being asked to complete an online survey at the end of their office's SIA project, they might be asked to participate in an audio-taped interview about the project.

In the end, I decided to interview the supervisor, one rehabilitation counselor, and one rehabilitation technician in each participating office. This approach would not take an unreasonable amount of any office's work time, and - in each office - would provide me the perspective of individuals with quite different daily work responsibilities. The selection of the supervisors as interview subjects was purposive, and understood by the supervisors from the beginning of the study. However, I felt strongly - and the three supervisors concurred - that the selection of the staff to be interviewed should be both random and voluntary.

After consulting with the Program Manager and participating supervisors, I settled on a simple process for determining which staff would be interviewed. At the end of the SIA project in each office, I prepared two small boxes. The first box was filled with folded slips of paper, each having the name of one of the office's rehabilitation counselors on it. In the second box, each slip of paper had the name of one of the office's rehabilitation technicians on it. With eyes averted, the supervisor drew two slips of paper from each of the two boxes. The first slip drawn from each box was the person to be interviewed; the second slip drawn from each box was the "alternate interviewee," in the 
event the first person declined to be interviewed. In Office A and Office $\mathrm{C}$, the supervisors chose to complete this process in the privacy of their offices, as they did not want any of their staff members to feel pressured to agree to be interviewed. In Office B, the supervisor chose to complete this process during an all-staff meeting, as she wanted to make certain her staff was convinced the selection was random.

\section{Scheduling the interviews.}

Shortly after it was determined who had been selected to be interviewed in each participating office, I telephoned those I hoped to interview. If it was not already known, I explained exactly how the staff member had been selected for the interview. I underscored that participating in the interview was completely voluntary, and asked if he or she was willing to be interviewed. (All three supervisors had previously agreed to be interviewed; all staff whose names had been first-drawn from the box consented to be interviewed. Therefore, no "alternates" were contacted.) I then told him or her that the interview would be audio-taped, and determined that this met with the interviewee's approval. (All the interviewees agreed to a taped interview.)

At this point, I told the interviewee I expected the interview would last 30 to 60 minutes, and we negotiated an interview time that fit with both our schedules. I asked the interviewee to select - and, if necessary, arrange to use - a private and comfortable space in their workplace for the interview. (All three supervisors opted to be interviewed in their private offices; five of the six staff members elected to be interviewed in a conference room; and one staff member borrowed her supervisor's office for the interview.) I told the interviewee I would send a copy of the open-ended interview 
questions by e-mail, so he or she could review and think about them before the interview, and did so immediately after our conversation ended.

\section{Researcher's advantages.}

As I approached the interviews, I possessed two advantages that increased the likelihood the interviews would be a valuable source of information about the participants' experience during SIA. The first advantage was my experience interviewing in a wide variety of human service settings. Over the years, I have interviewed crime perpetrators, crime victims, family and friends of victims, individuals with mental illnesses and/or histories of abuse, individuals with disabilities, law enforcement personnel, public officials, and many employees in human service settings. These experiences have made me very aware of, and very reflective about, my role as an interviewer. I believe I am skilled at listening well, and being genuinely interested in the interviewee's responses; being friendly, but not over familiar or emotional; and restoring calm to the setting if the interviewee should become agitated. These skills served me well during the SIA interviews, although it must be noted that no agitation surfaced during these interviews.

The second advantage I possessed was the rapport I had already built with the interviewees during the course of the SIA study. By the time the interviews occurred, the interviewees were quite familiar with me, and more trusting of me than they would have been under other circumstances. This promoted free-flowing conversation during the interviews, and allowed me to collect more - and more candid - information than would otherwise have been possible. Also, it is difficult to overstate the benefit of having been 
physically present at, or having personal knowledge of, many of the situations interviewees referenced during the interviews. This provided me context for interviewees' remarks, and it assisted me in formulating clear and productive follow-up questions.

\section{Conducting the interviews.}

When I arrived at a participating office to conduct an interview, I greeted the staff who were present and announced the purpose of my visit. I generally spent a few minutes in casual conversation, "catching up" with the interviewee and other staff, before the interview began. After a short time, I asked the interviewee to direct me to the location chosen for the interview.

Once situated in the interview room, I stated there were a few practical matters I needed to address before the interview began. First, I reviewed the Informed Consent the interviewee had signed at the beginning of the SIA study (Appendix B), with special attention to sections pertinent to the interview. I reminded the interviewee that participation in the interview - like participation in the study - was completely voluntary, and he or she could stop the interview at any time. (None of the interviewees elected to do so.) I reviewed steps I would take to protect the interviewee's confidentiality: the use of a pseudonym in the interview transcript; no use of their name, or their office's name, when reporting the survey results; and safe storage of computer and hardcopy interview data.

Second, I reminded the interviewee that I intended to audiotape the interview, showed them the tape recorder I intended to use, and told them they could request I stop 
taping the interview at any point. (None of the interviewees made such a request.) I then held up a notebook I had brought with me, and asked their permission to occasionally jot notes during the interview. (All of the interviewees granted permission.) At this point, I paused and wrote down the interviewee's pseudonym, the date, and the location of the interview in the notebook.

Third, I explained that I would like to begin the interview with a few questions about the interviewee's employment history with VR (Appendix C), and then move into the open-ended questions I had already e-mailed for their review. I then proceeded to inquire about the interviewee's career with VR. Although I interacted with each interviewee during the SIA study, in most cases I lacked the personal information I collected at the beginning of the interviews. This information included the interviewee's tenure with VR, the length of time he or she had worked in their current VR office, and the different positions he or she had held while working for VR. As I had hoped, these conversations served as an effective "warm-up" to the remainder of the interview, and provided important context for the interviewee's observations regarding SIA.

Fourth, I posed the open-ended interview questions (Appendix C) to the interviewee. I endeavored to adhere as closely as possible to the pre-determined questions. However, in some cases, I made adjustments to the wording or sequencing of the questions to protect the flow of the conversation. In a few instances, a question was deleted because it had already been answered in the course of a response to a previous question. 
At the end of each interview, I thanked the interviewee for his or her time and candor, and stated I would send a copy of the interview transcript for his or her review as soon as it was available. After leaving the office, I took some time (in my car, or in a coffee shop) to record my impressions of the interview in the interview notebook. These notes included information about the interviewee's demeanor, speech patterns, and body language during the interview, and addressed my thoughts and feelings while interviewing. In short, I noted anything I felt would be important to keep in mind during the transcription process.

\section{Transcribing the interviews.}

I personally transcribed the nine interviews. Although this was a time-consuming process, I felt that I would be able to do the most accurate transcriptions, given my familiarity with the interviewees, the terminology used, references made in the interviews, and many of the opinions and feelings expressed by the interviewees. I produced two transcripts for each interview: an initial unedited Word document, followed by an edited Word document.

At the top of the first page of each transcript, I noted the interviewee's pseudonym, the interview number, (e.g., Interview \#3), the date of the interview, and the location of the interview. In addition, in a "Specific Information" section, I recorded how long the interviewee had worked for VR, how long he or she had worked in the current VR office, and the various positions held with VR.

In the first transcription, I included every word the interviewee and I had spoken, including filler words like "um," "like," and "you know." In the second transcription, I 
deleted filler words, and also dispensed with false starts and repetitions. In both transcriptions, I used commas to indicate slight pauses in conversation. I used [long pause] to note longer pauses; I also used brackets to note inaudible words or phrases, laughter, and instances where I intentionally omitted the name of an individual or group. I used capital letters to indicate loud speech, exclamation marks to note emphasis, and dashes at the end of incomplete sentences. Whenever possible, I used standard spelling (e.g., "don't know" rather than "dunno"). Any contextual comments included in the transcripts were both bracketed and italicized.

Once each edited transcript was completed, I e-mailed a copy to the interviewee for review. Seven of the nine interviewees responded that they felt the transcript was an accurate reflection of the interview. Two interviewees suggested minor corrections; in both cases, the corrections related to factual information the interviewee had provided during the interview, and were made before the analysis of interview data began.

Interview tapes and hardcopies of final interview transcripts are kept in a locked cabinet in my home office. All versions of interview transcripts are retained in passwordprotected files on my personal computer.

\section{Online survey.}

\section{Type of online survey.}

Early in the development of the SIA study, I decided to construct a brief online survey to be sent to all staff in the three participating offices. My intent was to send this survey to each SIA participant soon after the SIA project concluded in his or her office, to maximize their recall of the SIA experience and to ensure the voice of all participants 
was heard. I determined the survey would include closed-ended questions about fidelity to the SIA process, learning that occurred on individual and office levels during SIA, and relationships developed (inside and outside VR) during SIA. I also intended to include several open-ended questions addressing participants' perceptions of the SIA process.

\section{Developing the online survey questions.}

At the time of the SIA study, I had several years experience developing both online and hard-copy surveys for state-funded human service organizations. I was quite familiar with the literature on the development of questions for such surveys, and used this knowledge when formulating questions for the SIA online survey.

First and foremost, the literature suggests that an effort should be made to design survey questions that can be administered in a consistent way, and that mean the same thing to all respondents. To this end, it advises that questions should be clear and concise; at a reading level appropriate for the survey's respondents; and free of negative, biased, and double-barreled items (Babbie, 1990; DeVellis, 2003; Dillman, 1978; Fowler, 1995).

In regard to online surveys - especially those measuring opinions, beliefs and attitudes - the literature advises using Likert scales. In such scales, the item is presented in a declarative statement, followed by response options that represent varying degrees of agreement with the statement. The response options should be both exhaustive and mutually exclusive. The use of matrix questions - several questions that have the same set of response options - is recommended for several reasons: they are an efficient use of survey space; respondents will likely find it faster to complete a set of questions 
presented in this fashion; and the format will increase the ease of comparing responses given (Babbie, 1990; Devellis, 2003; Fowler, 1995; Singleton \& Straits, 2005).

Carefully constructed open-ended questions can add value to an online survey. Advantages of such questions are the freedom the respondent has in answering, the wealth and complexity of information that can be collected, and the way in which such questions reveal the respondent's logic and thought processes. The primary drawback to including such questions in a survey is that coding responses to open-ended questions can be a time-consuming and challenging process (Royse, 1999; Singleton \& Straits, 2005).

With the above recommendations and cautions in mind, I developed an online survey that contained three matrix questions. The first matrix question, composed of four declarative statements, inquired about an office's participation in their SIA project; the second matrix question, composed of five declarative statements, focused on what the respondent and his or her office learned during SIA; and the third matrix question, composed of five declarative statements, addressed communication and collaboration among SIA participants, and between participants and community partners, during SIA. The response options for all fourteen declarative statements were the same: Strongly Agree, Agree, Neutral, Disagree, Strongly Disagree. The survey also included three openended questions about the benefits of the SIA process, and ways in which it could be improved (Appendix D).

\section{Previewing and pre-testing the online survey.}

Once I had drafted the online survey questions, I provided a hardcopy to two survey researchers at my place of employment, and e-mailed a copy to members of the 
Planning Committee. I had positioned the four open-ended questions at the beginning of the survey, followed by the three matrix questions, fearing that answering the closedended questions first might influence respondent's answers to the open-ended questions. However, several individuals previewing the survey expressed concern that if the more thought-provoking open-ended questions came first, respondents might not take time to complete the entire survey. As this seemed a valid concern, I moved the open-ended questions to the end of the survey. I also made a few adjustments to the wording of declarative statements based on the feedback I received.

At this point, with the able assistance of a coworker, I launched the survey on SurveyMonkey, a web-based survey tool. I then took the survey myself, and asked three coworkers to do the same, to make certain the directions and questions were clear, the process was intuitive, and there were no errors in the SurveyMonkey system.

\section{Promoting the survey.}

I expected that a high percentage of SIA participants would respond to the online survey, for several reasons. First, historically, VR staff had the highest response rate to online surveys of any publicly-funded human service agency in the state (N. K. Raiha, personal communication, February 16, 2009). Second, I knew I would have the chance to develop rapport with participants before asking them to complete the survey. Third, I knew I would have the opportunity to discuss the survey at the end of the SIA project in each office, and encourage participants to complete it. In fact, during the final all-staff 
SIA meeting in each participating office, I was able to remind staff the online survey was coming, review its purpose, assure them of its confidential nature, and encourage them to complete it.

Even given these advantages, I was aware that formal survey invitations and frequent survey reminders are key to good survey response rates. When the survey link was e-mailed to each participant, it was embedded in a survey invitation that stressed the importance of having input from all SIA participants, and the assurance that survey responses would be anonymous. It also provided my contact information, and encouragement to contact me if there were any problems accessing the survey.

Once the survey was underway, I sent participants a reminder to complete the survey if they had not already done so. The last line of the reminder was, "If you've already taken the survey, thank you! I'm sorry to bother you with this reminder, but - as you know - I have no way to determine who has or hasn't taken the survey.” A second survey reminder - which included congratulations for a job well done on the office's SIA project - was sent approximately one week after the first. In Office A, this reminder was sent by the office supervisor; in Offices B and C, the reminder was sent by VR's director of field operations. In the end, 37 out of 38 SIA participants in the three participating offices completed the survey.

\section{Organizing the survey data.}

Each participating office completed its SIA project at a different time, and staff in each office took the online survey shortly after their project was completed. Because of this, the SurveyMonkey online survey data became available in three distinct groups. 
Thus, it was possible to connect a group of responses to a given office, but was not possible to connect any given response to a particular respondent. As I gained access to responses, I downloaded them from SurveyMonkey and organized them into six different password-protected files: Closed-ended Responses (Office A); Open-ended Responses (Office A); Closed-ended Responses (Office B); Open-ended Responses (Office B); Closed-ended Responses (Office C); and Open-ended Responses (Office C).

\section{Data Analysis}

Two categories of data were analyzed: qualitative data and quantitative data. Qualitative data were collected from three sources: observations, interviews, and the online survey open-ended questions. Quantitative data were collected from one source: online survey closed-ended questions.

\section{Qualitative data analysis.}

Three types of qualitative data were analyzed, in the following order: responses to online survey open-ended questions; responses to formal interview questions; and field notes from observations of SIA staff meetings and staff activities. The same methods of analysis were utilized for all three types of qualitative data, with minor variations. Both the methods common to the analysis of all the qualitative data and the slight adjustments made when coding specific groups of data are described below.

\section{The coding team.}

I recruited two individuals with extensive experience in coding interview and survey responses to assist me in coding the qualitative data collected during the SIA study. Collaborating with co-coders has many advantages. Co-coders can meet 
throughout the coding process to compare notes on their coding of specific sections of data, discuss and defend their coding decisions, negotiate compromises, and refine codes and code definitions. While different coders will always have slightly different "takes" on any given data set - a positive contribution to a data analysis process - frequent communication among coders is a thought-provoking process that serves to increase coders' skills, improve the final product, and create confidence in the study's findings. (Berg, 2007; Charmaz, 2006; Hay, 2005; Lincoln \& Guba, 1985; Padgett, 2008).

In addition to recruiting two co-coders, I asked a colleague at the research division where I was employed to be available for consultation throughout the coding and data analysis process. She agreed, and I made frequent use of her coding expertise in coding and data analysis. Such peer debriefing is a documented method of mitigating researcher bias (Berg, 2007; Padgett, 2008, Patton, 1990).

\section{The response glossary.}

My goal was to create a response glossary (Appendix H) that had a manageable number of clearly defined coding categories, but was comprehensive and flexible enough to "surround" the SIA study's qualitative data. When developing the initial response glossary, I drew heavily on the research questions and interview questions in deciding which codes to include, but was open to adding new codes or and adding new information to flesh out existing codes as the coding process evolved.

In the course of coding the qualitative data, codes were added, when information surfaced that did not fit well into any existing code. In some cases, when the amount of information in a given code was too small or the content was too thin, codes were merged 
with other codes. In other cases, when the amount of information in a given code proved to be large, or the content was layered, codes were divided into sub-codes. Some information surfaced that enhanced existing codes, and was incorporated into the descriptions of those codes. The process of refining the response glossary continued for some time; the glossary evolved into its final form (Appendix H) midway into the coding of the responses to interview questions.

\section{The coding units.}

The coding units for the three sets of qualitative data were slightly different. For the online survey data, one coding unit equaled one response to an open-ended question, whatever the length of that response. (Although there were some exceptions, the responses to the open-ended survey questions did not tend to exceed three or four sentences in length, and were often shorter.)

For the interview data, one coding unit equaled one "chunk" of an interviewee's response to an interview question. When working with this data, the coders started by looking at the entire response to each question posed by the interviewer. From there, if warranted, the coder broke a given response into sections of text that focused on specific thoughts, suggestions, or topic areas. Rarely was a "chunk" larger than a paragraph in the interview transcript.

In the case of the observation data, one coding unit equaled one "chunk" of text from the researcher's field notes. The coders started by looking at a single data entry. From there, the coder often broke the entry into smaller sections of text, much as they did with the interview data. In the case of field notes, a "chunk" of text varied widely. 
Sometimes it was only a word or two; in a few instances, it was as much as a half page of narrative on a single subject (e.g., notes from observation of a supervisor offering support to staff during an SIA activity).

\section{The coding process.}

As a prelude to the coding process, I attempted to provide my co-coders with as much context as possible. To this end, I shared information and materials regarding learning organization theory, the SIA project, and the culture of VR in general and the participating offices in particular. Fortunately, both co-coders were familiar with VR and with the broad outlines of my experiences during the SIA study. Even so, I recognized that my personal experiences during the study afforded me insights into the qualitative data that would be difficult to obtain in any other way. As a result, I often found myself explaining specific terms, or references to specific SIA events, to my co-coders. This provided my co-coders understandings that improved their coding. It also clarified and broadened my thinking about SIA events, which improved my coding and my ability to relate the SIA story.

All three coders elected to use hard copies, rather than computer copies, while coding. This made it easy to list codes and write notes in the margins, and to bring the annotated copies to coding meetings for comparison and review. While coding, each coder also wrote memos - usually no more than one or two paragraphs in length - about ideas that struck him or her while coding, trends emerging from the data, and adjustments that might be made to the response glossary. 
Coding meetings occurred frequently in the beginning of the coding process. Coding deadlines were agreed upon for the completion of specific coding tasks. When the co-coders and I met, we would compare our completed coding. If there were notable disparities in coding units chosen, codes selected, or our interpretation of data, we discussed them until we arrived at a consensus.

Discussions during coding meetings were animated, but cordial. Especially in early meetings, differences in interpretation were common. In some instances, once I provided more in-depth information about a participating office's daily work processes or SIA project, my co-coders came to understand and agree with my coding decision. However, this was not always true. In some cases, a co-coder's interesting perspective on a coding matter caused me to alter or expand my original interpretation, and it was his or her coding decision that prevailed.

A rule established at the outset of the coding process was that coding units could be coded into multiple categories. By no means was every line of text coded, as some was clearly not code-worthy. However, some single lines of text were so rich in content that they were coded in several different categories. In almost all cases, as long as a coder's preferred code was included in the group of codes selected for a coding unit, he or she was willing to agree to the addition of other codes. This greatly aided the coding team's ability to reach consensus regarding particular coding units. Another aspect of the coding process that moved the team toward consensus was the team's ability to adjust the definitions of coding categories as new layers of data, or layers of meaning, emerged. 
The coding meetings also served as an opportunity to look at the "bigger picture" that was emerging from the data. The co-coders and I discussed clear trends apparent in the data. We also noted any data that deviated sharply from the majority of responses we were seeing, and attempted to determine if it was an anomaly or a counter-trend deserving of further attention. (During the coding process, I also shared and discussed emerging trends with colleagues who had agreed to review my survey findings, the Program Manager, supervisors in the participating offices, and my Committee Chair.)

Throughout the coding process, I was in charge of the response glossary. I wrote memos on any changes to codes or code definitions that were contemplated or agreed upon. I also took responsibility for creating new drafts of the response glossary and providing them to my co-coders.

As the coding process evolved and the response glossary began to solidify, faceto-face coding meetings decreased and online coding meetings increased. Midway through coding the responses to the interview questions, personal circumstances severely limited the amount of time one co-coder had to code. From that point forward, one cocoder and I took responsibility for the initial coding of interview and observation data, and relied on the second co-coder to serve as a coding "tie-breaker" when needed.

\section{Defining the SIA "story."}

Once the coding was complete, I began the long and complex process of finding the core of the SIA story in the body of qualitative and quantitative data I had collected and coded. Fortunately, I was able to converse and consult with a variety of individuals who had firsthand knowledge of, or an active interest in, the SIA study. Among those 
who helped me to distill the essence of SIA were my two co-coders; the Program Manager; the supervisors of the three participating offices; colleagues who had agreed to review my survey findings, and my Committee Chair.

\section{Quantitative data analysis.}

A single type of quantitative data was analyzed: responses to online survey closed-ended questions. The analysis methods for this group of data are outlined below.

\section{Responses to online survey closed-ended questions.}

As described in "Data Collection" above, the online survey that SIA participants were asked to take at the end of their office's SIA project included three types of closedended questions. First, respondents were asked to respond to four statements about activities they participated in during SIA (to assess fidelity to the process); second, they were asked to respond to five statements about what they and their office learned during SIA; and third, they were asked to respond to five statements about relationships developed during SIA. Each of the fourteen questions had five response options; the response options were identical for all fourteen questions (Appendix D).

The analysis of the responses to the closed-ended survey questions was primarily descriptive. Frequency and percentage distributions were calculated for each of the fourteen questions. This allowed me to assess - for each question - the degree to which the response of all SIA participants was positive or negative.

However, I was also interested to determine if there was any statistically significant difference in responses to the closed-ended questions among the three offices participating in SIA. To this end, a one-way between-groups analysis of variance 
(ANOVA) and the non-parametric Kruskal-Wallis Test were conducted for each of the 14 closed-ended online survey questions. Two additional tests were run on the one question that approached statistical significance: Levene's Test of Equality of Error Variances, and a Welch Test.

\section{Developing and Maintaining Rapport}

One of the issues uppermost in my mind throughout SIA study was how to establish and maintain rapport with VR management, Planning Committee members, and the supervisor and staff in participating offices. I wanted to create the kind of connections that would elicit the deepest and richest information possible, without compromising my role as an overt observer of the three offices' SIA processes.

Several things served to strengthen my relationship with VR management in general, and with the Planning Committee members in particular. During SIA's development and implementation, I held a Master of Social Work degree, was working toward my Ph.D. in Social Work and Social Research. At the same time, I was employed by a state research division that served VR and all the other social service agencies funded by the state. My educational and professional credentials, coupled with my prior work on multiple VR surveys and VR's "Learning from Offices" study, had several positive effects. They influenced the VR director's decision to allow the SIA study to proceed in three VR field offices; they encouraged the Program Manager to accompany me on the SIA journey; and they made the supervisors who participated in SIA more willing to meet with me early on to learn about SIA and the benefits it might have for their office. 
During SIA, with the invaluable assistance of the Program Manager, I kept management abreast of developments as the SIA study evolved. I also took opportunities throughout the study to tell those who were serving as my VR guides and mentors that their support was truly appreciated. These efforts kept communication flowing, and fostered interactions with management that were both cordial and productive.

The very credentials that smoothed my path with VR management actually set me apart from VR staff during the first SIA meetings. Staff perceived me as being affiliated with VR headquarters, as I came to their office in the company of the Program Manager, who worked at headquarters; I had been part of an earlier qualitative study in different VR field offices; I had the blessing of VR's director to bring SIA to their office; and I was employed by a research division that assists in evaluating their organization. Thus, I was tainted by whatever suspicions staff held about headquarters in general, and headquarters' agenda for promoting SIA in particular.

Fortunately, a variety of factors worked to create trust between me and the supervisors and staff participating in SIA. At the outset of the study, these factors included my providing specific information and answering questions about the study; clarifying the roles that staff, supervisors, the Program Manager, and I would have throughout the study; and establishing, via the Informed Consent, that no individual or office participating in SIA would be named in the reporting of study results.

During the study, the factors that served to increase the connection between me and SIA participants included engaging in small talk before and after meetings; remembering and referring back to things that individuals said or did in prior meetings; 
seeking individual staff members out if I had questions, or needed information, in their area of expertise; and making myself a student of VR terminology and standard VR practices and policies, which allowed me to ask deeper and more complex questions.

Although I made concerted efforts to familiarize myself with VR's world, I understood my knowledge did not begin to compare with that of VR's staff, some of whom had decades of experience with the agency. I shared this awareness at the first working meeting with staff in each of the three offices, and made it clear I would need their help to understand their process clearly. During SIA, I tried to keep Padgett's (2008) advice firmly in mind: "Maintaining a sense of humor, a willingness to be wrong (a lot), and an eagerness to learn is a winning combination" (p. 85). This approach served me well. Staff always provided information I asked for, and frequently stepped forward to share useful information they thought I needed.

One factor that turned out to be more important in establishing rapport than I anticipated was my experience working in systems that VR employees interacted with on a daily basis. This included my work with corrections agencies, juvenile and adult courts, and foster care and developmental disabilities services. Throughout the study, my background allowed me to more easily understand and relate to staff discussions about their work with clients and their interactions with other agencies. In a few instances, I elected to share information during informal conversations with staff that referenced my previous work with public and private sector social service organizations. My experience as a social service caseworker seemed to enhance my credibility in the eyes of staff. One 
staff member remarked, “It's good to know you're not just a researcher. I mean, you've actually done some of the real work, in the trenches."

I am a white, middle-aged woman. I share these characteristics with the three supervisors, the Program Manager, and many of the staff involved in SIA. Our similarities created many natural points of connection. My field notes include references to spontaneous discussions about being a female worker in a large social service bureaucracy, struggling to balance work and family responsibilities, and using parenting skills when interacting with difficult clients. During the study, even casual conversations about strictly personal matters - children, pets, leisure activities - tended to reveal shared experiences or interests. Without question, my having so much in common with SIA participants made building rapport easier than it might have been in other, more diverse, environments.

A final factor that increased staff's trust in me was familiarity. Stoddart's (1986) concept of disattending posits that study subjects tend to quit noticing researchers in their environment over time; researchers gradually "fit in" to the domain they are studying; and, as study subjects come to know the researcher as a person, they suspend their concerns over the research aspects of the researcher's identity. This resonates with my experience during SIA. In each participating office, staff became more comfortable with, and less conscious of, my presence as the study evolved.

\section{Ensuring Rigor and Trustworthiness}

Steinmetz (1991) defines a trustworthy study as one that is carried out fairly and ethically, and whose findings present as closely as possible the experiences of the 
respondents. According to Padgett (2008), threats to trustworthiness include reactivity (when a researcher's presence has a distorting effect on participants' beliefs and behaviors); researcher biases (when a researcher's preconceptions or personal opinions cloud his or her observations and interpretations); and respondent biases (when respondents withhold information, deliberately mislead, or have faulty recall).

\section{Utilizing multiple methods.}

A variety of methods were utilized to ensure the rigor and trustworthiness of the SIA study's findings and mitigate the threats to trustworthiness outlined above. In keeping with Patton's (1990) view that "Triangulation is a powerful solution to the problem of relying too much on any single data source or method, thereby undermining the validity and credibility of the findings because of the weaknesses of any single method" (p. 193), the study made use of:

- triangulation of methods (both qualitative and quantitative data collection and data analysis methods were employed);

- triangulation of data sources (observations, interviews, and an online survey were utilized);

- triangulation of observers (also known as "member checking;" the researcher checked specific project-related information, observations, statements, and impressions for consistency with others - including the Program Manager and participating supervisors and staff - throughout the study, and had interviewees check their interview transcripts); and 
- triangulation of analysts (multiple analysts were used to review online survey, interview, and observation findings).

Two additional methods were used to ensure the accuracy of the study findings. First, I had prolonged engagement with the supervisor and staff in each office. Multiple contacts with research participants over time are known to mitigate both reactivity and respondent bias (Padgett, 2008). Second, I used peer debriefing and support throughout the study. Before the study began, four colleagues affiliated with the state research division where I was employed - each with strong academic credentials, extensive experience in qualitative or quantitative research (or both), and a working knowledge of VR's policies and practices - agreed to review my research process. I kept regular contact with each throughout the study, apprised them of my experiences and my progress, and requested input in their areas of expertise. This approach is known to mitigate researcher bias (Berg, 2007; Lincoln \& Guba, 1995; Padgett, 2008; Patton, 1990).

\section{Addressing social desirability.}

Social desirability is the tendency of respondents to reply in a manner that will make them seem close to "average," and be viewed favorably by others. This form of respondent bias is always a matter of concern in qualitative studies such as SIA (Singleton \& Straits, 2005). In truth, given the multiple work pressures that VR employees were facing as the study began, I anticipated that the "average" staff member's response might be to bemoan rather than welcome a new project, and understate rather than overstate the project's value to VR staff, clients and offices. 
At the completion of each office's chosen project, all staff participating in the project were asked to evaluate the SIA experience in an online survey in which their individual responses could not be traced, although it was possible to determine from which of the three offices the survey came. (This was made clear to all participating staff before the survey was taken.) If they did not trust the anonymity of the survey process, staff had the option of taking the survey from a computer other than their work computer at, or outside of, their worksite. Surveys of this type, which are anonymous and selfadministered through a computer, are recognized as a method that assists in reducing social desirability (McBurney, 1994; Richman, Kiesler, Weisband, \& Drasgow, 1999).

There is no question in my mind that the VR staff who were individually interviewed might, when responding, have had in mind how the information they shared could impact their relationship with their coworkers, supervisor, or other superiors (or, in the case of the supervisors, their relationship with VR management). I attempted to allay each participant's potential concerns, at the outset of the study and again at the start of the interview, by reviewing the Informed Consent used in the study; by underscoring that I did not work inside of VR's world, and my data collection was solely for the purposes of the study; and by assuring them that neither they, nor their office, would be named in any reporting of the survey results. In addition, during each interview, I asked several questions about SIA's impact on the office and the office's clients, as focusing on nonpersonal issues tends to mitigate social desirability (Singleton \& Straits, 2005).

\section{Ethical considerations.}


Research ethics focuses on study participants' informed consent, and protection from risk and harm. It is also concerned with the privacy of study participants and the confidentiality of the research data collected (Berg, 2007; Padgett, 2008; Patton, 1990; Seiber, 1992). I took a variety of steps to assure that SIA would be an ethical study, beginning even before the study design process and continuing throughout the study.

Believing I had a responsibility to understand as much as possible about the participants' work environment, I learned as much as I could about VR's work processes and culture before embarking on the SIA study. When designing the study, I considered ethical standards carefully, and consulted with VR management, research colleagues and my Committee Chair to make certain the safeguards for SIA participants were adequate.

In an effort to ensure all participants' consent was truly informed, I gave them information - verbally, in the Informed Consent, and in other printed materials - before the study began. This included information about the SIA study, what they would be asked to do during the study, and what information they would be asked to provide during and after the study. I also made it known that I was willing to answer questions about consent issues, or any other aspect of the study, at any time. I then gave the supervisor and staff in each office a period of time to discuss the pros and cons of participating in the study before making a commitment to do so.

An Informed Consent form (Appendix B), signed by each SIA participant at the beginning of the SIA project in their office, explained the purpose of the study, and what would be asked of participants during the study. It underscored that participation in SIA 
was completely voluntary, and participants could withdraw from the study at any time. It described steps that would be taken to keep data collected during the study confidential. After they signed the Informed Consent, each participant was given a copy.

I addressed the possibility of economic risk and harm to participants by including a statement in the Informed Consent that conveyed that choosing not to participate in SIA, or withdrawing from SIA at any time, would not affect their employment with VR. The same document addresses social or psychological risk and harm, and the possibility of inconvenience, by clarifying that participants could remove themselves from any SIArelated meetings or activities that made them uncomfortable or impinged on their other work responsibilities.

Although the SIA projects in the participating offices were group efforts, the privacy of participants was addressed in several ways. First, the Informed Consent and other information provided prior to the SIA study described the study and expectations for participants in detail, outlined confidentiality measures that would be taken, and made it clear that participation in the study was voluntary. Armed with this information, if an individual saw the SIA study as an invasion of privacy, he or she could make a choice not to participate. Second, the Informed Consent assured participants that no statement made by a participant outside SIA meetings would be written down unless I first requested, and was granted, permission to do so. Throughout the study, I honored that commitment. Third, each interviewee was specifically asked to select an interview location that afforded adequate privacy. 
As with many qualitative studies, the participants in the SIA study were well known to me and to one another, so anonymity was neither a possibility nor a goal. However, online survey responses were anonymous. I reminded participants of this on several occasions, believing they might be more forthcoming in their responses with such assurances.

I took steps to assure the confidentiality of the survey data, from the point it was first being collected through the data analysis process. These steps included removing both participants' names and offices' names from field notes and interview transcripts and substituting pseudonyms, and removing any other elements that might indicate participants' identities. It also included being careful, while telling the SIA story, not to describe a participant or an incident in such detail it could reveal a participant's identity.

As previously reported, hardcopies of original and revised field notes, and the final transcript of each interview, are kept in a locked cabinet in my home office. The revised notes and all versions of the interview transcripts are kept in password-protected files on my personal computer.

\section{Data Reporting.}

SIA was a primarily qualitative study. Two bodies of data collected - the observations that were made throughout the study and the interviews conducted at the conclusion of the study - were exclusively qualitative. The third body of data collectedthe online survey responses - incorporated both a qualitative and quantitative component.

The quantitative component of the online survey - 14 closed-ended questions with five response options - was included in the SIA study for two important reasons. 
First, as discussed in Ensuring Rigor and Trustworthiness, above, the triangulation of methods is an important way to increase the validity and credibility of a study's findings (Patton, 1990). Second, the survey's closed-ended questions were a straightforward (and anonymous) way for participants to convey their impressions of SIA, even if they elected not to answer the open-ended survey questions.

From the beginning of this study, the expectation was that the SIA "story" would arise from the large and detailed body of qualitative data collected, and that the quantitative data gathered would, or would not, validate and corroborate the qualitative results. The format of the following chapter - Results - clearly reflects this approach. Each research question is answered by first presenting the qualitative results, and then providing quantitative data that compares or contrasts with the qualitative results. 


\section{Chapter 5: Results}

Three VR field offices participated in SIA study. This chapter begins with an overview of the ways in which the three offices (hereafter referred to as Office A, Office B, and Office C) differed, and the characteristics they had in common, both before and during SIA. The overview is followed by a more detailed description of each participating office - its location and circumstances, its history, its SIA project, and its experiences during SIA.

The remainder of this chapter provides the results of data analysis for each of the five research questions. The box immediately below each question contains the storyline that emerged from the data analysis related to that question. The storyline is followed by an outline of data sources analyzed, a data source table, and an explication of the specific elements named in the storyline. At the end of the chapter, key findings relating to each of the five research questions are summarized.

For Research Questions 1-4, discussion of each storyline element begins with a review of qualitative results, followed by an examination of any quantitative results pertinent to the discussion. For Research Question 5, all qualitative results relating to differences among the three participating offices are reviewed first, after which quantitative results regarding the differences among the offices are explored in the "Quantitative Results" section.

Throughout the chapter, staff members' comments - derived from observations, interviews, or the online survey - are italicized. All personal names used in quotes are pseudonyms, and are enclosed in quotation marks. Supervisors and external agencies 
named in quotes are assigned a letter (Supervisor A, Agency Y, etc.). Underlining within quotes is used to denote emphasis.

\section{Overview}

The three offices that participated in SIA were purposely selected because of their differences. These differences included the size and circumstances of the community in which the office is situated (one in a large urban area, one in a smaller urban area, and one in a more rural setting); the physical configuration of staff (two offices with staff at a single site; one office with staff at three sites); the strengths and style of the office's supervisor; and the climate and culture of the offices. During SIA, each office designed and completed a project that reflected the office's specific circumstances, particular needs, and unique way of doing business.

Although the three offices participating in SIA had clear differences, they also had much in common both before and during the SIA experience. Ten of the most notable similarities among the offices are outlined below.

First, each of the three offices is a field office in the same state vocational rehabilitation organization (VR). The three offices operate under, and are accountable to, the same bureaucratic structure.

Second, each office shares VR's mission. The mission is to provide individualized employment services and counseling to people with physical and mental disabilities, and to provide technical assistance and training to employers of people with such disabilities.

Third, each office was impacted by the lifting of the federal Order of Selection (Chapter 3, p. 38). The Order of Selection was implemented at VR in 2000, and was 
rescinded in early 2008. This made it possible for all VR field offices to begin serving higher-functioning clients with more potential for placement in employment settings. It was seen throughout the agency as a very positive development for staff and clients. However, it also presented a real challenge for field offices: how to create the time and the process to find new clients. This challenge was discussed and addressed in each office during SIA.

Fourth, during SIA, each of the offices was feeling the effects of the Great Recession. The economic slump that impacted the nation and the world also had a negative impact on both the staff and the clients in the three offices.

VR staff were somewhat protected from layoffs and severe cutbacks in resources as the majority of the agency's funding is derived from federal, rather than state, sources. However, as VR receives state monies and functions under the auspices of a large state "umbrella" agency, its staff endured the same payroll cuts, furloughs, and restrictions on travel and training as other state human service agencies.

VR clients were also negatively impacted by the recession. Finding employment for individuals with physical and mental handicaps is a difficult task in the best of times, and is harder still when many unemployed individuals without such handicaps are competing for relatively few jobs. Also, while engaged in a process to find suitable work, many clients were struggling to meet basic needs such as food, housing, and medical care.

Fifth, the gender make-up of staff was similar in each office. The large majority of office staff and all three office supervisors were female. The predominance of female 
staff reflected the gender configuration of VR staff statewide. During SIA, 52\% of field office supervisors were female, and $48 \%$ were male. The Program Manager who supported the SIA projects in each of the participating offices was also female.

Sixth, the overall structure of SIA was - by design - exactly the same in each of the three offices. The similarities included, but were not limited to, the supervisor's role as SIA project facilitator; the role of the Program Manager; the role of the researcher; the process of selecting an office goal and defining staff roles; and the format of regularly scheduled group meetings.

Seventh, the supervisors and staff in each of the offices were responsible for managing all of their day-to-day work responsibilities while participating in SIA. This limited the time and energy that could be given to each office's SIA project.

Eighth, although their styles differed, the supervisors actively supported their office's chosen SIA project in both words and deeds. In their role as facilitator, they encouraged staff to follow their lead and engage in the office's project in a consistent and meaningful way.

Ninth, each of the supervisors reported that they found transitioning from their daily role as their office's leader and head decision-maker to their new role as facilitator of their office's SIA project to be an awkward process. Despite this, the supervisors reported finding real value in the SIA process for themselves and their staff.

Tenth, among staff, SIA supporters and SIA resisters were present in all three offices, with the former outnumbering the latter in each setting. The level of support for SIA varied widely among the supporters, with some quite involved and energized in their 
office's SIA project, and others more accurately described as acquiescent. In some instances, a particular staff member's level of support for SIA changed - most often, but not always, for the better - during the course of their office project.

\section{Office A}

\section{Location and circumstances.}

Office $\mathrm{A}$ is located in the heart of a large metropolitan area, some distance from other social service agencies serving the office's clients. Many of those clients are dealing with issues such as homelessness and lack of access to basic resources such as food and medical care, which makes their placement in a work setting more difficult. Some of the office's clients are young adults with disabilities who are preparing to transition from high school settings to job settings. To better serve these clients, the office is working to strengthen their partnership with school districts in the area.

Because the office is situated in a business district, it has the advantage of proximity to a large number of potential employers for the office's clients. However, in recent years, VR has often used paid contractors to function as a liaison between VR and employers. This has resulted in fewer - and weaker - direct VR-employer connections.

\section{History.}

During SIA, Office A's supervisor and some staff shared that the office has a history of conflict between certain staff members that has never been successfully resolved. They also reported the office is staffed with strong individual performers who have, on their own initiative, developed important connections with specific outside agencies and businesses. On the whole, these "strong performers" prefer to work 
independently, which has resulted in little real teamwork within the office and a lack of trust between coworkers. In addition, both supervisor and staff described Office A staff as being a group that are extremely practical and task-oriented, and generally impatient with process.

Although Office A staff were not described as - or observed to be - a strong working group at the outset of SIA, it was clear that some staff members shared warm personal relationships. It also appeared that each staff member had a positive and respectful relationship with the supervisor, who had been in her position for several years when SIA began.

\section{SIA project.}

As a group, Office A determined that a high priority for their office was to increase direct and ongoing contact with employers in their area. The specific goal they established for SIA was to begin building relationships with businesses in their urban neighborhood that were potential employers for their clients.

During the first SIA planning meeting, Office A decided that one interesting - and hopefully effective - way to accomplish their goal was to invite representatives of local businesses to a "business breakfast." They envisioned that during this breakfast they would they would introduce themselves, their agency's mission, their daily work, and the services their agency provides to businesses employing their clients. By the end of the meeting, Office A's supervisor and staff voted unanimously to pursue the "business breakfast" goal. (Of the SIA goals selected by the three participating offices, Office A's 
project was the most rapidly chosen, the most clearly and narrowly defined, and the most quickly and efficiently accomplished.)

\section{Experiences during SIA.}

The supervisor in Office A (hereafter referred to as Supervisor A) was the driving force behind the SIA project in her office. From the beginning, she showed enthusiasm for her office's chosen SIA project, and encouraged her staff's active engagement in the SIA process. She was adept at facilitating SIA group meetings, effectively drawing out the ideas and opinions of reticent staff and diffusing or deflecting less than constructive comments from a few disgruntled staff. Although she welcomed the Program Manager's presence and input during SIA meetings, she was clearly comfortable facilitating the process.

Between SIA group meetings, Supervisor A kept close tabs on her staff's various project-related activities. She made herself available to work with staff on small group efforts, and offered practical and emotional support when staff found tasks they agreed to complete difficult, confusing, or beyond their comfort zone. In short, she was the glue that held the SIA project together, and it was clear her staff respected her and her efforts.

Supervisor A freely admitted that switching from her daily role as the office's leader to her role as SIA facilitator was an awkward process. She often had to stop herself from taking control of parts of her office's SIA project. She tended to handle this situation with humor, publicly scolding herself when she caught herself becoming too directive during SIA meetings. Whoops, there I go again! She also asked each staff 
member's opinion before each group decision was made, and often reminded staff of their SIA goal, and how each person had to play his or her part.

Once the staff in Office A had chosen their SIA project, they self-selected into small committees which were each responsible for a different facet of the business breakfast. During SIA, each committee met periodically as a small group to work on their committee's tasks - such as employer contact, food and decorations, or programming and then reported out on their progress and their roadblocks at all-staff SIA meetings. Both in small group and large group meetings, information shared often prompted discussions that resulted in large or small changes to some aspect of the project.

For an office whose staff described themselves with some pride as "lone rangers," being involved in a common project was a clear departure from business as usual, and it led to a variety of positive developments. In the course of SIA, some staff showed strong support for their coworkers; for example, one staff member took over food planning and shopping when a coworker fell ill. Some willingly (and, in a few instances, unexpectedly) stepped up to assume significant responsibilities; for example, one normally selfcontained staff member took on oversight of all employer contacts. Some who had not had reason or opportunity to work together before discovered they worked well as a team; for example, two staff members with a creative bent collaborated to produce attractive and professional invitations and information packets. Others displayed previously unrecognized skills and talents during SIA; for example, one staff member volunteered to take photographs to document the business breakfast, and her effort was met with welldeserved praise. 
During SIA, staff interactions with local businesses - especially those businesses represented at the business breakfast, but also those businesses that were not - set the stage for new working relationships and sparked many conversations about how continuing outreach to employers could reap benefits for VR clients in the future. At the same time, the connections made with businesses led to a growing awareness that such outreach would require a serious long-term commitment of time and energy on staff's part.

At the completion of their project, Office A's supervisor and staff credited SIA with improving office morale; creating camaraderie; increasing staff communication; strengthening staff relationships; empowering individual staff members to utilize their skills; and helping the office to understand both the benefits and the costs of developing strong and lasting partnerships with local employers.

\section{Office B}

\section{Location and circumstances.}

Office B is located on the outskirts of a medium-sized city. It is situated in a large complex that houses multiple social service agencies. Due in large part to the proximity of large correctional and mental health facilities, the office's clients are often dealing with a variety of issues - criminal histories, mental health issues, substance abuse issues, chronic unemployment, unstable housing situations - that make job placements more difficult. On the plus side, the office is in a community where many potential employers for the office's clients conduct business.

\section{History.}


During SIA, Office B's supervisor and staff reported that the supervisor was relatively new to the office, some staff had recently transferred in (due to the closure of another office), and the establishment of effective working relationships and trust within the office was still a work in progress.

In early SIA meetings, a topic that frequently surfaced was Office B's reputation in VR for being weak in terms of performance, teamwork, and connections with community partners. Supervisor and long-term staff admitted freely that the office's performance measures - including number of clients successfully placed in jobs, timeliness of such placements, and cost of such placements - were not as positive as those of some other VR field offices. However, they felt such direct comparisons with other field offices were unfair. They pointed out that clients they serve struggle with more and more difficult - life issues than clients served by other field offices, and are therefore harder to place with employers; their clients take more of their time, and more of the organization's resources, to place; and one-on-one time spent with challenging clients takes away time that might otherwise be spent developing strong working relationships with coworkers and community partners. They also indicated they did not feel either headquarters or other field offices had a clear understanding of their office's situation.

Of the offices participating in SIA, Office B was the most encouraged by the lifting of the federal Order of Selection. They were pleased to be relieved of the mandate to serve the most difficult clients in their jurisdiction first. They looked forward to finding and serving clients with fewer or less severe disabilities, and less daunting life issues. 


\section{SIA project.}

From the beginning, Office B's supervisor and staff appeared to be in agreement about the office's need to increase, and shift the nature of, its client base. The specific goal they selected for SIA was to contact a variety of organizations in their community including churches, schools, physical and mental health care systems, veterans' services, and programs serving minority groups - that were capable of referring more stable clients to their office. Some of these organizations had worked with Office B in the past, and some had no history of partnership with VR.

During the first two SIA group meetings, some ground rules were established for the office's SIA project. First, each staff member (including the supervisor) would select and commit to contact one or more organizations in the community. Second, "contacting" each organization would include educating key staff in the organization about the mandates of the federal Order of Selection, under which Office B had operated since 2000; the recent lifting of the Order; and how that action freed Office B to serve clients with less severe disabilities and less troubled circumstances in a more timely fashion. Third, the organization would be encouraged to begin referring clients meeting post-

Order of Selection criteria to Office B. Fourth, the staff contacting the organization would take responsibility for maintaining regular contact with the organization in the future.

\section{Experiences during SIA.}

The supervisor in Office B (Supervisor B) initially appeared to be quite skeptical about both the value of the learning organization process and her staff's ability to work 
well together on an office-wide project. Despite this, she welcomed the Program Manager and researcher into SIA group meetings and other SIA-related activities.

Of the three supervisors participating in SIA, Supervisor B made the most effective use of the Program Manager's support. During her office's SIA project, she looked to the Program Manager for information, advice, resources, and assistance in facilitating the SIA process. Not surprisingly, she also voiced the most appreciation for the Program Manager's assistance.

Supervisor B put less energy into daily oversight of her office's SIA project between group SIA meetings than the supervisors of Office A and Office C. She also did less than the other supervisors to promote the concept of small teams working and learning together outside of the SIA group meetings. As a result, most of the outreach efforts made in the course of the office's SIA project were made by individuals rather than groups. However, when certain staff within the office approached Supervisor B with ideas for future small group projects they had envisioned and starting planning during SIA, she was fully supportive of their efforts.

During SIA meetings, Supervisor B did not appear to be comfortable giving up supervisory control, particularly when it came to allowing "voice" to a few staff who held particularly strong opinions. At the same time, she clearly wanted to honor the commitment she had made to the SIA project and the learning organization process. Having a great deal of confidence in and respect for the Program Manager, and being familiar with her skill at facilitation, Supervisor B encouraged the Program Manager to "head up" certain portions of each of the SIA group meetings. During these portions of 
the meeting, she appeared to relax and engage more easily in give-and-take conversations with her staff.

During the first few SIA meetings, a number of the staff members who had worked in Office B for an extended period of time were quite outspoken about the processes in their workplace that were not serving them or their clients well, and methods they felt would work better. The supervisor, who had been in her position for a short time, was clearly frustrated; she felt the long-term staff were dominating the SIA process to the detriment of the newer staff in the office. As meeting facilitator, she attempted to rectify this situation by cutting the comments of the offending staff short, or by failing to respond to complex and heartfelt comments with any follow-up questions. In some instances, her body language - tapping fingers, raised eyebrows, or more subtle body postures that signaled disinterest - made her sentiments clear.

Not surprisingly, as the SIA project evolved, the more seasoned staff who were initially strongly engaged became less so during SIA meetings, and the newer staff's engagement in the process did not increase. The reports staff made in group meetings about their activities in the community were more perfunctory, and led to fewer animated discussions, than reports shared in the other participating offices.

After deciding on their SIA project, each staff member selected the organization or organizations he or she wanted to contact to encourage new client referrals. Although the Program Manager suggested more than once in group meetings two or more staff members could work together in contacting an organization, only two staff "pairs" chose this option. Interestingly, one of the pairs was made up of Supervisor B and an individual 
who had recently transferred into the office, and their interactions with the organization they selected were particularly successful in generating new clients for the office.

Fortunately, the reticence of some staff to engage in group SIA meetings did not extend to their interactions with colleagues in other organizations in the community. During SIA, several staff members spent a significant amount of time with staff in their chosen organizations, and were rewarded with some satisfactory new client referrals. For example, one staff member collaborated with a local church community who had never worked with VR before. During SIA, she received several referrals from the church and its affiliated organizations, had been invited to speak at a church function, and had developed a system to keep regular contact with church staff.

When their SIA project ended, Office B's supervisor and staff indicated that participating in SIA was valuable for their office. They spoke of the way in which the SIA process encouraged them to take time to reach out and meet with staff in other organizations, an effort which had already netted them stronger relationships with staff in other agencies and some much-needed new client referrals. Some staff reported they intended to work together in the future with one or more of their coworkers on related projects that they had envisioned and begun to develop during SIA. These fledgling projects had the full support of their supervisor.

For Office B, the clearest benefit of the SIA project was closer ties to other community organizations, and the new client referrals those ties would generate over time. Several staff indicated that, in retrospect, they felt they and their office would have benefited if their office had taken a stronger "team" approach during SIA. 


\section{Office C}

\section{Location and circumstances.}

Office $\mathrm{C}$ is actually three offices, each located in a different small community in a rural area, who share a single supervisor. One office is co-located with other social service agencies that serve the community; one is located near the other social service agencies in the community; and one is located at a distance from such social service agencies. Office C's clients tend to have fewer mental health, substance abuse and housing issues than the clients served by Office A and Office B. However, as the three offices that comprise Office $\mathrm{C}$ are all located in small communities, there are a limited number of potential employers and social services available to the office's clients.

\section{History.}

Office C's supervisor has a long history working in the same agency and same geographical area, first as a counselor and then as a supervisor. The majority of Office $\mathrm{C}$ staff have worked together for many years. Although staff are divided between three different physical sites, they have a history of supporting one another. When one site is temporarily short-staffed, an individual from another site often fills in. When a natural disaster struck one site, staff from all three sites worked together to repair the damage to the VR office and to serve the site's clients elsewhere until things returned to normal.

During SIA, Office C's supervisor and staff spoke frequently of the comfortable connection among the three sites. They said this positive relationship grew out of working together for years, the trust that had grown over those years, and the experience of supporting one another during difficult times. They also spoke often of the strong 
connections staff had with other social service agencies in the area, which they saw as a natural advantage of working in small communities.

\section{SIA project.}

The supervisor and staff in Office C recognized the need to work "smarter" during difficult economic times, and believed that one way to do so was to coordinate more closely with other agencies offering services to VR clients. The specific goal they set for SIA was to collaborate with a particular social service agency (hereafter referred to as Agency S) to streamline services to common clients in three communities Office C serves. The reason Agency S was chosen was two-fold: it serves many of the same clients served by VR, and it was going through major organizational changes at the time of SIA that could potentially impact Office C's clients.

As noted above, Office C's staff are located in three different physical sites. In one site, Office $\mathrm{C}$ is housed in the same building as Agency $\mathrm{S}$; in one site, Office $\mathrm{C}$ is located a few blocks from Agency S; and in one site, Office $\mathrm{C}$ is located some distance from Agency S. The differences in proximity influenced how the staff in each site chose to interact with Agency S during SIA.

In the Office $\mathrm{C}$ site housed with Agency $\mathrm{S}$, a meeting of all VR staff and all Agency S staff was arranged. The meeting included discussion of the process Agency S needed to follow when referring clients to VR, the information Agency S needed from VR regarding common clients, and specific changes that would improve collaboration between the two agencies. From that point to the completion of SIA, there was a notable 
increase in interactions and information-sharing between Office $\mathrm{C}$ and Agency S staff on a daily basis.

In the Office $\mathrm{C}$ site which was situated a short distance from Agency $\mathrm{S}$, the connection between the two organizations was less formal, but no less successful. The VR staff - who had previously been co-located with Agency S staff, and knew them well - contacted Agency S staff one-on-one, by telephone or in person, and explained needed changes in VR's referral process. Agency S staff, in turn, asked VR for changes necessitated by Agency S's reorganization. Over the course of SIA, continued conversations led to a gradual simplification and improvement of information-sharing between the two organizations.

In the Office $\mathrm{C}$ site that was physically further from Agency $\mathrm{S}$, the connection and attempted collaboration with Agency S was somewhat more problematic. Agency S staff were overburdened with responsibilities connected to Agency S's reorganization, and it was difficult to establish mutually agreeable meeting times. However, during SIA, the site's staff managed to contact Agency S staff by telephone and e-mail on several occasions. In addition, Office C's supervisor met one-on-one with Agency S's supervisor and administrators, and received assurances that Agency S staff would work together with this specific Office C site in the near future to better serve the two organizations' common clients.

\section{Experiences during SIA.}

The supervisor in Office C (Supervisor C) was slower than the other supervisors to commit to participation in SIA, both because of initial reservations about the time 
involved and because she wanted to make certain her staff and her superiors supported the effort. Once committed, she willingly - but tentatively - assumed the role of facilitator in the SIA group meetings. She made the Program Manager the focus of the first two meetings by referring to her as an "expert" and a "guide," asking for her input, and inquiring about information or resources headquarters might provide to Office $\mathrm{C}$.

Thereafter, Supervisor C took on an active role as SIA facilitator, and paid particular attention to giving each of her staff voice in the process. As the staff became more engaged in the office's SIA project, she granted them more and more of a lead in conversations during SIA group meetings. Her body language signaled this change; she would lean back in her chair, or actually move a bit back from the table, listen intently, and let the conversation flow. When illness forced her absence from an SIA meeting, she appointed another staff member to fill her role, and told the Program Manager she was comfortable doing so.

Supervisor C played a key role in the office's SIA project, serving as the primary liaison between her staff and Agency S supervisors. However, as her staff took more ownership of the project, she gradually assumed a lower profile in the day-to-day interactions between the two organizations. In taking this approach, she very intentionally - and quite effectively - granted her staff a stronger and stronger voice in the SIA project.

As previously noted, Office C staff does their daily work at three different sites. Once the SIA project was defined by the larger group, the staff quite naturally fell into three smaller SIA working groups, each comprised by site. Between monthly large group meetings, the smaller groups established contact and began collaborating with the 
Agency S staff working in their community. As noted above, the staff at each site took a somewhat different approach to their interactions with Agency S.

From the first SIA large group meeting, it was apparent that Office C's supervisor and staff have an "easy" way of interacting with one another. Throughout the SIA project, the majority of staff were actively engaged in discussion about the SIA process. At each all-staff meeting, time was taken for each smaller group to describe their contacts with Agency S. When a staff member from one site shared a positive experience, staff from other sites would affirm them and, in some instances, learn from them. That's great! Maybe we should try that, too. When someone shared a struggle they were having, others were quick to offer possible solutions.

As the SIA project evolved, staff reported benefits they were seeing from their increasing interaction with Agency S. Staff at the Office C site co-housed with Agency S said key information regarding common clients was being shared more frequently and efficiently, with benefits for both organizations. They also indicated that increasingly friendly daily interactions with Agency S staff had changed the "tone" of their workplace. Staff at the Office $\mathrm{C}$ site located near Agency $\mathrm{S}$ were convinced their clients were better served because more information was flowing between the two organizations. They also said they were enjoying renewed connections with Agency S staff they had known for years, but had interacted with infrequently. Staff at the Office C site located some distance from Agency $\mathrm{S}$ were clearly frustrated by their lack of progress in connecting with Agency S. However, as SIA came to a close, they were encouraged that Supervisor 
C had made contact with the Agency S supervisors, and hopeful that their contact with Agency S would increase in the near future.

Office C's supervisor and staff felt the energy they spent collaborating with Agency S was well spent, as their improved communication with Agency S staff allowed them to work more efficiently and gave their clients access to more - and more timely services from both agencies. They fully expected their collaboration with Agency S to continue beyond SIA, and to become even more effective for both organizations in the future.

For Office C's staff, another clear benefit of SIA was the shift in communication patterns within Office C. One staff member interviewed at the conclusion of the SIA project explained that staff from the three Office $\mathrm{C}$ sites had always gotten along well, enjoyed connecting at monthly staff meetings, and willingly assisted one another in times of trouble. However, up until the SIA project, the monthly staff meetings were primarily a vehicle for the supervisor to keep staff updated on agency processes and mandates. The interviewee reported that process of each staff reporting out on his or her activities and offering information and advice to staff from other sites, which started during SIA, was continuing beyond SIA. She said that staff from the three sites now used that same process at each of their monthly meetings to share information and advice with one another, and it was working to everyone's advantage. 
Research Question 1: To what extent was the modified learning organization model implemented?

\section{Storyline for Research Question 1}

The modified learning organization model was implemented in each of the three offices that participated in the Strengths in Action project. In each office, the majority of staff engaged in all five learning organization disciplines - personal mastery, mental models, building shared visions, team learning, and systems thinking.

Figure 5.1 - Storyline for Research Question 1

\section{Data Sources}

The four data sources analyzed to answer Research Question 1 were observations, interviews, online survey open-ended questions, and online survey closed-ended questions. Table 5.1, below, summarizes which data sources contained evidence for which of the five learning organization disciplines.

\begin{tabular}{|l|c|c|c|c|}
\hline & Observations & Interviews & $\begin{array}{l}\text { Online Survey } \\
\text { Open-ended } \\
\text { Questions }\end{array}$ & $\begin{array}{l}\text { Online Survey } \\
\text { Closed-ended } \\
\text { Questions }\end{array}$ \\
\hline Personal Mastery & $\mathrm{X}$ & $\mathrm{X}$ & $\mathrm{X}$ & $\mathrm{X}$ \\
\hline Mental Models & $\mathrm{X}$ & $\mathrm{X}$ & $\mathrm{X}$ & $\mathrm{X}$ \\
\hline Building Shared Visions & $\mathrm{X}$ & $\mathrm{X}$ & $\mathrm{X}$ & $\mathrm{X}$ \\
\hline Team Learning & $\mathrm{X}$ & $\mathrm{X}$ & $\mathrm{X}$ & $\mathrm{X}$ \\
\hline Systems Thinking & $\mathrm{X}$ & $\mathrm{X}$ & $\mathrm{X}$ & $\mathrm{X}$ \\
\hline
\end{tabular}

Table 5.1 - Summary of Data Sources - Research Question 1

\section{Overview}

As outlined in Chapter 2 (Literature Review), learning organization theory is composed of five learning organization disciplines - personal mastery, mental models, building shared visions, team learning, and systems thinking. At the outset of the study, it was established that the SIA study would be an attempt to implement a modified learning organization model. The primary reasons for the modified designation were: SIA was not agency-wide (only three VR offices participated in the study); SIA was not long-term 
(the three participating offices completed their chosen project in four to eight months; literature suggests it can take an organization years to master systems thinking and become a fully-evolved learning organization); and all but one SIA participant had completed an agency-sponsored strengths training course prior to SIA which specifically addressed two of the five learning organization disciplines - personal mastery and mental models.

In order to answer Research Question 1, qualitative and quantitative data gathered during SIA were analyzed through the lens of the five learning organization disciplines. The data analyzed contained the strongest evidence for team learning, with attendant evidence for building shared visions (the precursor to team learning). There was also evidence that some staff in each of the three participating offices continued to develop personal mastery and mental models during SIA. In addition, although SIA did not last long enough for systems thinking to mature, data analysis pointed to the emergence of that discipline in the participating offices.

The remainder of this section on Research Question 1 is a detailed discussion of the extent to which the modified learning organization was implemented, utilizing evidence of each of the five learning organization disciplines: personal mastery, mental models, building shared visions, team learning, and systems thinking. A comprehensive outline of the discussion about Research Question 1 findings is provided in Figure 5.1, below. 


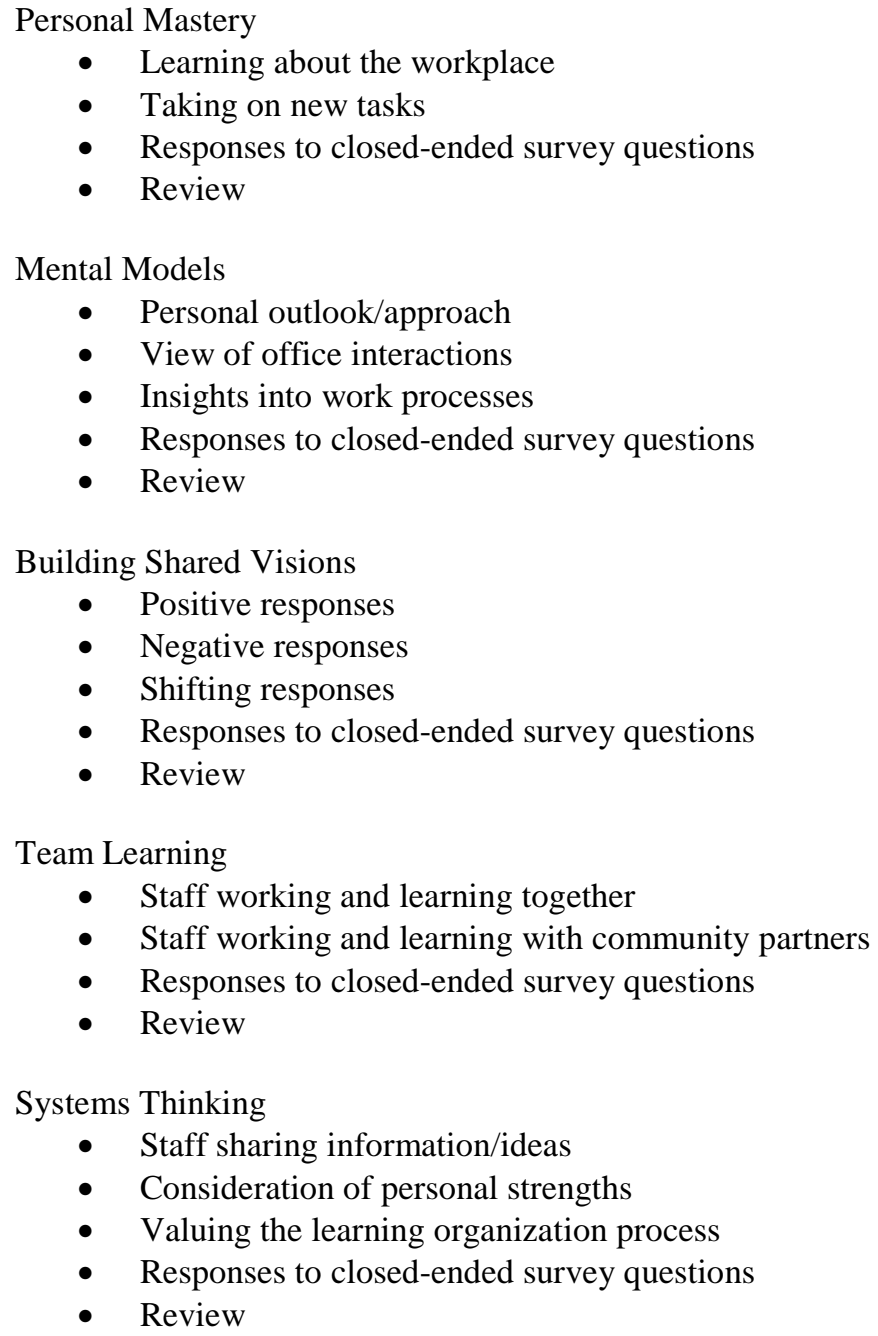

- Learning about the workplace

- Taking on new tasks

- Responses to closed-ended survey questions

- Review

Mental Models

- Personal outlook/approach

- View of office interactions

- Insights into work processes

- Responses to closed-ended survey questions

- Review

Building Shared Visions

- Positive responses

- Negative responses

- Shifting responses

- Responses to closed-ended survey questions

- Review

Team Learning

- Staff working and learning together

- Staff working and learning with community partners

- Responses to closed-ended survey questions

- Review

Systems Thinking

- Staff sharing information/ideas

- Consideration of personal strengths

- Valuing the learning organization process

- Responses to closed-ended survey questions

- Review

Figure 5.2 - Outline of discussion of Research Question 1 Findings

\section{Personal Mastery}

Personal mastery is taking the time, and intellectual and emotional effort, to clarify, and then deepen, one's personal vision. In essence, it is an individual figuring out what he or she considers truly important (Senge, 2006; Senge, Kleiner, Roberts, Ross, Roth, \& Smith, 1999). Individuals striving for personal mastery are notable for their 
desire to learn, to continually increase their proficiency in their work, to gain expertise they can share with others, and to find creative ways to attain desired results (Senge, 2001, 2006).

Prior to SIA, all but one staff member participating in SIA attended an agencysponsored strengths training class that focused on personal mastery, and completed a strengths assessment prior to the class which defined his or her top five personal strengths. (The one staff member hired after the class was held was provided - and completed - the strengths assessment used in the training prior to the start of SIA.) The trainers underscored that employees and the organizations they work for benefit when employees are encouraged to recognize and use their particular strengths in the workplace.

At the first SIA meeting in each of the three participating offices, staff received and reviewed a copy of their top five personal strengths, as determined in the strengths training class they attended. They were also provided a copy of their office's overall strengths profile.

During SIA group meetings, staff frequently shared things they had experienced while doing project-related tasks, and things they had learned about people and organizations outside VR who were positioned to help or hinder the office's SIA project. Agency S has been difficult - they're in the middle of restructuring right now, and their social workers are being shuffled around. They want to work with us, though. The interviews and online survey conducted at the completion of SIA pointed to other, more subtle, forms of personal learning that had taken place in the preceding months. These 
included individuals learning about how their workplace functioned, and individuals taking on new tasks. The "new tasks" category often led individuals to move out of their comfort zone, develop skills, exhibit strengths or talents not previously used in the workplace, or take on more responsible roles in the office or in the community.

\section{Learning about the workplace.}

Some staff spoke of their growing awareness of their workgroup's ability to function as a team. I learned that actually, as a team, we can pull together. While generally positive, a number of comments also conveyed that team projects such as SIA require real engagement and effort on the part of participants. It's not a traditionally perfect team, but I think when push comes to shove, everybody did their part, everybody did what was expected of them, some went further, and people really were concerned about the outcome.

In some cases, the learning about the workplace involved an increased awareness of certain coworkers - knowing more about them on a personal level, recognizing their particular strengths or weaknesses, or coming to understand how to work effectively with them. Some comments in this area were quite specific. That turned out to be a big job for the two of them, to kind of pull that committee along... they had to get a little assertive with people, and that was interesting to watch. Other comments were more general in nature. I learned something about every single person. Overall, the comments suggested that the SIA experience increased staff's awareness of their coworkers' strengths, weaknesses and worldviews. 


\section{Taking on new tasks.}

Many staff members participating in SIA agreed to take on tasks they had not done before. Some tasks - such as setting up event spaces, or producing brochures - were accomplished within the workplace. Many tasks - such as contacting employers or meeting with staff in other organizations - required staff to learn or revisit information about VR processes and policies, in order to make clear presentations to others in the community.

Staff frequently commented on ways in which the SIA experience deviated from their "everyday" work experience. This project enabled me to work outside my normal job duties and do more community outreach. Several suggested they welcomed the change of pace, and the opportunity to do something positive for their agency. I got out of the office and interacted with potential referral sources, and increased community awareness of VR. Some indicated that the tasks they chose to take on for the SIA project forced them to give more thought to, and spend more time on, being an effective ambassador for VR and building long-term connections in the community. I went to the extra effort to contact different Agency $R$ staff directly...I'm doing more of it, a lot more of it, than I was.

Not all staff were initially - or even ultimately - comfortable in taking on an unfamiliar role inside or outside their workplace. It forced people maybe a little bit out of their comfort zone, to go out and contact people. Some participants felt SIA was just the impetus staff needed to gain a different perspective on their work. It really pushed people to scan their horizon. Other participants approached new tasks with some uneasiness, but 
ended up with a sense of accomplishment. I got out of my comfort zone, and found a new agency to connect with in the community.

Altogether, the majority of SIA participants reported feeling positive about engaging in new behaviors, and some believed the willingness of most staff to move into uncharted territory spoke well of the team as a whole. Most of us had a sense of humor when it came to doing something out of our comfort zone, and that shows that we are a team that is fully committed to the idea of the end result and making it happen, one way or the other.

\section{Responses to closed-ended survey questions.}

The responses to five closed-ended online survey questions included in Table 5.2, below, are pertinent to the discussion of personal mastery.

\begin{tabular}{|l|r|r|r|r|}
\hline \multicolumn{1}{|c|}{ Question } & $\begin{array}{c}\text { Number of } \\
\text { Respondents }\end{array}$ & Mean & $\begin{array}{c}\text { Standard } \\
\text { Deviation }\end{array}$ & $\begin{array}{c}\text { Agree or } \\
\text { Strongly } \\
\text { Agree }\end{array}$ \\
\hline $\begin{array}{l}\text { I took on one or more tasks that I had not } \\
\text { done at work before }\end{array}$ & 37 & 3.76 & 1.116 & $73 \%$ \\
\hline $\begin{array}{l}\text { I learned something about my own abilities } \\
\text { and interests }\end{array}$ & 37 & 3.51 & 1.070 & $49 \%$ \\
\hline $\begin{array}{l}\text { I learned something about the abilities or } \\
\text { interests of my coworkers }\end{array}$ & 37 & 3.59 & 1.066 & $60 \%$ \\
\hline $\begin{array}{l}\text { I learned one or more ways my coworkers } \\
\text { and I can better help VR customers }\end{array}$ & 37 & 3.62 & 1.037 & $51 \%$ \\
\hline $\begin{array}{l}\text { I learned one or more ways individuals and } \\
\text { organizations outside VR (comm.. partners, } \\
\text { employers, etc.) can help VR customers }\end{array}$ & 37 & 3.84 & .764 & $68 \%$ \\
\hline
\end{tabular}

Table 5.2 - Closed-ended survey questions - Personal Mastery

The largely positive response to the first question (73\% Agree or Strongly Agree) in Table 5.2, above, suggests that the majority of SIA participants took on tasks during SIA that were not part of their regular work responsibilities. A majority of respondents to the third question said they learned something about the abilities or interests of their coworkers (60\% Agree or Strongly Agree). This is consistent with the fact that SIA 
created a situation where staff members were working together more closely and more frequently than was typical in their workplace. A majority of respondents to the fifth question said they learned one or more ways individuals and organizations outside VR can help VR customers (68\% Agree or Strongly Agree.) This is consistent with the fact that each of the three participating VR offices chose a project which required interaction with community partners.

In response to the second question, about half of respondents said they learned something about their own interests and abilities (49\% Agree or Strongly Agree). In response to the fourth question, a similar percentage of respondents said they learned one or more ways they and their coworkers can better help VR customers (51\% Agree or Strongly Agree.)

The moderate level of positive responses to these two questions may relate to two factors. The first factor is that staff had already been asked to closely examine their interests and abilities - and to consider how they use, or could use, their strengths to help VR clients - during the strengths training course they attended prior to the start of SIA. The second factor is that many staff made comments during SIA that strongly suggested they prided themselves on their professional expertise, and on the comprehensive and creative approaches they had developed to assist their clients. I've been doing this for years - I know how to move my clients forward. In short, they may not have felt they needed to educate themselves further about their interests and abilities, or about how to be effective in assisting clients. 


\section{Review.}

The staff participating in SIA were introduced to the concept of personal mastery during the strengths training course they attended prior to the start of SIA. They were asked by trainers to consider their personal strengths, and the value of utilizing those strengths in their workplace. At the beginning of SIA, staff reviewed their personal strengths, and were encouraged to keep those strengths in mind as they moved through the SIA experience.

There is evidence that, during SIA, both supervisors and staff increased their personal mastery by learning about their coworkers' strengths and working styles, and developing more productive ways to collaborate with them; learning about community partners, and ways in which they - and their agency - could work more effectively with those partners; and taking on specific tasks they had not done at work before, thereby increasing their confidence and expanding their expertise.

Personal mastery is closely related to the discipline of mental models. Staff's development of mental models during SIA is addressed below.

\section{Mental Models}

Mental models are deeply ingrained assumptions/generalizations that influence how one understands the world, behaves in the world, and believes the world works. They are the lenses through which individuals view their world (Senge, 2006).

Prior to SIA, all but one staff member participating in SIA attended an agencysponsored strengths training class. During the class, the trainers encouraged attendees to recognize and examine their strongly-held mental models, and share those models with 
others. They explained that exposing and "testing" one's assumptions in this way often leads to more creative thinking and an expanded worldview.

During SIA, mental models were not frequently addressed in group interactions. This may have been because sharing one's perspective with others in a group setting involves some risk of ridicule, or worse - a risk not willingly taken before an individual's trust in a group, or group process, is well established. It may also have been due to the fact that changes in perspective are often subtle and gradual internal processes which evolve over an extended period of time.

Nevertheless, there is evidence that, during SIA, some individuals changed their view of certain aspects of their work world. The changes most frequently observed and commented upon were changes in participants' personal outlook, or approach to their work; shifts in participants' view of intra-office interactions; and new insights into work processes.

\section{Personal outlook/approach.}

Speaking of their experience with SIA, some staff noted that participating in their office's project made them view their work role - and their options within that role - in a new way. I think [SIA] kind of pried open my thinking about how I do my job. Others reported a new awareness of their potential, and a new willingness to expand the scope of their work. This project has helped me see a side of myself that I did not know existed; I am now not as afraid to work in the community as I once have been.

Supervisors spoke of their internal thought processes during SIA, and the ways in which this changed their interactions with staff. I wanted to get in there with the 
Employer Committee a little bit and start directing, and I just chose not to, because I thought, "This is a project, let's see how well it goes." In some instances, their reflections resulted in movement away from a top-down style of supervision and toward a more team-directed style of working. I learned that I can take a step back and not say, "Just do it this way," but, "Remember, we need to do this." And it really works.

\section{View of office interactions.}

SIA offered some staff a new perspective on interactions in their workplace. Better understanding of the dynamics of this office. They were able to see the value in understanding, and taking full advantage of, their coworker's strengths. [SIA] made me think about our Strengths Profile for our team, and how I can ask someone for their input based on their individual strengths.

During the course of SIA, and in the online survey, many participants commented on the clear advantages of well-conceived group efforts. Breaking the group into individual teams based on their strengths helps in working toward, facilitating, and getting to an end result. Even in instances where individuals were frustrated by their office's approach to SIA, they made positive comments about the learning organization concept. The [SIA] idea was great and, with a more professional staff, would be very powerful.

\section{Insights into work processes.}

Participation in SIA led some staff to new insights about work processes. In some cases, they realized there are more - and more creative - ways to accomplish important tasks. [I learned] there are a variety of ways to introduce VR services to the public. In 
other cases, they became increasingly certain that VR needs to connect and collaborate with other community organizations in a more systematic fashion. I am now thoroughly convinced that [counselors] need to consistently engage in comprehensive recruitment activities on an ongoing basis to develop a "pipeline" of customers who have potential for employment.

Some of what staff discovered during SIA underscored the importance of VR communicating clearly and often with individuals and organizations in the community. Once the counselors were going out and talking to different people, they realized all the misconceptions people had about what we could offer...It was kind of, you know, an eye opener. Some staff insights led to contemplation, and new questions, rather than ready answers. I think that what I've gotten out of this is kind of a growing sense of we're not getting the right clients. These questions were often grist for animated discussions in SIA group meetings.

\section{Responses to closed-ended survey questions.}

The responses to two closed-ended online survey questions included in Table 5.3 below, are pertinent to the discussion of mental models.

\begin{tabular}{|l|c|c|c|c|}
\hline \multicolumn{1}{|c|}{ Question } & $\begin{array}{c}\text { Number of } \\
\text { Respondents }\end{array}$ & Mean & $\begin{array}{c}\text { Standard } \\
\text { Deviation }\end{array}$ & $\begin{array}{c}\text { Agree or } \\
\text { Strongly } \\
\text { Agree }\end{array}$ \\
\hline $\begin{array}{l}\text { I learned something about my own } \\
\text { abilities and interests }\end{array}$ & 37 & 3.51 & 1.070 & $49 \%$ \\
\hline $\begin{array}{l}\text { I learned something about the abilities or } \\
\text { interests of my coworkers }\end{array}$ & 37 & 3.59 & 1.066 & $60 \%$ \\
\hline
\end{tabular}

Table 5.3 - Closed-ended survey questions - Mental Models

Almost half of respondents indicated they learned something about their own abilities and interests during SIA (49\% Agree or Strongly Agree.) The positive responses 
to this question are consistent with comments from SIA participants who indicated they changed their perspective or approach to their work during SIA.

Six out of ten respondents indicated they learned something about the abilities or interests of their coworkers. The positive responses to this question are consistent with comments from SIA participants about positive interactions they had with, and new understandings they developed about, coworkers during their office's SIA project.

\section{Review.}

Staff participating in SIA were introduced to the concept of mental models during the strengths training course they attended prior to the start of SIA. They were encouraged by trainers to recognize and examine their strongly-held mental models, and to share those models with others.

Although mental models were infrequently addressed in group settings during SIA, there is evidence that some staff experienced changes during SIA in the way they view and approach their own work; in their view of interactions with coworkers; and in their perspective on work processes.

Building shared visions is another of the five learning organization disciplines. Staff's development of shared visions during SIA is addressed below.

\section{Building Shared Visions}

Building shared visions is the process of translating individual visions into a shared picture of the future that an organization, or a workgroup within an organization, wants to create. A true shared vision is a common caring about a goal shared by all 
members of a working group, and provides the focus and energy for subsequent team learning (Senge, 2006; Senge, et al., 1999).

In each of the three offices participating in SIA, staff met as a group to select a goal that would serve as the focus of their office's SIA project. In each office, staff worked together to select a short-term goal - sharing their ideas, information, and opinions - and ultimately reached consensus regarding a goal.

Observations and comments regarding the process of selecting a group goal varied widely. Some participants felt quite positive about the experience, while others were critical of the process. In each of the three offices, some staff began with reservations about, or resistance to, the concept of sharing personal visions to arrive at a common goal. However, the majority of participants gradually changed their attitude and behavior. They became more positive and engaged during the goal-setting process, or as they began to work toward the goal that was chosen.

\section{Positive responses.}

Some staff reported that the process of setting their office's goal went smoothly. We set the goal pretty readily up front. Others shared their appreciation of the process as it unfolded. It was kind of nice to observe kind of the flow and exchange of ideas, especially during the brainstorming phase in the beginning of the project.

Several staff remarked on positive effects of the goal-setting process. With VR freshly released from the constraints of the federal Order of Selection, staff were pleased to find themselves with the time and the opportunity to make plans and establish priorities for their workplace. I think [the goal-setting phase] reinvigorated our need to 
go out in the community, and kind of tap those agencies that somehow got neglected due to the [Order of Selection] waiting list.

\section{Negative responses.}

The majority of negative comments about goal-setting for SIA focused on the amount of time taken to select a goal. Some staff suggested more time should have been taken to assure that the goal was a good fit for staff participating in the process. I do think if we had taken longer, we probably could have found the goal that more people were interested in attacking, or that played to what more people felt were their strengths and

comfort zones. They felt the speed at which their office's goal was selected left some staff uncommitted to the goal. There wasn't sufficient time come up with an idea we all agreed with. I'm not sure if everyone bought into it.

A few staff held an opposing view. They believed their office spent too much time choosing an SIA goal. I realize it's important to brainstorm to arrive at a goal, but I just felt this process took too long.

\section{Shifting responses.}

Selecting the SIA goal was the first task set for each office participating in SIA. Thus, participants in the project were simultaneously holding down usual job responsibilities, familiarizing themselves with the concept of learning organizations, and being encouraged to actively engage in choosing a worthwhile and doable office goal that would make good use of staff strengths.

One supervisor spoke about recognizing and responding to the resistance of some staff to establishing ANY new goal. Everybody has a huge job with all these building 
caseloads. Layering another thing on them is how some people perceived [SIA]. For them to take ownership... they had to pick something they were interested in. Another supervisor described her staff's goal-setting journey, from an initially overambitious goal to one that was much more realistic. They started really big, and then tried to narrow it down to something that could actually get accomplished. I think that was another challenge...but it worked out really well.

Some staff's initial skepticism about their office's chosen goal diminished once the SIA project was underway. One staff member from Office $\mathrm{C}$ was pleasantly surprised by her change in outlook, and that of her coworkers. I really didn't think we'd chosen a good something to work on. But [the goal] not only became owned and valued by each individual office, but as a unit, which I didn't expect to happen.

\section{Responses to closed-ended survey questions.}

The response to one closed-ended online survey question included in Table 5.4, below, is pertinent to the discussion of shared visions.

\begin{tabular}{|l|c|c|c|c|}
\hline \multicolumn{1}{|c|}{ Question } & $\begin{array}{c}\text { Number of } \\
\text { Respondents }\end{array}$ & Mean & $\begin{array}{c}\text { Standard } \\
\text { Deviation }\end{array}$ & $\begin{array}{c}\text { Agree or } \\
\text { Strongly } \\
\text { Agree }\end{array}$ \\
\hline $\begin{array}{l}\text { The supervisor and staff in my office } \\
\text { worked together to select one short-term } \\
\text { goal for the project }\end{array}$ & 37 & 4.32 & .973 & $89 \%$ \\
\hline
\end{tabular}

The largely positive response to the question above (89\% Agree or Strongly Agree) indicates that the majority of SIA participants concurred that the supervisor and staff in their office engaged in a collaborative process to select the short-term goal for their office's SIA project. 


\section{Review.}

There is strong evidence that all staff members in each office participated in building a shared vision during SIA. All staff were present and part of the discussion and decision-making that led to selection of their office's SIA goal. However, the evidence that all staff members were strongly committed to the selected goal is not as strong.

Some staff reported that they and their coworkers had reservations about their office's chosen goal early in the process, but that their commitment to the goal increased as their office's SIA project evolved. This evolution was observed in each of the three participating offices, and was critical to the success of each of the three SIA projects.

The discipline that naturally follows an organization's or work group's development of a shared vision is team learning. The evolution of team learning during SIA is discussed below.

\section{Team Learning}

Team learning is a process in which coworkers in an organization "think together" until there is consensus about how to reach a particular goal. Team learning is vital because teams, not individuals, are the fundamental learning unit in modern organizations. Unless teams in an organization can learn, the organization cannot learn (Reason \& Bradbury, 2001; Senge, 2006).

During SIA, staff in each participating office met as a group to establish steps to reach their office's chosen short-term goal. After reaching consensus on the steps necessary, they worked together to determine what the timeline would be for each step, and which staff would take on which responsibilities. They then proceeded to work - as 
individuals, in small groups of coworkers, or with community partners - toward accomplishing the agreed-upon steps.

As each office's process evolved, staff met as a group on a regular basis to report on their progress, and to share their experiences, insights, and recommendations for next steps. All staff members attended each group meeting, unless they had made previous arrangements with their supervisor (regarding vacation, out-of-office trainings, etc.), or an emergency arose.

The types of team learning which presented during SIA, and were most often observed and commented upon, fell into two broad categories: staff working and learning together as a large group, or in smaller sub-groups; and staff working and learning in conjunction with community partners.

\section{Staff working and learning together.}

Many staff commented on the fact that, during SIA, their office "came together" and did what was necessary to move toward their office's chosen goal. The measure of success was everybody showing up at 6:45 in the morning and being upstairs in that room and, you know, we pulled it off! It was often noted that each individual in the office played some part in the success of the SIA project. Well, we accomplished our goal, and everybody had a role.

As the SIA project evolved in each of the three participating offices, it became clear that the teamwork taking place was, to some degree, a departure from the way business had previously been conducted. There was some teaming going on that hadn't existed prior to the project, and that was nice. Many staff commented that the structure 
of the project encouraged communication and coordination with coworkers. We practiced working as a team...we were able to communicate effectively. Most viewed this as a positive development for their office. I think it was a good team-building exercise. I really do think so. Some offered examples of ways in which teamwork during SIA led to important team learning. We pinpointed a specific way to help our office work smarter, and develop a better communication with other agencies.

\section{Staff working and learning with community partners.}

During SIA, staff interacted regularly not only with one another, but also with a variety of community partners. For the most part, they reported positive interactions with individuals and organizations outside of VR. I think that we've made better connections I have - with workers next door... as a result of the extra effort, there's a more positive outlook, both directions, more of a willingness to work together - to partner - for someone. In addition to improved relationships, staff indicated that both they and the community partners they connected with gained valuable information. I think there is a better understanding of what we do and what Agency $S$ does.

Some of the most important learning that took place during SIA was drawn from roadblocks encountered on the way to the offices' goals. In some cases, staff recognized that one aspect of the process, such as the timeframe, would need to be adjusted if a similar effort were attempted in the future. It takes a whole lot more investment and time in personal interactions than we counted on. In other cases, they realized that a number of changes would be necessary in order to reach their goal. We discovered the complexity 
of job placement skills, and the difficulty of establishing working relationships in the community.

During meetings and in survey comments, staff often spoke of the work done during SIA as a positive first step in a longer process they were envisioning. Provided an opportunity to set up needed communication and networking with a partner. Many made it clear that they anticipated long-term benefits from SIA. We can work together better with Agency S now that we know each other, and have a better understanding of how we each help customers.

\section{Responses to closed-ended survey questions.}

The responses to six closed-ended online survey questions included in Table 5.5, below, are pertinent to the discussion of team learning.

\begin{tabular}{|l|c|c|c|c|}
\hline \multicolumn{1}{|c|}{ Question } & $\begin{array}{c}\text { Number of } \\
\text { Respondents }\end{array}$ & Mean & $\begin{array}{c}\text { Standard } \\
\text { Deviation }\end{array}$ & $\begin{array}{c}\text { Agree or } \\
\text { Strongly } \\
\text { Agree }\end{array}$ \\
\hline $\begin{array}{l}\text { The supervisor and staff in my office each } \\
\text { agreed to do one or more things to help the } \\
\text { office reach the goal }\end{array}$ & 37 & 4.22 & 1.109 & $84 \%$ \\
\hline $\begin{array}{l}\text { The supervisor and staff in my office each did } \\
\text { one or more things to help the office reach the } \\
\text { goal }\end{array}$ & 37 & 4.19 & 1.151 & $81 \%$ \\
\hline $\begin{array}{l}\text { My supervisor, coworkers and I worked } \\
\text { together on one or more parts of the project }\end{array}$ & 37 & 3.83 & 1.056 & $80 \%$ \\
\hline $\begin{array}{l}\text { My supervisor, coworkers and I shared new } \\
\text { knowledge and insights }\end{array}$ & 37 & 3.68 & .973 & $62 \%$ \\
\hline $\begin{array}{l}\text { My supervisor, coworkers and I kept one } \\
\text { another informed of progress made on the } \\
\text { project }\end{array}$ & 37 & 3.95 & .941 & $78 \%$ \\
\hline $\begin{array}{l}\text { My supervisor, coworkers and I developed a } \\
\text { stronger working relationship with others }\end{array}$ & 37 & 3.59 & 1.013 & $52 \%$ \\
\hline
\end{tabular}

The strong positive responses to the first three questions above underscore that supervisors and staff worked as a team during SIA in an effort to reach their office's 
established goal. The responses to the fourth and fifth questions show that the majority of staff experienced ongoing in-office communication of information and insights throughout the SIA project.

The responses to the last question indicate that just over half of staff (52\% Agree or Strongly Agree) felt that they developed stronger working relationships with individuals or organizations outside VR during the SIA project. This is consistent with comments made concerning staff's interactions with community partners. Some comments described the development of very positive connections with partners over the course of SIA. Others comments suggested that efforts to connect to and work with outside individuals and agencies were fraught with some difficulties.

\section{Review.}

There is strong evidence that, as they moved through the SIA project, the majority of staff in each of the participating offices learned things of value and of use to their workgroup. In some cases, staff learned from one another; in other instances, they learned from their interactions with community partners.

Team learning is far more than an intellectual process. Being part of a successful team effort can foster pride, boost morale, increase motivation, and promote a sense of support and connectedness in the team members. Observations and staff comments suggest that many of the SIA participants experienced these benefits as a result of their team learning experience.

As in any attempt to work more closely with a group of individuals, SIA participants experienced disappointments, frustrations, and failures to successfully connect or collaborate with others while working on their office's SIA project. Despite 
the occasional setbacks, the benefits they accrued from the SIA experience appeared to far outweigh the costs.

The final learning organization discipline is systems thinking. The evidence of systems thinking that presented during SIA is discussed below.

\section{Systems Thinking}

Systems thinking is looking at the interrelated parts of a process, and recognizing and examining patterns and possibilities. In the context of learning organization theory, Senge (2006) refers to systems thinking as the fifth discipline. It is the discipline that integrates the other four disciplines - personal mastery, mental models, building shared visions, and team learning - into a coherent body of theory and practice. This is done by consistently looking at how each discipline is connected to the others.

During SIA, staff in each of the participating offices met regularly as a group to share information and insights about their project-related experiences, assess progress, and discuss and decide upon changes necessary to move the project forward. Between group meetings, they worked together with smaller groups of their coworkers to accomplish particular tasks, and with community partners who had a role and an investment in the office's project.

The developments reflecting systems thinking that were most often observed and commented upon were staff sharing information and ideas during SIA; staff's increasing awareness of personal strengths, the wisdom in making good use of each individual's strengths; and staff reporting value in the learning organization process. 


\section{Staff sharing information/ideas.}

As staff in each office worked toward their chosen SIA goal, there was ongoing communication about the different tasks involved in the office's process. Our office benefited from the continued meetings within our office - even if these meetings were very brief. We were able to communicate with each other new ideas, and others that have not worked. As the project evolved, staff's attention was increasingly focused on the individuals or agencies critical to the success of the project's goal. People were talking about Agency S more...you'd hear people say "Oh, yeah, we need to talk about that in our Agency S meeting." That was the most significant change, is that it was at the top of people's minds.

During SIA, conversations frequently centered on needed adjustments in the office's approach to their selected goal. It seems like there's constant change, and there probably will still be constant change. So, we've had to adjust what we want and what our expectations are in order to meet with Agency $Y$. Quite often, conversations that began as SIA-related discussions evolved into more general discussions about the way in which the office conducted everyday business. [SIA meetings] led to, I think, more indepth discussions about how [different staff] work differently, and how maybe we can improve things.

\section{Consideration of personal strengths.}

As participating staff completed a strengths training course shortly before SIA began, the concept of developing awareness of each individual's strengths - and using those strengths to advantage in the workplace - was fresh in their minds. Comments 
suggested that some staff entered the SIA project with an established belief in the value of recognizing staff strengths. I think there's a lot of value in having an understanding of the people in your office, what their strengths are, who to tap for different responsibilities. I always have.

Other staff came to recognize the benefits of understanding and relying on personal strengths during SIA. I was able to see each [staff member] has their own strengths, and how it's helpful to be aware of that...It was interesting to see how we could utilize our individual strengths and work together as a group. It was frequently pointed out that it took a project such as SIA to bring staff strengths to the fore. We could identify staff strengths more readily doing a project like this...Brought out the amazing strengths and abilities of this staff.

\section{Valuing the learning organization process.}

In addition to sharing ideas and recognizing personal strengths, staff reported a variety of benefits to participating in SIA. Some welcomed the chance to carefully consider the effectiveness of their office's current work processes. This whole project allows us to pause and think about how it really takes steps and teamwork to work with $V R$ customers in reaching their individual work goals. Others saw value in strengthening connections with partners outside VR. It was helpful to see how developing strong employer connections can be beneficial for VR as an agency, and for the community rehabilitation partners that we work with.

Many staff indicated that their office gained valuable insights during SIA. Now that we have done this once, I think we learned some important lessons on what worked 
and what didn't. They also indicated a desire to sustain and grow the gains their office made during the project. I'd suggest some kind of ongoing follow-up...something like making one contact a month or something. Not putting a huge burden on people, but helping them keep the good things we've accomplished going.

\section{Responses to closed-ended survey questions.}

The responses to two closed-ended online survey questions included in Table 5.6, below, are pertinent to the discussion of systems thinking.

\begin{tabular}{|l|c|c|c|c|}
\hline \multicolumn{1}{|c|}{ Question } & $\begin{array}{c}\text { Number of } \\
\text { Respondents }\end{array}$ & Mean & $\begin{array}{c}\text { Standard } \\
\text { Deviation }\end{array}$ & $\begin{array}{c}\text { Agree or } \\
\text { Strongly Agree }\end{array}$ \\
\hline $\begin{array}{l}\text { My supervisor, coworkers and I shared } \\
\text { new knowledge and insights }\end{array}$ & 37 & 3.68 & .973 & $62 \%$ \\
\hline $\begin{array}{l}\text { My supervisor, coworkers and I kept one } \\
\text { another informed of progress made on } \\
\text { the project 78\% }\end{array}$ & 37 & 3.95 & .941 & $78 \%$ \\
\hline
\end{tabular}

The majority of staff reported that they shared new knowledge and insights with their supervisor and coworkers during SIA (62\% Agree or Strongly Agree.) This is consistent with observations of SIA meetings and other office interactions during SIA, and with responses to open-ended survey questions.

A somewhat larger percentage of respondents reported they kept their supervisor and coworkers informed during SIA (78\% Agree or Strongly Agree.) This fits with observations of large-group and small-group SIA discussions, during which updates were elicited from each staff member present. It also fits with comments made in response to open-ended survey questions.

\section{Review.}

There is evidence that, during SIA, both supervisors and staff engaged in systems thinking. This was reflected in their ongoing sharing of information and ideas with one 
another throughout the SIA project; the fact that they recognized and utilized the personal strengths of their coworkers; and their comments about SIA - and the learning organization process central to SIA - being of value to them and their workgroup.

\section{Research Question 2: What factors facilitated the implementation of the modified learning organization model?}

\section{Storyline for Research Question 2}

The factors that were most powerful in facilitating the adoption of the modified learning organization model were: (a) the structure of the Strengths in Action (SIA) project; (b) supervisor support; (c) growing connections among staff; (d) growing connections between staff and community partners; and (e) staff's increasing ownership of the SIA project.

Figure 5.3 - Storyline for Research Question 2

\section{Data Sources}

The four data sources analyzed to answer Research Question 2 were observations, interviews, online survey open-ended questions, and online survey closed-ended questions. Table 5.7, below, summarizes which data sources contained evidence for which of the facilitating factors.

\begin{tabular}{|l|c|c|c|c|}
\hline & Observations & Interviews & $\begin{array}{l}\text { Online Survey } \\
\text { Open-ended } \\
\text { Questions }\end{array}$ & $\begin{array}{l}\text { Online Survey } \\
\text { Closed-ended } \\
\text { Questions }\end{array}$ \\
\hline Structure of SIA Project & $\mathrm{X}$ & $\mathrm{X}$ & $\mathrm{X}$ & $\mathrm{X}$ \\
\hline Supervisor Support & $\mathrm{X}$ & $\mathrm{X}$ & $\mathrm{X}$ & $\mathrm{X}$ \\
\hline $\begin{array}{l}\text { Growing Connections } \\
\text { Among Staff }\end{array}$ & $\mathrm{X}$ & $\mathrm{X}$ & $\mathrm{X}$ & $\mathrm{X}$ \\
\hline $\begin{array}{l}\text { Growing Connections } \\
\text { Between Staff \& Comm. } \\
\text { Partners }\end{array}$ & $\mathrm{X}$ & $\mathrm{X}$ & $\mathrm{X}$ & \\
\hline $\begin{array}{l}\text { Staff's Increasing } \\
\text { Ownership of SIA }\end{array}$ & $\mathrm{X}$ & & & \\
\hline
\end{tabular}

Table 5.7 - Summary of data sources - Research Question 2 


\section{Overview}

The remainder of this section of Chapter 5 is a detailed discussion of the factors which facilitated the implementation of the modified learning organization model: the structure of the SIA project; supervisor support; growing connections among staff; growing connections between staff and community partners; and staff's increasing ownership of SIA. A comprehensive outline of the discussion about Research Question 2 findings is provided in Figure 5.2 below. 
Structure of SIA Project

- Creating time and space

- Program manager and researcher contributions

- Accountability

- Utilization of personal strengths

- Responses to closed-ended survey questions

- Review

Supervisor Support

- Types of support

- Supervisors' observations

- Responses to closed-ended survey questions

- Review

Growing Connections Among Staff

- Bringing staff together

- Staff supporting each other

- Improved communication

- Camaraderie

- New relationships built

- Responses to closed-ended survey questions

- Review

Growing Connections between Staff and Community Partners

- Connections forged with community partners

- Teaching, or learning from, community partners

- Long-term benefits/sustainability

- Responses to closed-ended survey questions

- Review

Staff's Increasing Ownership of SIA

- Supervisor's support of staff ownership

- Staff assuming responsibilities

- Staff ownership increasing over time

- Review

Figure 5.4 - Outline of discussion of Research Question 2 Findings

\section{Structure of the SIA Project}

The manner in which the SIA project was structured served to "set the stage" in each of the three participating offices so staff could work together effectively to select, and then reach, a goal. Throughout the project, supervisors and staff in each of the offices made it clear they saw value in having an established framework within which to pursue their chosen goal. It provided a lot of structure. And I think that was probably to me the 
most valuable thing about it. The structure. The way in which SIA was organized helped staff to maintain focus on their chosen goal. We'd had this idea we were going to do outreach, but it wasn't really rolling and happening - other things kept taking our time. But once, you know, we formalized it, that's what really got it off the ground.

The four aspects of the SIA project structure most often observed and commented upon were: the creation of time and space to pursue a goal; the contributions of the VR Program Manager and researcher; the way in which participants were held accountable; and the utilization of staff members' individual strengths to move the project forward.

\section{Creating time and space.}

In agreeing to participate in the SIA project, the supervisor and staff in each of the three participating offices agreed to carve out time and space over the course of four to six months to work on a project of their choice, using a collaborative model that was not standard practice for their group. In effect, for a period of several months, the three participating offices gave the SIA project they selected priority over other tasks they were already working on, preparing to work on, or contemplating.

The supervisor and staff in each office agreed at the beginning of the SIA project to meet as a whole at specific times to conduct SIA-related business. In all three offices, they chose to conduct "SIA business" as a defined segment of their standard staff meetings, in order to assure the presence of the majority of the staff. In addition, staff met in small groups with coworkers or community partners between the large group meetings. This played out differently in each office, as each office's chosen project required different interactions both inside and outside the office. 
As the project in each office got underway, staff began to recognize the benefits of a committing time to a clearly defined group effort. They realized that by structuring it, and taking the time to talk about it, and then having deadlines... it put more structure to what they were doing. And they saw value in it. They realized that focusing the entire office's attention on one effort could lead to gains that were otherwise unattainable. If we didn't make this a priority, we were never going to get out of the vicious circle of just getting a bunch of cases dumped into the office that really have very little rehabilitation potential.

Some staff reported that the SIA project encouraged them to do something that was, for them, completely out of the ordinary. Pushed me to do tasks that I typically do not make time for. Others appreciated the chance to use well-honed skills that were not

often used in their daily work. While I have a lot of experience with community outreach, I have been very busy in the office and did not do much of it before [SIA] in seeking out referrals. I focused on the need to find new sources of referrals in order to meet our goals. Still others were pleased to find time to work on projects that had been "waiting in the wings" for too long. I was able to dedicate time to focus on things we had been planning to do.

\section{Program manager and researcher contributions.}

As outlined in Chapter 3 (Methods), at the beginning of the SIA project in each participating office, the Program Manager and I introduced ourselves and outlined the concept of the SIA project, first to the office supervisor and then to the office's entire 
staff. I was present at all the group meetings in each of the three offices during SIA; the Program Manager attended the majority of the meetings.

During the project, the Program Manager functioned as an open-ended support to the supervisor/facilitator, playing a slightly different role in each of the three offices. The Program Manager's responsibilities included explaining how the project chosen by the office intersected with efforts at VR headquarters; helping to define the tasks to be accomplished in each meeting; assisting the supervisor during discussions by writing down key points as they were made; obtaining supplies and materials requested by the offices; and, in one instance, filling in as meeting facilitator when the supervisor was called away.

I provided written agendas for each meeting, to assist in framing and focusing the group discussions. The agendas outlined such tasks as deciding when to meet to conduct "SIA business," selecting a goal, and determining roles for each project participant. As the process moved forward, the agendas reflected the progress the office had made to date, and logical "next steps." In addition, at the request of a supervisor or staff member, I occasionally generated specific lists or summaries that grew out of staff discussions.

Both the Program Manager and I were routinely thanked by the participating supervisors and staff for participating in the SIA meetings. A staff member approached me after one of the first meetings and said that having "new blood" at the office's staff meetings was very energizing. One supervisor made it very clear that our willingness to be involved to the extent that we were was the only reason she agreed to participate in the project. If it had not been for you and [Program Manager] providing the structure, and 
the guidance, and the note-keeping, and the agendas that you prepared, I wouldn't have done this.

Some staff indicated that having outsiders come to the office to participate in the SIA project lent importance to the project in their mind. Knowing that you and [Program Manager] were coming really created a greater expectation than just that you have to report to [supervisor] on Tuesday when we talk. It gave the activity a lot more weight, it made it more official. Some praised specific contributions made. [Program Manager] gave us a format, she gave us structure to follow, as far as putting all of our ideas together, and she kept us on track on what our overall goal or mission was to do. Overall, comments indicated that our contributions were considered to be of value. If you two weren't there, we still would have been talking about "Go out and make contacts in the community" and "Tell me what you've done." But it wouldn't have been as well thought out, and not as structured.

\section{Accountability.}

The project format made participants accountable for their part in the project, both as individuals and as a group. First, the entire staff was involved in selecting a goal, which created group accountability. Once the goal was established, each individual was asked to commit to making some contribution toward the chosen goal, which fostered individual accountability. The standard of accountability continued as each individual (or, in some instances, each small group) was asked to report on their progress in group meetings throughout the SIA project. 
In all three participating offices, participants spoke positively about the accountability inherent in the SIA project structure. People were being held accountable, because they were coming back and reporting at the staff meetings on their progress, and knew they would be...that was the most valuable thing, in my opinion. Some saw clearly structured projects such as SIA as the only way to keep staff "on task" with any project during a period of increasing workloads and constant changes. If we didn't have regular reminders in place that we have to do something, it would just fall by the wayside, because of all the other "crises" that constantly come up around here. One staff suggested SIA should continue in order to maintain accountability. This project held us accountable; it would be nice if it could be an ongoing project so the accountability was there.

\section{Utilization of personal strengths.}

As previously discussed, all but one participant in the SIA project had completed an agency-sponsored strengths training course prior to the start of SIA. VR management's support of the SIA project was tied to the belief that it would build on the concept of using personal strengths in the workplace. At the beginning of SIA, staff reviewed their five top individual strengths (as determined in their training) and their office's 'strengths profile.' During SIA, they were encouraged to focus on, develop, and use their own strengths, and to examine and use their coworkers' strengths. They were also encouraged to take opportunities to work outside of their normal job duties, in order to learn new skills and develop new perspectives. 
In early SIA group meetings, when staff were determining which roles different individuals should play in their chosen project, it was evident they were taking the personal strengths of staff members into account. They frequently called on certain individuals to step forward and use well-recognized skills and expertise. I think "Donna" should do that. You will, won't you? You're really good at that. Do you guys remember what a beautiful job she did on the [event] last year? As the projects moved forward, staff became increasingly aware of the value of making effective use of their own strengths and those of their coworkers. It allowed me to see how breaking the group into individual teams based on their strengths helps in working toward, facilitating, and getting to an end result.

The SIA project served to highlight the existing strengths of staff in each office. It made us look at individual strengths, and how we complement/help each other with strengths. In some instances, staff developed new strengths and new behaviors during the course of the SIA project. "Joanne" and "Louise" did a great job on graphics for the invitations and RSVPs, and really worked together. That was new territory for both of them. The majority of participants indicated that staff recognized and used their own strengths and those of others during SIA, and they found value in that process. Ifeel like it's just taught me, although I already know this, that if you play to people's strengths things are going to go better.

\section{Responses to closed-ended survey questions.}

The responses to three closed-ended online survey questions included in Table 5.8, below, are pertinent to the discussion of the structure of the SIA project. 


\begin{tabular}{|l|c|c|c|c|}
\hline \multicolumn{1}{|c|}{ Question } & $\begin{array}{c}\text { Number of } \\
\text { Respondents }\end{array}$ & Mean & $\begin{array}{c}\text { Standard } \\
\text { Deviation }\end{array}$ & $\begin{array}{c}\text { Agree or } \\
\text { Strongly } \\
\text { Agree }\end{array}$ \\
\hline $\begin{array}{l}\text { The supervisor and staff in my office } \\
\text { worked together to select one short-term } \\
\text { goal for the project }\end{array}$ & 37 & 3.83 & 1.056 & $89 \%$ \\
\hline $\begin{array}{l}\text { The supervisor and staff in my office each } \\
\text { agreed to do one or more things to help the } \\
\text { office reach the goal }\end{array}$ & 37 & 4.22 & 1.109 & $84 \%$ \\
\hline $\begin{array}{l}\text { The supervisor and staff in my office each } \\
\text { did one or more things to help the office } \\
\text { reach the goal }\end{array}$ & 37 & 4.19 & 1.151 & $81 \%$ \\
\hline
\end{tabular}

Table 5.8 - Closed-ended survey questions - Structure of SIA Project

The strong positive responses to the three questions suggest that the structure of the SIA project was in place in the participating offices, and that it served as a framework within which staff operated during the project. The responses establish that a large majority of SIA participants concurred that everyone in their office was involved in choosing the office's SIA goal; that each person in the office agreed to do something to further the chosen goal; and that everyone accomplished what they had agreed to do.

\section{Review.}

The structure of the SIA project was a factor which facilitated the implementation of the modified learning organization model. The supervisors and staff participating in the SIA project felt the structure of the project assisted them in their attempts to reach their chosen goals. This assistance came in several forms: time and space to pursue their goals; two "outside" parties willing to support their efforts; the expectation of accountability for all SIA participants; and strong focus on best use of staff's personal strengths throughout the project.

The support of VR supervisors was another facilitating factor. The nature of supervisors' contributions to the SIA project is outlined below. 


\section{Supervisor Support}

The support of VR supervisors was vital to the implementation of the SIA project. Without each supervisor's early support of the project, the Program Manager and I would not have been granted access to their office's staff to explain SIA and propose their participation in the project. Once SIA was underway, it was evident that the attitude and actions of the supervisor - who functioned in each office as the facilitator of the SIA group meetings, as the on-site "overseer" of SIA activities between meetings, and as a team member in project subgroups - set the tone for the office's SIA experience.

\section{Types of support.}

During SIA, the supervisor in each participating office was observed to be supporting the project in a variety of different ways. These included specific actions such as arranging time and space for SIA meetings; facilitating - and actively participating in SIA discussions and decision-making; encouraging staff to voice their ideas, experiences, and perspectives; publicly acknowledging the strengths and contributions of individuals and small work groups; volunteering to complete one or more SIA-related tasks; and sharing information with staff about trends or forces likely to positively or negatively impact the office's chosen project.

Supervisor support for SIA also presented in more global ways, such as a supervisor regularly making statements and taking actions that indicated investment in the office's project, and showing an ongoing willingness to "jump in" to help staff in practical ways. Without the amazing leadership of [Supervisor A], it probably would not 
have been so successful! She encouraged us all the way from beginning to end, and she was as involved as the rest of the staff.

Although the supervisor played a key role in the SIA project in each of the three participating offices, the specific contributions each supervisor made were rarely noted by staff. This may have been because staff assumed their supervisor would take the lead in the project, based on their supervisor's role in previous office projects. However, in SIA meetings, in interviews, and in survey comments, staff frequently complimented their supervisor in more general terms. In [Office C], we have a good supervisor, who is supportive of connections [with other agencies]. Staff made the point, in a variety of ways, that their supervisor was committed to whatever was best for the office. I think [Supervisor B] is open to anything that improves this office's functioning.

\section{Supervisors' observations.}

The supervisors themselves had much to say about their SIA experience. Some of their comments were very positive in nature. Everybody worked very hard...I was proud that we accomplished it, period. Others comments suggested that they sometimes felt pressured by their responsibilities in the SIA project. I was a nervous wreck a few days before, just making sure that all the details came together. Because I really felt like I was kind of the hub of the wheel.

One topic that often arose in conversation with the supervisors was their efforts throughout SIA to define their optimum role in the project. On the one hand, once they agreed to participate in SIA, they wanted to be truly engaged in the project and support their staff as needed. I felt like [staff] kind of did need me to dip in places. On the other 
hand, they wanted to be certain they gave their staff sufficient "voice" as the project evolved. I was able to step back, and let people talk about [SIA issues] and decide, and that really worked...I wanted it to be what they wanted, and not just what I wanted.

During SIA, each of the supervisors handled the tension between guiding the office's project and granting their staff voice in the project somewhat differently. In the end, they all agreed that striking a balance between the two roles was key to the project's success.

\section{Responses to closed-ended survey questions.}

The responses to five closed-ended online survey questions included in Table 5.9, below, are pertinent to the discussion of supervisor support.

\begin{tabular}{|l|c|c|c|c|}
\hline \multicolumn{1}{|c|}{ Question } & $\begin{array}{c}\text { Number of } \\
\text { Respondents }\end{array}$ & Mean & $\begin{array}{c}\text { Standard } \\
\text { Deviation }\end{array}$ & $\begin{array}{c}\text { Agree or } \\
\text { Strongly } \\
\text { Agree }\end{array}$ \\
\hline $\begin{array}{l}\text { The supervisor and staff in my office } \\
\text { worked together to select one short-term } \\
\text { goal for the project }\end{array}$ & 37 & 4.32 & .973 & $89 \%$ \\
\hline $\begin{array}{l}\text { The supervisor and staff in my office each } \\
\text { agreed to do one or more things to help the } \\
\text { office reach the goal }\end{array}$ & 37 & 4.22 & 1.109 & $84 \%$ \\
\hline $\begin{array}{l}\text { The supervisor and staff in my office each } \\
\text { did one or more things to help the office } \\
\text { reach the goal }\end{array}$ & 37 & 4.19 & 1.151 & $81 \%$ \\
\hline $\begin{array}{l}\text { My supervisor, coworkers and I worked } \\
\text { together on one or more parts of the } \\
\text { project }\end{array}$ & 37 & 3.83 & 1.056 & $80 \%$ \\
\hline $\begin{array}{l}\text { My supervisor, coworkers and I kept one } \\
\text { another informed of progress made on the } \\
\text { project }\end{array}$ & 37 & 3.95 & .941 & $78 \%$ \\
\hline
\end{tabular}

Table 5.9 - Closed-ended survey questions - Supervisor Support

The strong positive responses to the five questions indicate that the supervisors were an integral part of discussions, decision-making, and project-related activities throughout SIA. 


\section{Review.}

Supervisor support was a factor which facilitated the implementation of the modified learning organization model. Supervisors' support was evidenced in a variety of ways, including early support of the SIA project; facilitation of group meetings during SIA; assistance in completing SIA tasks; and encouragement and recognition of staff efforts.

Staff acknowledged and appreciated their supervisor's support during SIA. However, most of their comments on this topic were general rather than specific in nature. This may have been because they were conditioned to having their supervisor take the lead in office-wide projects. It may also have been because the collaborative nature of SIA increased the focus on group interactions and lessened the focus on any one group member.

The supervisors themselves spoke in detail about their approach to supporting staff during their office's SIA project. They wanted to be actively involved, but not so much so that they stifled their staff's engagement in and commitment to the project. Their comments suggest that finding the correct balance was not always easy for them, and that they encountered both stresses and successes in the SIA experience.

Growing connections among staff was another facilitating factor. The ways in which staff connections contributed to the success of SIA projects are examined below.

\section{Growing Connections Among Staff}

During SIA, individuals in the three participating offices spent more time working together in large and small groups than was usual in their work setting. The experience of 
working together on a common task had the effect of strengthening the connections among staff as a whole. In some instances, it also served to foster stronger relationships between particular staff members.

The five aspects of growing connections among staff most often observed and commented upon were: the intrinsic value of bringing staff together; the support that staff offered to one another; the improved communication among staff; the camaraderie that developed among staff; and the new relationships that were built.

\section{Bringing staff together.}

Day-to-day in VR, each counselor on staff carries and tends to his or her own client caseload. Support staff are either responsible for specific duties, or are assigned to assist particular counselors. This system does not require, or encourage, regular interactions among all staff members.

Many staff pointed out that SIA offered staff an opportunity to connect in a way that was out of the ordinary. The work can be isolating sometimes. [SIA] definitely brought us together. Some conveyed their belief that the opportunity to work together as a group was of more importance than the specific tasks that were accomplished. I thought it was of value to put everybody working together, in general...it's all about building relationships.

\section{Staff supporting each other.}

Throughout SIA, staff regularly supported one another by carving out time for project meetings and activities, sharing ideas and information, and assisting in a variety of ways with the completion of necessary tasks. Everyone supported one another, and we 
got it done. This support was extended to, and appreciated by, the supervisors involved in the project. Ifelt very supported by [my staff] in the whole process.

Even individuals who consider their workgroup to be a normally high-functioning team reported positive developments during SIA. I think our unit does really well, overall, with teaming, because if they know there's a need they'll offer assistance, always. But in this case, it really helped them team, more so.

\section{Improved communication.}

Some staff started SIA with the belief that if staff met regularly it would lead to improved communication. It is always good to bring all staff to work on a project $-a$ great way to open communication. During SIA meetings, it was frequently noted that working on the office's project was, in fact, a boon to staff communication. It's good to get together and talk like this.

A number of staff commented that the process of sharing and considering individual's ideas during SIA ideas brought staff closer together. It was fun to hear different people's ideas...It definitely brought us together. In some cases, the most valuable and productive communication occurred in "sub-groups" working together during SIA. As this was a two-member team, we were able to communicate effectively, and were able to "see" the benefit of networking with a partner for ourselves and our customers.

\section{Camaraderie.}

A number of staff commented on the sense of satisfaction they derived from working with coworkers to attain a common goal. It's fun to kind of team with other 
people and get some measure of success. Both supervisors and staff noted that participating in SIA helped some staff move past prior difficulties. We've had our ups and downs over the years I've supervised...factions within the group, stuff like that...I think they're pretty proud that we could pull it off, and they should be.

One supervisor felt that a key benefit of the SIA project was that it allowed staff to step away from their daily work and focus on the agency's mission, and the contributions they were making toward that mission. Taking a moment to say, "Wow, this is a great mission we have as an agency...we're part of something that matters. " It helped them to really feel more cohesive...everybody felt like they had a part in it. A number of staff made the point that working on the SIA project in conjunction with their coworkers lightened the experience considerably. We did it together, and we remembered to laugh.

\section{New relationships built.}

The regular staff interactions during SIA resulted in the formation of some new connections between coworkers. There were some bridges built. Because there tend to be little cliques, and some of the way the teams played out, I think there were interesting relationships built. Staff worked together in configurations that represented a shift from the office's norm. The [Rehabilitation Technicians] wanted to participate, wanted a piece of it, and actually took on pieces of it. That was a little surprising...I think that was really neat.

Conversations during SIA and survey comments made clear that relationships between some coworkers were formed - or deepened - during SIA. Some staff shared 
their observations of their coworkers' interactions. "Jolene" had to come out of her cubicle and go with "Margo," and they had to follow up with employers, so I saw that as a real powerful new working relationship between the two of them. Other staff spoke of specific changes in their own relationships with coworkers. I think it [SIA] did strengthen some relationships. I think it strengthened some of my relationships...I think [name deleted]'s finally realized that I'm not a threatening person.

\section{Responses to closed-ended survey questions.}

The responses to six closed-ended online survey questions included in Table 5.10 below, are pertinent to the growing connections among staff during SIA.

\begin{tabular}{|l|c|c|c|c|}
\hline \multicolumn{1}{|c|}{ Question } & $\begin{array}{c}\text { Number of } \\
\text { Respondents }\end{array}$ & Mean & $\begin{array}{c}\text { Standard } \\
\text { Deviation }\end{array}$ & $\begin{array}{c}\text { Agree or } \\
\text { Strongly } \\
\text { Agree }\end{array}$ \\
\hline $\begin{array}{l}\text { The supervisor and staff in my office worked } \\
\text { together to select one short-term goal for the } \\
\text { project }\end{array}$ & 37 & 4.32 & .973 & $89 \%$ \\
\hline $\begin{array}{l}\text { I learned something about the abilities and/or } \\
\text { interests of one or more of my coworkers }\end{array}$ & 37 & 3.59 & 1.066 & $60 \%$ \\
\hline $\begin{array}{l}\text { My supervisor, coworkers and I shared new } \\
\text { knowledge and insights }\end{array}$ & 37 & 3.68 & .973 & $62 \%$ \\
\hline $\begin{array}{l}\text { My supervisor, coworkers and I worked } \\
\text { together on one or more parts of the project }\end{array}$ & 37 & 3.83 & 1.056 & $80 \%$ \\
\hline $\begin{array}{l}\text { My supervisor, coworkers and I kept one } \\
\text { another informed of progress made on the } \\
\text { project }\end{array}$ & 37 & 3.95 & .941 & $78 \%$ \\
\hline $\begin{array}{l}\text { My supervisor, coworkers and I became a } \\
\text { stronger team }\end{array}$ & 37 & 3.38 & .924 & $43 \%$ \\
\hline
\end{tabular}

Table 5.10 - Closed-ended survey questions - Staff-to-Staff Connections

The positive responses to the first five questions (between 60\% and 89\% Agree or Strongly Agree) point to the ongoing interactions among staff during SIA, which appear to have strengthened connections between some staff members. The weaker response to the sixth and final question about becoming a stronger team (43\% Agree or Strongly 
Agree) may suggest that a majority SIA participants did not feel SIA strengthened their connections with coworkers; felt their improved connections with certain coworkers did not extend to their office as a whole; felt the connections made during SIA would not be sustained once the project was completed; or felt that they and their coworkers were already a strong team before SIA began. However, as outlined above, qualitative data told a different and more positive story about the impact of SIA on supervisor and coworker connections.

\section{Review.}

Growing connections among staff was a factor which facilitated the implementation of the learning organization model. SIA participants often pointed to new, or stronger, relationships developed within their office as a positive aspect of their involvement in SIA.

Specific areas that were observed and commented upon were the opportunities SIA afforded staff to work as a group, and the importance of those opportunities; the support that coworkers offered to one another while working on their office's SIA project; the improvements in communication within participating offices during SIA; the camaraderie that developed among staff while they were working to meet their SIA goal; and the new relationships that were built between some SIA participants.

Growing connections between staff and community partners was another facilitating factor. The connections that developed between staff and a variety of individuals and organizations in the community during SIA, and the ways in which these connections moved SIA projects forward, are described below. 


\section{Growing Connections Between Staff and Community Partners}

During SIA, each of the three participating offices selected a goal that required staff to contact individuals or organizations in the community where the office was located. The purpose of the contacts was to establish new relationships, re-establish lapsed relationships, or strengthen existing relationships with specific community partners.

Interactions initiated by staff during SIA took a variety of forms. These included: VR employees telephoning or e-mailing employees of other organizations; a VR employee meeting with a representative of another organization; a VR employee meeting with small groups of staff from another organization; and all staff in a VR office meeting with all staff from the office of another organization.

The three aspects of growing connections between staff and community partners most often observed and commented upon were: connections forged between staff and community partners; staff teaching, or learning from, community partners; and the promise of long-term benefits from connecting with community partners.

\section{Connections forged with community partners.}

During SIA, a good deal of effort was spent by staff in each of the participating offices to contact and establish connections with community partners. In some instances, connections were made with individuals and organizations that had not existed prior to SIA. Agency T, that's an incredible new connection...the potential for employment there is pretty intense, I think, they have a lot of jobs in childcare...So, that was a good one. In other cases, SIA afforded staff a chance to reconnect with partners they had worked with 
in the past. It allowed for the opportunity to finally start the reconnection of relationships that have been dormant for a while.

However, most often, the interactions that occurred during SIA served to strengthen existing, ongoing relationships with community partners. SIA gave VR staff the opportunity to meet with staff in other organizations in their community more frequently, and in new ways. We asked to meet with their whole staff, which we hadn't done before, and actually made a concerted effort to improve relationships.

The increased interaction with community partners led to a variety of positive developments. Staff often noted that the quality of communication between VR and specific partners had improved. We've been able to develop better and more frequent ways to communicate with Agency S. They also noted positive changes in the tone of their interactions with staff from other organizations. I saw quite a bit of change in attitude, plus I felt, even seeing some of them in the hall, I felt like there was a lot more back and forth, more positive stuff going on. At times, these encouraging changes extended to individuals and organizations that had previously presented difficulties for VR staff. I can't say that we completely eliminated the us/them mentality with Agency Y, but I think we made a lot of strides...I've seen a lot of progress.

\section{Teaching, or learning from, community partners.}

During SIA, it was obvious that many staff took pride and satisfaction in the work they did to educate other organizations about VR practices. I liked being able to reach out into the community, and let them know about VR and the services we provide. Some reported that their efforts to share information were beginning to reap practical rewards. 
Now that we've gone through SIA, and we can explain better to Agency $S$ what an appropriate referral is, it has kind of stopped all the unnecessary back and forth, people coming in and out for no reason.

Some comments focused not on what VR staff had taught others during SIA, but rather on what they had learned from others. We're beginning to understand the requirements that our customers have with Agency $P$. This helps VR provide more appropriate services. However, the majority of comments on this topic suggested that valuable learning took place on both sides when VR staff engaged community partners during SIA. Agency S had questions, or they thought we did things a certain way, but when we talked about it, I think there was a better understanding of what we do and what they do.

\section{Long-term benefits/sustainability.}

Staff in each of the three participating offices made it clear from the beginning of their SIA project that they hoped, and intended, that the work they did during SIA would lead to long-term improvements in their interactions with community partners. They anticipated that stronger connections with community partners would have a variety of benefits for their clients.

As the offices' SIA projects evolved, observations and comments suggested that staff's efforts to build relationships with community partners were beginning to pay off. The word is getting out in the community; we are being able to reach more people. They saw evidence that the connections they were building would help their existing clients. $V R$ customers will benefit from stronger partnerships [with community partners]. They 
also reported that SIA had helped them to access new clients. The project helped in reaching out to some customers who would otherwise not have known about VR services.

Some staff expressed confidence that gains made during SIA would be sustained after their office's SIA project was completed. Now we've broken the ice. And we've got the ball in motion. I don't see [connections made with community partners] just dropping off everybody's radar. This is going to continue. Others thought beyond the gains made during SIA, and suggested there would be value in expanding the scope of their office's project. I think [SIA] was great. The only way I could see it improved is to expand the framework to other community partners.

\section{Responses to closed-ended survey questions.}

The responses to the one closed-ended online survey question included in Table 5.11 , below, are pertinent to the growing connections between staff and community partners during SIA.

\begin{tabular}{|l|c|c|c|c|}
\hline \multicolumn{1}{|c|}{ Question } & $\begin{array}{c}\text { Number of } \\
\text { Respondents }\end{array}$ & Mean & $\begin{array}{c}\text { Standard } \\
\text { Deviation }\end{array}$ & $\begin{array}{c}\text { Agree or } \\
\text { Strongly Agree }\end{array}$ \\
\hline $\begin{array}{l}\text { My supervisor, coworkers and I developed } \\
\text { a stronger working relationship with others } \\
\text { (VR customers, community partners, } \\
\text { employers, etc.) }\end{array}$ & 37 & 3.59 & 1.013 & $52 \%$ \\
\hline \multicolumn{2}{|l|}{ Table 5.11 - Closed-ended survey questions - Staff-to-Community Partner Connections } \\
\hline
\end{tabular}

Just over half of the respondents (52\% Agree or Strongly Agree) reported that their office staff developed stronger working relationships with others during the course of the office's SIA project. Those that responded less positively may have been among those who commented that, although connections made during SIA were good 
beginnings, more time than the SIA project allowed was needed to establish strong relationships with the community partners contacted during SIA.

\section{Review.}

Growing connections between staff and community partners was a factor which facilitated the implementation of the learning organization model. During SIA, each of the three participating offices selected outreach to a particular category of community partners as their office's goal. Therefore, the development of connections with those community partners was the primary purpose of their SIA project.

Observations and comments made during SIA confirmed that the participating offices had some success in establishing connections with community partners. In some instances, new connections were made; in others, established connections were deepened and strengthened. Participating staff reported finding satisfaction and value in teaching community partners about their work, and in learning more about the work of others. Some expressed confidence that the connections made with community partners during SIA would be maintained after the project's completion; others suggested that their office should continue their efforts and expand the scope of their office's project.

Staff's increasing ownership of SIA was another facilitating factor. The ways in which staff's engagement with their office's SIA project contributed to the project's success are examined below.

\section{Staff's Increasing Ownership of SIA}

At the beginning of SIA, the majority of participants were not strongly engaged in or committed to their office's chosen SIA project. Early in each office's process, some 
staff viewed SIA as a burden forced upon them by their supervisor or agency managers; some staff believed the SIA project would take time away from more important work tasks; some staff were unenthusiastic about the specific project their office chose to work on; and some staff feared they, and a few trusted coworkers, would end up doing the majority of work on the project.

As the project in each office evolved, many staff developed more connection to, and a stronger sense of ownership in, their office's SIA project. The indicators of increasing ownership most often observed and commented upon were: supervisors' belief in the value of staff ownership; staff assuming project-related responsibilities; and staff ownership increasing over the course of the SIA project.

\section{Supervisors' support of staff ownership.}

In each of the three participating offices, the supervisor - functioning as the primary facilitator of all SIA group meetings - made efforts to assure that each staff member was included in all SIA-related discussions and decision-making, and had a meaningful role to play in the office's SIA project. In several instances, a supervisor recognized that an individual or small group were dismissive of, or disruptive to, the office's project, and took steps to re-engage them in the group process.

During SIA, and in post-project interviews, the participating supervisors reported seeing benefits to giving their staff a strong voice. These benefits included some individuals gaining confidence and developing their talents; some coworkers developing new respect for, and closer working relationships with, one another; and the offices as a whole taking pride in sharing the important work they do with others in the community. 
Each of these positive developments served to increase the level of staff ownership in the office's SIA project. I learned that if I don't give a lot of initial input, and let people decide what they want to do, they come to really good conclusions and come up with good ideas. I think they own it more, too.

\section{Staff assuming responsibilities.}

During SIA, the majority of staff played an active role in their office's project. As is the case with many group efforts, some people made a commitment to complete specific tasks, and followed through; some people invested themselves more heavily in the project, and found themselves doing more than they originally promised to do; and a few people contributed little to their group's effort.

Some staff in each of the three participating offices reported that they and their coworkers were invested in their office's SIA project. It felt like more people took on the responsibility and tried to help out...rather than saying, "Oh, we have to do this." People had more ownership, and took more responsibility. For many staff, the successful completion of the SIA project became a priority. You know, you go for a job interview or something, there was that kind of energy, like we were really focused on getting this thing done. Some staff were pleasantly surprised by the level of commitment they saw in their office during SIA. In my previous office, one person was very clear: "I'm not a team player, I don't want to be." And when you have that happening, you're not going to have happen what we saw happen here. 


\section{Staff ownership increasing over time.}

In each of the three offices participating in SIA, staff's ownership of the SIA project grew as they moved toward their established goal. Even though there was a verbal commitment to do [an SIA project], it actually took a little while before I felt it really was owned by the group. Some individuals described how their commitment to the project increased as they began interacting with community partners. I sort of was only half-heartedly invested in it, initially. But as the Agency S liaison, I was actively involved in developing some of those relationships, and...it grew on me.

One supervisor reported that staff's ownership of their office's SIA project increased after they began working together in small groups. As they got more used to the idea, people began to own the process...And once they broke up into smaller committees, then I saw real commitment. Another supervisor explained that staff were somewhat resistant to the SIA project at first, but came to recognize the value of having a stronger voice in their work process. Initially they didn't embrace [the SIA project] as much as they did at the end. They realized that...by having a big say in what they do and how they do it, THEY can structure what they need to do anyway.

\section{Review.}

Staff's increasing ownership of SIA was a factor which facilitated the implementation of the learning organization model. Observations and comments indicate that, in each of the three participating offices, the supervisor believed in, and supported, the concept of staff ownership of office-level projects; staff took responsibility, 
individually and as a group, for completion of their office's chosen project; and staff's ownership of the SIA project increased as the project evolved.

\section{Research Question 3: What factors impeded the implementation of the learning organization model?}

\section{Storyline for Research Question 3}

The factors that were most influential in impeding the adoption of the modified learning organization model were: (a) agency structure; (b) office climate and culture; (c) lack of participant support for SIA; and (d) competing work obligations and time constraints.

Figure 5.5 - Storyline for Research Question 3

\section{Data Sources}

The three data sources analyzed to answer Research Question 3 were observations, interviews, and online survey open-ended questions. Table 5.12, below, summarizes which data sources contained evidence of which impeding factors.

\begin{tabular}{|l|c|c|c|c|}
\hline & Observations & Interviews & $\begin{array}{c}\text { Online Survey } \\
\text { Open-ended } \\
\text { Questions }\end{array}$ & $\begin{array}{c}\text { Online Survey } \\
\text { Closed-ended } \\
\text { Questions }\end{array}$ \\
\hline Agency Structure & $\mathrm{X}$ & $\mathrm{X}$ & $\mathrm{X}$ & \\
\hline Office Climate/Culture & $\mathrm{X}$ & $\mathrm{X}$ & $\mathrm{X}$ & \\
\hline Lack of Participant Support & $\mathrm{X}$ & $\mathrm{X}$ & $\mathrm{X}$ & \\
\hline $\begin{array}{l}\text { Competing Work } \\
\text { Obligations/Time Constraints }\end{array}$ & $\mathrm{X}$ & $\mathrm{X}$ & $\mathrm{X}$ & \\
\hline
\end{tabular}

Table 5.12 - Summary of data sources - Research Question 3

\section{Overview}

The remainder of this section of Chapter 5 is a detailed discussion of the factors which impeded the implementation of the modified learning organization model: agency structure; office climate and culture; lack of participant support; and competing work obligations/time constraints. A comprehensive outline of the discussion about Research Question 3 findings is provided in Figure 5.3 below. 


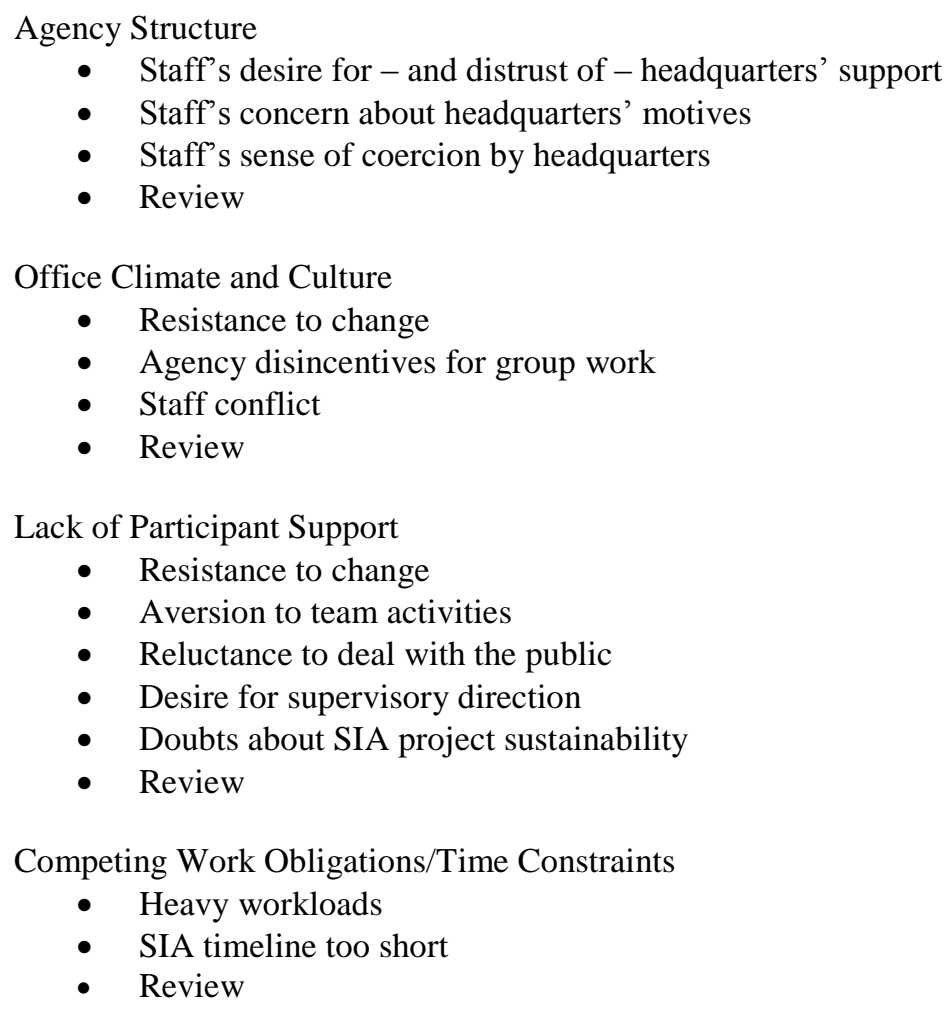

\section{Agency Structure}

VR's current organizational structure is similar to that of many publicly-funded social service agencies. The director of the agency and most managers of programs that service VR field offices state-wide are housed in a single office in a large urban area, and have limited day-to-day contact with staff outside headquarters. The majority of staff are located in field offices throughout the state, and are directly accountable to the supervisor of the office in which they work. The counselors in each office are responsible for serving the clients on their assigned caseload. The rehabilitation technicians in each office help the supervisor and counselors, in a variety of different ways, to accomplish their work. 
The hierarchical structure of VR, and the relationship that structure engenders between agency management and agency line staff, created some obstacles to the implementation of the modified learning organization model. The three areas related to agency structure that were most frequently noted as impeding factors were staff's desire for - and distrust of - headquarters' support for SIA; staff's concern about headquarters' motives for supporting SIA; and staff's sense that headquarters coerced them into participating in SIA.

\section{Staff's desire for - and distrust of - headquarters' support.}

At the beginning of SIA, before committing to working on an office-level project, some individuals in each of the participating offices asked for reassurance that headquarters fully supported the concept, and the implementation, of SIA. They were clearly hesitant to be part of any project that did not have the blessing of the VR director and headquarters staff. The supervisors and the staff are always thinking, "Is this what $V R$ wants to do?" They want to be sure that it's something that is supported, and that it's something that we should be doing.

Recognizing this as a key matter to address, the Program Manager and I spent a significant portion of the first meeting with each office's staff explaining the history of SIA, and the role the VR director and other headquarters staff played in authorizing and designing the project. Staff indicated they appreciated receiving this information. It helps to have somebody like [the Program Manager] come in and explain sort of what the reasoning is, and why it's important to do it. 
The Program Manager involved in SIA was a long-time headquarters employee known to, and respected by, the supervisors and many of the staff participating in SIA. The ongoing presence of the Program Manager in group meetings served to assure staff that, at the least, headquarters was not opposed to SIA. I knew if headquarters wasn't behind this, [Program Manager] wouldn't be here. Staff's belief in headquarters' support was also bolstered when, during SIA, the Program Manager carried their requests for project-related information, supplies or funding to headquarters, and their requests were granted.

However, the confidence built by the Program Manager's presence during SIA was undermined to some extent by the inattention SIA received from the agency director and other state-wide managers. Many SIA participants, including supervisors, noted that their office's SIA project did not appear to be a high priority for VR headquarters staff. Recently, we got an e-mail from [an agency manager] to complete the survey, but that was like [Information Technology], as I recall. Some staff made the point that headquarters' lack of interest in their office's efforts was not an exception, but the norm. We never see them or hear from them. They're just not even in our universe.

Staff commented on simple ways in which headquarters could have better supported their office during SIA. Some interest and attention...Some kudos from the state office to staff: "You're so busy, yet you volunteered to participate in [SIA], and this is just great that you're doing this"... that would have been nice. They felt their hard work on the SIA project was deserving of some public praise. We'd have liked to hear 
[headquarters] say, "Hey, look what the [deleted] office is doing," to have them put the office in the limelight a little bit, reward people for their efforts. That didn't happen.

All three of the supervisors involved in SIA underscored the importance of strong headquarters support for SIA and similar projects. Headquarters needs to cheerlead a little more if they want projects like [SIA] to really take off. In a post-project interview, one supervisor offered that if VR managers wanted SIA-type efforts to truly succeed, they would have to make them a clear priority from the top; name learning organization efforts as a legitimate way of doing business in field offices; hire someone at headquarters to oversee such efforts; and routinely provide facilitators to offer practical and moral support to supervisors and staff involved in learning organization projects.

\section{Staff's concern about headquarters' motives.}

As the SIA projects evolved in each office, staff began to openly discuss some of the beliefs they held when first introduced to SIA. Some staff indicated they thought SIA was just an attempt by headquarters to foist more work on an already overworked staff. In the beginning everybody was kind of like, "Who are they? What is this? Oh, just one extra thing to do."

Other staff admitted they initially thought headquarters selected their office to participate in the project because their office's performance was deficient in some way. The perception in the beginning was somehow our unit is bad, or we've failed in some way, and so that's why we have to do this project.

SIA participants' sense that headquarters intended to burden them with additional work or punish them for poor performance appeared to abate as the SIA projects evolved 
in each office. As staff began to concentrate their attention and energy on their office's chosen project, they tended to focus less and less on management's role in SIA. It became just us and the [office project]...we were focused on our [project] and our office being a success. Then you didn't hear so much about whether we were good or bad, or "Gee, we have to do this."

\section{Staff's sense of coercion by headquarters.}

At the beginning of SIA, the Program Manager, the supervisors, and I all made efforts to assure that the staff in each office decided whether or not to participate in SIA; what goal to pursue; and how to reach that goal. Care was taken to assure that all staff had a voice at every decision-making point in the process. Despite these efforts, both supervisors and staff later reported that some staff in each office felt headquarters had coerced their office into participating in SIA. I think they still felt that because we were being asked to do it that they were not necessarily forced, but they almost had to do it. And so I think initially they didn't embrace it as much as they did at the end.

In each participating office, staff made choices and took actions that established the direction, and impacted the outcome, of their office's SIA project. For the majority of staff, any initial feelings of coercion by headquarters were ameliorated by this level of direct involvement in the project. However, even having a strong voice in the process did not prevent some staff from continuing to feel manipulated by headquarters. Even though we were deciding what [the office's SIA project] was, we wouldn't have been doing a project if it was our decision, and so, the reason we were doing a project is because somebody else wanted one. 


\section{Review.}

Agency structure was a factor that impeded the implementation of the modified learning organization model. The agency's structure - a hierarchical model in which the agency director and state-wide managers work at a distance from, and have limited dayto-day interaction with, line staff - led to reactions which, at least initially, impeded progress toward the participating offices' chosen goals. These reactions included staff's desire for - and distrust of - headquarters' support of SIA; staff's concern about headquarters' motives for supporting SIA; and staff's sense of being coerced by headquarters into participating in SIA.

Office climate and culture was another factor that impeded the implementation of the modified learning organization model. The ways in which the existing climate and culture of the participating offices hampered the offices' SIA projects are examined below.

\section{Office Climate and Culture}

For an organization, or a workgroup within an organization, climate and culture are distinct but related concepts. Culture is a broad concept which includes the values, norms, and ways of behaving which organization members share (Deal \& Kennedy, 2000; Modaff, Dewine \& Butler, 2011; Ravasi \& Schultz, 2006; Rousseau, n. d.). According to Schein (1992):

Organizational culture is a pattern of basic assumptions - invented, discovered, or developed by a given group as it learns to cope with its problems of external adaption and internal integration - that has worked well enough to be considered 
valid and, therefore, to be taught to new members as the correct way to see, think, and feel in relation to those problems. (p. 494)

An organization's culture can incorporate such disparate elements its mission statement; its organizational structure (and who holds power within that structure); its reputation regarding trustworthiness; its effectiveness in delivering its product; and its office locations, furnishings, and art. Employees' dress, demeanor, interactions with one another, and interactions with outsiders are also aspects of an organization's culture. Harder for an outsider to see, but central to an organization's culture, are the "unspoken rules" that are well understood by, and guide the behavior of, long-term employees (Schein, 1992).

Although organizational culture is perceived and experienced by all who work within an organization or organizational subgroup, it is rarely referred to directly by the staff involved. In contrast, organizational climate is the work setting as described by the workers in the organization or workgroup. The worker's comments - solicited or unsolicited - often touch on such topics as communication, conflict, leadership, and presence or absence of workplace rewards (Rousseau, n. d.).

Certain aspects of the existing climate and culture in the offices participating in SIA impeded the implementation of the modified learning organization model. The three aspects of climate and culture that presented as the strongest impediments to implementation were staff's resistance to change; agency disincentives for group work; and staff conflict. 


\section{Resistance to change.}

Resistance to change is a pervasive phenomenon in organizations. Sticking with the "tried and true" often feels safer and more comfortable to staff than taking on unfamiliar tasks and new responsibilities. According to Senge (2006), "Resistance to change is neither capricious nor mysterious. It almost always arises from threats to traditional norms and ways of doing things" (p. 88).

During SIA, resistance to doing business differently was observed, and spoken of, as an office-wide reaction. Offices get a comfort level on how they're operating. Once they've established that, it's hard to find things to motivate and get people feeling like putting in the extra energy and effort to reach a new goal. It was also noted that certain individuals were particularly resistant to altering their regular work habits. There are $a$ couple of the old guard who just kind of went back to their cozy corners, and stopped thinking and stopped talking. But that's to be expected.

Resistance to SIA project activities was sometimes evidenced in non-verbal ways. In each of the three offices, at least one staff member spoke in group meetings only when spoken to, and responded primarily in monosyllables. Body language - folded arms, slouching postures, rolling eyes, or shaking heads - also sent signals that certain staff members were not engaged in or supportive of the office's SIA project. Fortunately, the initial resistance of some staff lessened as the SIA process evolved. In a few instances, individuals who appeared to be the least invested or most negative at the start of SIA became active and energized team members before the office's project was completed. 


\section{Agency disincentives for group work.}

For VR staff, sticking with the "tried and true" means doing more individual work than group work. Throughout SIA, staff in participating offices frequently noted that the approach being taken in their office's SIA project was not their "normal" way of doing business. We don't necessarily have that many projects that everybody has to be involved in. The counselors described the individual nature of their daily work. We really don't have a lot of responsibilities outside of our caseloads. This work tends to be pretty solitary - kind of you, your clients, your numbers. The work can be isolating sometimes.

Some staff suggested that both VR employees and the agency itself valued and rewarded individual efforts over group efforts. I do see us a bunch of rugged individualists, in a lot of ways, and I think VR promotes that. Several pointed out that VR counselors are evaluated exclusively on measures connected to their individual caseloads, not on their participation in group activities.

As each office's SIA project neared completion, there was discussion in group meetings about the gains staff had made during SIA, and the benefits of group work on a common project. Most staff agreed that the SIA project was worthwhile for both staff and clients, and indicated they would be interested in using a process similar to SIA for future office projects. However, many of those same participants stated that agency-wide changes would have to occur in order for their office to make effective use of such a model.

Specifically, some staff felt strongly that VR headquarters would have to recognize and promote group projects as a real and important part of their daily work. To 
get staff more involved, you'd have to think of a way to make it seem more integrated into their actual work obligations. This would include creating incentives for participating in group projects. Although staff suggested a wide a variety of possible incentives, ranging from compensatory time for conducting evening outreach activities to pizza parties for staff, by far the most frequently suggested incentives involved reducing counselors' heavy caseloads during group projects. If you want us to get into the group thing, you have to be prepared to take something else off our plate.

\section{Staff conflict.}

The conflict between certain staff members that existed prior to the start of SIA served to slow the progress of, and dampen the enthusiasm for, the offices' chosen projects. Supervisors and staff made it clear that bad feelings - from active animosity to more subtle signs of disrespect - existed between some individuals long before SIA began. I try not to get involved in whatever backbiting there is, because there's always some.

Especially in the early SIA group meetings, it was quite clear that ideas and information put forward by some staff were being discounted or viewed cynically by others. Rarely did one individual make a negative comment to another, but rolling eyes or side-bar comments while a person was sharing their perspective were not uncommon. Some staff indicated this type of behavior came as no surprise to them. They don't work well together, ever. Other staff offered specific descriptions of office personalities and dynamics. If "Susan" had taken the lead on [task] and decided she was going to make it 
work, that probably would have meant more happened. It also probably would have meant that a lot of people got angry, and it would have been very disruptive.

As the office projects progressed, some of the early tensions between certain staff members appeared to dissipate as they worked together on common tasks. Staff comments about improving relationships bore this observation out. However, the interviews and the online survey made it clear that SIA did not serve to mitigate all the conflict within the participating offices. Same divisive behavior along traditional turf lines.

\section{Review.}

The climate and culture of the offices participating in SIA was a factor that impeded the implementation of the modified learning organization model. Three specific aspects of climate and culture tended to slow the progress, and limit the effectiveness, of the offices' chosen projects: resistance to change (on the part of the offices as a whole, and certain staff in particular); VR's disincentives for group work; and staff conflict.

Lack of participant support for SIA was another factor that impeded the implementation of the modified learning organization model. The ways in which staff's lack of support impacted their office's SIA project are examined below.

\section{Lack of Participant Support}

The support of participating staff for their office's chosen SIA project was vital to the success of the project. The observed level of participant support for SIA varied widely. Certain staff members were enthusiastic about, and committed to, their office's project throughout SIA. Some staff were skeptical about the value of their office's SIA 
project at its inception, but developed interest in and commitment to the project as it evolved. Other staff were quite engaged in the early stages of their office's SIA project, but gradually stepped back after becoming discouraged by the actions, inactions, or poor attitudes of coworkers. Finally, there were staff who appeared to contribute as little as possible to their office's SIA project from beginning to end.

During SIA, and in interviews and survey comments, staff offered a variety of observations about lack of support for SIA. Some of their statements simply verified what had been clearly observed: Particularly at the beginning of the project, some staff regarded their office's SIA project as an imposition, and felt little commitment to it. They just saw it as something they were being assigned to do, and so they'd do their part, and participate, willingly, but they weren't invested in the results, and probably didn't feel the project itself was significant to them. Other comments pointed out that the lack of energy their office displayed for SIA was typical of their office's reaction to any project. Although everybody did what they were supposed to do, no one recognized it's going to have to be more than this if we're going to turn this into something...I think it would have been very hard to get that kind of energy level for any project.

Several participants suggested that the lassitude displayed by certain coworkers was simply a reflection of human nature. I think people were feeling, "I'll put in some effort but I won't put in my maximum effort." That's just the nature of some of the people. They're never going to put in their maximum effort on anything. Other staff offered more specific reasons why some participants were unsupportive of SIA, including: general resistance to change in the workplace; aversion to team activities; reluctance to deal with 
the public; desire for more direction from supervisors; and doubts about the sustainability of their office's SIA project.

\section{Resistance to change.}

As previously discussed under "Office Climate and Culture" above, some staff in each of the participating offices were resistant to playing an active role in their office's SIA project, either initially or throughout the entire project. This came as no surprise to some of their coworkers. You've got the old guard who want everything to stay the same. They don't want any changes, and that's always kind of a drag on things. Other staff believed that arrogance was the real reason some coworkers chose to contribute very little to the SIA effort. I think "Joel" was rather dismissive of the project because he felt he already knew it all.

\section{Aversion to team activities.}

As addressed in "Office Climate and Culture," above, the environments in which SIA participants work tend to reward individual efforts over group efforts. In the view of some staff, VR's long-standing focus on individual caseloads and individual outreach efforts has created a situation where VR staff are unskilled, and therefore uneasy, with teamwork in any form. I don't really think we really understand or value the effort of teamwork, in the true sense of what that really means. During SIA, some individuals made their aversion to working with a large group very apparent. She didn't want to team on stuff...she got the room, and she arranged all that, and then she went on vacation during the [event]! 
Another issue frequently mentioned was that staff feel uncomfortable and unsafe expressing their thoughts and feelings in a group setting. I think in the group everybody feels on the spot. They feel like they have to look like they're in agreement, even if they're not. Several staff suggested that, in any future group projects, individuals who feel reticent to speak in a group setting should be afforded alternative ways to convey their ideas. Maybe have people write down ideas on what the project should be instead of just talking about it at that meeting...so we can all have an opportunity to contribute.

\section{Reluctance to deal with the public.}

A large component of the SIA projects chosen by each of the three participating offices was outreach to individuals or organizations in the community. During SIA group meetings, some staff talked freely about their attempts - successful and unsuccessful - to connect with community partners. Other staff were much less forthcoming. For some, this was simply due to their shyness about speaking in group meetings. For others, though they had committed to contact certain community partners, it became evident they were making rather half-hearted efforts to follow through, or making no efforts at all.

Several interviewees discussed the difficulties that would inevitably surface when all staff were expected to do community outreach for the purposes of the SIA project. They noted that not all staff members are equally skilled in this area. There is a huge, huge discrepancy in people's ability to do [community outreach]. They also pointed out that, prior to SIA, staff who were uncomfortable making contacts outside the office were not pushed to do so. People who don't do [community outreach] well are just used to hanging back and letting other people do that sort of thing. 
When reminded that the Program Manager had suggested repeatedly at the outset of SIA that staff had the option of "teaming up" to complete any tasks involved in their project, one interviewee's immediate response was that some staff would perceive the need to team with others as a weakness. People who felt uncomfortable may have wanted to buddy up with somebody, but I don't think anybody would admit to needing that support. It's just hard. Another interviewee felt strongly that SIA facilitators should meet with staff individually before outreach activities begin. Find out where their comfort zone is. Because they're not willing to say that out in public. They're not going to say, "I'm really bad at public contact, and I don't want to do that." Don't assume everybody's comfortable.

\section{Desire for supervisory direction.}

As addressed in "Agency Structure," above, some staff were uncomfortable committing to participation in SIA until they were certain the project had the support and blessing of VR headquarters. During SIA, this tendency to look "up" for support and guidance was replayed at the office level.

Some staff made it clear they would have had more energy for, and commitment to, their office's SIA project if their supervisor had taken a stronger lead throughout their office's project. Have the supervisors more involved, active, and hands-on with the project. Several individuals expressed that their supervisor should have been more directive in the days and weeks between all-staff SIA meetings. They need to put in place those deadlines. Like "We're going to do this every week." Just don't wait for [Program Manager] and [researcher] to come to engage in the process. 
One interviewee spoke at length about how the supervisor had a responsibility to intervene when a few coworkers failed to commit to the office's SIA project, disrupted the process, and mocked the efforts of those who were committed to seeing the project through. It's a shame there was not enough clear leadership to address this but, again, that is who we work with here.

\section{Doubts about SIA project sustainability.}

Some participants were reluctant to devote energy to their office's SIA project because they did not believe any gains made during the project would be sustained once the project was completed. While some staff might try to retain some connections with the employers in the meeting, most of this will become a memory as more pressing things come up.

Some staff believed that if the Program Manager and researcher were not a continuing presence, none of the positives that came out of their office's project would continue. [We need] check backs over time. Because I feel like everybody's just dropped it now. We did what we were asked to, we did it, we turned it in, and it's over. Other staff believed that, whatever supports were available, their office would likely fail to do what was necessary to maintain relationships built during SIA. It's going to take effort, continued effort, on [VR's] part, to maintain that relationship...I mean, if it's $\underline{\text { not }}$ nurtured from our end, it's not going to continue. And I don't think it'll continue.

\section{Review.}

Lack of participant support was a factor which impeded the implementation of the modified learning organization model. Some participants' level of engagement in and 
commitment to their office's SIA project was consistently low. For others, lack of support was connected to one or more of the following: resistance to change; aversion to group activities; issues with community outreach; desire for more supervisory direction; or doubts about the sustainability of their office's SIA project.

Competing work obligations/time constraints was another factor which impeded the implementation of the modified learning organization model. The effect that heavy workloads and lack of time to devote to special projects had on SIA projects are examined below.

\section{Competing Work Obligations/Time Constraints}

The heavy workloads carried by VR staff impeded the implementation of the modified learning organization model. This was true from the point of early discussions about whether the offices would choose to participate in SIA (when concerns about finding sufficient time to complete an SIA project were voiced in each of the three offices) to the point that each office's project was complete. Time spent on SIA activities was perceived by staff as time taken from other work responsibilities, and this was observed to be the case.

Staff frequently made comments about their heavy workloads, including comments about their reluctance to add new responsibilities to their already burdensome work schedule. They also made many comments suggesting that the SIA project timeline was too short to accomplish what their office set out to do. 


\section{Heavy workloads.}

Whether or not they supported the concept of SIA, most participants acknowledged they were initially resistant to the idea of taking on any additional work obligations. I don't think anyone was particularly excited about even thinking of another project. They explained that such resistance was to be expected, given VR staff's increasingly heavy workloads. I think the average person feels overwhelmed with the work they already have, and to add one more thing on their plate...they see that as a problem.

Throughout SIA, in each of the participating offices, staff's concern about how to balance day-to-day work obligations and commitments to the office's SIA project was a constant refrain. Staff frequently made comments about having more work than they could handle, and finding it hard to find time to do tasks related to SIA. The push to write plans, close cases, increase our rehab rate, while not going over budget, was already there, while we were doing this project. Just the standard things, that kind of stuff, got in the way. They made it clear that their work pressures were waxing, not waning. I think people have been feeling very overwhelmed lately in terms of their workloads - many more clients coming in, and a lot more pressure for numbers and stuff from [headquarters].

Those staff who were most enthusiastic about, and committed to, their office's SIA project often expressed frustration that it was hard to find as much time as they would like to devote to SIA. I enjoy going out and making community contacts - I don't have a problem doing that. But I was already doing a lot... It was hard to find time to do 
[SIA activities] too. Several noted the paradox in their difficulty finding time to engage in their SIA-related responsibilities. We're caught in a Catch 22. We have all these people coming in that we have to deal with...we don't have time to go out and create new pipelines and work on marketing...that takes time, and energy.

By contrast, staff who felt some resentment about their office's involvement with SIA tended to cast their office's project as an impediment to their "real" work. In my opinion, this project just got in the way of the more important work we do.

\section{SIA timeline too short.}

In each of the participating offices, as the SIA project evolved, staff realized that the project they had chosen for themselves would take more time to accomplish than they anticipated it would. Contacting potential employers was a time investment that really wasn't considered before this project. Some commented about needing more time for very specific reasons. Didn't have contact with all the programs needed, due to problems scheduling all parties. Others spoke about time requirements in more general terms. We're not getting the right clients. So how do we get them? We get them by developing better relationships with referral sources. But the act of developing those relationships is very time consuming.

As the SIA projects progressed, participants often spoke of their growing awareness of the complexity of their office's project. [VR] has been identified as the place of last resort for people who have nowhere else to go. For us to turn that image around is going to take an enormous amount of work. Many staff suggested it would be 
beneficial if future SIA-type projects had a longer timeline. Have a more extended timeline, as it takes time to develop employer connections.

Some staff proposed that SIA-type projects should be purposely designed as ongoing efforts. In some cases, they felt strongly that the specific project their office had taken on for SIA should be - and deserved to be - continued. We shouldn't just drop it. Then it becomes an exercise...I don't think that's a good thing. I think we should continue on with the understanding that it's not a quick and easy kind of thing to do, to make those relationships work.

\section{Review.}

Competing work obligations/time constraints was a factor which impeded the implementation of the modified learning organization model. There were two specific areas that staff in each of the participating offices recognized, and frequently commented on: heavy "regular" workloads, which made it difficult for staff to devote time to their SIA responsibilities; and the SIA timeline, which many felt was too short for staff to fully develop the new strategies they agreed upon in group meetings.

\section{Research Question 4: What impact did the implementation (or partial implementation) of the modified learning organization model have on office climate and culture?}

\section{Storyline for Research Question 4}

The implementation of the modified learning organization model had the following impact on office climate and culture in the offices involved in the study: (a) supervisors assumed a more participatory role, granted their staff more autonomy, and found value in these changes; (b) staff relationships increased in both strength and number; and (c) offices improved their connections with community partners.

Figure 5.7- Storyline for Research Question 4 


\section{Data Sources}

The four data sources analyzed to answer Research Question 4 were observations, interviews, online survey open-ended questions, and online survey closed-ended questions. Table 5.13, below, summarizes which data sources contained evidence for which impacts on office climate and culture.

\begin{tabular}{|l|c|c|c|c|}
\hline & Observations & Interviews & $\begin{array}{c}\text { Online Survey } \\
\text { Open-ended } \\
\text { Questions }\end{array}$ & $\begin{array}{c}\text { Online Survey } \\
\text { Closed-ended } \\
\text { Questions }\end{array}$ \\
\hline Supervisors' Role & $\mathrm{X}$ & $\mathrm{X}$ & $\mathrm{X}$ & $\mathrm{X}$ \\
\hline Staff Relationships & $\mathrm{X}$ & $\mathrm{X}$ & $\mathrm{X}$ & $\mathrm{X}$ \\
\hline $\begin{array}{l}\text { Connections with Community } \\
\text { Partners }\end{array}$ & $\mathrm{X}$ & $\mathrm{X}$ & $\mathrm{X}$ & $\mathrm{X}$ \\
\hline
\end{tabular}

\section{Overview}

The remainder of this section of Chapter 5 is a detailed discussion of the impact the implementation (or partial implementation) of the modified learning organization model had on the climate and culture of the offices participating in SIA. The chapter ends with a note on the economy's impact on SIA. The term office climate and culture encompasses both observations of the manner in which staff approached challenges during SIA, and staff comments about their SIA experience.

The impacts that the implementation of the modified learning organization model had on office climate and culture are: impact on supervisors; impact among staff; and impact between staff and community partners. A comprehensive outline of the discussion about Research Question 4 findings is provided in Figure 5.4, below. 
Supervisors' Role
- Balancing supervisory responsibility and staff voice
- Ranaging staff resistance to SIA
- Review
Staff Relationships
- Staff and supervisor perceptions
- Factors facilitating staff relationships
- Responses to closed-ended survey questions
Connections with Community Partners
- Staff and supervisor perceptions
- Sustainability of connections with community partners
- Responses to closed-ended survey questions
Final Notes
- Variations in degree of improvement
- Effect of the economy

Figure 5.8 - Outline of Discussion of Research Question 4 Findings

\section{Supervisors' Role}

At the outset of SIA, the three supervisors agreed to have their staff learn about and consider taking part in - the SIA project. Once the staff in each office decided as a group to participate in SIA, the supervisor agreed facilitate their office's SIA process.

The SIA project was a clear departure from the offices' standard way of doing business. The supervisors' willingness to embark on an unfamiliar path was a strong indicator they were open to taking new and less hierarchical approaches to attaining office goals. The structure of the SIA projects required that participating supervisors encourage staff to express their project-related ideas freely, and that they give those ideas more weight than was normally the case. This was a new process for both the supervisors and staff, and it was not always smooth or comfortable in execution. 
Two specific supervisory challenges were frequently observed and commented upon. The supervisors' first challenge was striking a balance between their responsibility to guide the SIA project to completion, and their responsibility to grant their staff voice in the office's chosen project. Their second challenge was finding a way to effectively manage some staff member's resistance to the office's SIA project. A description of the ways in which supervisors addressed these challenges can be found below.

\section{Balancing supervisory responsibility and staff voice.}

Each of the three supervisors reported that, during SIA, they struggled to strike the proper balance between their responsibility to oversee their office's SIA project, and their commitment to allow their staff to have a strong voice in that project. They indicated that this was because their normal supervisory responsibilities and their SIA responsibilities - facilitator of the SIA process, and participant in their office's chosen project - differed.

Day-to-day, the supervisors were the clearly-defined leaders, and the voice of authority, in their office. In the context of SIA, their role was somewhat different. They were asked to facilitate their staff's journey through a new process, which definitely required their leadership skills. At the same time, they were asked to foster and support their staff's ownership of the office's SIA project, which required them to assume a more egalitarian role.

In their role as SIA facilitators, the supervisors felt a responsibility to guide the SIA process, so their staff's chosen project would not drift off course, and so the voices of some staff would not drown out or override the voices of other staff. They also felt a 
responsibility to be actively engaged in the office's chosen project - to function as a "team member" rather than a "team leader" - so staff could clearly see that they were invested in and supportive of both the project and the concept of creating a learning organization.

At the beginning of SIA, the supervisors found they had some personal difficulty relinquishing their normal leadership role. I wanted to get in there with the Employer Committee a little bit and start directing. They did not appear to misunderstand, or be resistant to, SIA's modified learning organization model. Rather, the supervisors, like their staff, had to practice and adjust to new ways of connecting and communicating in the office setting. Early on, I felt like I was trying to get too involved, trying to tell everybody what I thought we needed to do, and [later] I was able to take a step back.

Staff, used to relying on their supervisor's direction, contributed to their supervisor's initial difficulty in moving out of their established leadership role. They tended to refer to their supervisor for advice and information during SIA, as they did in their daily work. In the course of developing their office's project, staff often asked their supervisor's opinion. Is it really $O K$ for us to do that? They also frequently turned to their supervisor for practical information. If we want to move on this, who should we check with at Agency Z?

In many instances, the supervisor responded briefly and directly to staff inquiries, and then the SIA process continued. On occasion, the supervisor turned to the Program Manager for further information or guidance. In either case, it was observed in each participating office that certain individuals were more dependent on the direction of their 
"superiors" than others. Throughout SIA, it was evident that some staff were less inclined, or less able, than others to articulate their opinions and ideas without active encouragement from their supervisor or the Program Manager.

As the SIA project evolved in each office, and the participants became more familiar with the SIA meeting format and roles they had chosen to play in the office's project, the supervisors began to grant staff a stronger voice in group meetings and in the daily project activities. I let people get in there and decide what they wanted to do and what they needed to accomplish. Although the process was not always smooth, the supervisors saw real benefits in taking this approach. If you give them choice, informed choice, like "We're gonna do this, but you do have some choice," things are going to go better.

Throughout SIA, the supervisors wanted their staff to know they were committed to the office's goal, and willing to do their part to meet that goal. To this end, they worked side-by-side with their staff to complete certain project-related tasks. I was on the Program Committee, and I also wound up being on the Food Committee. At the same time, in their role as facilitator of the SIA process, they periodically found it necessary to step forward to deal directly with individuals or circumstances in order to keep the office's project moving forward. Although the dual role of "team member" and "facilitator" felt awkward at times, each of the supervisors reported that any discomfort they experienced was outweighed by the benefits - and anticipated future benefits - of SIA to their office. 


\section{Managing staff resistance to SIA.}

In initial discussions about the SIA project, the supervisors were provided an overview of current literature relating to learning organizations, which suggested initial staff resistance to the implementation of learning organization models was common. Each supervisor indicated they fully expected to encounter some resistance to participation in SIA, especially from certain individuals in their office.

During the course of the SIA project in each participating office, there was resistance from some staff, as has been described above. In addition to negative reactions during large group meetings noted earlier, some staff instigated disagreements or flatly refused to cooperate during small group activities, or failed to complete project-related tasks they had agreed to perform.

The motives for staff resistance were not always clear. Some motives that were articulated or observed were dislike of group process; having to cooperate with yet another administration-generated project; having any responsibilities added to an already large workload; and having to work cooperatively with disliked coworkers. In addition, some staff with plans to leave their position in the near future were frustrated they had to invest time and energy in a process that would not bear fruit before they departed.

The supervisors took various approaches to managing the resistance that emerged during SIA. They sometimes made efforts to present the staff's chosen project as a chance to move away from the "daily grind," to try new things, to have more autonomy than usual, and to have a good time. I tried to make it fun, not make it seem like some big burdensome thing. In many cases, they allowed the resistance to be voiced or displayed, 
acknowledged it, and then continued the meeting or activity. I also learned that even though people complain up front, not to pay that much attention to it, you know, just kind of move on from here. Like, 'This is what we're gonna do, so go ahead and complain a little bit, and then we're gonna move into action." In other cases, when the resistance was subtle - body language, or side comments - they simply ignored it.

In a few instances, the resistance of an individual to particular aspects of the staffselected project was so intense or disruptive that the supervisor found it necessary to address the resistance, either during staff meetings or in a private meeting with the individual. While it was not always possible to discern the individual's internal views about disputed issues, in each case he or she behaved less divisively in group meetings and activities from that point forward.

The supervisors reported that they were not surprised or disheartened by the resistance that staff displayed during SIA, as they knew their staff well and expected some "push back." They offered that some staff has actually been less resistant to, and more engaged in, SIA than they had anticipated at the outset of the project.

\section{Responses to closed-ended survey questions.}

The responses to five closed-ended online survey questions included in Table 5.14 , below, are pertinent to the discussion of approaches taken by supervisors during SIA. 


\begin{tabular}{|l|c|c|c|c|}
\hline \multicolumn{1}{|c|}{ Question } & $\begin{array}{c}\text { Number of } \\
\text { Respondents }\end{array}$ & Mean & $\begin{array}{c}\text { Standard } \\
\text { Deviation }\end{array}$ & $\begin{array}{c}\text { Agree or } \\
\text { Strongly Agree }\end{array}$ \\
\hline $\begin{array}{l}\text { The supervisor and staff in my office } \\
\text { worked together to select one short-term } \\
\text { goal for the project }\end{array}$ & 37 & 4.32 & .973 & $89 \%$ \\
\hline $\begin{array}{l}\text { The supervisor and staff in my office } \\
\text { each agreed to do one or more things to } \\
\text { help the office reach the goal }\end{array}$ & 37 & 4.22 & 1.109 & $84 \%$ \\
\hline $\begin{array}{l}\text { The supervisor and staff in my office } \\
\text { each did one or more things to help the } \\
\text { office reach the goal }\end{array}$ & 37 & 4.19 & 1.151 & $81 \%$ \\
\hline $\begin{array}{l}\text { My supervisor, coworkers and I worked } \\
\text { together on one or more parts of the } \\
\text { project }\end{array}$ & 37 & 3.83 & 1.056 & $80 \%$ \\
\hline $\begin{array}{l}\text { My supervisor, coworkers and I kept one } \\
\text { another informed of progress made on } \\
\text { the project }\end{array}$ & 37 & 3.95 & .941 & $78 \%$ \\
\hline
\end{tabular}

The strong positive responses to the five questions (ranging from $78 \%$ to $89 \%$ Agree or Strongly Agree) suggest that the supervisors were actively engaged in their office's SIA projects. These responses also suggest that the supervisors' interactions with staff during SIA were collaborative in nature.

\section{Review.}

The supervisors' dual role as the SIA facilitator and SIA team member was quite a departure from their day-to-day role as supervisor of their office, and required some practice and adjustment on their part. They found it particularly challenging to balance granting their staff strong voice throughout SIA and guiding the SIA project to a successful conclusion. They also found it challenging to manage staff's resistance as their office's project evolved.

Despite these challenges, each of the participating supervisors reported that the SIA project was, overall, a positive and worthwhile experience for their office. They saw 
clear benefits in taking a different approach to supervision, and said they would like to utilize a modified learning organization model in future group projects.

\section{Staff Relationships}

During the SIA project in each participating office, some staff worked together who had not had reason to connect - or connect in that way - before. In some instances, staff had worked for some time in the same office but in quite different roles, and had never collaborated on a work project before. Even staff sharing the same job title interacted sporadically, or only in passing. SIA offered staff an opportunity to work in concert with all of their coworkers, or with small groups of their coworkers. This served to increase and strengthen the bonds between coworkers within each office.

The staff relationships that developed as the SIA projects evolved were noticed and commented on by staff and supervisors. SIA participants' perceptions of the relationships that grew out of SIA, and the three factors that were key to facilitating those relationships, are discussed below.

\section{Staff and supervisor perceptions.}

Staff reported that they enjoyed the chance to work in concert with coworkers, and the camaraderie that developed, during SIA. Nice to work together on a common project. They also reported new awareness of coworkers' strengths. Made us look at individual strengths, and how we complement/help each other with strengths. They indicated that the SIA experience had forged new connections between coworkers that did not exist prior to the project. There were some bridges built. Because there tend to be little cliques, and some of the way the teams played out, I think there were interesting 
relationships built. In some cases, individuals spoke of plans to continue work on various projects, or to develop new projects, with particular coworkers they had "bonded with" during SIA.

The supervisors also reported that relationships had formed between certain staff members or among small staff groups during SIA. They expressed pride in their staff's ability to work together effectively on the office's project. They also indicated that they believed, and hoped, that the new connections made during the project would live on as a positive force for individuals and for the office as a whole.

\section{Factors facilitating staff relationships.}

Observations and participant comments revealed that several factors were key to the development of new relationships during SIA. The first two factors have been previously discussed: staff worked together rather than independently during SIA, and staff cooperated with one another while engaged in common activities. Everyone supported one another, and we got it done.

A third factor, less frequently mentioned but clearly observed, was staff's sharing of a common purpose. Teaming together to work towards a specific goal. For each of the three offices, the goal chosen and the approach taken to meeting the chosen goal were different. However, the uniqueness of staff purposely taking time and effort to work together was the same, and served to forge more and stronger connections among staff in each participating office. 


\section{Responses to closed-ended survey questions.}

The responses to one closed-ended online survey question included in Table 5.15

below, are pertinent to the discussion of new relationships among staff.

\begin{tabular}{|l|c|c|c|c|}
\hline \multicolumn{1}{|c|}{ Question } & $\begin{array}{c}\text { Number of } \\
\text { Respondents }\end{array}$ & Mean & $\begin{array}{c}\text { Standard } \\
\text { Deviation }\end{array}$ & $\begin{array}{c}\text { Agree or } \\
\text { Strongly Agree }\end{array}$ \\
\hline $\begin{array}{l}\text { The supervisor and staff in my office } \\
\text { worked together to select one short-term } \\
\text { goal for the project }\end{array}$ & 37 & 4.32 & .973 & $89 \%$ \\
\hline $\begin{array}{l}\text { My supervisor, coworkers and I worked } \\
\text { together on one or more parts of the } \\
\text { project }\end{array}$ & 37 & 3.83 & 1.056 & $80 \%$ \\
\hline $\begin{array}{l}\text { My supervisor, coworkers and I kept one } \\
\text { another informed of progress made on the } \\
\text { project }\end{array}$ & 37 & 3.95 & .941 & $78 \%$ \\
\hline $\begin{array}{l}\text { I learned something about the abilities } \\
\text { and/or interests of one or more of my } \\
\text { coworkers. }\end{array}$ & 37 & 3.59 & 1.066 & $60 \%$ \\
\hline
\end{tabular}

Table 5.15 - Closed-ended survey questions - New Relationships among Staff

The strong positive responses to the first three questions (ranging from $78 \%$ to 89\% Agree or Strongly Agree) suggest that staff in each participating office worked as a team during their offices SIA project. In addition, six out of ten respondents reported that their interaction with coworkers during the SIA project taught them something about the abilities or interests of coworkers. The increased interaction and awareness among coworkers creates the potential for stronger connections and collaborations in the future.

\section{Review.}

New relationships were created, and existing relationships were strengthened, between staff members and among small staff groups during SIA. Staff reported that they enjoyed the camaraderie that surfaced during SIA, they became aware of their coworkers' strengths, and they formed valuable connections with certain coworkers. Supervisors also noted staff relationships that emerged or grew stronger during their office's SIA project, and expressed pride in their staff's effective collaboration. Three factors that appeared to 
foster relationships were staff working as a group, staff support and collaboration during group activities, and staff sharing a common purpose.

\section{Connections with Community Partners}

As outlined earlier, each of the offices participating in SIA selected a goal that involved outreach to individuals or organizations in the community. The development of these VR/community partner relationships are discussed below.

\section{Staff and supervisor perceptions.}

Staff spoke of the groundwork their office had laid during SIA to create better connections in the community. We pin-pointed a specific way to help our office work smarter, and develop a better communication with other agencies. Some reflected on the overall value of the outreach efforts staff had made. This project opened doors to strengthen partnerships, which in turn better enabled us to help more customers. Others reported on the development of particular partnerships. We've created a more positive and open partnership with Agency S.

The supervisors reported that the SIA project helped them and their staff direct energy toward the important work of connecting with community partners. One supervisor spoke of her belief in the value of outreach work, her long-standing intent to pursue such work, and the way in which heavy workloads and emergent issues kept pushing outreach efforts to the side prior to SIA.

Each of the supervisors noted that their staff had learned, and were continuing to learn, a great deal from individuals and organizations they had connected with during SIA. They learned a lot that I don't think they would have otherwise. They noted that the 
information-sharing worked both ways, and community partners had requested and received a lot of valuable information from VR staff.

\section{Sustainability of connections with community partners.}

Some, but not all, of SIA participants anticipated that connections made with community partners during SIA could be sustained after their office's project was completed, and that these connections would lead to stronger relationships with community partners over time. In an interview conducted after her office's SIA project was completed, one supervisor reported with some enthusiasm that her staff continued to meet with staff in the organization they partnered with during SIA. They're meeting with Agency S regularly now, and they're going to see Agency S staff.

Some staff reported that the SIA experience had opened their eyes to the value of strong community partnerships. We were able to "see" the benefit of networking with a partner for ourselves and our customers. They expressed guarded optimism about sustaining the gains that had been made during SIA. It was helpful to see how developing strong employer connections can be beneficial for VR as an agency and for the community rehabilitation partners that we work with. We may be able to continue to develop more effective and efficient ways to do this.

\section{Responses to closed-ended survey questions.}

The responses to the two closed-ended online survey questions included in Table 5.16, below, are pertinent to the discussion of new connections between offices and community partners. 


\begin{tabular}{|l|c|c|c|c|}
\hline \multicolumn{1}{|c|}{ Question } & $\begin{array}{c}\text { Number of } \\
\text { Respondents }\end{array}$ & Mean & $\begin{array}{c}\text { Standard } \\
\text { Deviation }\end{array}$ & $\begin{array}{c}\text { Agree or } \\
\text { Strongly } \\
\text { Agree }\end{array}$ \\
\hline $\begin{array}{l}\text { I learned one or more ways individuals and } \\
\text { organizations outside VR (community } \\
\text { partners, employers, etc.) can help VR } \\
\text { customers }\end{array}$ & 37 & 3.84 & .764 & $68 \%$ \\
\hline $\begin{array}{l}\text { My supervisor, coworkers and I developed a } \\
\text { stronger working relationship with others } \\
\text { (VR customers, community partners, } \\
\text { employers, etc.) }\end{array}$ & 37 & 3.59 & 1.013 & $52 \%$ \\
\hline
\end{tabular}

Table 5.16 - Closed-ended survey questions - New Community Partner Connections

The positive responses to the first question (68\% Agree or Strongly Agree) suggest that most SIA participants felt they learned something from community partners that could help them better serve their clients (referred to within VR as "customers.") The responses to the second question (52\% Agree or Strongly Agree) indicate that more than half of SIA participants felt outreach activities during SIA resulted in stronger working relationships with others in the community.

\section{Review.}

During SIA, supervisors and staff in each of the three participating offices engaged in outreach activities designed to improve current relationships, re-establish former relationships, or build new relationships with community partners. They reported success in planning outreach efforts, contacting and learning from other individuals and organizations in their community, and forging stronger connections with old and new community partners.

Involvement in their office's SIA project convinced some participants of the importance of strong community partnerships. For others, participation in SIA served to underscore their established belief in the value of such partnerships. As SIA ended, they 
were hopeful they could sustain and grow the partnerships their office had started to develop.

\section{Final Notes}

\section{Variations in degree of improvement.}

While there were positive developments during SIA in each of the three areas addressed - supervisor's role, staff relationships, and connections with community partners - some areas showed more improvements than others. The responses to the closed-ended survey questions point to this difference: the responses to the questions referencing the supervisor's role were the most positive (78\% to 89\% Strongly Agree); the responses to the questions referencing staff relationships were also positive $(60 \%$ to 89\% Agree or Strongly Agree); and the responses to the questions referencing connections with community partners, while still positive, were less so (52\% to $68 \%$ Strongly Agree).

Several factors may account for the difference in the percentage of positive responses in the three areas. First, the supervisors and staff in each participating office started SIA with a common history - working in the same office, and sharing a strengths training experience just prior to the start of SIA - that they did not share with community partners. Second, while each of the offices chose to pursue SIA projects involving community outreach, supervisors and staff actually spent more time during SIA involved in planning meetings and group activities within the office than they did in the community. Third, the relatively short duration of the SIA projects did not allow the connections with community partners to fully evolve and mature. Finally, the primary 
focus of the research - including the online survey - was on the extent of implementation of the modified learning organization model and the interactions and experiences of the SIA participants. This focus may have created a bias in the responses to the closed-ended online survey questions.

\section{Effect of the economy.}

The SIA study was conducted in the midst of the Great Recession. I anticipated that poor economy would negatively affect the study participants and, by extension, the results of the study. In fact, participating staff's heavy workloads - increasing at the time of the study due to a hiring freeze and escalating client needs - did limit the time that staff could spend on SIA-related activities. However, the economic turndown also made some of VR's community partners more willing to collaborate on projects that would benefit common clients. This development is discussed in more detail in Chapter 6 (Discussion and Conclusions).

Research Question 5: In regard to the previous questions, what differences, if any, were found among the three VR offices participating in the study?

\section{Storyline for Research Question 5}

During SIA, some differences among the three participating offices were observed and/or reported by study participants during interviews or in response to open-ended online survey questions. The most notable differences were the approach taken by the supervisor; staff's sense of ownership in their office's SIA project; and the changes that occurred in each office's climate and culture during SIA. Analysis of the responses to the closed-ended online survey responses revealed no statistically significant differences among the participating offices.

Figure 5.9 - Storyline for Research Question 5

\section{Data Sources}

The four data sources analyzed to answer Research Question 5 were observations, interviews, online survey open-ended questions and online survey closed-ended 
questions. Table 5.17, below, indicates which data sources contained evidence of differences among the three offices participating in the study.

\begin{tabular}{|l|c|c|c|c|}
\hline & Observations & Interviews & $\begin{array}{c}\text { Online } \\
\text { Survey } \\
\text { Open-ended } \\
\text { Questions }\end{array}$ & $\begin{array}{c}\text { Online Survey } \\
\text { Closed-ended } \\
\text { Questions }\end{array}$ \\
\hline Supervisors' Approach to SIA & $\mathbf{X}$ & $\mathbf{X}$ & $\mathbf{X}$ & \\
\hline Staff's Sense of Ownership & $\mathbf{X}$ & $\mathbf{X}$ & $\mathbf{X}$ & \\
\hline $\begin{array}{l}\text { Changes in Office Climate and } \\
\text { Culture }\end{array}$ & $\mathbf{X}$ & $\mathbf{X}$ & $\mathbf{X}$ & \\
\hline
\end{tabular}

\section{Overview}

The remainder of this section of Chapter 5 is a detailed discussion of the differences found among the three participating offices: supervisor's approach to SIA; staff's sense of ownership; and changes in office climate and culture. A comprehensive outline of the discussion about Research Question 5 findings is provided in Figure 5.5, below.

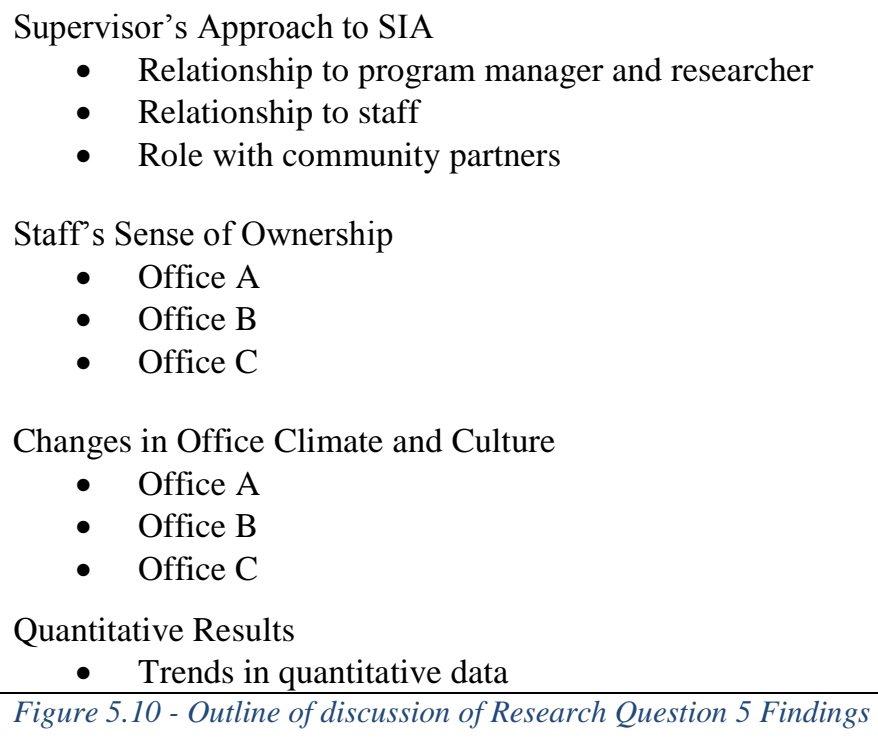




\section{Supervisor's Approach to SIA}

Each of the three supervisors participating in SIA took a somewhat different approach during their office's SIA project. A brief outline of the supervisors' interactions and styles is provided below.

\section{Relationship to program manager and researcher.}

Supervisor A's behavior toward the Program Manager and me was very welcoming and inclusive throughout SIA. Whenever we were in Office A, to attend an all-staff meeting or for another study-related purpose, she would greet us with a brief update on the experiences that she and her staff were having with their SIA project. Most of her stories were positive but, on those occasions when something was going wrong, she was very direct in describing the problematic situation. Between in-person visits, she would call or e-mail information she thought would be important for one or both of us to know.

When the Program Manager and I first explained the nature of the study and the steps involved in the SIA process to Supervisor A, she grasped the information we shared quickly and responded with insightful questions. She went on to assume the role of SIA facilitator with confidence and enthusiasm.

In the first two all-staff SIA meetings, Supervisor A referred to the Program Manager for clarification when staff posed questions about specific facets of the SIA process, or about headquarters' role in the process, that she was unable to answer. In later meetings, she appeared pleased whenever the Program Manager chose to offer her staff information or advice, but did not often solicit her input directly. 
Supervisor B had known and worked with the Program Manager on several previous organizational projects, and clearly respected her expertise. (Supervisors A and C had met the Program Manager at agency meetings and trainings, but had not worked with her directly before SIA.) She indicated when the Program Manager and I first met with her that she had reservations about SIA and her staff's ability to benefit from it. At the same time, she was clearly pleased the Program Manager and her colleagues at headquarters had considered including Office B in the study. When interviewed at the conclusion of SIA, Supervisor B offered that she would have decided against Office B's participation in SIA if the Program Manager had not been involved in the project.

Throughout SIA, Supervisor B utilized the Program Manager's support in a variety of ways. She referred to her for information and advice about SIA matters during all-staff meetings, as well as in private conversations and e-mail inquiries. She asked her to access specific supplies and resources at headquarters for use in Office B's SIA project. In addition, she had the Program Manager step in and facilitate certain portions of SIA all-staff meetings on several occasions, a pattern not seen in the other two participating offices. Without overstepping any established boundaries, Supervisor B also found ways to acknowledge my presence and utilize my support during SIA.

Both in SIA meetings and in the privacy of her own office, Supervisor B often thanked the Program Manager and me for the support we provided in Office B's SIA project. When interviewed at the conclusion of SIA, she said there should be many more projects like SIA in which headquarters provided similar on-site supports to supervisors and their staff. 
Supervisor C was pleasant but cautious with the Program Manager and me during our initial meeting to propose Office C's participation in the study. Although she listened attentively, she made it clear she would not consider participating in SIA unless her staff unanimously approved. Some time elapsed before she reconnected me with the decision she and her staff had made.

During the first two SIA all-staff meetings, Supervisor C asked the Program Manager and me many questions about the SIA process, and what headquarters would be expecting and requiring of her and her staff. However, once she and her staff selected their goal of collaborating with Agency S and began to sort out the particulars of their project, she visibly relaxed. Her attention turned away from the Program Manager and me, and she began to actively guide her staff through discussions about the merits of various approaches to their target agency.

When interviewed at the completion of SIA, Supervisor C said that upon reflection it was very important to her that the Program Manager and I had been present at the early SIA meetings to provide specific information about the study that she and her staff needed to feel confident moving forward with their project. However, she said our presence was less critical - but no less welcome - as time went on, because she and her staff felt comfortable with and capable of accomplishing the goal they had established.

\section{Relationship to staff.}

Supervisor A took clear charge of each large group SIA meeting, making sure each staff member had a voice in each stage of the office's SIA project. In meetings, she 
actively engaged with her more vocal staff, "drew out" quieter staff, and deftly addressed the negativity of certain individuals in order to move the process forward.

As previously described, Office A chose to create a number of small committees to handle different facets of the office's SIA project. Supervisor A functioned as a working member of the smaller committees, and kept close track of the activities of each of the smaller committees throughout SIA. Both during and after SIA, staff commended her for her enthusiasm, support, and willingness to put herself "on the line" to make the office's project a success.

Especially at the beginning of SIA, it appeared that some of the staff were doing their part in the office's project less because of personal interest and more to please their supervisor. As time went on, most staff became increasingly engaged in their chosen project activities, but obviously extended themselves to complete certain actions out of respect for their supervisor.

Supervisor B, a forceful personality, had been in charge of Office B for a short amount of time when SIA started. She informed the Project Manager and me that she was still working to understand the history and culture of the office, and the impact of her arrival and supervisory style on certain individuals and the office as a whole.

The dynamics of Office B shifted once again when, shortly before SIA started, a nearby field office was closed and several staff from that office were transferred to Office B. In contrast to Offices $\mathrm{A}$ and $\mathrm{C}$, where relationships - for better or worse - were already quite well-formed, supervisor and staff in Office B were still finding their way with one another during SIA. 
In the first few group meetings, Supervisor B's attention appeared to be more focused on the Program Manager than on her own staff. Her efficient and intensely practical approach to SIA did not give rise to extended group discussions; in fact, she sometimes seemed impatient with staff who expressed their SIA-related ideas or opinions at any length. As Office B's SIA project moved forward, Supervisor B sometimes invited the Program Manager to facilitate portions of the office's SIA group meetings. When she was not acting as facilitator, her comfort level and level of engagement with staff both appeared to increase.

Although Office B staff spent less time outside of SIA group meetings working together than staff in Offices A and C, several staff members got quite involved with and energized by the connections they made with community partners during SIA. They began sharing their experiences in the community with other staff members or with their supervisor between group meetings. These discussions led to a number of ideas for internal processes or external collaborations staff wished to pursue beyond SIA. The Program Manager and I were struck by the pleasure Supervisor B expressed about her staff's plans and her willingness to mentor these efforts beyond SIA.

Supervisor $\mathrm{C}$ takes her position and her responsibility to staff very seriously. It was clear from the outset of SIA that she and the majority of her staff had a long history and a warm and strong connection. Supervisor and staff communicated freely and easily, and their discussions about various aspects of their chosen SIA project were more animated and more layered than those in the other participating offices. 
In Office $\mathrm{C}$ more than in others, the reticence that existed in early SIA meetings proved to be staff adjusting to the unfamiliar - the Program Manager, me, and the SIA process - rather than staff learning about each other or about working together. This was not surprising, as the subgroups involved in pushing the SIA process forward at each of the three sites worked together in small offices and in small communities on a daily basis. In some cases, they had been coworkers in the same environment for many years.

In her role as facilitator during SIA, Supervisor C gradually shifted from being the clear director of the process to granting her staff more and more voice in, and increasing responsibility for, various aspects of their SIA project. However, understanding she had the most entrée with the supervisors and administrators of Agency S (the organization that was the focus of Office C's SIA project), she consistently and actively opened doors so her staff could access their colleagues in the other agency.

At the close of SIA, Supervisor C expressed pleasure and pride in what her staff had accomplished. She also reported that giving her staff more voice during SIA had turned out to have many benefits for her, for her staff, and for the office's clients. The benefits she outlined included all staff (including her) having an increased awareness of and confidence in one another's capabilities, and staff from Office C's three sites having a stronger sense of being one effective working team.

\section{Role with community partners.}

Office A chose building connections with local employers as their SIA project. Supervisor A let staff on the employer outreach committee make all of the initial contacts with employers, during which they invited the employers to an Office A-sponsored 
business breakfast. However, as a member of the programming committee, she contributed a lot of ideas and energy to the planning of programming to be presented at the business breakfast; as a member of the food committee she negotiated with headquarters for a food budget and shopped for food and beverages to serve at the breakfast; and, in her role as office supervisor, she hosted the business breakfast presentation.

Office B decided to contact or re-contact other human service organizations in the community to educate them about the type of client referrals VR is able to accept, and to solicit such referrals. Staff decided as a group that each staff member, including Supervisor B, would select and take responsibility for contacting at least one organization. Supervisor B and one other staff member chose to work together in contacting one of the larger human service organizations in the community, and met with administrators and staff from the other organization several times during SIA. Her role in the community was the same as the others on staff once the project began, although she may have had an easier time setting up initial meetings with her chosen organization given her status and level of recognition as supervisor.

Office $\mathrm{C}$ elected to work on collaborating with another human resources agency Agency S - during SIA, in an effort to improve services for the two organizations' common clients. Supervisor C made initial contacts with Agency S administrators and the Agency S supervisors in each of the three communities where Office C staff are sited. She presented the nature and intent of Office C's chosen SIA project, and scheduled initial meetings between her staff and Agency S staff in each of the three communities 
that Office C serves. Thereafter, Supervisor C encouraged staff at each Office C site assume a strong role in building connections with their Agency S colleagues, although she continued to attend all formal meetings scheduled with Agency S, remained interested in and informed about her staff's interactions with Agency S, and supported her staff in any way she could.

\section{Staff's sense of ownership.}

Staff's sense of ownership in their SIA project, and the path they took to achieve that sense of ownership, varied among the three participating offices. The experience of each office in regard to staff's sense of ownership is examined below.

\section{Office A.}

Office A was the first office that agreed to participate in SIA. Despite staff's early, unanimous decision to be part of the study, many staff did not appear to be fully engaged during the earliest SIA meetings. It was later reported that some staff in Office A initially agreed to be part of SIA because they thought it was what their supervisor wanted, or what headquarters expected their supervisor to accomplish. Simply put, they were cooperating out of respect for her.

According to Supervisor A and several of her staff, the turning point in Office A's SIA project was when staff decided to break up into small committees. Each committee took responsibility for one aspect of the business breakfast they were putting on for local employers. When staff member recognized that they and a handful of their coworkers had accepted responsibility for accomplishing a particular SIA-related task within a very specific timeframe, it focused their attention and forced them to begin interacting with 
other committee members. In some instances, the individuals on a committee had never had reason to work together before.

As Supervisor A and staff members reported, and as observed during SIA group meetings, once the committees started working on their chosen tasks, some staff's original view that they were being coerced into participating in SIA by a hidden agenda coming from their organization's headquarters started to dissipate. Instead, staff began to speak of the business breakfast they were planning as their project, and spent their time in both small group and large group meetings sharing progress they had made and problems they had encountered as they attempted to accomplish their committee's goals.

By the time the business breakfast took place, the whole Office A staff were clearly proud of the fact the office had come together and done a commendable job, both in setting a beautiful scene and in effectively presenting the important work their office does to local employers. In interviews and in online survey comments, a number of staff made positive comments about stronger working relationships they had formed with certain coworkers during SIA, and a newfound sense of camaraderie in the office as a whole.

\section{Office B.}

In Office B, when the Program Manager and I first met with the entire staff and explained the SIA study, only a few staff asked questions, responded to our questions, or spontaneously offered information or opinions about how their office conducted business or how their work processes could be improved. At the end of the meeting, Supervisor B 
expressed her frustration about the handful of staff who actually engaged in the meeting, suggesting they were - as usual - effectively drowning out the voices of their coworkers.

Over the course of the first two all-staff SIA meetings, the more vocal staff appeared to develop an awareness of their supervisor's frustration with them, and stopped speaking as freely within the group. Unfortunately, this did not have the effect of encouraging newer and less confident staff to speak up. In fact, staff interactions in Office B's remaining SIA meetings were more cursory and less complex than those in either Office A or C.

Although communications during SIA group meetings in Office B were less than ideal, some staff experienced and reported much more energizing and fulfilling interactions with staff at the human services organizations they contacted in the larger community. Their outreach to other organizations resulted in new client referrals for the office, as well as some new opportunities for staff to collaborate creatively with staff in other agencies or businesses.

For some staff, participating in SIA-related outreach activities led to thoughts about more efficient ways their office could handle in-house work processes, or more effective ways to do business with community partners in the future. While they did not tend to share their ideas in group meetings, they did begin to seek out and share ideas with their supervisor and selected coworkers. Before SIA concluded, several small staff groups were planning to try new approaches at work in the near future, and they had the full support of their supervisor in doing so. There was a strong sense that some staff had found a way to make a small piece of SIA their own. 


\section{Office C.}

Office $\mathrm{C}$ took longer than the other participating offices to agree to participate in SIA. By the time the Program Manager and I were invited into the office, Supervisor C had given her staff an overview of the modified learning organization model, and staff had already been talking together about what the office's SIA project might be.

Supervisor and staff were polite and attentive as the Program Manager and I provided them with additional information about SIA. They asked many questions about the modified learning organization model, the process they would be using during SIA, and headquarters' expectations for their office during the study.

From that point forward, there was a clear and observable upward trend in staff's sense of ownership of their SIA project. Once supervisor and staff felt they understood the intent of SIA, their focus shifted away from the Program Manager and back to their working group. In early meetings, Supervisor $\mathrm{C}$ facilitated discussions about SIA in a calm and confident fashion, making sure that each staff member had a "say" in each decision made. In later meetings, the supervisor sat back - both literally and figuratively - and allowed staff members from each of the office's three sites lead the discussion as they reported on SIA developments, shared information and insights, and made decisions about adjusting their project's course.

At the conclusion of SIA, both the supervisor and staff indicated staff interactions during the SIA process and the connections made with Agency S were both much more successful than they had anticipated. They said the whole idea of SIA seemed awkward at first but, as time went on, the SIA project really began to feel like their own. When asked 
if they would consider using the modified learning organization model for future office projects, they answered with an unqualified "Yes."

\section{Changes in Office Climate and Culture}

Although there were some changes in office climate and culture observed and reported in each participating office, the changes differed in each location. The nature of the changes in each office are described below.

\section{Office A.}

In Office A, the most significant change was in the quality of connections between coworkers. Prior to SIA, friendly and contentious relationships both existed in the office, but staff tended to focus day-to-day on their individual work responsibilities. The SIA experience overall - and the time spent working on SIA committees in particular - provided staff a chance to learn more about their coworkers as people, become aware of their skills and talents, and learn how to work effectively with them. Camaraderie grew as the office's SIA project progressed, and reached a peak during the office's business breakfast. Altogether, participation in SIA served to strengthen bonds and warm relationships among Office A staff.

\section{Office B.}

In Office B, there was little evidence of change in climate and culture office-wide. However, during SIA, staff made positive connections with staff in other organizations that laid the groundwork for long-term collaborations with those organizations. These outreach efforts gave several staff ideas for future changes in both internal and external 
Office B practices. When they shared their ideas with their supervisor and selected coworkers, the ideas were well-received and strongly supported.

Altogether, Office B's SIA project served as the impetus for some staff to develop stronger working relationships with community partners. In addition, it led them - with the support their supervisor and selected coworkers - to invest themselves in developing new practices likely to energize their work life and benefit their workplace.

\section{Office C.}

In Office $\mathrm{C}$, the biggest change and clearest benefit SIA brought to the office was staff's new method of communicating in staff meetings. Office C's staff work on a daily basis in three different work sites, and come together once each month for an all-staff meeting. Prior to SIA, this meeting was primarily used by the supervisor as a vehicle to dispense information from headquarters and discuss mandated workplace changes.

During SIA, in all-staff SIA meetings, Office $\mathrm{C}$ developed a specific pattern of communication. Staff from one site would report how matters relating to the SIA project were progressing at their site, and then throw the conversation open to all for suggestions and discussion about what might be the best "next steps" for the site. The process would then be repeated for the second and third sites.

At the conclusion of SIA, Supervisor C and her staff reported that Supervisor C still shares information of importance to staff at monthly meetings. However, in addition, staff routinely talks together about developments at each of the three sites, in much the same way as they had communicated during SIA. They see this new pattern of communication as a very positive change for the office. 


\section{Quantitative Results}

A one-way between-groups analysis of variance (ANOVA) test was conducted for each of the 14 closed-ended online survey questions (Appendix I). Only the question, "My supervisor, coworkers and I kept one another informed on progress made on the project" approached statistical significance at the $\mathrm{p}<.05$ level, at .071 . However, Levene's Test of Equality of Error Variances showed a lack of homogeneity for that variable, at .028; a Welch test conducted on that variable showed a significance of .143 (not significant); and the non-parametric Kruskal-Wallis test showed a significance of .128 (not significant). The Kruskal-Wallis test was also conducted for the other 13 closedended questions (Appendix J), with no significant findings. In sum, analysis of the responses to the 14 closed-ended online survey questions revealed no statistically significant differences among the three offices participating in the study.

\section{Trends in quantitative data.}

As noted above, the differences in responses to the closed-ended online survey questions among the three participating offices were not statistically significant. Because the number of participants in each office was small, I examined the descriptive data (Appendix K) to see if there were perceptible patterns in the quantitative data.

Looking at the responses across the three offices, I noted that the responses to the four fidelity statements were more positive - averaging over 80\% Strongly Agree or Agree - than the responses to either the learning or communication and collaboration statements. This supports the conclusion that the modified learning organization model was adopted by all three offices, despite the offices' distinct cultures, the different 
projects chosen by each office, and the unique approach each office took to completing its project.

Overall, the responses to the five learning statements - focused on respondents' acquisition of skills, self-knowledge, information about co-workers, and increased awareness of ways to assist clients - were most positive in Office C. This fit with my observations that the staff in Office $\mathrm{C}$ acknowledged and discussed personal and team learning more often during SIA than staff in the other two participating offices. At the same time, Office B had strong positive responses to the questions about taking on new tasks (78\% positive) and learning about how community partners can assist clients (78\%). These responses clearly reflected their chosen SIA project, which involved Office B staff contacting community partners who - in most cases - they had not interacted with before. Office A had the least positive responses to the learning statements. My observations during SIA, and information Office B staff provided in interviews, suggest that this may be partly due to a particularly strong sense of competence this group of workers brought to their office's SIA project - a sense not shared to the same degree by staff in the other two participating offices.

Regarding the five communication and collaboration statements, Office $\mathrm{C}$ had the most positive responses overall. This makes sense, as the office climate and culture prior to SIA was such that the staff had more experience working effectively as a team than staff in either Office A or Office B. Not surprisingly, Office A, the office that chose to break into small groups to work on the SIA project, gave the most positive responses to the statements about working together and keeping one another informed of progress. 
Office B had fewer positive responses to the communication and collaboration statements in general. This likely reflected the fact that, during their office's SIA project, each staff member went into the community on their own to contact individuals or organizations. It may also have reflected the fact that Office B had just integrated several staff members from another office, and the workers in the office had less history with, and trust in, one another than workers in Office A and C.

\section{Summary of Findings - Research Questions 1-5}

\section{Was the modified learning organization model implemented?}

Strengths in Action (SIA), a modified learning organization model, was implemented in each of the three VR offices that participated in the study. In each office, the majority of staff engaged in all five learning organization disciplines - personal mastery, mental models, building shared visions, team learning, and systems thinking.

Personal mastery was in evidence when staff took on tasks and roles they had not assumed before, and increased their confidence and expanded their expertise by doing so. It was also visible when staff learned about coworkers' and community partners' strengths and working styles, and used this knowledge to develop more productive ways to collaborate with them. Mental models could be discerned when staff altered the way they viewed and approached their own work, changed the way they interacted with coworkers or community partners, or developed a new perspective on particular work processes. Building shared visions was apparent when all staff members in each office participated in the discussion and decision-making that led to the selection of their office's SIA goal. 
Team learning was the discipline most frequently observed and commented upon during SIA. It could be seen when staff learned things - sometimes from one another, and sometimes from community partners - that were of use and value to workgroup. The acquisition and sharing of knowledge and skills served to foster pride, boost morale, increase motivation, and promote a sense of support and connectedness in individuals and in the workgroup as a whole. Systems thinking was evident in staff's sharing of information and ideas throughout SIA; staff's recognition and use of their coworker's personal strengths; and staff's comments on the value of SIA and its modified learning organization model to them and their workgroup.

\section{What factors facilitated implementation?}

Five factors were particularly powerful in facilitating the adoption of the modified learning organization model. One factor was the structure of SIA. The elements of SIA's structure that participating offices found most beneficial were the time and space SIA afforded them to pursue a group project; the involvement and contributions of the Program Manager and researcher; the fact that SIA participants were held accountable; and the focus on using staff 's personal strengths during SIA.

A second factor was supervisor support. Supervisor support during SIA took many forms, including "hosting" the Program Manager and researcher, facilitating SIA large group meetings, participating in small SIA workgroups, and overseeing SIA between formal meetings. In addition, supervisors assured all staff had a "voice" in the process, encouraging staff voice, and mentoring participation.

Growing connections among staff was a third factor that encouraged implementation. As staff worked together on their office's SIA project, there was an 
increase in communication, camaraderie, and mutual support among coworkers. These positive developments then served to increase staff's interest and level of engagement in their common project.

A fourth factor, similar in nature to the third, was growing connections between staff and community partners. Each of the SIA projects selected by the participating offices involved some form of outreach into the community. As staff contacted individuals and organizations outside their workplace, the relationships that were forged, and the potential for mutually beneficial collaborations, increased staff's enthusiasm for and engagement in SIA.

Staff's increasing ownership of the SIA project was the fifth factor that facilitated the implementation of the modified learning organization model. As the SIA project in each participating office moved forward, there was an increasing sense on the part of staff that it was "their" project. Supervisors' support and encouragement of staff contributed to staff's ownership of the project. Staff's ownership was also due in part to staff's assumption of project-related roles and responsibilities. These activities afforded staff affirmation and success, and made them feel a real and important part of the process.

\section{What factors impeded implementation?}

Four factors stood out as impediments to the adoption of the modified learning organization model. One factor was agency structure. At the outset of SIA in each office, some staff required assurance that the agency director and area administrators approved of their participation in the study. Some staff suspected headquarters' motives for supporting SIA, and others felt pressure to participate because of headquarters' support. 
A second factor was existing office climate and culture. In each office, some staff had a reputation for resistance to workplace changes. Others had a strong preference for working alone, which is not surprising given that most workers are responsible for individual caseloads and the agency measures individual - not group - performance. Whether active or passive in nature, long-standing conflicts between certain staff members also worked against a smooth SIA process.

Lack of participant support - closely related to climate and culture - was a third factor that served as an impediment. During SIA, some staff resisted change to their established work patterns, were actively or passively averse to participating in group work, were uncomfortable with the lack of supervisory direction, or limited their engagement in SIA because they doubted its sustainability.

A fourth factor that stood in the way of implementation, clear and constant in each of the three offices, was competing work obligations and time constraints. While SIA was operating, supervisors and staff remained responsible for managing their usual heavy workloads, with all the attendant pressures and distractions. This created a situation where even the most enthusiastic SIA participants had limited time between group meetings to devote to SIA-related activities. Because of this, some staff suggested some work obligations should have been lifted to accommodate SIA activities, or the SIA timeframe should have been longer.

\section{How did SIA impact office climate and culture?}

SIA impacted the climate and culture in the participating offices in three primary ways. One impact was on the supervisor's role. During SIA, the office supervisors were 
asked to assume responsibility for facilitating SIA group meetings and leading an extended team process. Each supervisor, used to being the office's undisputed leader, reported some difficulty "guiding" SIA effectively and managing staff resistance while, at the same time, giving staff sufficient voice in the process. In the end, each supervisor felt the SIA project had clear benefits for the office - including improved connections among staff and stronger collaborations with community partners - and indicated she would like to use the modified learning organization model in future group projects.

A second impact was on staff relationships. Relationships were formed, and existing relationships were strengthened, as staff worked together during SIA. Staff committing to participate in a team-based effort, cooperating and actively supporting one another, and sharing a common goal all served to deepen understandings and increase interactions within each office.

Stronger connections with community partners was a third impact SIA had on office climate and culture. In different ways, community outreach was central to each office's SIA project. The supervisor and staff in all three offices reported that during SIA they had interacted and shared information with staff in a variety of organizations in their community. They expressed confidence that their efforts had created or strengthened partnerships with these organizations, and laid the groundwork for future collaborations.

\section{Were there differences among the participating offices?}

During SIA, there were three clearly observable differences among the three offices. All three differences were in areas often mentioned during SIA, and frequently commented upon in interviews and in the online survey when SIA concluded. 
One difference was the supervisor's approach to SIA. Each supervisor had a unique working relationship with the Program Manager and the researcher. The relationship between supervisor and staff played out differently in each of the three offices. In addition, each supervisor played a distinct role with community partners during the office's SIA project.

A second difference was staff's sense of ownership in their office's SIA project. In each of the three participating offices, staff's sense of ownership in their office's project increased rather than decreased during the course of SIA. However, there were some discernible differences in the path staff took in each office.

In Office A, staff began to develop a real sense of ownership in their SIA project after breaking into small committees to work on different aspects of the office's planned business breakfast. The office was unique in having a culminating SIA event - the business breakfast - shared by all staff. This fostered pride and served to create a group sense of ownership not seen to the same extent in Offices B and C.

In Office B, the sense of ownership that developed for certain staff members was connected less to interactions with coworkers, and more to interactions with community partners. However, by the end of SIA, some staff were bringing ideas that grew out of their experiences in the community back to their supervisor and coworkers, and they were working together planning future changes to workplace practices.

In Office C, Supervisor C started by facilitating her office's SIA project in a calm and confident manner. As her staff started work on their chosen project, and she granted 
them increasing voice and autonomy, they developed a stronger and stronger sense that the project was their own.

Change in office climate and culture was the third difference between the participating offices. In Office A, the most apparent change was in the connections between coworkers. In an office where staff had a reputation for being "lone rangers," SIA served to create stronger bonds and warmer relationships among staff.

In Office $\mathrm{B}$, the most obvious change from business as usual was staff being encouraged to reach out and make positive connections with community partners. For some staff, these outreach activities generated ideas for changes in Office B's practices. Before SIA was completed, those staff had consulted with their supervisor and coworkers, gained support for their ideas, and begun planning to implement new processes in the workplace. In sum, SIA had a positive impact on some staff's relationships, both in the office and in the community, and added a new dimension to their work life.

In Office $\mathrm{C}$, the most obvious and most beneficial change during SIA was staff's introduction to a new method of communicating in staff meetings. Prior to SIA, the office's monthly staff meetings were primarily a way for Supervisor C to share information from headquarters with her staff. During SIA, staff from all three sites got comfortable with and adept at sharing SIA-related information. They soon began to take the same approach when other issues arose in monthly staff meetings, and commenting on the value of sharing perspectives and using one another's expertise. 


\section{Chapter 6: Discussion and Conclusions}

The chapter contains six sections. It begins with an examination of the key

findings of the research. This is followed by discussions of implications, my role as researcher, research limitations, and directions for future research. The final section is a summary of conclusions drawn from the research.

\section{Key Findings}

SIA succeeded on a number of different levels. The modified learning organization model was implemented in the three participating VR offices. The offices' SIA projects were a new experience for VR staff, both because they most often worked independently and the SIA project was a team effort, and because they had a stronger voice in the project than they normally had in office affairs. Each staff member was proactive in choosing to participate in the SIA study, helping to select their office's goal, and working with coworkers and community partners to achieve that goal. As their chosen SIA projects evolved, both staff and supervisors took on roles and tasks outside their normal work responsibilities, which served to increase their knowledge, skills, and confidence.

During SIA, staff in each participating office worked closely together and functioned as colleagues, not competitors. In doing so, they learned things about their coworkers' strengths and interests they had not known before. This led to better staff communication, more productive teamwork, stronger relationships, an increase in camaraderie, and improved office morale. 
As part of its SIA project, each office did outreach to community partners. This strengthened relationships between the offices and other businesses and community organizations, and set the stage for ongoing and mutually beneficial collaborations.

The SIA experience promoted systems thinking on two different levels. It gave participants a clearer view of the role they played and the contributions they made within their workgroup, and of positive changes they could make to the way they functioned in the workplace. SIA also helped the participating offices understand their position relative to various external organizations, how those organizations impacted their workgroup, the circumstances and needs they shared with those organizations, and ways they could manage their interactions with those organizations to benefit both parties.

\section{Facilitating factors.}

A number of factors were vital to the success of SIA in each of the three offices. One facilitating factor was the structure of the modified learning organization model. Specific elements of the model that served to move the SIA projects forward were the time and space SIA provided the offices to develop their projects; the involvement and contributions of the Program Manager and researcher; the accountability required of each SIA participant; and the strong focus on utilizing staff's personal strengths.

A second facilitating factor was supervisors' support of SIA. In committing to participate in SIA, supervisors agreed to grant the Program Manager and researcher access to their office and their staff; facilitate SIA meetings and oversee their office's SIA project between meetings; assure all staff had a voice in the process; participate in SIA discussions and decision-making; volunteer to complete one or more SIA-related 
tasks; and be interviewed by the researcher at the completion of the SIA project. In fulfilling these obligations, the supervisors modeled behaviors conducive to learning organization processes for their staff.

Growing connections among staff were a third factor that facilitated SIA's success. In each office, new coworker relationships were formed and existing relationships were strengthened as the SIA projects evolved. These relationships contributed directly to the aforementioned improvements in staff communication, teamwork, camaraderie and morale.

Connections that developed between staff and community partners were a fourth factor that facilitated SIA. The frequency and quality of interactions between VR staff and staff in selected organizations and businesses improved noticeably during the SIA projects. These connections created a climate that allowed both VR and community partners to share useful information, begin collaborating in areas of mutual interest, and develop confidence that the partnerships initiated during SIA could be sustained and strengthened over time.

A fifth factor supporting SIA's success was staff's sense of ownership of their office's SIA project. It began to develop when each staff member was advised - verbally, and in writing - that participation in the study was voluntary, and each elected to participate. From that point forward, staff's sense of ownership gradually increased throughout SIA in all three participating offices. Staff's investment in their office's project appeared to be strengthened by supervisors' increasing support for staff 
ownership, having a strong voice in project-related decisions, collaborating with coworkers on SIA tasks, and interacting with community partners.

\section{Impeding factors.}

Some factors worked against SIA's success, at least in the initial stages of the offices' projects. One impeding factor was staff's dislike or distrust of their agency's headquarters. Some staff felt that headquarters' support for their offices' SIA project was inadequate; some questioned headquarters' motives for supporting SIA; some felt coerced by headquarters to participate in SIA; and some felt any gains made by efforts made during SIA would evaporate as soon as headquarters developed a new "pet" project. In each office, as the chosen SIA project evolved, staff focused less and less on headquarters' role in SIA, and more on their own SIA-related efforts and experiences.

Certain aspects of the existing climate and culture in the participating offices comprised a second impeding factor. These included staff conflicts that pre-dated SIA, and impacted the SIA process; the resistance of certain staff to even minor changes in work processes; and the agency's continuing focus on individual caseloads and performance measures, rather than teamwork and team rewards.

A third impeding factor was the reluctance of certain staff to fully engage in SIA. The causes for their lack of active participation included the resistance to change mentioned above, lack of motivation for team activities, discomfort in dealing with the public, desire for more supervisory direction, and doubts about SIA project sustainability.

The fourth and final impeding factor was work pressures and time constraints. The heavy workloads participants carried during SIA often drew staff's attention away 
from SIA-related tasks. Some participants believed, and stated, that the timeline of their office's SIA project was too short to accomplish the SIA goal their office had established.

\section{The economy.}

The SIA projects in the three participating offices began and ended in the midst of the Great Recession. I assumed at the outset of SIA that the effects of the dramatic financial downturn on the offices participating in the study would be negative in nature. The recession clearly did impact the offices overall, and the offices' SIA processes in particular, in negative ways. However, as the study progressed, I was surprised to discover that the recession also had an "upside" in the view of study participants.

As noted in Chapter 5 (Results), at the time of the study, VR staff were faced with a hiring freeze, payroll cuts, furloughs, and restrictions on travel and trainings. (These cutbacks were directly connected to state budget issues caused by the recession, and affected all human service workers employed by the state.) At the same time, VR staff's daily work was challenging because their clients - always at a disadvantage in the workplace due to their various disabilities - were facing progressively stiffer competition from non-disabled individuals as the unemployment rate rose, and were having a harder time meeting basic needs such as food, housing, and medical care.

Simply stated, the most negative impact the recession had on SIA was that staff in participating offices - faced with increasing caseloads, an indefinite hiring freeze, and clients who were themselves struggling in a tough economy - were burdened by heavy workloads. This limited the time staff had available to devote to their office's SIA project, regardless of their enthusiasm for or investment in the project. 
However, during SIA, some positive impacts of the recession emerged. Staff in Office B and Office $\mathrm{C}$ discovered that other human service organizations serving some of VR's clients were dealing with limitations similar to those VR was facing. This made the other organizations more - not less - willing to consider collaborations with VR that would stretch limited resources and better serve common clients. Office A discovered that employers were more interested than they had previously been in learning about assistance VR could provide if they hired VR clients: stipends during a new employee's probationary period, on-site mentors for new employees, technical assistance if special equipment was required, and training for company staff.

\section{Connection of findings to literature and theory.}

The literature suggests that primary strengths of learning organization models are that staff are given a strong voice in a team-based work process; the iterative learning organization process encourages meaningful communication between team members; and increased communication and collaboration lead to stronger relationships among coworkers (Annie E. Casey Foundation Casey Family Services, n.d.; Beddoe, 2009; Giesecke \& McNeil, 2004; Gustavsson, 2009; Khasawneh, 2011). The modified learning organization model implemented during SIA was designed to facilitate team-based projects and encourage staff voice, and evidence indicates it was successful on both counts. In addition, observations and participant reports indicate that the amount and quality of staff communication increased, and staff relationships were strengthened, during SIA. 
The literature also suggests that learning organization models assist staff in building working relationships with external partners, and developing more efficient work processes (Austin \& Hopkins, 2004; Gustavsson, 2009; Lewis et al., 2001). These benefits were clearly seen during SIA. In each of the three participating offices, staff made new connections - or strengthened existing connections - with community partners. As a result, working relationships between VR and those partners improved, more effective ways of working together were developed, and both VR and the partners saw benefits for staff, clients, and their organizations as a whole.

Another important benefit of learning organization models presented in the literature is that they create the opportunity for both individual and team learning. The positive effects of such learning include greater staff expertise, expanded team knowledge, individual and team pride in accomplishment, improved staff morale, and the ability of workgroups and organizations to be more flexible in adapting to changes in the workplace (Annie E. Casey Foundation Casey Family Services, n.d.; Beddoe, 2009; Giesecke \& McNiel, 2004; Gustavsson, 2009; Herman, 2007; Khasawneh, 2011; Schorr, 1997; Senge, 2001; Somunoglu, 2012).

During SIA, each of these effects were observed and reported as staff worked on the offices' chosen projects. Individual learning was in evidence as individuals assumed new roles, such as chair of a project-related committee or liaison to an external organization; took on new tasks, such as planning a group presentation, creating brochures, or contacting area employers; and increased their awareness of their abilities and interests and those of their coworkers. Team learning was in evidence as coworkers 
collaborated to develop and implement the office project they had chosen, met regularly to share information and revise their processes, and collaborated with community partners to develop new ways to improve client services and adapt to VR's recent removal from the federal Order of Selection. All of these efforts fostered individual and workgroup pride, a growing sense of camaraderie, and an increase in office morale.

Prior research indicates that certain issues tend to surface when organizations or workgroups attempt to implement learning organization models. These issues include individuals who cannot or will not give up their "expert" or "leader" role; individuals who cannot find their voice in the process, as they are unable to break their pattern of deferring to superiors; individuals who lack engagement in the process for personal reasons; and lack of trust between process participants and organization leaders (Baldwin, 2008; Lindsey \& Meredith, 2012; Nutting, Crabtree, \& Mcdaniel, 2012; Ruffolo, Kuhn, \& Evans, 2006; Somunoglu, Erdam, \& Erdam, 2012). During SIA, each of the participating supervisors admitted they had some difficulty moving from their daily role as office "leader" to their role as SIA facilitator, although they ultimately saw advantages in doing so. In each office, there were a few staff who were less engaged in SIA than their coworkers at the outset, or for the duration of the office's SIA project. Though their lack of engagement was easily observed, it was not always possible to discern whether it was due to deference to superiors, a general resistance to change, other personal reasons, or a combination of factors. SIA participants' lack of trust in their organization's headquarters, and their concern about headquarters' motives for supporting SIA, were evident in the early days of each SIA project. However, as previously noted, as SIA 
continued and staff began to develop a sense of ownership in their offices' project, concerns about management and its motives dissipated.

The literature offers advice and cautions to those considering the implementation of a learning organization model in a human service setting. It underscores the importance of on-site learning processes, rather than top-down training away from the workplace; launching a pilot program first, before attempting to implement a learning organization model throughout an organization; and having a clearly-defined and wellmanaged learning organization process. It also recommends strong supervisor and manager support throughout the learning organization process, the consistent encouragement of staff input throughout the process, and the involvement of any internal or external partners who will be impacted by organization changes in the process. In addition, it emphasizes the importance of building sufficient time into employees' workloads so they have opportunity to engage in a meaningful way in the learning organization process (Annie E. Casey Foundation Casey Family Services, n.d.; Baldwin, 2008; Beddoe, 2009; Davidson \& McMahon, 1999; Giesecke \& McNeil, 2004; Gray \& Schubert, 2012; Gustavsson, 2009; Herman, 2007; Hunter-Johnson \& Closson, 2012; Lindberg \& Meredith, 2012; Maden, 2011).

In addition, the literature speaks to the myriad of difficulties faced by human service workers functioning within top-down bureaucracies, and the benefits of allowing and encouraging workers to change their organizations from the bottom up. In Streetlevel Bureaucracy: The Dilemmas of Individuals in Public Service, Lipsky (2010) posits that public service workers - including social workers, health care workers, teachers, and 
law enforcement officers - are really policymakers, with considerable discretion and a strong day-to-day impact on their clients. However, they also have large caseloads and limited resources, and face a constant dilemma: trying to adhere to bureaucratic routines and procedures designed to treat all clients equally, while at the same time responding to unique, individual circumstances.

In Systems Thinking in the Public Sector, Seddon (2008) argues that clients are public-sector workers' stakeholders, and the workers need freedom from top-down control in order to be innovative and do the best for those stakeholders. He believes organizations should dispense with top-down compliance measures, and only assess the steps lower-level managers and staff choose to take to improve their work. In Seddon's view, this would result in more innovation, more engaged workers, and better-served clients.

In large part, SIA met the criteria for learning organizations outlined in the literature. It was conducted on-site in three VR offices, a team-based rather than topdown learning experience. It was a pilot program at a small number of sites, and not an organization-wide effort. The modified learning organization model was clearly defined for all participants, was identical in both content and process at all three sites, and was effectively managed at each site by the supervisor and the Program Manager. In their role as facilitators, the three participating supervisors were consistently supportive of SIA, and encouraged staff's participation in SIA-related activities throughout the process. Involvement with external partners was extensive, as it was the focus of each office's SIA project. There was less involvement with internal partners, although the director and 
staff at the agency's headquarters were provided regular SIA project updates, and responded to periodic requests from the participating offices for specific project-related resources throughout SIA.

In two areas, the SIA experience fell short of the vision presented in the literature. The first area was management support. The VR director and top managers authorized SIA, and provided some project-related resources, but they were not (with the exception of the Program Manager) involved in an ongoing way with the participating offices during the SIA projects. Some participants voiced their disappointment that management failed to support their office with their presence or regular contact during SIA, or to recognize and reward what their office accomplished during SIA in any meaningful way.

The second area was building sufficient time into employees' workloads to focus on the learning organization process. Although the agency's headquarters supported the participating offices' involvement in SIA, no work responsibilities were decreased or removed for supervisors or staff during SIA. As a result, with heavy workloads a daily reality, participants often had difficulty finding time to complete SIA-related tasks. Staff in each participating office frequently voiced their frustration about this situation; the essence of their comments was that the efforts they made during SIA were worthwhile, but they could have accomplished even more if some regular responsibilities had been "taken off the plate" during SIA.

Regarding the benefits of bottom-up organizational efforts, the SIA results showed that supervisors and staff in small workgroups, when granted latitude to assess their office's needs and take action to meet those needs, can find innovative and locale- 
specific ways to improve processes that benefit the workgroups, individual staff, and agency clients. The results also demonstrated that staff involvement in bottom-up efforts fosters a variety of positive developments, including improved staff communication, increased office morale, stronger connections between workgroups and community partners, and better services for clients.

As SIA was a pilot program which involved only three VR offices, it is not possible to speak to VR's experience in implementing SIA organization-wide. However, given the substantial investment of time and energy that the supervisor and staff in each office made in their relatively brief SIA projects, it is evident that growing and sustaining such a model organization-wide would be a major undertaking that would require a large and ongoing commitment of time, energy, and resources at all levels of the organization.

\section{Implications}

\section{Practice implications.}

What lessons can SIA offer to human service organizations interested in implementing a learning organization model? First and foremost, the study shows that such a model can be beneficial in a human service setting. It provides a clear process to use when involved in group efforts, and opportunities for individuals to learn and develop specific skills. It also creates an environment where co-workers become more cognizant of one another's strengths and skills, develop stronger relationships, and communicate more effectively. These developments lead to more efficient and effective team efforts, improved office morale, and new or renewed connections with community partners. 
Taken together, the benefits noted above can serve to move a workgroup away from a mode of individual workers reactively handling individual cases, and toward a mode of proactive collective problem-solving. Although the shift will not happen quickly or completely, the learning organization process - once experienced and integrated - can be utilized by a workgroup again and again.

Second, the SIA experience underscores that several elements are key to successful implementation of a learning organization model in a human service setting. These elements include: a well-structured and easily understood learning organization model, the voluntary involvement of all participants, strong and consistent supervisor support, and "outsiders" (such as mentors and researchers) involved in the learning organization process. Other crucial elements are increasing connection and collaboration among co-workers, and between staff and community partners. During SIA, as these relationships grew stronger, participants' commitment to the learning organization process, and sense of ownership in their office's SIA project, also grew stronger.

Third, the study suggests that if a human service organization wants to derive maximum benefit from implementation of a learning organization model, it would be wise to take participating workgroups' existing culture into account when planning and implementing the model, and to ensure top management's ongoing support of, and involvement in, the process. In addition, if at all possible, the organization should authorize and arrange temporary workload reductions for participating staff, to provide them the best opportunity to create and integrate a productive and meaningful learning organization experience. 


\section{Policy implications.}

\section{Benefits of implementation in small workgroups.}

In many instances, for financial or policy reasons, the implementation of a learning organization throughout an entire human service organization may not be feasible. This should not deter managers in human service organizations from encouraging and strongly supporting the implementation of a learning organization model in small workgroups within the organization.

VR is a clear example of a human service organization that was not in a position to consider the implementation of a learning organization model organization-wide, due to both financial constraints and ongoing organizational changes. The SIA study provides strong evidence that a learning organization model can have real and lasting benefits for small workgroups within human service organizations, whether or not the model is ever disseminated organization-wide. It also provides evidence that specific workgroups can reap benefits even if the learning organization model is modified in response to existing limitations; the learning organization effort does not receive strong and consistent management support; the organization does not commit large sums of money to the effort; the implementation of the learning organization model is time-limited; staff are burdened with heavy workloads during the learning organization process; staff distrust management going into the process; staff distrust one another going into the process; or certain staff are not fully engaged in the learning organization process.

If a learning organization model is successfully implemented in one segment of an organization, it will serve some workers and workgroups - and, by extension, the 
organization - well. If the organization should then elect to extend the model, the experience of the small workgroups will serve as a guide for the larger implementation effort.

\section{Importance of employee voice.}

As I noted in Chapter 3 (Process), I have long believed in the importance of giving staff in human service organizations voice, and the opportunity to do meaningful work with others. In my opinion, those that do not staff voice or value their input often make poor use of their employee's energy, creativity, talents and interests.

My observations and reflections during SIA shifted my outlook on the concept of voice. Prior to SIA, I had always thought first of the benefits of voice for individual employees - respect, increased confidence, and personal growth among them - and only afterwards did I consider the benefits for the employees' workgroups or organizations.

During SIA, I realized that giving staff voice - assuring they are seen and heard, and that their ideas and opinions are taken into account when decisions are made - is the critical first step from which a variety of positive developments for both individuals and workgroups naturally flow. Benefits for individual staff members and benefits for workgroups are not easily separated; the two categories exist in the same time and space, and draw from one another. Below, I offer a brief description of how this process played out in the SIA study.

SIA was purposely structured so that all participants would have voice and choice in their office's SIA project. Initially, some staff doubted this was true, and thought management must have ulterior motives for supporting SIA. However, once staff in each 
office realized that their voice actually counted in SIA, and they would have a legitimate say in what their office's project would be and how it would be developed, their sense of empowerment grew. This encouraged them to invest themselves in crafting an SIA project that suited them and their office, and this served to move the projects forward.

Once staff had invested time and energy in the development of their office's SIA project, they began to feel ownership of the effort. All participants had the freedom to decide what part they wanted to play in, and what contributions they wanted to make to, the SIA project, and this served to increase their commitment to the SIA project.

When individuals put their ideas, opinions, and effort into their office's SIA project, this allowed their co-workers to see their strengths and talents more clearly. Recognition of individual contributions increased the self-esteem and confidence of specific workers, and also gave rise to stronger working and personal relationships between co-workers.

As the SIA projects evolved in each office, staff realized that collective action taken either with co-workers or with staff in other organizations - could help facilitate their individual work goals. Working more closely with others, and learning more about their strengths and skills, they felt better able to make good use of co-worker's expertise. Their growing sense of "strength in numbers" increased camaraderie and improved office morale. It also served to increase their commitment to the SIA project, and to future team projects utilizing a learning organization model.

In the end, I was left with a strong sense that creating an environment that supports staff in giving voice to their opinions and ideas is the best and most cost- 
effective investment a human service workgroup or organization can make. SIA is proof that, even in less-than-optimal settings where resources are limited, workloads are heavy, changes are occurring, and office dynamics leave much to be desired, bringing staff together and encouraging their voice will reap many rewards for individual staff members, the workgroup, and the organization as a whole.

\section{My Role as Researcher}

I approached the SIA study fully aware that my presence as an observer in all of the SIA meetings and at other SIA-related activities in the participating offices would have a reactive effect. However, I had no way to know just what the effect would be, how I would respond to it, or whether the effect would be different in different offices.

When I first visited the participating offices to explain the study, I felt I had entrée because I arrived with the Program Manager, who is known - and well-respected - in all three offices. In addition, staff in each office were aware that the Program Manager and I had been authorized and encouraged by the agency's director to proceed with the study. However, as noted earlier in this report, being affiliated with headquarters was a mixed blessing; some staff disliked or distrusted headquarters' staff, or disagreed with specific organizational policies. Some were suspicious of headquarters' support for SIA and, by extension, distrustful of both me and the Program Manager.

I decided to respond to the initial polite-if-not-quite-warm welcome I received in each of the three offices by being clear and direct with study-related information, calm in response to "edgy" questions from some staff, and friendly but not over-eager. In the course of those first meetings, I explained that I worked for the Research Division, not 
VR, and that the study was connected to my academic program at Portland State. I also described the role that I would assume during the study - an overt observer of SIA meetings and activities, but not an active participant in the SIA process.

After each of the initial meetings, I debriefed with the Program Manager about the reactions of the supervisor and staff, contemplated the meeting and the Program Manager's perceptions, and then recorded my thoughts and feelings about my interactions with participants in my field notes. Both the debriefing and the incorporation of my reactions in the field notes became my standard practice.

The pause between the initial informational all-staff SIA meeting and the first "working" meeting in each office gave me time to reflect on, and make an important decision about, my role as researcher. First, I realized I did not want to be completely detached from the SIA process, but did want to sharply limit my impact on discussions related to the SIA projects. The goal of the SIA study was to encourage staff to learn to make decisions and take actions as a team, and I was not part of that team. At the same time, I wanted to be able to engage with staff, both so they would become more comfortable with my presence, and because I wanted a clear understanding of what was sure to be a complex and layered process.

After reflection, consultation with two colleagues, and review of qualitative research literature (Babbie, 1990; Berg, 2007;; Padgett, 2008; Patton, 1990), I decided it would be acceptable to share specific information about the SIA process, overviews of the policies or practices of other agencies, contact information that would move staff forward on the office's SIA project, or make occasional general comments or inquiries 
during SIA meetings. It would also be acceptable to have "water cooler" conversations with staff before and after meetings, as long as I did not quote any part of those conversations without specific permission. However, I would not be party to any decision-making about the SIA projects (although I certainly had opinions I would have liked to share).

By the time I had attended two or three SIA meetings in each office, the staff were visibly more relaxed. They seemed much less conscious of my presence during meetings; at the same time, they were much more likely to engage with me before and after meetings. Sometimes it was about SIA matters, but just as often the topics were very general. By then, I had shared with some staff that I had worked in a variety of different social service agencies. I was struck by how often the conversation turned to a staff member's frustration with their work situation. The concerns they raised paralleled many discussed in Lipsky's (2010) work on street-level bureaucrats: lack of resources, daunting and ever-changing agency rules and regulations, unrealistic work "targets," demanding clients, and deserving clients absent needed resources. But my conversations with staff were by no means consistently sober; they were just as often about kids, dogs, work-outs, or the upcoming staff picnic. I recognized I had begun to relax, too.

In short, the tone of the interactions between me, the SIA participants, and the Program Manager in and around SIA meetings lightened considerably as the SIA projects progressed in all three offices. That said, there were discernible differences among the offices; the staff in Office $\mathrm{C}$, who were the smallest group and had worked together the longest, were the most comfortable interacting with me, the Program Manager, and one 
another throughout SIA. The staff in Office A were very efficient and pleasant in their interactions with me, the Program Manager, and their supervisor, but not as engaged with one another. Overall, the staff in Office B were less animated and less effusive than staff in the other two offices. However, it was in Office B that several individuals approached me and asked for my help in planning future staff projects.

The Program Manager and I were closely connected in participants' minds; they saw us as a team of two. Even though we assumed different - and clearly delineated roles during the SIA project, we often arrived at and departed from SIA meetings together; we were both affiliated with headquarters, and contacted headquarters staff on participants' behalf throughout SIA; and we shared the responsibilities of introducing the modified learning organization model to staff in each office. Because of this, it is difficult to separate the influence our two roles might have had on study findings. For that reason, I will refer to the Program Manager and me as "we" in the following paragraph.

What impact did the roles we assumed during SIA have on the study findings? As noted in Chapter 3, Program and researcher contributions, we were told by some participants that having "new blood" in the office energized the SIA process, and that having "outsiders" participate in their office's SIA project made the project more important in their mind. Others suggested that our presence had the effect of keeping a strong focus on the SIA project, and made staff more accountable to fulfill SIA-related promises. Still others thanked us for providing information and accessing resources that helped them to reach their office's goal. Perhaps most surprising, during the study and in the interviews, several individuals thanked me for listening to their stories and their 
concerns because, they said, they are rarely given the chance to speak about the joys and trials of their daily work. All of this input suggests our presence played a very real role in making the SIA projects seem interesting and worthwhile to staff, and keeping the SIA projects in each office on track and moving forward.

\section{Research Limitations}

The research is best understood in the context of several limitations. First, as this was primarily a qualitative study, the results are not generalizable. Examining the experience of implementing a modified learning organization model in three small offices of a vocational rehabilitation organization at a specific point in time does not establish that other human service organizations, or even other offices within the same organization, would have a similar experience. The research findings can only suggest possible outcomes in other environments.

Another issue impacting generalizability is the fact that, from beginning to end, I was the only researcher conducting the SIA study. As discussed in Chapter 3, Process, I came to this study with particular ideas, beliefs, skills, and experiences that would inevitably influence my approach to, and my understanding of, this research. This can be viewed as a limitation, as it is not possible to know if the study would have evolved in a similar way if another researcher had conducted the study, or if additional researchers had been involved.

The second limitation, specific to the quantitative findings, is the small sample size. The total number of staff participating in SIA was less than 40. This may have made 
it more difficult to find statistically significant relationships when comparing the responses of staff in Offices A, B and C to the closed-ended online survey questions.

The impact of race/ethnicity, gender, and age on participants' SIA experiences was not examined, and this is a third limitation of the research. The research design did not address differences between the engagement, actions, and perceptions of white SIA participants (a majority) and non-white SIA participants (a minority); female SIA participants (a majority) and male SIA participants (a minority); or middle-aged participants (a majority) and younger participants (a minority). Nor did it address how participants' race/ethnicity, gender and age impacted their interactions and communications with the supervisor, Program Manager and me during SIA. Although such information would have been of interest and value, it was beyond the scope of this research. Given the small number of SIA participants, questions about race/ethnicity, gender, and age could not be asked in the online survey without compromising promised confidentiality; given the resources available for the study, interviewing participants at the completion of their office's SIA project was not feasible.

A fourth limitation is the lack of client voice in the research, even though it was clear from the beginning of SIA that the projects the participating offices chose to develop and implement were created with better client service in mind. The lack of client input into the research was due to two factors. The first factor was that the vulnerability of many VR clients made obtaining IRB permission to interview or survey those clients a complex and time-consuming process, which I elected to forgo. The second factor was the relatively short time the research design granted the offices to develop and implement 
their SIA projects, which meant few clients would have directly experienced the benefits or drawbacks of any changes made by the time the SIA projects were completed.

Social desirability (the tendency of respondents to reply in a manner that will make them seem close to "average" and be viewed favorably by others) is widely recognized as a limitation of qualitative research. I attempted to mitigate the effect of social desirability in several ways. First, I developed an anonymous, self-administered online survey for SIA participants, as such surveys are thought to mitigate social desirability by providing respondents a reassuring sense of neutrality and detachment. Second, at the beginning of each interview, I reviewed the Informed Consent with the interviewee, emphasizing confidentiality measures. Third, I included questions about SIA's impact on the office and on VR clients, as focusing on non-personal issues also tends to mitigate social desirability.

Reactivity (the potentially distorting effects of the researcher's presence on participants' beliefs and behaviors) is also considered a limitation of qualitative research. As indicated in My Role as Researcher, above, I made the following efforts to mitigate reactivity in the SIA study. First, I chose to be overt in my role as a researcher, but not to be an active participant in SIA decision-making processes, which served to limit my influence. Second, I had prolonged engagement with the SIA participants, which is known to mitigate reactivity. As participants became increasingly engaged in their office's SIA projects, their awareness of my presence - and thus the potential for reactivity - decreased. Third, I took detailed field notes throughout the survey, including direct quotes, and gathered interview and survey data for the purpose of triangulation. 
Finally, I endeavored to strike a balance between being present, engaged, and aware during SIA meetings and activities, and retaining some analytic distance.

As discussed in Chapter 2 (Literature Review), learning organization theory evolved in corporate settings, and has only recently emerged as a subject of serious study in human service settings. As a result, there is a notable lack of research on implementation of learning organization models in such settings, which effectively leaves organizations interested in experimenting with learning organization concepts without a road map (Austin \& Hopkins, 2004; Davidson \& McMahon, 1999; Lewis et al., 2001). Without parameters set by a body of previous research, SIA was, of necessity, an exploratory rather than an explanatory study. This could be regarded as the fifth limitation of this research. Alternately, SIA could be viewed as one piece of the groundwork that will create a foundation for future research in this area.

\section{Directions for Future Research}

The SIA experience shed light on several areas of inquiry that, although not within the purview of this study, are clearly deserving of further research. These areas include: examination of the impact of participants' race/ethnicity, gender, and age on their learning organization experiences; examination of the effect of power differentials on learning organization processes; examination of the impact of learning organization processes on clients in human service settings; and examination of the factors that would assist organizations in sustaining gains made during learning organization processes. Research in these areas would require attention to literature and theories not addressed in the SIA study, as well as further data collection and analysis. 


\section{Race/ethnicity, gender and age.}

As noted in "Research Limitations," above, the SIA study, with its small and quite homogeneous group of participants, was not designed to address the impact of participants' race/ethnicity, gender, or age on their SIA experience. That being said, if SIA were expanded to include more - or all - VR offices, or if a learning organization model was implemented more broadly in another human service organization, it would be very important to discern if different or more diverse groups experienced the learning organization process differently. Only in this way could researchers and organization leaders come to a clear understanding of the strengths and limitations of utilizing a learning organization model in a specific organizational setting. Fortunately, there is a large body of extant literature regarding the experience of employees of different races/ethnicities, genders, and ages in the workplace. This literature would serve as a strong guide for those electing to design studies that explore the myriad ways that learning organization processes can impact specific groups of workers.

\section{Power differentials.}

As discussed in Chapter 2 (Literature Review), the primary criticism of learning organization theory is that it fails to take into account the power differentials that are intrinsic to organizational life. Critics posit that even in situations where management "grants" workers a voice in particular processes, power is not equalized in any real or lasting way. This is because workers have internal controls, peers in workgroups impose controls, and the existing organizational culture strongly conveys (with, or without, words) how it is "supposed to be" (Beddoe, 2009; Caldwell, 2011). 
During the SIA study, it was observed and reported that the participating supervisors became more open to sharing power with their staff, and that this was critical to the successful implementation of the modified learning organization model. However, the way in which power relationships - supervisor-staff, management-staff, staff-staff, and others - affect learning organization efforts is worthy of further investigation. Researchers interested in pursuing this avenue of inquiry would be informed by power theories put forward by Lukes (2004), French and Raven (1959), and others, as well as by the growing body of literature on power in the workplace.

\section{Clients.}

As explained in "Research Limitations," above, VR clients did not have a voice in the SIA study, beyond decisions staff made during each SIA project with the best interest of their clients in mind. More direct client input was lacking, both because obtaining IRB permission to interact with vulnerable clients was problematic, and because the short length of the SIA projects left little time for project effects to filter down to clients.

The mission of human service organizations is to provide the best possible service to clients. Thus, if such an organization chooses to implement a learning organization model, a logical next step would be to examine the impact the implementation has on the organization's clients. However, a comprehensive understanding of the impact on clients would only be possible if the learning organization model were disseminated throughout the organization, and if the model were in active use for an extended period of time. Research on client impact would likely be a complex and time-consuming process, but well worth the effort in an organization that exists to serve its clients well. 
Any study of a learning organization's impact on its clients would be well-served to take the concept of "parallel process" - previously discussed in Chapter 2 (Literature Review) - into account. This concept suggests that if employees feel supported within a strong and established learning culture they will be better able to help clients set and attain personal goals (Austin \& Hopkins, 2004; Bargal, 2000).

\section{Sustainability.}

The SIA study was of relatively short duration. It focused on the factors that facilitated and impeded the implementation of a modified learning model in three VR offices, SIA participants' experiences during the implementation process, and their perspectives on that experience. Within the space of a few months, clear benefits were observed and reported for supervisors, staff, and community partners involved in the study.

However, as Senge and his colleagues $(1990,1999)$ are quick to point out, fullfledged learning organizations can take years to develop to their full potential. A shortterm study such as SIA is not positioned to observe or record the full impact a learning organization model might have in a human service organization. Implementing a learning organization model - as in the SIA study - and sustaining such a model are two different efforts.

Research that examined a learning organization process as it matured would require more time and resources than SIA had at its disposal, and would also require engagement with theories such as diffusion of innovation (Gladwell, 2000; Rogers, 1995; 
Wheatley, 1992) and literature not included in SIA. This is an important area of inquiry that deserves to be addressed in future research.

\section{Summary}

The SIA study was a modest effort to document the implementation of a modified learning organization model in a human service setting. Three offices of a vocational rehabilitation organization (VR) were involved in SIA, and there were about 40 SIA participants overall. It took staff in each office between four and eight months to complete their SIA project; during this time, VR was negotiating a major organizational change and dealing with the effects of the Great Recession. Aside from the presence and support of one program manager from headquarters throughout SIA, VR management offered the offices little in terms of direct support or rewards. Supervisors and staff participating in SIA were not relieved of any of their normal work responsibilities while SIA was in operation.

Despite these constraints, the modified learning organization model was sufficiently robust to create some very positive effects in three office environments selected to participate in the study because of their differences. These effects included the development of stronger relationships among coworkers, improved staff communication, more effective teamwork, and improved office morale. Stronger connections were also developed between staff and specific community partners, which led to more efficient work processes for both parties and plans for future collaborations.

The SIA study shows that a learning organization model can be beneficial in a human service setting. It points to several factors that are key to successful 
implementation of a learning organization model: a well-structured model; voluntary participation of staff; strong supervisor support; involvement of "outsiders" in the process; and growing connections among staff, and between staff and community partners. It suggests that, to promote a successful learning organization experience, organizations should take the culture of existing workgroups into account, adjust employees' workloads to facilitate participation in the process, and provide strong management support.

The study makes clear that a learning organization model does not have to be implemented organization-wide in order to have value. Such models can help small workgroups - and individuals within those workgroups - attain knowledge and skills, and develop work processes, that will serve them, their community partners, and their clients well.

Finally, conditions do not have to be ideal for a learning organization model to be successfully implemented. SIA utilized a learning organization model modified to accommodate realities faced by the individuals and the organization involved in the study. The model was implemented in small workgroups, in the midst of an economic downturn, during a period of significant organizational change, with little backing from the larger organization. This study demonstrates that - even in an environment of limited resources, shifting circumstances, and scant external support - important positive changes can occur in workgroups or organizations that embrace a learning organization model. 


\section{References}

Annie E. Casey Foundation Casey Family Services (n.d.). Learning while doing in the human services sector: Becoming a learning organization through organizational change. New Haven, CT: Author.

Arrington, P. (2008). Stress at work: How do social workers cope? (NASW Membership Workforce Study.) Washington DC: National Association of Social Workers.

Ashmos, D. P., \& Duchon, D. (2000). Spirituality at work: A conceptualization and measure. Journal of Management Inquiry, 9, 134-145.

Austin, M. J. (2008). Strategies for transforming human service organizations into learning organizations: Knowledge management and the transfer of learning. Journal of Evidence-based Social Work, 5 (3/4), 569-596.

Austin, M. J., \& Hopkins, K. M. (Eds.) (2004). Supervision as collaboration in the human services: Building a learning culture. Thousand Oaks, CA: Sage.

Babbie, E. (1990). Survey research methods (2nd ed.). Belmont, CA: Wadsworth.

Baldwin, M. (2008). Promoting and managing innovation: Critical reflection, organizational learning and the development of innovative practice in a national children's voluntary organization. Qualitative Social Work, 7(3), 330-348.

Bandura, A. (1997). Self-efficacy: The exercise of control. NY: W. H. Freeman.

Bargal, D. (2000). The manager as leader. In R. J. Patti (Ed.), The handbook of social welfare management (pp. 303-320). Thousand Oaks, CA: Sage.

Beddoe, L. (2009). Creating continuous conversation: Social workers and learning organizations. Social Work Education, 28(7), 722-736. 
Bell, E., Robinson, A, \& See, C. (2013). Do written mandatory accreditation standards for residential care positively model learning organizations?: Textual and critical discourse analysis. International Journal of Nursing Studies, 50 (11), 1446-1458.

Bennett, S., \& Brown, J. (1995). Mindshift: Strategic dialogue for breakthrough thinking. In S. Chawla \& J. Renesh (Eds.), Learning organizations: Developing cultures for tomorrow's workplace (pp. 167-184). Portland, OR: Productivity Press.

Berg, B. L. (2007). Qualitative research methods for the social sciences (6th ed.).Boston, MA: Pearson/Allyn and Bacon.

Brody, R. (2005). Effectively managing human service organizations (3rd ed.). Thousand Oaks, CA: Sage.

Buckingham, M., \& Coffman, C. (1999). First, break all the rules: What the world's greatest managers do differently. NY: Simon \& Schuster.

Burud, S., \& Tumolo, M. (2004). Leveraging the new human capital: Adaptive strategies, results achieved, and stories of transformation. Palo Alto, CA: Davies-Black.

Caldwell, R. (2012). Leadership and learning: A critical reexamination of Senge's learning organization. Systemic Practice \& Action Research, 25, 39-55. doi:10.1007/s11213-011-9201-0.

Center for State \& Local Government Excellence (2010). Survey findings: The great recession and the state and local government workforce. Retrieved from http://www.slge.org/publications/the-great-recession-and-the-state-and-localgovernment workforce. 
Center for State \& Local Government Excellence (2014). Survey findings: State and local government workforce: 2014 trends. Retrieved from http://www.slge.org/publications/state-and-local-government-workforce-2014$\underline{\text { trends. }}$

Charmaz, K (2006). Constructing grounded theory: A practical guide through qualitative analysis. Thousand Oaks, CA: Sage.

Chawla, S., \& Renesh, J. (Eds.), (1995). Learning organizations: Developing cultures for tomorrow's workplace. Portland, OR: Productivity Press.

Chowdhury, S. (2000). Management 21C: Someday we'll all manage this way. London, U. K: Prentice Hall.

County Welfare Directors Association of California, \& California State Association of Counties (2009). Human services in a time of economic crisis: An examination of California's safety-net programs and related economic benefits for communities. Retrieved from www.cwda.org/uploads/CSAC-CWDA-Joint-repor0409.pdf.

Davidson, A. S., \& McMahon, R. R. (1999). One city's journey toward more responsive government. Popular Government, Winter 1999, 12-29.

Deal, T. E., \& Kennedy, A. A. (2000). Corporate cultures: The rites and rituals of corporate life. NY: Perseus Books Group.

DeVellis, R. F. (2003). Scale development: Theory and applications (2nd ed.). Thousand Oaks, CA: Sage.

Dillman, D. A. (1978). Mail and telephone surveys: The total design method. NY: John Wiley \& Sons. 
Division of Vocational Rehabilitation, Washington State Department of Social and Health Services (2010). Employ your abilities [Brochure]. Olympia, WA: Author.

Edwards, P., \& Wajcman, J. (2005). The politics of working life. Oxford, U. K.: Oxford University Press.

Farmbry, K. (2004). Environmental scanning for nonprofit human service organizations. In M. J. Austin \& K. M. Hopkins (Eds.), Supervision as collaboration in the human services (pp. 252-260). Thousand Oaks, CA: Sage.

Fenwick, T. J. (1998). Questioning the concept of the learning organization. In S. Scott, B. Spencer, \& A. Thomas (Eds.), Learning for life: Canadian readings in adult education (pp. 150-162). Toronto, Canada: Thompson.

Finnigan, K. D., \& Daly, A. J. (2012). Mind the gap: Organizational learning and improvement in an underperforming urban system. American Journal of Education, 119 (1), 41-71.

Fong, R., \& Furuto, S. (Eds.) (2001). Culturally competent practice: Skills, interventions, and evaluations. Boston, MA: Allyn and Bacon.

Fowler, F. J. (1995). Improving survey questions: Design and evaluation. Thousand Oaks, CA: Sage.

French, J., \& Raven, B. (1959). The bases of social power. In D. Cartwright (Ed.), Studies in social power (pp. 150-167). Ann Arbor, MI: Institute for Social Research.

Ganzer, C., \& Ornstein, E. D. (1999). Beyond parallel process; relational perspectives on field instruction. Clinical Social Work Journal, 20(3), 231-246. 
Giesecke, J., \& McNeil, B. (2004). Transitioning to the learning organization. Library Trends, 53(1), 54-68.

Gladwell, M. (2000). The tipping point: How little things can make a big difference. NY: Little Brown and Company.

Glisson, C. (2000). Organizational climate and culture. In R. Patti (Ed.), The handbook of social welfare management (pp. 195-218). Thousand Oaks, CA: Sage.

Goodman, C. J., \& Mance, F. M. (2011). Employment loss and the 2007-09 recession: An overview. Monthly Labor Review, April 2011. Retrieved from http://www.bls.gov/opub/mlr/2011/04/art1full.pdf.

Gray, M., \& Schubert, L. (2012). Sustainable social work: Modelling knowledge production, transfer, and evidence-based practice. International Journal of Socia Welfare, 21, 03-214. DOI: 10.1111/j.1468-2397.2011.00802.x.

Gustavsson, M. (2009). Facilitating expansive learning in a public sector organization. Studies in Continuing Education, 31(3), 245-259.

Gutierrez, L., GlenMaye, L., \& Delois, K. (1995). The organizational context of empowerment practice: Implications for social work administration. Social Work, 40(2), 249-257.

Hager, R. M. (2004). Order of selection for vocational rehabilitation services: An option for state VR agencies who cannot serve all eligible individuals. NY: Cornell University School of Industrial and Labor Relations Employment and Disability Institute. 
Hay, I. (2005). Qualitative research methods in human geography (2nd ed.). Oxford, U. K.: Oxford University Press.

Herman, D. V. (2007). Celebrating courage in the learning organization (response ability pathways). Reclaiming Children and Youth, 16(3), 50-53.

Hunter-Johnson, Y., \& Closson, R. (2012). Adult educators' perceptions of their organization promoting learning practices and culture: A Caribbean law enforcement context. Adult Learning, November 2012, 178-197. doi:10.1177/1045159512457919

Iglehart, A. P. (2000). Managing for diversity and empowerment in social services. In R.J. Patti (Ed.), The handbook of social welfare management (pp. 425-444). Thousand Oaks, CA: Sage.

Katz, D., \& Kahn, R. I. (1992). Organizations and the system concept. In J. M. Shafritz \& J. S. Ott (Eds.), Classics of organizational theory (3rd ed.; pp. 270-280). Pacific Grove, CA: Brooks/Cole.

Kaye, B., \& Jordan-Evans, S. (2005). Love 'em or lose 'em: Getting good people to stay. San Francisco, CA: Berrett-Koehler.

Khasawneh, S. (2011). Learning organization disciplines in higher education institutions: An approach to human resource development in Jordan. Innovative Higher Education, 36 (4), 273-285.

Kofman, F., \& Senge, P. (1995). Communities of commitment: The heart of learning organizations. In S. Chawla \& J. Renesh (Eds.), Learning organizations: 
Developing cultures for tomorrow's workplace (pp. 15-43). Portland, OR:

Productivity Press.

Kuhn, A. (1974). The logic of social systems: A unified deductive system based approach to social science. San Francisco, CA: Jossey-Bass.

Ladany, N., Constantine, M. G., Miller, K., Erickson, C. D., \& Muse-Burke, J. L. (2000). Supervisor countertransference: A qualitative investigation into its identification and description. Journal of Counseling Psychology, 47, 102-115.

Lawrence, P. A., \& Lorsch, J. W. (2001). Environmental adaption through differentiation and integration. In W. E. Natemeyer \& J. T. McMahon (Eds.), Classics of organizational behavior (3rd ed.; pp. 391-398). Prospect Heights, IL: Waveland Press.

Leana, C. R., \& Rousseau, D. M. (2000). Relational wealth: The advantages of stability in a changing economy. Oxford, U. K.: Oxford University Press.

Lewis, J. A., Lewis, M. D., Packard, T., \& Souflee, F. (2001). Management of human service programs (3rd ed.). Belmont, CA: Brooks/Cole Thomson Learning.

Lipsky, M. (2010). Street-level bureaucracy: Dilemmas of the individual in public services (30 ${ }^{\text {th }}$ Anniversary Expanded Edition). New York: Russell Sage Foundation.

Lindberg, A., \& Meredith, L. (2012). Building a culture of learning through organizational development: The experiences of the Marin County health and human services department. Journal of Evidence-Based Social Work, 9(1-2), 2742. 
Lincoln, Y. S., \& Guba, E. G. (1985) Naturalistic Inquiry. Newbury Park, CA: Sage. Lipshitz, R., \& Popper, M. (2000). Organizational learning in a hospital. Journal of Applied Behavioral Science, 36(3), 345-361.

Longhi, D., Wang. B., \& Felver, B. (2007). Vocational rehabilitation rates since 2000. Aftermath: To what degree is field office performance stable after the 2000 decline? Olympia, WA: State of Washington, Department of Social and Health Services, Research and Data Analysis Division.

Longhi, D., Wang, B., Felver, B., \& Adams, S. (2007). Vocational rehabilitation rates since 2000: A study of two cohorts. Examining Washington State's vocational rehabilitation rates: Why the decline? Olympia, WA: State of Washington, Department of Social and Health Services, Research and Data Analysis Division.

Lukes, S. (year). Power: A radical view (2nd ed.). Basingstoke, U.K.: Palgrave Macmillan.

Maden, C. (2012). Transforming public organizations into learning organizations: A conceptual model. Public Organization Review, 12, 71-84. doi: 10.1007/s11115011-0160-9

Marquardt, M. J. (2004). Building the learning organization (2nd ed.). Palo Alto, CA: Davies-Black.

Maynard, B. R. (2010). Social service organizations in the era of evidence-based practice: The learning organization as a guiding framework for bridging science to service. Journal of Social Work, 10 (3), 301-316. 
McKenna, E. (1994). Business psychology \& organizational behaviour: A student's handbook. Philadelphia, PA: Lawrence Erlbaum.

Menefee, D. (2004). The managerial roles of the supervisor. In M. J. Austin \& K. M. Hopkins (Eds.), Supervision as collaboration in the human services: Building a learning culture (pp. 137-150). Thousand Oaks, CA; Sage.

Merriam, S. B. (2001). Qualitative research and case study applications in education. San Francisco, CA: Jossey-Bass.

Mitroff, I. I., \& Denton, E. A. (1999). A study of spirituality in the workplace. Sloan Management Review, 40, 83-92.

Modaff, D. P., DeWine, S., \& Butler, J. (2011). Organizational communication:

Foundations, challenges, and misunderstandings ( $2^{\text {nd }}$ Ed.). Boston, MA: Pearson Education.

National Association of Social Workers (2009). Social work speaks: National Association of Social Worker's policy statements 2009-2012 (8th ed.). Washington, DC: NASW Press.

Nutting, P. A., Crabtree, B. F., \& Mcdaniel, R. R. (2012). Small primary care practices face four hurdles - including a physician-centric mind-set in becoming medical homes. Health Affairs, 31(11), 2417-2422.

Padgett, D. K. (2008). Qualitative methods in social work research (2nd ed.). Los Angeles, CA: Sage.

Patton, M. Q. (1990). Qualitative evaluation and research methods (2nd ed.). Newbury Park, CA: Sage. 
Pfeffer, J. (2007). Working alone: What ever happened to the idea of organizations as communities? In J. O’Toole, E. E. Lawler, \& Meisinger, S. R.(Eds), The new American workplace. NY: Palgrave Macmillan.

Pil, F. K., \& Leana, C. R. (2000). Free-agency vs. high-involvement approaches to skill development: Enhancing relational wealth. In C. R. Leana \& D. M. Rousseau

(Eds), Relational wealth: The advantages of stability in a changing economy (pp. 116126. Oxford, U. K.: Oxford University Press.

Poell, R. F. (2005). Learning organizations and communities of practice: A critical evaluation. In J. P. Wilson (Ed.), Human resource development: Learning and training for individuals and organizations (2nd ed.) (pp. 99-110). Sterling, VA: Kogan Page.

Rath, T. (2007). StrengthsFinder 2.0 . NY: Gallup Press.

Ravasi, D., \& Schultz, M. (2006). Responding to organizational identity threats: Exploring the role of organizational culture. Academy of Management Journal, 49(3), 433-458.

Reason, P., \& Bradbury, H. (2001). Handbook of action research. Thousand Oaks, CA: Sage.

Rogers, E. M. (1995). Diffusion of innovations. NY: The Free Press.

Rothstein, J., \& Valletta, R. G. (2014). Scraping by: Income and program participation after the loss of extended unemployment benefits (IRLE Working Paper No. 10114). Retrieved from http://irle.berkeley.edu/workingpapers/101-14.pdf. 
Rousseau, D. M. (n. d). Organizational climate and culture. In J. M. Stellman (Ed.), Encyclopaedia of occupational health \& safety (Macro-Organizational Factors). Retrieved from www.ilo.org/safework_bookshelf/english?content\&nd=857170438.

Royse, D. (1999). Research methods in social work (3rd ed.). Belmont, CA: Wadsworth/Thomson Learning.

Ruffolo, M. C., Kuhn, M. T., \& Evans, M. E. (2006). Developing a parent-professional team leadership model in group work: Work with families with children experiencing behavioral and emotional problems. Social Work, 51(1), 39-48.

Schein, E. H. (1992). Defining organizational culture. In J. M. \& J. S. Ott (Eds), Classics of organizational theory (3rd ed.; pp. 490-534). Pacific Grove, CA: Brooks/Cole.

Schneider, B., \& Smith, D. B. (2004). Personality and organizations. Mahwah, NJ: Lawrence Erlbaum.

Schneider, B., White, S. S., \& Paul, M. C. (1998). Linking service climate and customer perceptions of service quality: Test of a causal model. Journal of Applied Psychology, 83(2), 150-163.

Schon, D. (1973). Beyond the stable state. NY: Norton.

Schorr, L. B. (1997). Common purpose: Strengthening families and neighborhoods to rebuild America. NY: Anchor Books/Doubleday.

Schrater, D. (2008). The importance of understanding organizational culture. Retrieved from http://www.oregon.gov/DSH/vr/index/shtml. 
Senge, P. M. (1990). The fifth discipline: The art and practice of the learning organization. NY: Currency/Doubleday.

Senge, P. M. (2001). The learning organization. In W. E. Natemeyer \& J. T. McMahon (Eds.), Classics of organizational behavior (3rd ed.; pp. 468-472). Prospect Heights, IL: Waveland Press.

Senge, P. M. (2006). The fifth discipline: The art and practice of the learning organization. NY: Currency/Doubleday.

Senge, P. M., \& Kaufer, K. H. (2000). Communities of leaders or no leadership at all. In S. Chowdhury (Ed.), Management 21c: Someday we'll all manage this way (pp. 186-204). London. U. K.: Prentice Hall.

Senge, P., Kleiner, A., Roberts, C., Ross, R., Roth, G., \& Smith, B. (1999). The dance of change: The challenges to sustaining momentum in learning organizations. NY: Currency/Doubleday.

Senge, P. M., Kleiner, A., Roberts, C., Ross, R. B., \& Smith, B. J. (1994). The fifth discipline fieldbook: Strategies and tools for building a better learning organization. NY: Currency/Doubleday.

Shafritz, J. M., \& Ott, J. S. (Eds.). (1992). Classics of organizational theory (3rd ed.). Pacific Grove, CA: Brooks/Cole.

Sherman, S. G., Leahy, M. J., Del Valle, R., Anderson, C. A., Tansey, T. N., \& Lui, K. (2014). Organizational and cultural factors that promote creative best practices in the public rehabilitation program: Findings from a four-state multiple case study. Journal of Vocational Rehabilitation, 41(2), 115-125. 
Shulman, L. (1993). Interactional supervision. Washington, DC: National Association of Social Workers Press.

Singleton, R. A., \& Straits, B. C. (2005). Approaches to social research (4th ed.). NY: Oxford University Press.

Solomon, N. (2001) Workplace learning as a cultural technology. In T. Fenwick (Ed.), Sociocultural perspectives on learning through work [Special issue]. New Directions for Adult and Continuing Education, 92, 41-51.

Somunoglu, S., Erdem, E., \& Erdem, U. (2012). A study on determining the perception of learning organization applications by health sector workers. Journal of Medical Systems, 36(6), 3925- 31.

Stake, R. E. (2000). Case studies. In N. K. Denzin \& Y. S. Lincoln (Eds.), Handbook of qualitative research. Thousand Oaks, CA: Sage.

State of Washington Office of Financial Management (2014). 2013 data book: Human services. Retrieved from ofm.wa.gov/databook/pdf/human.pdf.

Steinmetz, A. M. (1991). Doing. In M. Ely, M. Anzul, T. Friedman, D. Garner, \& A. M. Steinmetz (Eds), Doing qualitative research: Circles within circles (pp. 41-68). London, U. K.: Falmer.

Stoddart, K. (1986). The presentation of everyday life. Urban Life, 15(1), 103-121.

Thompson, J. D. (1992). Organizations in action. In J. M. Shafritz \& J. S. Ott (Eds.), Classics of Organizational Theory (3rd ed.; pp. 281-293). Pacific Grove, CA: Brooks/Cole. 
U.S. Department of Commerce, Economics and Statistics Administration, U.S. Census Bureau (2014). 2012 census of governments: Employment summary report. Retrieved from www.census.gov/govs/apes.

U.S. Department of Labor, Bureau of Labor Statistics (2012). The recession of 20072009: BLS spotlight on statistics. Retrieved from http://www.bls.gov/spotlight/2012/recession/pdf/recession_bls_spotlight.pdf.

U.S. Department of Labor, Bureau of Labor Statistics (2014a). A cohort component analysis of the 2007-2009 recession. Monthly Labor Review, March 2014. Retrieved from http://www.bls.gov/opub/mlr/2014/article/a-cohort-componentanalysis-of-the-2007-009-recession.htm.

U.S. Department of Labor, Bureau of Labor Statistics (2014b). Charting the labor market: Data from the current population survey (CPS). Retrieved from http://www.bls.gov/empsit/cps_charts.pdf.

U.S. Government Accountability Office (2012a). Temporary Assistance for Needy Families: Update on program performance (GAO-12-812T). Retrieved from Www.gao.gov/products/GAO-12-812T.

U.S. Government Accountability Office (2012b).Unemployment insurance: Economic circumstances of individuals who exhausted benefits (GAO-12-408). Retrieved from www.gao.gov/products/GAO-12-408.

U.S. Government Accountability Office (2013). State and local governments' fiscal outlook: April 2013 update. Retrieved from www.gao.gov/assets/660/654255/pdf. 
Van Buren, H. J., \& Leana, C. R. (2000). Building relational wealth through employment practices: The role of organizational social capital. In C. R. Leana \& D. M. Rousseau (Eds.), Relational wealth: The advantages of stability in a changing economy (pp. 233-244.). Oxford, U. K.: Oxford University Press.

Walonick, D. S. (1993). General systems theory. Retrieved from www.statpac.org/Walonick/systems-theory.htm.

Wheatley, M. J. (1992). Leadership and the new science. San Francisco, CA: BerrettKoehler.

Whitaker, T., Weismiller, T., \& Clark, E. (2006). Assuring the sufficiency of a frontline workforce: A national study of licensed social workers. Washington, DC: National Association of Social Workers.

Yin, R. K. (2003). Case study research (3rd ed.). Thousand Oaks, CA: Sage. 
Appendix A - Learning from Offices Study Overview 
Core Practices and Values

Core practices and values

And their influence

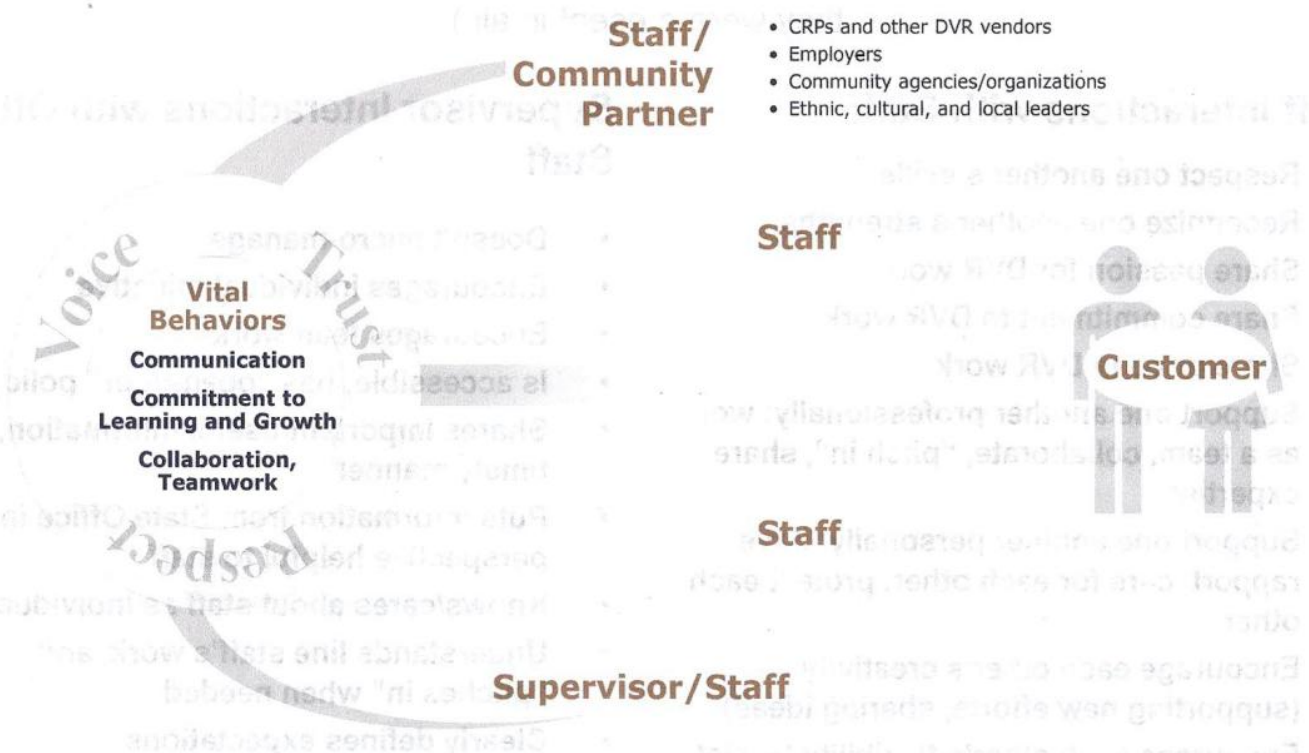

Note: This model summarizes relationships learned and observed during a study of 5 DVR Field Offices that included 44 individual interviews, many focus group meetings, and case studies. The purpose of the study was to learn more about what influences customer success. The following pages provide more details. 
List of Attributes Learned/Observed

\section{List of Attributes Learned/Observed}

(Please note: The strength of these attributes varies in the five office visited, however they were present in all.)

\section{Staff Interactions with Customers}

(When Community Partners were directly involved, those interactions contributed to some of these attributes with customers, e.g. communication, expectations, etc.)

- Welcoming environment

- Positive, hopeful attitude

- Direct, honest communication

- Clear goals, expectations

- Customers challenged to learn and grow

- Customers given a strong "voice"; empowered to make choices

- Customers encouraged to be actively invested in DVR process

- Options created for customers, using creativity and flexibility

- Staff didn't give up on customers

- Staff knew when to close a case (customer not a "fit" for DVR)

- Staff knew when to "pause" a case (customer not ready for DVR services)

\section{Staff Interactions with Community}

Partners (Community Partners includes CRPs, other vendors, employers, agencies, schools, hospitals/clinics, ethnic/cultural organizations, etc.)

- Focus on community partners (strong priority)

- Long-standing connections with various community partners

- $\quad$ Strong working relationships with community partners ("We're in this together")

- Regular meetings with community partners (re: individual cases, or general issues or interests)

- Supervisor supports community outreach, enables staff to create and maintain community partnerships

- Supervisor participates in community outreach (collaborates with other agencies for welfare of DVR clients in general) 
Vital Behaviors for Customer Success

\section{Vital Behaviors for Customer Success}

Many of these behaviors apply to all of the relationships observed. Items that are checked received highest votes by supervisors reviewing the study results.

\section{Communication \\ $\checkmark$ Open-ended (free flow of information, open- door policy) \\ $\checkmark$ Solicit input (empower by giving "voice") \\ $\checkmark$ Clearly outline expectations \\ - Lateral (not top-down) \\ - Talk frequently (keep \\ talking, don't give up) \\ - Active listening (really hear what is being said) \\ - Share information directly and honestly (tell it like it is) \\ - Supervisor viewed as liaison to SO, not holder of wisdom}

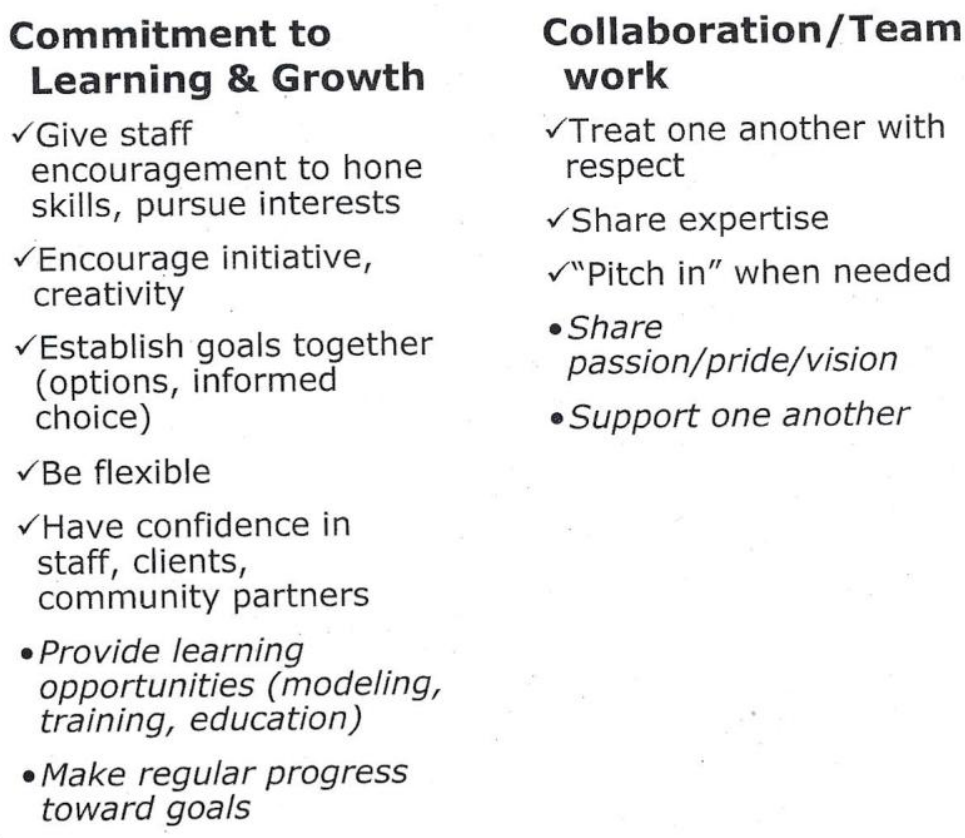

Commitment to Learning \& Growth

$\checkmark$ Give staff encouragement to hone skills, pursue interests

$\checkmark$ Encourage initiative, creativity

$\checkmark$ Establish goals together (options, informed choice)

$\checkmark$ Be flexible

$\checkmark$ Have confidence in staff, clients, community partners

- Provide learning opportunities (modeling, training, education)

- Make regular progress toward goals

Collaboration/Team work

$\checkmark$ Treat one another with respect

$\checkmark$ Share expertise

$\checkmark$ "Pitch in" when needed

- Share passion/pride/vision

- Support one another 
Appendix B - Informed Consent 


\section{Informed Consent for the}

\section{Strengths in Action Project}

\section{DSHS/Division of Vocational Rehabilitation}

You are invited to participate in a research study conducted by Barbara Whitbeck from Portland State University's School of Social Work. This study is being conducted in partial fulfillment of the requirements of the researcher's doctoral degree, under the supervision of Maria Talbott, Ph.D. The researcher hopes to learn about the benefits of, and the barriers to, implementing a project such as Strengths in Action in DVR field offices, and to use the research findings to develop a model that can be used in DVR offices throughout Washington. You are being asked to participate in this study because you are working in one of the DVR offices selected to participate in the Strengths in Action project. Your office was selected by the researcher and the DVR management team because of its unique strengths and its particular challenges; two other DVR field offices will be participating in this study.

If you decide to participate in the Strengths in Action project, you will be asked to work together, in your office, with your supervisor and coworkers to select one short-term goal for your office that can be accomplished in approximately four months; to define the steps necessary to reach that goal; to determine who will be responsible for accomplishing each step; to decide how to measure progress toward the goal; and to develop ways for staff to communicate/collaborate throughout the project. (Where, when, how often, and in what combinations staff will meet will be determined by staff in each office.) On an individual level, you will be asked to commit to, and follow through with, accomplishing one or more tasks in your office or in your office's community that will help your office reach its goal.

During Strengths in Action meetings, and when reviewing related staff communications, the researcher will be taking notes on staff discussions, decisions, and interactions. No statement you make outside of such meetings or related communications will be written down unless the researcher requests, and is granted, your permission to do so. At the end of the project, you will be asked to complete a brief, anonymous, online survey including questions about your experiences with, and impressions of, the Strengths in Action project. You may also be asked by the researcher to participate in a 30-60 minute audio-taped interview about the project.

While participating in the Strengths in Action project, which will involve a variety of group meetings and group activities, it is possible that you may experience discomfort during group interactions, worry about possible repercussions from having your work observed or from what you say about your work, or feel that your participation in the project is keeping you from other work-related duties. If you have such feelings, you have the right to contact your supervisor and ask to be excused from the meeting or activity. You may not receive any direct benefit from taking part in this study, but the study may lead to increased knowledge that may help you, your office, other DVR offices, and DVR customers; improved relationships within your office, or between your office and community partners; and improved services for DVR customers. 
All information that is obtained in connection with this study, and that can be linked to you or identify you, will be kept confidential. Neither you, nor your office, will be named in any reporting of the survey results. For the purposes of this study, the researcher will assign you a pseudonym. Any statements you make or comments pertaining to you in notes from staff meetings or transcripts from interviews will use the pseudonym rather than your name and any identifying characteristics will be omitted. The link between your name and the pseudonym will be stored by the researcher in a password-protected file that only the researcher can access. Field notes and interview data will be stored in a locked cabinet in a secure office, which can only be accessed by the researcher. The data (whether in written or taped form) will be destroyed once the study, and the publication processes associated with it, are complete.

Your participation in the Strengths in Action project is voluntary. You do not have to take part in this study; if you choose to not participate, it will not affect your employment with the Division of Vocational Rehabilitation (DVR). You may also withdraw from this project at any time without affecting your employment with DVR.

If you have concerns or any problems about your participation in this study or your rights as a research subject, please contact the Human Subjects Research Review Committee, Office of Research and Sponsored Projects, 111 Cramer Hall, Portland State University, (503) 725-4288. If you have questions about the study itself, contac t Barbara Whitbeck at (360) 915-3729.

Your signature indicates that you have read and understand the above information and agree to take part in this study. Please understand that you may withdraw your consent at any time without penalty, and that, by signing, you are not waiving any legal claims, rights, or remedies. The researcher will provide you with a copy of this form for your own records. 
Appendix C - Interview Questions 


\section{Interview Questions \\ (Strengths in Action Study)}

Employment History Questions:

How long have you worked at DVR?

How long have you worked in your current office?

What different positions have you held at DVR?

Overview of Strengths in Action Project:

What do you think went well during the Strengths in Action project?

What do you think were the challenges of the Strengths in Action project?

What changes have you noted in the office during the Strengths in Action project?

Probe (if change occurred): Do you think this change will be lasting?

Learning from the Strengths in Action Project:

On a personal level, what did you learn during the Strengths in Action project?

What did your office learn during the Strengths in Action project?

What did you, or your office, learn during the Strengths in Action project that will benefit DVR customers?

Probes (for the 3 questions above, if learning occurred): Will you/your office be able to use this learning in your work setting? In what ways?

Building Relationships during the Strengths in Action Project:

How do you think the Strengths in Action project affected working relationships within your office?

How do you think the Strengths in Action project affected your office's working relationships with community partners?

Other:

Is there anything else about the Strengths in Action project that you would like to share? 
Appendix D - Online Survey 


\section{Strengths in Action}

\section{1. matrix questions}

Weicome to the STRENGTHS IN ACTION SUrvey!

1. The questions below are about your office's participation in the Strengths in Action project. Please indicate the degree to which you agree with the following statements.

The supervisor and staff in my office agreed to participate in the project

The supervisor and staff in my office worked together to select ane short-term goal for the project

The supervisor and staff in my office each agreed to do one or more things to help the oflice reach the goal

The supervisor and stoff in my office each did one ar more things to help the office reach the goal

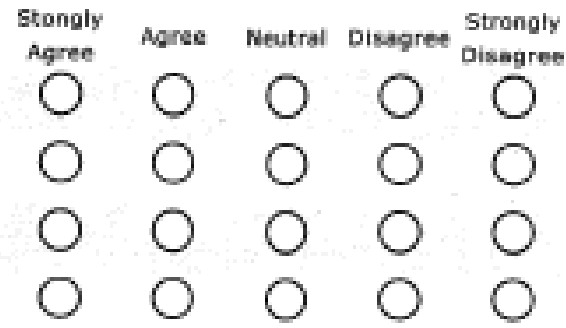

2. The questions below are about what you and your office learned during the Strengths in Action project. Please indicate the degree to which you agree with the following statements.

I took on one or more tasks that 1 had not done at work before

I learned something about my own abetties snd/or interests

I learned something about the abilities and/or interests of one or more of my co-workers

I learned ane or more wars my co-morkers and I ean tetter heip DVR customers

I learned one or more ways individuals or orgonizations outside DVR (community partners, employers, ete.) can help DVh custamers

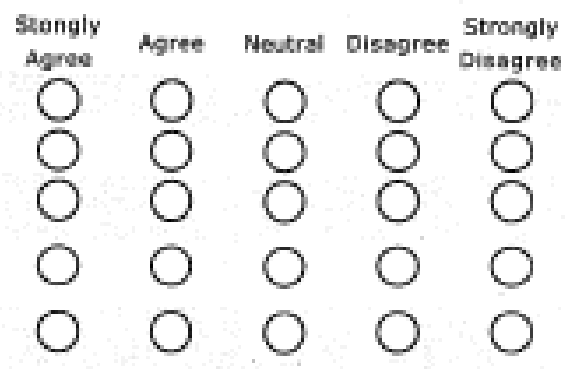

\section{The questions below are about the ways you communicated and} collaborated with your supervisor, co-workers and others during the Strengths in Action project. Please indicate the degree to which you agree with the following statements.

Ner supervisor, co-morkers and I shared new knowiedge and insights
Ny supervisor, co-morkars and I workad together on one sr more parts
of the project
My supervisor, co-morkers and I kept one another intormed of
progress made on the project.
My supervisor, co-workers and I became a stronger team
My supervisor, co-workers and I developed a stronger working
relationship with others (DVk customers, community partners,




\section{Strengths in Action}

\section{Default Section}

The questions below are absut vaur averall reaction to the Strengths in Action project.

4. For you, what were the benefits of the Strengths in Action project?

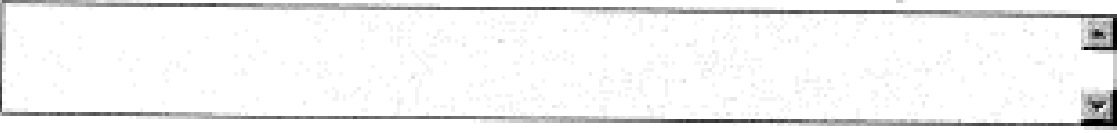

5. For your office as a whole, what were the benefits of the Strengths in Action project?

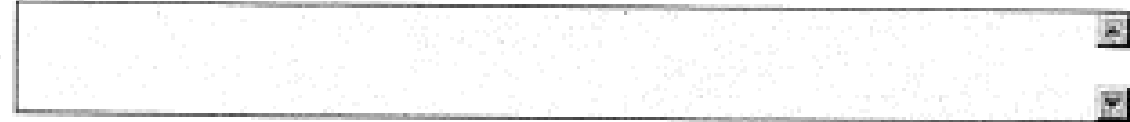

6. For DVR customers, what were the benefits of the Strengths in Action project?

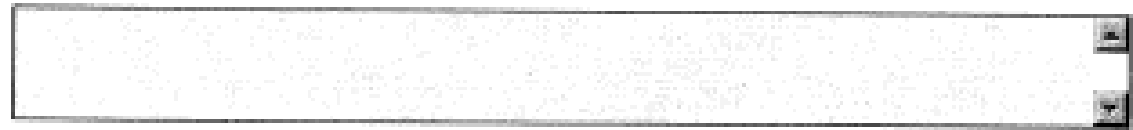

7. How do you think the Strengths in Action project could be improved?

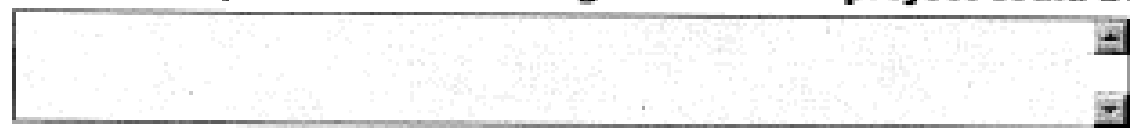




\section{Strengths in Action}

\section{3. thank you}

Thanks for your partidontion in this survey. Press "Done" below to complete the survey, Make sure you are campletely done with the survey before you press "Done."

After the survey closes, you may see a message thot says abmething like "Trying to clese Window," Don't worry about this. Your surver is safely closed. 
Appendix E - Description of Strengths in Action Study 


\section{STRENGTHS IN ACTION}

\section{What is the STRENGTHS IN ACTION project?}

This project is designed to build the strongest possible teams within DVR offices and units, as making the most of our staff's talents is the best way for DVR to serve customers and communities well. The project is based on learning organization theory, which stresses that staff should be encouraged to:

- Recognize, and develop, their individual strengths/interests

- Use those strengths/interests in the workplace

- Collaborate with coworkers and community partners

- Constantly reflect, as a group, on whether they are doing what is needed to serve their customers well.

The steps outlined above, if practiced consistently, will result in teams that serve their customers efficiently and effectively, and can adapt to inevitable changes in the work environment.

What is the connection between STRENGTHS IN ACTION and two other recent DVR trainings - Strengths Training, and Motivational Interviewing?

In both Strengths Training and Motivational Interviewing, staff members were asked to look closely at their particular interests, talents, and skills. The STRENGTHS IN ACTION project will ask staff members to put their individual strengths to "best use" while working together with their coworkers to reach a common goal.

Who will be involved in the STRENGTHS IN ACTION project?

The pilot project will involve 3 DVR offices or units: [Names of offices/units redacted].

What are staff participating in the STRENGTHS IN ACTION project expected to do?

- Staff will be given information from the Strengths Training (specific to themselves and their office/unit) and asked to review and reflect on it before beginning the pilot process.

- The VRS and office/unit staff will meet and define one short-term goal that is important to them.

- The VRS and office/unit staff will discuss and decide how to accomplish the short-term goal. This will include who will do what (taking individual strengths into consideration), a timeline, how the office/unit will communicate during the process, and expected outcomes.

- Staff will take steps to address the goal, and periodically check in with one another to report on progress and make adjustments necessary to move forward. 
- At the end of the pilot process, staff will be asked to evaluate the experience.

How long will this process take?

About two to four months. (Staff in each office/unit will set their own goal, and establish their own timeline.)

Who, outside of the VRS and unit staff is involved in the process?

[Name redacted], DVR Program Manager, will assist in any way that is helpful to the supervisor and staff during the pilot process.

Barbara Whitbeck, DSHS-RDA employee, will evaluate the STRENGTHS IN ACTION pilot process as her dissertation project. (She's a Ph.D. candidate at Portland State University School of Social Work.) 
Appendix F - First Strengths in Action Agenda 


\section{STRENGTHS IN ACTION Agenda}

[Name of office and meeting date redacted]

1

\section{Today's task is to select ONE goal that all staff can agree:}

- Will benefit office staff

- Will benefit DVR clients

- Will include some role for ALL staff in the office

- Will take 2-4 months to accomplish (this timeline is approximate)

- Will be measurable (there are ways to know if the goal has been reached)

\section{Follow these steps:}

- List possible goals*

- Discuss the merits of the goals listed

- Decide upon a single goal

- Decide when staff with meet again to develop a plan to reach the goal

\section{*Possible Goals for DVR Field Offices:}

- Outreach - new referrals, broaden population of eligible customers

- Building relationships with local employers (focus on certain sectors)

- Building relationships with community partners (focus on certain individuals, organizations)

- Transition

- Developing/improving onsite orientations or trainings

- Improving staff processes - contacting/scheduling customers, staff communications, etc.

- Improving particular skills - motivational interviewing, writing plans, etc. 
Appendix G - Response Glossary 


\section{Strengths in Action (SIA) Online Survey - Response Glossary}

\begin{tabular}{|c|c|}
\hline \multicolumn{2}{|c|}{$\begin{array}{l}\text { LEARNING ORGANIZATION DISCIPLINES } \\
\text { Note: Relates to Research Question 1: } \\
\text { To what extent was a modified learning organization model implemented? }\end{array}$} \\
\hline Response Category & Description \\
\hline DP - Personal Mastery & $\begin{array}{l}\text { An individual learned something of value - a new skill, new } \\
\text { experience, or new behavior. Moved beyond their comfort } \\
\text { zone. "I learned something"; "I didn't learn anything." }\end{array}$ \\
\hline DM - Mental Models & $\begin{array}{l}\text { An individual examined, or changed, their point of view about } \\
\text { something (work-related, or otherwise). }\end{array}$ \\
\hline DV - Shared Vision & $\begin{array}{l}\text { Staff worked together to decide on a goal. Staff reached } \\
\text { consensus on a goal. Staff had a common investment in the } \\
\text { goal. Coworkers developed new working relationships with } \\
\text { one another. }\end{array}$ \\
\hline DT - Team Learning & $\begin{array}{l}\text { Staff worked together to reach a particular goal. Staff agreed on } \\
\text { the steps necessary to reach the goal. Staff agreed on roles for } \\
\text { each staff member. Staff learned from each other during the } \\
\text { SIA Project. Staff learned from community partners during the } \\
\text { SIA Project. "We learned something"; "We didn't learn } \\
\text { anything." }\end{array}$ \\
\hline DS - Systems Thinking & $\begin{array}{l}\text { Staff shared information and ideas during SIA. Staff agreed } \\
\text { upon and/or made necessary adjustments during SIA (iterative } \\
\text { process). Staff took individual strengths into account while } \\
\text { progressing toward the goal. Staff reported value in learning } \\
\text { organization experiences. }\end{array}$ \\
\hline
\end{tabular}

\begin{tabular}{|c|c|}
\hline \\
\hline \multicolumn{2}{|c|}{$\begin{array}{l}\text { FACILITATING/IMPEDING FACTORS } \\
\text { Note: Relates to Research Questions } 2 \text { and 3: } \\
\text { What factors facilitated the implementation of a modified learning organization model? } \\
\text { What factors impeded the implementation of a modified learning organization model? }\end{array}$} \\
\hline \multicolumn{2}{|c|}{\begin{tabular}{l|l} 
FT - Time & $\begin{array}{l}\text { SIA created time and space to work on a project of value. SIA } \\
\text { should have lasted longer. SIA took time away from more } \\
\text { important work tasks. Hard to find time to do SIA Project- }\end{array}$
\end{tabular}} \\
\hline FS - Supervisor Support & $\begin{array}{l}\text { DVR Supervisor supported - or didn't support - the SIA Project } \\
\text { concept. Supervisor supported - or didn't support - the SIA } \\
\text { Project in practical ways. Supervisor was invested, or not } \\
\text { invested, in project. }\end{array}$ \\
\hline $\begin{array}{l}\text { FH -Headquarters Staff } \\
\text { Support }\end{array}$ & $\begin{array}{l}\text { DVR Headquarters Staff (other than project facilitator) } \\
\text { supported - or didn't support - the SIA Project concept. } \\
\text { Headquarters Staff supported - or didn't support - the SIA } \\
\text { Project in practical ways. Headquarters staff was invested, or }\end{array}$ \\
\hline
\end{tabular}




\begin{tabular}{|l|l|}
\hline Response Category & not invested, in project. \\
\hline $\begin{array}{l}\text { FD - Staff Support } \\
\text { (Teamwork) }\end{array}$ & $\begin{array}{l}\text { DVR Staff supported - or didn't support - the SIA Project } \\
\text { concept. Staff supported - or didn't support - the SIA Project, } \\
\text { in practical ways. (Support, or lack of, being directed at the } \\
\text { project as a whole, or at certain coworkers.) Ownership of, and } \\
\text { investment in, project occurred or didn't occur. Comments } \\
\text { about teamwork, or lack of teamwork. }\end{array}$ \\
\hline FF- Facilitator Support & $\begin{array}{l}\text { DVR headquarters staff facilitator supported - or didn't support } \\
\text { - the SIA Project concept. Facilitator supported - or didn't } \\
\text { support - the SIA Project in practical ways. Facilitator was } \\
\text { invested, or not invested, in project. }\end{array}$ \\
\hline $\begin{array}{l}\text { FC -Community } \\
\text { Partner Support }\end{array}$ & $\begin{array}{l}\text { Interactions with individuals or agencies outside of DVR that } \\
\text { moved the SIA Project forward (i.e., responsiveness, } \\
\text { cooperation, offers of assistance) or impeded the SIA Project } \\
\text { (i.e., inability to contact community partners, lack of response, } \\
\text { refusal to cooperate). }\end{array}$ \\
\hline $\begin{array}{l}\text { FP - SIA Project } \\
\text { Structure/Process }\end{array}$ & $\begin{array}{l}\text { Positive or negative comments about the structure of the SIA } \\
\text { Project. (Includes comments about agendas, regular meetings, } \\
\text { focused discussions, working in small groups, accountability of } \\
\text { staff. Also, needing more guidance, structure.) }\end{array}$ \\
\hline FA - Agency Structure & $\begin{array}{l}\text { Positive or negative comments about the impact DVR's } \\
\text { organizational structure had on the SIA Project. Comments } \\
\text { about agency focus on individual rather than team efforts } \\
\text { belong here. }\end{array}$ \\
\hline FE - Economy & $\begin{array}{l}\text { Positive or negative impacts the economy had on the SIA } \\
\text { Project. }\end{array}$ \\
\hline FO - Other factors & Other factors that facilitated or impeded the SIA Project. \\
\hline
\end{tabular}

\begin{tabular}{|l|l|}
\hline OFFICE CLIMATE AND CULTURE \\
\hline $\begin{array}{l}\text { Note: Relates to Research Question 4: } \\
\text { What impact did the implementation (or partial implementation) of a modified learning } \\
\text { organization model have on office climate and culture? }\end{array}$ \\
\hline \multicolumn{1}{|c|}{ Response Category } & \multicolumn{1}{c|}{ Description } \\
\hline $\begin{array}{l}\text { CP- Positive Impacts } \\
\text { on Office Climate and } \\
\text { Culture }\end{array}$ & $\begin{array}{l}\text { Improved communication, bonding, understanding, among } \\
\text { coworkers. Improved ability to work together (includes } \\
\text { new/different working relationships formed within an office or } \\
\text { unit). Improved recognition, use of, and valuing of specific } \\
\text { strengths of individual staff members. }\end{array}$ \\
\hline $\begin{array}{l}\text { CN - Negative Impacts } \\
\text { on Office Climate and } \\
\text { Culture }\end{array}$ & $\begin{array}{l}\text { Conflict or tension among staff members. Tasks related to SIA } \\
\text { Project added additional stress/pressure. SIA Project lowered } \\
\text { morale. }\end{array}$ \\
\hline
\end{tabular}




\begin{tabular}{|l|l|}
\hline \multicolumn{1}{|c|}{ PROJECT OUTCOMES } \\
\hline Response Category & \multicolumn{1}{c|}{ Description } \\
\hline PB - DVR Customers & $\begin{array}{l}\text { The SIA Project has, or will be, beneficial to DVR customers. } \\
\text { The SIA Project hasn't, or won't be, beneficial to DVR } \\
\text { customers. }\end{array}$ \\
\hline $\begin{array}{l}\text { PC - Community } \\
\text { Partners }\end{array}$ & $\begin{array}{l}\text { The SIA Project has improved, or will improve, working } \\
\text { relationships with community partners. The SIA Project hasn't } \\
\text { improved, or won't improve, working relationships with } \\
\text { community partners. }\end{array}$ \\
\hline PS - Sustainability & $\begin{array}{l}\text { Gains made during the SIA Project will, or won't, be } \\
\text { sustainable. }\end{array}$ \\
\hline PO - Other outcomes & Other positive or negative outcomes of the SIA Project. \\
\hline
\end{tabular}

\begin{tabular}{|l|l|}
\hline \begin{tabular}{l} 
OTHER \\
\hline \multicolumn{1}{|c|}{ Response Category }
\end{tabular} & \multicolumn{1}{c|}{ Description } \\
\hline ON - Nothing & $\begin{array}{l}\text { Nothing. Can't think of anything. (Negative if Q4-6, Positive if } \\
\text { Q7.) }\end{array}$ \\
\hline OD - Don't Know & Don't know. Don't have an answer. Unsure. \\
\hline O- Other & Miscellaneous comments that don't fit elsewhere. \\
\hline
\end{tabular}

\section{NOTES:}

Because the codes are designed to refer back to the first four research questions, certain comments may fit in a variety of places. For example:

"Our staff benefited from the continued meetings within our office - even if these meetings were very brief. We were able to communicate with each other new ideas and others that have not worked." FP, DT, DS, CP

"I got out of my comfort zone, and found a new agency to connect with in the community." DP, PC

Some codes will be used infrequently when coding the brief online survey responses; they are included because the same domains will be used when coding the more extensive interviews. 
Appendix H - ANOVA Results 
ANOVA Results*

\begin{tabular}{|c|c|c|c|c|c|c|}
\hline Question & & $\begin{array}{c}\text { Sum of } \\
\text { Squares }\end{array}$ & df & $\begin{array}{c}\text { Mean } \\
\text { Square }\end{array}$ & $\mathbf{F}$ & Sig. \\
\hline FIPartic & $\begin{array}{c}\text { Between Groups } \\
\text { Within Groups } \\
\text { Total }\end{array}$ & $\begin{array}{r}1.021 \\
43.952 \\
44.973 \\
\end{array}$ & $\begin{array}{r}2 \\
34 \\
36 \\
\end{array}$ & $\begin{array}{r}.511 \\
1.293\end{array}$ & .395 & .677 \\
\hline F2Goal & $\begin{array}{c}\text { Between Groups } \\
\text { Within Groups } \\
\text { Total }\end{array}$ & \begin{tabular}{r|}
1.717 \\
32.391 \\
34.108 \\
\end{tabular} & $\begin{array}{r}2 \\
34 \\
36 \\
\end{array}$ & $\begin{array}{l}.858 \\
.953\end{array}$ & .901 & .416 \\
\hline F3Agreed & $\begin{array}{c}\text { Between Groups } \\
\text { Within Groups } \\
\text { Total }\end{array}$ & \begin{tabular}{r|}
2.511 \\
41.759 \\
42.270 \\
\end{tabular} & $\begin{array}{r}2 \\
34 \\
36 \\
\end{array}$ & $\begin{array}{l}1.255 \\
1.228\end{array}$ & 1.022 & .371 \\
\hline F4help & $\begin{array}{c}\text { Between Groups } \\
\text { Within Groups } \\
\text { Total }\end{array}$ & $\begin{array}{r}3.670 \\
44.005 \\
47.676 \\
\end{array}$ & $\begin{array}{r}2 \\
34 \\
36 \\
\end{array}$ & $\begin{array}{l}1.835 \\
1.294\end{array}$ & 1.418 & .256 \\
\hline L1Task & $\begin{array}{c}\text { Between Groups } \\
\text { Within Groups } \\
\text { Total }\end{array}$ & $\begin{array}{r}.046 \\
44.765 \\
44.811 \\
\end{array}$ & $\begin{array}{r}2 \\
34 \\
36\end{array}$ & $\begin{array}{r}.023 \\
1.317\end{array}$ & .017 & .983 \\
\hline L2Own & $\begin{array}{c}\text { Between Groups } \\
\text { Within Groups } \\
\text { Total }\end{array}$ & $\begin{array}{r}3.121 \\
38.122 \\
41.243 \\
\end{array}$ & $\begin{array}{r}2 \\
34 \\
36\end{array}$ & $\begin{array}{l}1.561 \\
1.121\end{array}$ & 1.392 & .262 \\
\hline L3Cowork & $\begin{array}{c}\text { Between Groups } \\
\text { Within Groups } \\
\text { Total }\end{array}$ & $\begin{array}{r}.621 \\
40.298 \\
40.919 \\
\end{array}$ & $\begin{array}{r}2 \\
34 \\
36 \\
\end{array}$ & $\begin{array}{r}.311 \\
1.185\end{array}$ & .262 & .771 \\
\hline L4Help & $\begin{array}{c}\text { Between Groups } \\
\text { Within Groups } \\
\text { Total }\end{array}$ & $\begin{array}{r}3.143 \\
35.559 \\
38.703 \\
\end{array}$ & $\begin{array}{r}2 \\
34 \\
36 \\
\end{array}$ & $\begin{array}{l}1.572 \\
1.046\end{array}$ & 1.503 & .237 \\
\hline L5Outside & $\begin{array}{c}\text { Between Groups } \\
\text { Within Groups } \\
\text { Total } \\
\end{array}$ & $\begin{array}{r}1.022 \\
20.005 \\
21.027 \\
\end{array}$ & $\begin{array}{r}2 \\
34 \\
36 \\
\end{array}$ & $\begin{array}{l}.511 \\
.588\end{array}$ & .868 & .429 \\
\hline C1Shared & $\begin{array}{c}\text { Between Groups } \\
\text { Within Groups } \\
\text { Total }\end{array}$ & $\begin{array}{r}.939 \\
33.169 \\
34.108\end{array}$ & $\begin{array}{r}2 \\
34 \\
36\end{array}$ & $\begin{array}{l}.469 \\
.976\end{array}$ & .481 & .622 \\
\hline C2Together & $\begin{array}{c}\text { Between Groups } \\
\text { Within Groups } \\
\text { Total } \\
\end{array}$ & $\begin{array}{r}4.619 \\
34.381 \\
39.000 \\
\end{array}$ & $\begin{array}{r}2 \\
33 \\
35 \\
\end{array}$ & $\begin{array}{l}2.310 \\
1.042\end{array}$ & 2.217 & .125 \\
\hline C3Inform & $\begin{array}{c}\text { Between Groups } \\
\text { Within Groups } \\
\text { Total }\end{array}$ & $\begin{array}{r}4.600 \\
27.292 \\
31.892\end{array}$ & $\begin{array}{r}2 \\
34 \\
36\end{array}$ & $\begin{array}{r}2.300 \\
.803\end{array}$ & 2.865 & .071 \\
\hline C4Team & $\begin{array}{c}\text { Between Groups } \\
\text { Within Groups } \\
\text { Total }\end{array}$ & $\begin{array}{r}.615 \\
30.088 \\
30.703\end{array}$ & $\begin{array}{r}2 \\
34 \\
36\end{array}$ & $\begin{array}{l}.307 \\
.885\end{array}$ & .347 & .709 \\
\hline C5Develop & $\begin{array}{c}\text { Between Groups } \\
\text { Within Groups } \\
\text { Total }\end{array}$ & $\begin{array}{r}2.342 \\
34.577 \\
36.919\end{array}$ & $\begin{array}{r}2 \\
34 \\
36\end{array}$ & $\begin{array}{l}1.171 \\
1.017\end{array}$ & 1.151 & .328 \\
\hline
\end{tabular}

\footnotetext{
* Refer to Appendix I for wording of questions F1-C5.
} 
Appendix I - Kruskal-Wallis Results 


\section{Kruskal-Wallis Results}

\begin{tabular}{|l|r|r|r|}
\hline \multicolumn{1}{|c|}{ Question } & Chi-Square & df & Asymp. Sig. \\
\hline FIPartic & 1.265 & 2 & .531 \\
\hline F2Goal & 1.335 & 2 & .513 \\
\hline F3Agreed & 1.439 & 2 & .487 \\
\hline F4help & 2.888 & 2 & .236 \\
\hline L1Task & .515 & 2 & .773 \\
\hline L2Own & 2.404 & 2 & .301 \\
\hline L3Cowork & .478 & 2 & .788 \\
\hline L4Help & 2.849 & 2 & .241 \\
\hline L5Outside & 2.098 & 2 & .350 \\
\hline C1Shared & .863 & 2 & .650 \\
\hline C2Together & 4.083 & 2 & .130 \\
\hline C3Inform & 4.118 & 2 & .128 \\
\hline C4Team & .457 & 2 & .796 \\
\hline C5Develop & 2.200 & 2 & .333 \\
\hline
\end{tabular}

\section{Questions:}

FI: The supervisor and staff in my office agreed to participate in the project

F2: The supervisor and staff in my office worked together to select one short-term goal for the project

F3: The supervisor and staff in my office each agreed to do one or more things to help the office reach the goal

F4: The supervisor and staff in my office each did one or more things to help the office reach the goal

L1: I took on one or more tasks that I had not done at work before

L2: I learned something about my own abilities and/or interests

L3: I learned something about the abilities and/or interests of one or more of my co-workers

L4: I learned one or more ways my co-workers and I can better help DVR customers

L5: I learned one or more ways individuals and organizations outside DVR (community partners, employers, etc.) can help DVR customers

C1: My supervisor, co-workers and I shared new knowledge and insights

C2: My supervisor, co-workers and I worked together on one or more parts of the project

C3: My supervisor, co-workers and I kept one another informed of progress made on the project

C4: My supervisor, co-workers and I became a stronger team

C5: My supervisor, co-workers and I developed a stronger working relationship with others (DVR customers, community partners, employers, etc.) 
Appendix J — Online Survey Closed-ended Questions: Means, Standard Deviations, and Percentages of Positive Responses 


\section{Online Survey Closed-ended Questions:}

\section{Means, Standard Deviations, and Percentage of Positive Responses}

Fidelity Questions:

FI: The supervisor and staff in my office agreed to participate in the project

F2: The supervisor and staff in my office worked together to select one short-term goal for the project

F3: The supervisor and staff in my office each agreed to do one or more things to help the office reach the goal

F4: The supervisor and staff in my office each did one or more things to help the office reach the goal

\begin{tabular}{|l|c|r|r|r|r|}
\hline Question & Office & N & Mean & $\begin{array}{c}\text { Standard } \\
\text { Deviation }\end{array}$ & $\begin{array}{c}\text { \% Strongly } \\
\text { Agree or } \\
\text { Agree }\end{array}$ \\
\hline F1Partic & Office A & 13 & 3.92 & .862 & $62 \%$ \\
& Office B & 14 & 3.93 & 1.439 & $71 \%$ \\
& Office C & 10 & 4.30 & .949 & $90 \%$ \\
& Total & 37 & 4.03 & 1.118 & $73 \%$ \\
\hline F2Goal & Office A & 13 & 4.62 & .506 & $100 \%$ \\
& Office B & 14 & 4.14 & 1.099 & $86 \%$ \\
& Office C & 10 & 4.20 & 1.229 & $80 \%$ \\
& Total & 37 & 4.32 & .973 & $89 \%$ \\
\hline F3Agreed & Office A & 13 & 4.54 & .660 & $93 \%$ \\
& Office B & 14 & 3.93 & 1.328 & $79 \%$ \\
& Office C & 10 & 4.20 & 1.229 & $80 \%$ \\
& Total & 37 & 4.22 & 1.109 & $84 \%$ \\
\hline F4help & Office A & 13 & 4.62 & .650 & $92 \%$ \\
& Office B & 14 & 3.93 & 1.207 & $79 \%$ \\
& Office C & 10 & 4.00 & 1.491 & $70 \%$ \\
& Total & 37 & 4.19 & 1.151 & $81 \%$ \\
\hline
\end{tabular}




\section{Learning Questions:}

L1: I took on one or more tasks that I had not done at work before

L2: I learned something about my own abilities and/or interests

L3: I learned something about the abilities and/or interests of one or more of my coworkers

L4: I learned one or more ways my co-workers and I can better help DVR customers

L5: I learned one or more ways individuals and organizations outside DVR (community partners, employers, etc.) can help DVR customers

\begin{tabular}{|l|c|r|r|r|r|}
\hline Question & Office & $\mathbf{N}$ & Mean & $\begin{array}{c}\text { Standard } \\
\text { Deviation }\end{array}$ & $\begin{array}{c}\text { \% Strongly } \\
\text { Agree or } \\
\text { Agree }\end{array}$ \\
\hline L1Task & Office A & 13 & 3.77 & 1.363 & $69 \%$ \\
& Office B & 14 & 3.79 & 1.122 & $78 \%$ \\
& Office C & 10 & 3.70 & .823 & $70 \%$ \\
& Total & 37 & 3.76 & 1.116 & $73 \%$ \\
\hline L2Own & Office A & 13 & 3.77 & 1.116 & $53 \%$ \\
& Office B & 14 & 3.14 & 1.027 & $36 \%$ \\
& Office C & 10 & 3.70 & .949 & $60 \%$ \\
& Total & 37 & 3.51 & 1.070 & $49 \%$ \\
\hline L3Cowork & Office A & 13 & 3.69 & .947 & $54 \%$ \\
& Office B & 14 & 3.43 & 1.158 & $57 \%$ \\
& Office C & 10 & 3.70 & 1.160 & $70 \%$ \\
& Total & 37 & 3.59 & 1.066 & $60 \%$ \\
\hline L4Help & Office A & 13 & 3.46 & 1.050 & $38 \%$ \\
& Office B & 14 & 3.43 & 1.089 & $50 \%$ \\
& Office C & 10 & 4.10 & .876 & $70 \%$ \\
& Total & 37 & 3.62 & 1.037 & $51 \%$ \\
\hline L5Outside & Office A & 13 & 3.62 & .650 & $54 \%$ \\
& Office B & 14 & 3.93 & .829 & $78 \%$ \\
& Office C & 10 & 4.00 & .816 & $70 \%$ \\
& Total & 37 & 3.84 & .764 & $68 \%$ \\
\hline
\end{tabular}




\section{Communication and Collaboration Questions:}

C1: My supervisor, co-workers and I shared new knowledge and insights

C2: My supervisor, co-workers and I worked together on one or more parts of the project

C3: My supervisor, co-workers and I kept one another informed of progress made on the project

C4: My supervisor, co-workers and I became a stronger team

C5: My supervisor, co-workers and I developed a stronger working relationship with others (DVR customers, community partners, employers, etc.)

\begin{tabular}{|l|c|r|r|r|r|}
\hline Question & Office & N & Mean & $\begin{array}{c}\text { Standard } \\
\text { Deviation }\end{array}$ & $\begin{array}{c}\text { \% Strongly } \\
\text { Agree or } \\
\text { Agree }\end{array}$ \\
\hline C1Shared & Office A & 13 & 3.69 & .751 & $53 \%$ \\
& Office B & 14 & 3.50 & 1.225 & $57 \%$ \\
& Office C & 10 & 3.90 & .876 & $80 \%$ \\
& Total & 37 & 3.68 & .973 & $62 \%$ \\
\hline C2Together & Office A & 12 & 4.33 & .492 & $99 \%$ \\
& Office B & 14 & 3.64 & 1.216 & $71 \%$ \\
& Office C & 10 & 3.50 & 1.179 & $70 \%$ \\
& Total & 36 & 3.83 & 1.056 & $80 \%$ \\
\hline C3Inform & Office A & 13 & 4.15 & .555 & $92 \%$ \\
& Office B & 14 & 3.50 & 1.160 & $64 \%$ \\
& Office C & 10 & 4.30 & .823 & $80 \%$ \\
& Total & 37 & 3.95 & .941 & $78 \%$ \\
\hline C4Team & Office A & 13 & 3.46 & .660 & $39 \%$ \\
& Office B & 14 & 3.21 & 1.188 & $43 \%$ \\
& Office C & 10 & 3.50 & .850 & $50 \%$ \\
& Total & 37 & 3.38 & .924 & $43 \%$ \\
\hline C5Develop & Office A & 13 & 3.38 & .768 & $39 \%$ \\
& Office B & 14 & 3.50 & 1.225 & $57 \%$ \\
& Office C & 10 & 4.00 & .943 & $60 \%$ \\
& Total & 37 & 3.59 & 1.013 & $52 \%$ \\
\hline
\end{tabular}

